Portland State University

PDXScholar

Spring 5-9-2014

\title{
Guiding the Work of Professional Learning \\ Communities: Perspectives for School Leaders
}

Daniel Paul Draper

Portland State University

Follow this and additional works at: https://pdxscholar.library.pdx.edu/open_access_etds

Part of the Educational Administration and Supervision Commons, and the Educational Leadership Commons

Let us know how access to this document benefits you.

\section{Recommended Citation}

Draper, Daniel Paul, "Guiding the Work of Professional Learning Communities: Perspectives for School Leaders" (2014). Dissertations and Theses. Paper 1823.

https://doi.org/10.15760/etd.1822

This Dissertation is brought to you for free and open access. It has been accepted for inclusion in Dissertations and Theses by an authorized administrator of PDXScholar. Please contact us if we can make this document more accessible: pdxscholar@pdx.edu. 
Guiding the Work of Professional Learning Communities:

Perspectives for School Leaders

\author{
by \\ Daniel Paul Draper
}

A dissertation submitted in partial fulfillment of the requirements for the degree of

\title{
Doctor of Education
}

in

Educational Leadership: Administration

Dissertation Committee:

Tom Chenoweth, Chair

Swapna Mukhopadhyay

Dannelle Stevens

Tucker Childs

Portland State University

2014 
(C) 2014 Daniel Paul Draper 


\begin{abstract}
Professional Learning Communities (PLCs) are groups of educators committed to working collaboratively in ongoing processes of collective inquiry and action research to achieve better results for the students they serve. PLCs operate under the assumption that the key to improved learning for students is continuous job-embedded learning for educators (DuFour, DuFour, Eaker, \& Many, 2006). Researchers and practitioners agree that PLCs are critical to the overall success of schools. The problem is that implementing PLCs with fidelity to an inquiry process is a real challenge. Most school districts do not have a systematic or comprehensive approach to guide their PLC process. School leaders are in need of quality tools and resources to assist them in implementing PLCs.

As a possible solution to this problem, a design team of four Estacada School District principals and one vice principal was convened to create, field-test and refine a handbook for PLC leadership. The handbook was field-tested in four schools and evaluated to determine its usefulness. The study's primary research questions were: (a) Is the PLC handbook a useful resource for school leaders? and (b) What are the handbook's strengths and weaknesses? Secondary research questions focused on specific topics and sections of the handbook: (a) How do school leaders organize and support a PLC framework? (b) How can PLCs support school change initiatives? (c) How can PLCs gather and analyze student data? (d) How can PLCs plan for future action? and (e) How can PLCs troubleshoot challenges?
\end{abstract}


The design team relied on a problem-based learning approach (Bridges \& Hallinger, 1995) and the use of a research and development process (Borg \& Gall, 1989) to design an educational product ready for operational use in their schools. The design team met weekly for regularly scheduled meetings. They used the Critical Friends Consultancy Protocol (Harmony Education Center, 2013) as a systematic way to problem solve and collect qualitative data. The data collected from these sessions were transcribed, coded for themes, and analyzed. Other data sources that were used included the review of institutional documentation, structured interviews with teacher leaders, and survey results. The design team then refined its PLC handbook through the first seven steps of the research and development process: (a) Research and information collecting; (b) Planning objectives, learning activities, and small scale testing; (c) Developing a preliminary form of the product; (d) Preliminary field-testing; (e) Main product revision; (f) Main field-testing; and (g) Operational product revision.

The design team determined that the handbook was in fact a useful resource for school leaders, and it helped move PLC work forward in each of the four schools. The team found that the handbook had a number of strengths, including the clarification of key terminology and the establishment of a common language for PLCs. Another noted strength was that the activities included in the handbook were user-friendly. A noted opportunity was that the field-tested handbook did not create viable ways to involve parents, families, and community members in PLC work alongside educators. This opportunity is being addressed by the design team in future handbook revisions. 
The handbook helped school leaders organize and support a PLC framework. The design team confirmed that the handbook assisted PLCs in completing the work required of major school change initiatives, including Differentiated Instruction/Sheltered Instruction, Response to Intervention/Positive Behavioral Intervention Support, Common Core State Standards, and Proficiency-Based Learning. The design team also found the PLC handbook to be useful as an orientation tool for new staff members, as well as a valuable review tool for PLC veterans, particularly regarding how to collect and analyze student assessment data. The handbook also helped PLCs plan future action relative to providing intervention and enrichment opportunities for students. Finally, the handbook provided tools to help educators troubleshoot challenges that surfaced during their PLC work.

The design team will continue to refine its handbook and provide support for the Estacada School District and community as mutually-beneficial PLC-related activities, grants, and projects are pursued. The optimal next step for future use of the handbook would be for several schools and districts throughout Oregon, particularly from small, rural areas, to pilot the handbook. The piloting schools and districts could then share the roadblocks and success stories pertinent to their use of the handbook, which would in turn support the design team in making a quality final product revision. 


\section{Dedication}

This study is dedicated to all of the principals and educators working hard in our schools-may you always find school to be a place that stimulates creativity and problem solving as we all work to make positive contributions to society. 


\section{Acknowledgments}

I am thankful for the many suggestions and support I received throughout this journey. First, I would like to thank my family-Melanie, Mack, and Greta Draper for their patience and understanding as I spent many hours away from them in order to conquer this personal goal. I also want to acknowledge the contributions of the administrators, teachers, and staff of the Estacada School District for participating in this study, and providing helpful insights, feedback, and data. I would also like to thank my doctoral cohort group. We helped push each other to the finish line, and I know we all feel a strong sense of accomplishment. I would like to thank Swapna Mukhopadhyay, Dannelle Stevens, and Tucker Childs for joining my committee, and for offering words of inspiration along the way. Finally, I would like to thank Tom Chenoweth for helping me navigate through this process at Portland State University. His commitment to me and his encouragement of me to complete this journey never wavered. 
Table of Contents

Page

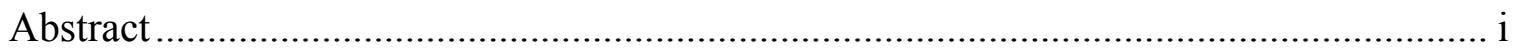

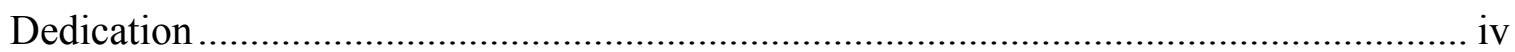

Acknowledgments...........................................................................................

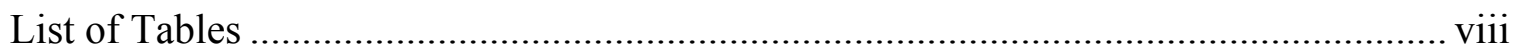

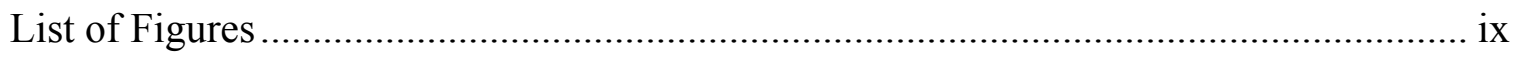

Preface

\section{CHAPTER}

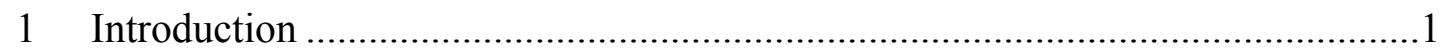

Statement of the Problem...........................................................................

Purpose and Significance of the Study ………………............................10

Research Methodology .......................................................................11

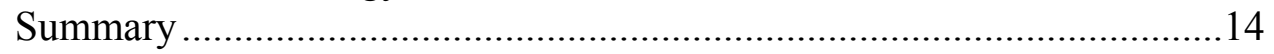

Definition of Key Terms ...................................................................14

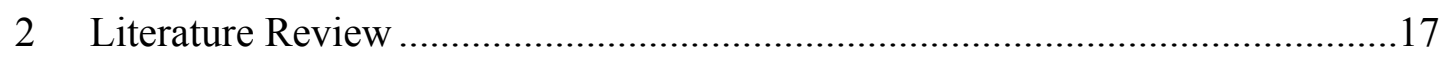

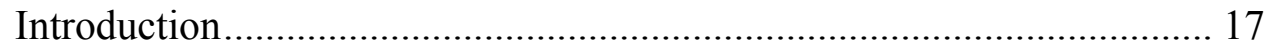

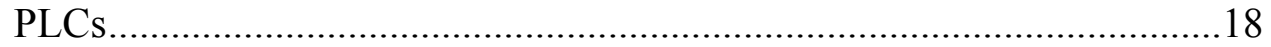

School Change Initiatives .......................................................................

Principal Leadership ………………………………………………....61

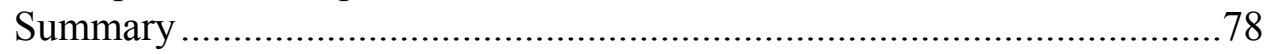

3 Methodology and Research Techniques..........................................................

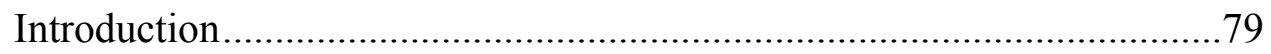

Brief Restatement of the Problem..........................................................81

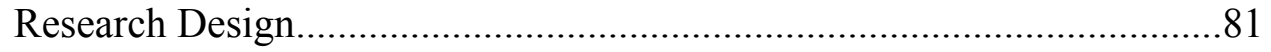

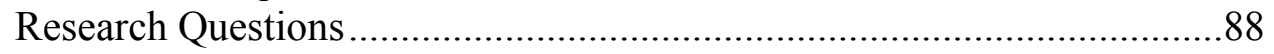




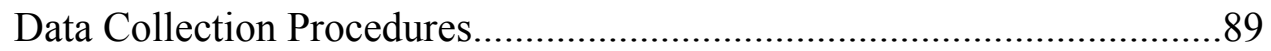

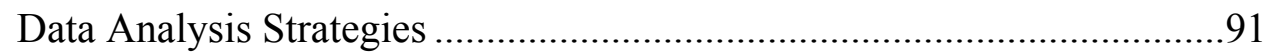

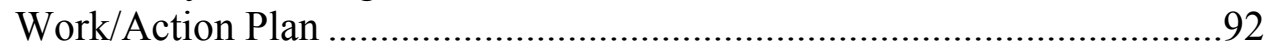

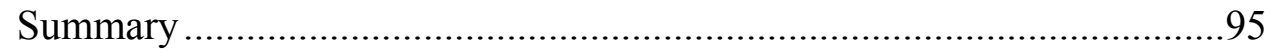

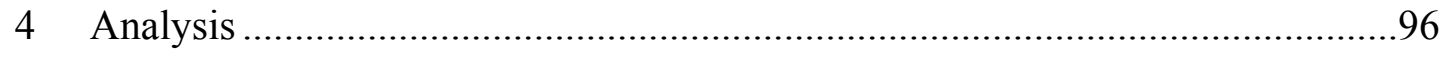

Introduction.................................................................................96

Review of Research Goals/Questions and General Design of the

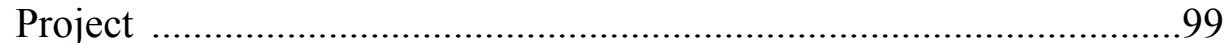

Development and Implementation (Field-Testing) of the PBL Project: Description of the Design Team's Experience Through Step Seven of the R\&D Process ....................................................................101

Chapter Summary and Reflection................................................... 143

5 Conclusions, Speculations, and Recommendations for Leadership .................147

Introduction............................................................................... 147

Overall Conclusions and Assessment of the Experience........................149

Speculations About Future Research, Development, and Use of the

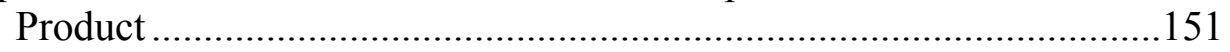

Recommendations for Leadership ...............................................157

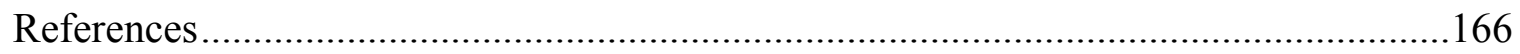

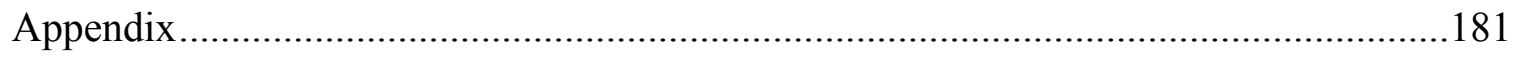




\section{List of Tables}

Table Page

1 The Four Key Questions PLCs Must Ask ....................................................25

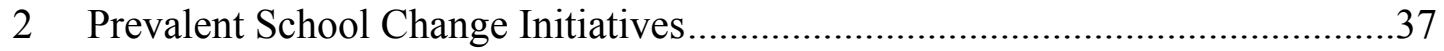

3 The Guiding Principles Behind Prevalent School Change Initiatives..................38

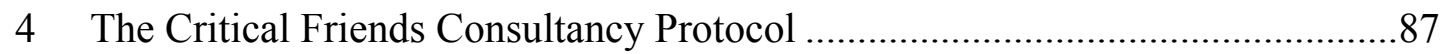

5 Coding and Developing Category Systems ............................................ 90

6 PLC Survey: What is Your Current Reality? ................................................114

7 Evaluation Rubric for Development of the Estacada PLC Handbook ...............120

8 Roadmap for the Estacada PLC Handbook Main Field-Test ...........................123

9 Survey of the Effectiveness of the Estacada PLC Handbook...........................136 


\section{List of Figures}

Figure $\quad$ Page

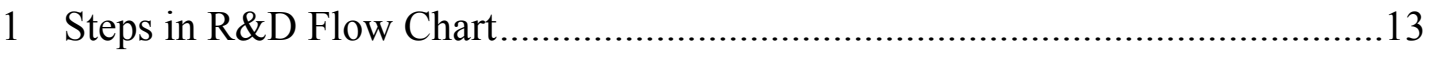

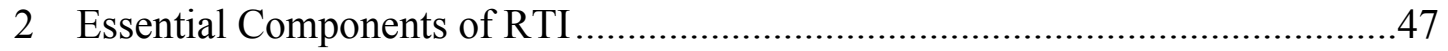

3 PBIS Continuum of School-Wide Support..................................................49

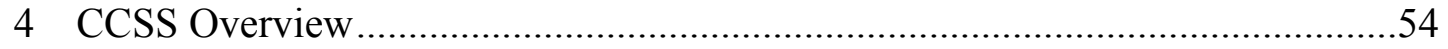

5 Proficiency-Based Learning: Key Questions of the Assessment for Learning

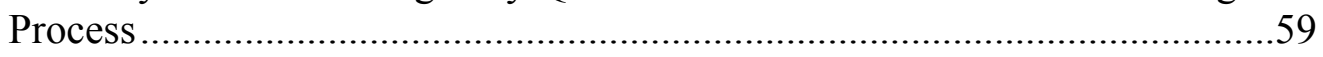

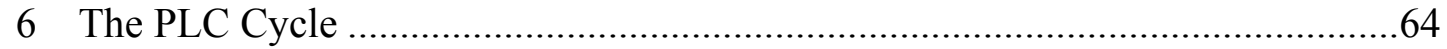

7 The Various Roles of the Principal...............................................................65

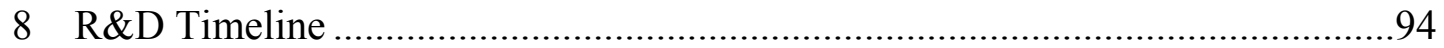




\section{Preface}

As a principal with 9 years of administrative experience, I have always embraced the idea of teachers working together in collaborative teams. I have been a principal at both the elementary and secondary levels, and staff collaboration through the professional learning community (PLC) process has been beneficial at both levels. In the small, rural district where I work, the principals wear several different hats. For example, I am currently an elementary principal and the director of the English Language Learner and migrant programs for the district. In addition, our administrative team has spent much of this school year operating without a superintendent. Because principals in our district have experienced increased workloads, PLC effectiveness has become more crucial. Our former superintendent commented several times about how impressed he was with my ability to guide PLCs. My staff members are known for high levels of PLC collaboration when it comes to identifying essential learning targets, creating and administering common assessments, analyzing student assessment data, and implementing intervention and enrichment opportunities for students. However, while the schools in our district have operated as PLCs for the past 6 years, they have never had a systematic or comprehensive approach to guide the PLC process.

I had a number of concerns as our design team began to formulate its handbook for PLC leadership. For example, how would staff react to us intensifying our tactics to guide the PLC process through the use of a comprehensive handbook? We wanted our staffs to know that we had a great deal of trust in their ability to make decisions that 
benefit students. However, we also wanted to send the message that we would need to systematically outline PLC expectations in order to accomplish all of the work that the state and federal governments were asking of our schools. We had to find ways to empower teachers, yet still establish that there would be distinct boundaries, guidelines, and expectations for PLCs. We had to facilitate a shared vision that demonstrated to them that a more systematic approach to PLC work would ultimately be what is best for all schools. We had to help teachers realize that the refined PLC process would provide opportunities for them to become more efficient and productive in their jobs, and that it would help them complete all of the demanding tasks required of them.

Early release time for PLC work was already in place (students were released 2 hours early every Friday to allow for staff PLC collaboration time). PLC meetings took place once a week. Multiple PLC meetings, involving different groups of people, took place throughout each school simultaneously. Teachers in PLCs worked to address student needs and school change initiatives. We had to make PLC time sacred, untouchable, and focused. Distractions could infringe on PLC time and take away from the intended work. We knew we would need to keep PLC time focused. However, we also understood that, as principals, we would not be able to be in all places at all times to ensure that PLCs were on task. Furthermore, even if we were able to be in all places at all times, the challenge of implementing PLCs would still be daunting. To address this problem, our design team (comprised of the district's principals and one vice principal) created, field-tested, and refined a handbook that guided the work of PLCs in the schools across our district. Throughout our research and development, we had focused 
discussions with each other, conducted structured interviews with teacher leaders, reviewed institutional documentation, and analyzed survey results. The design team was able to establish a handbook for our district-Guiding the Work of Professional Learning Communities: Perspectives for School Leaders. 


\section{CHAPTER 1}

\section{INTRODUCTION}

This study was conducted in the Estacada School District. Estacada is a small, rural town in Oregon, approximately 35 miles east of Portland. Estacada is located in the foothills of the Cascade Mountains and is made up of two elementary schools, a junior high school, a high school, and two charter schools. Estacada provides a small town school environment for 1,783 public school students in its regular district buildings, and approximately 850 students in its charter schools. The district's service area covers a surprisingly large geographic area of more than 750 square miles of rural Oregon countryside in the Clackamas River area, and includes considerable portions of the Mt. Hood National Forest. Fifty-five percent of the district's students are economically disadvantaged and receive free or reduced lunch. In terms of race and ethnicity, the district's students are primarily white, with a growing Hispanic population that currently makes up $8 \%$ of the district.

The Estacada School District laid the preliminary groundwork for Professional Learning Communities (PLCs) in 2004 at its high school, when the principal worked to establish common prep periods for teachers in the same department. The effort to establish common planning and problem-solving time was well received by teachers, but it never fully worked out due to constraints with the master schedule. In 2007, school leaders convinced the school board that uninterrupted collaboration time for PLCs was a necessary adaptation to the district's calendar. As a result, the newly adopted calendar 
established an early release day once per week for students, which allowed teachers to meet as PLCs after the students were dismissed. From 2007-2013, teachers in Estacada collaborated in PLCs, and principals used a similar process to guide PLC efforts. However, it was not until 2013 that the district developed a systematic and comprehensive handbook to guide PLC work in all schools. The PLC handbook helped move collaborative work forward in the small, rural school district and community of Estacada.

The purpose of this study was to create, field-test, refine, and assess the usefulness of the handbook in facilitating the work of PLCs. PLCs are collaborative groups of teachers-usually from the same grade level or subject area-who work interdependently to align curriculum, create and administer common learning assessments, analyze student achievement data, and implement classroom-based interventions and enrichment opportunities for students. PLCs use data to make informed decisions about how to best serve students (DuFour, DuFour, Eaker, \& Many, 2006). In most districts, PLCs are mandated and meet once a week for a minimum of 1 hour. Implemented effectively, PLCs can take schools to new levels of success. However, there are many challenges schools face when trying to implement PLCs. For example, what happens if there is one member of the group who is not engaged in the process? What if the personalities of the people in the group clash? Or what if teacher groupings do not fit together naturally? For example, what if there is only one fifth grade teacher in an elementary building? What if there is only one health teacher in a secondary building? With whom do those teachers work? What about the specialists (PE, music, art, etc.)? 
Into which PLC group do they fit? Moreover, what happens if other outside forces such as planning for Jog-A-Thon, getting ready for Family Fun Night, or setting up for an assembly begin to threaten the PLC time that is deemed sacred and untouchable? Furthermore, what happens if teacher leadership and commitment does not emerge in PLCs? Can PLCs still be effective?

These challenges are common in schools, and they demonstrate how critically important the role of the school principal can be in addressing them. The aim of this study was to develop a handbook that will guide the facilitation and work of PLCs, a particular challenge for school leaders. Typically, there are several PLCs working on different tasks throughout the school. The principal is often not able to attend PLC meetings due to a variety of other responsibilities. School leaders, including principals, need resources and tools to help lead teams when they are struggling with aligning their curriculum, agreeing on common assessments, or just getting along. Furthermore, in most schools, there is basic reform work that is not optional. Mandated or highly recommended initiatives ranging from Differentiated Instruction (DI)/Sheltered Instruction (SI) and Response to Intervention (RTI)/Positive Behavioral Intervention Support (PBIS) to Common Core State Standards (CCSS) and Proficiency-Based Learning are expected and overlay the work of PLCs. This work has to get done, not only to help students succeed, but also to satisfy measures of district and state accountability.

The Estacada PLC handbook provided school leaders with a framework and tools to guide the PLC process. When effective, PLCs lead to improved student achievement and the accomplishment of district and state mandates. The handbook also addressed how 
to maximize time for staff collaboration and set the conditions and parameters for teachers as they look to meet the needs of all students. In addition, the handbook showed how the PLC process can be used to implement prevalent school change initiatives as mentioned above with fidelity. The handbook discussed how to troubleshoot the challenges that take place when people attempt (and perhaps struggle) to work together in collaborative teams. In addition, it noted that a systematic approach needs to be in place when educators collaborate with each other to ensure program consistency for students as they move up through the grade levels (providing both horizontal and vertical articulation among PLCs). Having a systematic approach to PLC work also helps when new staff members come on board, or when staff members move into different teaching positions. Rather than starting with little structure, teachers in new, unfamiliar situations will already have the foundational elements of PLC work explained for them. Furthermore, this handbook helped principals provide review for veteran teachers working in PLCs. Building capacity for teacher leadership broadened the overall leadership base of the school, and helped move PLCs forward in the direction of the shared vision created by the principal and staff.

Again, a major problem and challenge for the PLC process is that school leaders need adequate tools and resources, and the principal is not always available to provide assistance. The problem, however, is much more complicated than this. It is not as though every PLC meeting would be productive even if the principal was readily available. There will inevitably be situations where group dynamics play a role. How can the school principal effectively watch over and guide so many different groups doing multiple and 
different tasks? This problem of not having adequate PLC tools and resources will be addressed in the handbook. Moreover, the handbook will also recommend that the district administrative team works in a district-level PLC of its own to ensure that all of the district's priorities and expectations are clear and aligned.

This study of PLC work differs from that of the DuFour et al. (2006)-the wellrecognized authors of PLC literature-because it specifically targets principals who lead the PLC process, and how they can involve school community stakeholders. Cultivating school community leadership is an important aspect of what principals must do. Because improvement of a school's performance frequently involves doing things differently from how they have been done in the past, teacher leadership also requires an investment in the school improvement process. An important characteristic of a teacher leader is expertise and skill in engaging others in complex work. Teacher leadership also entails an unwavering passion for the core mission of the school and the courage to confront obstacles to achieving that mission (C. Danielson, 2006). Darling-Hammond and McLaughlin (1995) noted that principals helping teachers rethink practice necessitates professional development that involves teachers in the dual capacities of both teaching and learning and creates new visions of what, when, and how teachers should learn. This statement clearly implies that school principals need to use the PLC process to coach their teachers toward this new way of learning and collaboration.

In the workplace, most problems are solved collaboratively by teamwork. We all have different talents or multiple intelligences (Gardner, 1983). We all have strengths and we all have weaknesses. Why not work together to capitalize on the talents of the group 
in an effort to learn from one another and accomplish goals? PLCs tap into this concept, and the results ultimately benefit the students in our classrooms. It is crucial for principals to utilize the talents of their teachers and put them in positions where they can succeed. If teachers are put in positions to succeed, students will benefit because the data generated from PLC work regarding student achievement will be sound and reliable. The principal must find a way to have a direct impact on how, when, and where teachers learn via the PLC process. How are school principals able to manage all of the different (and perhaps clashing) personalities of their staff? Additionally, how can group members' talents be utilized in ways that are positive rather than combative? The handbook will provide tools for principals attempting to guide the PLC process. In the words of DuFour et al. (2006), some of the main proponents of PLCs:

The current emphasis on shared decision-making, dispersed leadership, staff empowerment, collaboration, and collegiality has tended to obscure another harsh reality about substantive change: It demands the sustained attention, energy, and effort of school leaders. The idea of bottom-up reform is great, but it is unrealistic to assume that one day a group of educators gathered in the faculty lounge will suddenly begin to re-examine the basic assumptions, beliefs, and practices that constitute the culture of their shared school. (p. 191)

What DuFour et al. stated has significant implications for the PLC handbook; it demonstrates that the principal is truly the key leader of PLCs. If the principal's leadership is sound, and is able to cultivate teacher leadership, PLCs can be implemented effectively.

PLCs allow for collaboration and teacher leadership in a variety of ways, including: 
- Identifying high priority standards-the most essential learning targets from the CCSS

- Creating and administering common formative learning assessments (different from the Oregon Assessment of Knowledge and Skills (OAKS) and SMARTER Balanced, which are summative state assessments)

- Analyzing the student achievement data from common assessments

- Planning for classroom-based intervention and enrichment opportunities for students using the data from their common assessments

- Peer coaching opportunities, in consultation with the building administrator, that allow teachers to:

o Observe fellow educators teaching and modeling lessons

o Team-teach in certain situations

o Provide feedback to peers about what they noticed during a lesson observation

PLCs usually take the form of weekly meetings among small groups of like teachers. However, they can also resemble peer coaching (Pajak, 2000). This is not a new model, rather an extension of the traditional weekly PLCs meetings through peer coaching. In peer coaching, the members of a PLC will study a particular instructional strategy or model of teaching and discuss it. Then, teachers will observe a demonstration of the new strategy by someone who is expert in its use (either live or on video). Teachers then collaboratively plan mini-lessons and prepare materials to apply the new strategy with other teachers who play the role of students. Finally, teachers introduce the new 
teaching method in their regular classes, with partners and coaching teams (PLCs) providing feedback, assistance, and companionship. Joyce and Showers (1995) have the belief that schools should strive toward a coaching environment in which all teachers view themselves as each other's coaches.

Inspired by the medical-rounds model used by physicians, Elmore, City, Fiarman, and Teitel (2009) have promoted a new form of professional coaching known as instructional rounds networks. Through this process, educators develop a shared practice of observing, discussing, and analyzing learning and teaching. Providing companionship is critical, as teachers embark on the difficult and risky process of learning something new. Coaching allows teachers to reflect on an idea, check perceptions, share successes and frustrations, and solve problems. Coaching also lets teachers know that their feelings and failures are not unique, and that their colleagues may be experiencing similar difficulties. While the well-known aspects of the PLC process such as identifying learning targets, administering common assessments, analyzing assessment data, and planning intervention and enrichment activities are crucial, the peer coaching aspect of PLCs should not be ignored. Traditional PLCs as well as PLCs with the added feature of peer coaching can help principals cultivate teacher leadership, meet student needs, and address school change initiatives.

\section{Statement of the Problem}

There is a great deal of research on PLCs (DuFour, DuFour, Eaker, \& Karhanek, 2004; Marzano, Waters, \& McNulty, 2005; Reeves, 2006; Schmoker, 2006; Senge, 1999; Wenger, 1998), yet there is no clear framework to help principals effectively guide and 
facilitate PLCs within a school (Vescio, Ross, \& Adams, 2006). Many researchers and practitioners agree that PLCs are critical to the overall success of a school, yet the role of the principal is rarely talked about in PLC literature. If the principal is the primary leader of the school, it only makes sense that the principal has an active role in guiding this allimportant process. The literature is silent on this role.

In recent discussions on collaboration in schools, a prominent issue has been whether the principal should be heavily involved with the work of PLCs. On the one hand, some argue that PLCs should be teacher-driven, and that the principal should be detached. From this perspective many agree that teachers are the experts in their respective content areas, and that the principal should not decide or direct content. On the other hand, there are some-including myself-who argue that the principal is the primary instructional leader of the school. Hence, if PLCs are the main vehicle for school reform, it makes no sense for the instructional leader-the principal-to have a detached role. According to this view, at the school level, it is the principal who ultimately ensures the necessary conditions that allow PLCs to flourish.

The issue is whether the principal is willing to stand on the sidelines as an observer of school reform, or whether able to choose to be an active participant and leader in guiding school reform that will serve to benefit students. Even though a school principal cannot be in all places at all times, the principal is still be the most essential leader in the PLC process in the school (DuFour et al., 2006). A principal does this by establishing procedures for how PLCs should operate. A principal can also maintain communication with each PLC by connecting regularly with teacher leaders. If 
facilitation is effective, the principal will be able to harness the strengths of PLCs, which range from collaborating with one another, sharing effective teaching strategies, and analyzing student achievement data, to implementing mandated interventions and providing enrichment activities. In addition, the principal will also be able to help troubleshoot and address challenges such as weak teacher leadership, poor group dynamics, and a lack of focus on critical work before they become overly problematic. The handbook will help principals navigate through these potential problems by providing activities that can be used with staff.

\section{Purpose and Significance of the Study}

The purpose of this study was to create, field-test, refine, and assess a handbook to provide tools to facilitate the leadership of PLCs. Principals used the handbook to promote and lead PLCs. The handbook provided activities, figures, graphic organizers, tables, and charts, which principals used with their staffs to ensure that the PLC process moved forward in a direction that was congruent with the school's mission of increased student learning. The activities were simple and user-friendly so that they did not overwhelm teachers. However, the activities did not oversimplify the process so that the important aspects of the necessary work were ignored. It should be noted that principals using the handbook with their staffs symbolized change. People often struggle with change, but change is necessary when schools strive to improve their results.

Some staff members may resist any type of school reform effort coming from administration. However, this study maintains that a certain degree of top-down leadership is necessary in order for school reform to truly take place. The idea of grade 
level or content area departments getting together to talk about what students should learn is nothing new. These natural configurations of groups have gravitated to each other for several years, and they have been involved in PLC-type work. However, it is unlikely that a school-wide approach could ever be fully realized, implemented, and sustained without effective leadership from the principal. Some might object to the principal insisting that departments collaborate; particularly the inevitable resisters who like to shut their doors and be left alone. However, this study maintains that teachers can no longer work as independent contractors; they must work together. The issue of collaboration through PLCs is relevant because many mandates and measures for accountability must be addressed. Teachers and principals need to rely on each other not only to satisfy the federal government, the state, and the district, but more importantly to do what is best for the students they serve. Facilitating school change requires more than an invitation. The challenging of deep assumptions inherent in changing the culture of a school requires principals to assert their influence; this can occur through the PLC process (Ubben, Hughes, \& Norris, 2004).

\section{Research Methodology}

The research framework for this study was grounded in problem-based learning (PBL; Bridges \& Hallinger, 1995). The PBL design takes the findings generated by the basic and applied research and development (R\&D; Borg \& Gall, 1989) process and uses them to build and refine tested products that are ready for operational use in schools. The Estacada design team collected data while developing, field-testing, and refining the handbook. The design team coded the data, highlighting key patterns and themes 
(Creswell, 2005). They made observations and recommendations for future practice based on the coded data, to determine what elements of the handbook worked well for district principals guiding PLC work in their schools. Engaging in this PBL project allowed the team to partake in critical synthesis, systematic inquiry, and application of domain-relevant knowledge. For working professionals, PBL projects provide productive linkages between research, theory, and practice. In short, the design team believed that school principals would appreciate and benefit from this study's findings and recommendations for leadership of the PLC process.

This study's PBL project was constructed under a model referred to as R\&D. Borg and Gall (1989) described R\&D as a process used to develop and validate educational products. The PLC handbook was developed, field-tested, and refined so that it would be ready for operational use in schools. Research is a systematic inquiry to describe, explain, predict, and control the observed phenomenon, which this study documented in terms of how successfully the product actually guided the PLC process in schools and led to student benefit. One of the main purposes of a PBL product is to test the validity of one's explanations. Gall, Borg, and Gall (1996) submitted that the heart of the research is not on statistics, but in the thinking behind the research. Specifically, what evidence can one provide and support that will persuade people to accept the argument presented. Gall et al. (1996) noted that research can work to provide description, prediction, improvement, and explanation. The researchers need to identify a significant problem. In this case, the problem identified was the fact that PLCs are challenging for schools to implement effectively. 
This study provides an overview of the existing literature to fully understand the problem of PLC leadership. Reviewing the literature has two purposes. First, it builds a knowledge base on the topic under exploration and provides a platform for deeper understanding. Secondly, the study contributes to the knowledge base as well as the practice of PLC leadership. The study focuses on the principal's role in guiding PLCs, and demonstrates how PLCs can serve as a vehicle for other school reform initiatives. Primary data collection methods include the use of a design team guided by a systematic protocol, as well as follow-up interviews, observations, and the review of pertinent documents (Creswell, 2005). The recognition of subjective interpretation and bias of the information-especially since the information comes from the design team's own districtis critical. The handbook was created, field-tested, and refined through the first seven steps that Borg and Gall (1989) identified in their 10 step R\&D process (see Figure 1).

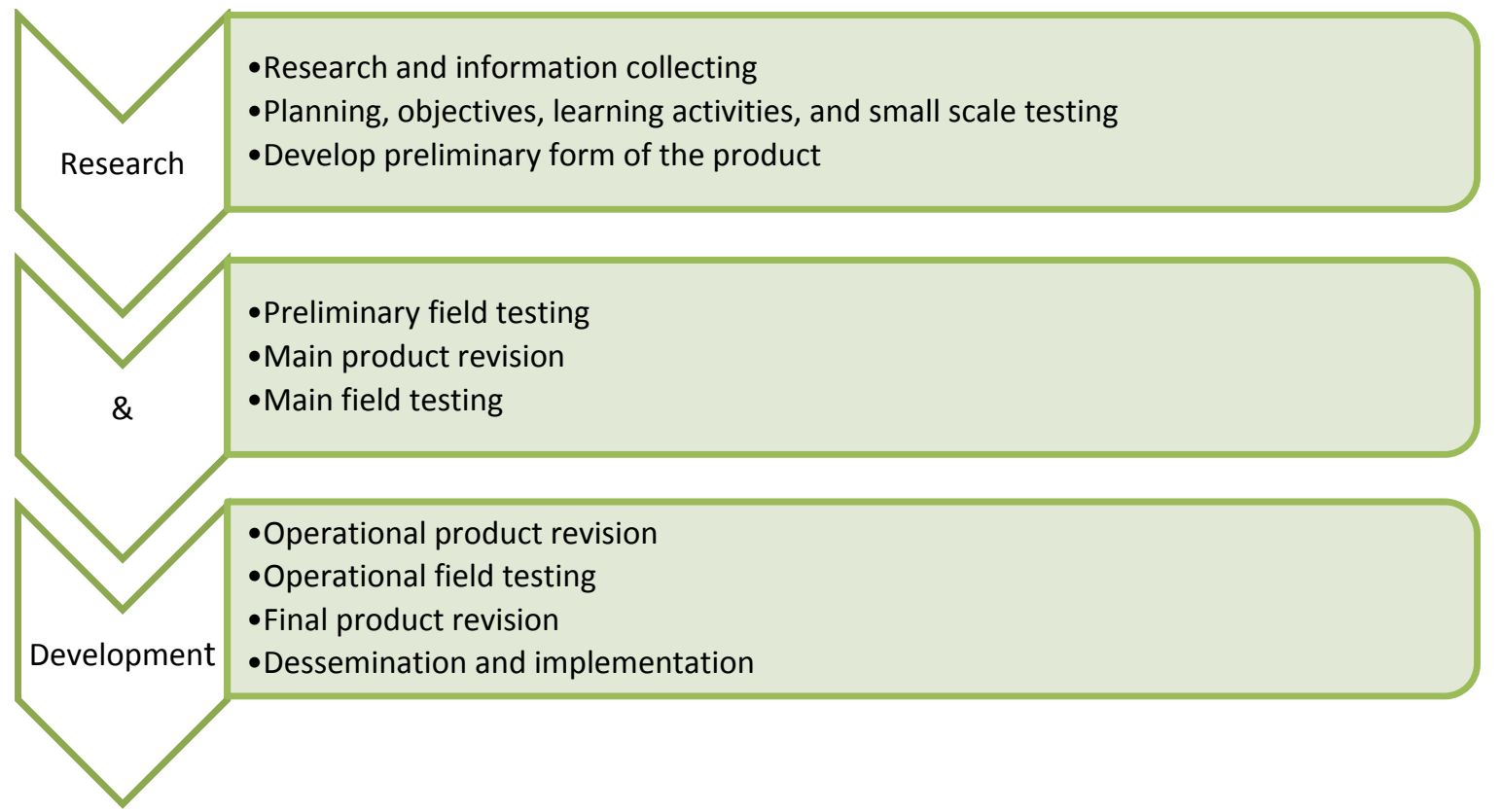

Figure 1. Steps in R\&D flow chart. Source: Borg and Gall (1989, pp. 784-785). 


\section{Summary}

In summary, the PLC process is an important innovation that schools use to inform their decision making and improve student learning. The problem, however, is that implementing PLCs in a school is challenging for a variety of reasons. Teacher leadership is not always strong. Group dynamics often prevent consensus. People do not always stay focused on the intended work. In addition, school principals have large workloads and often serve multiple roles within their district. PLCs are not only desirable, they are necessary in order to do all of the work that schools are required to complete. Because PLC work is so important, the principal should guide the process toward the attainment of its goals. The PLC handbook will help principals maintain contact with their PLCs, and show PLCs how to complete their work. In order to fully understand the problem of principal leadership of PLCs, a review of the literature follows. PLCs, school change initiatives that PLCs can support, and principal leadership will be fully explored.

\section{Definitions of Key Terms}

PLCs (Professional Learning Communities): Team members who work interdependently to achieve common goals linked to the purpose of learning for all (DuFour et al., 2006).

School Change: A process based on various stages, including: pre-initiation and initiation, building commitment, implementation, sustaining change, and evaluation and assessment (Chenoweth \& Everhart, 2002). 
DI (Differentiated Instruction): A framework for addressing learner variance as a critical component of instructional planning (Tomlinson \& McTighe, 2006).

SI (Sheltered Instruction): An approach for teaching content in strategic ways that make the subject matter concepts comprehensible while promoting students' English language development (Echevarria, Vogt, \& Short, 2010).

RI (Response to Intervention): RTI asserts that students who are not responsive to instructional interventions that are effective with most students are in need of timely, frequent, and intensive intervention to accelerate their progress and thereby avoid delays in attaining short-term benchmark and annual, grade level proficiencies (Greenwood, Kratochwill, \& Clements, 2008).

PBIS (Positive Behavioral Intervention Support): The emphasis on school-wide systems of support that include proactive strategies for defining, teaching, and supporting appropriate student behaviors to create a positive school environment (Sailor, Dunlap, Sugai, \& Horner, 2009).

CCSS (Common Core State Standards): A national and common set of expectations across states for what K-12 students are expected to know and be able to do (Adair, 2012).

Priority Standards: Priority standards are needed to simplify the curriculum; to drastically reduce the number of standards to those with the highest priority. A focus on high-priority standards not only optimizes essential learning, it also ensures endurance, leverage, and readiness for the next level (Reeves, 2006; Schmoker, 2011). 
Proficiency-Based Learning: A classroom assessment process that provides a continuous flow of information about student achievement. Proficiency-Based Learning includes identifying achievement targets in advance of teaching (provided in terms students understand) and frequent descriptive feedback provided by the teacher to the student (Stiggins, 2005).

Principal Leadership: The capacity to create and communicate a view of the desired state of affairs that induces commitment among those working in the organization (Ubben et al., 2004). 


\section{CHAPTER 2}

\section{LITERATURE REVIEW}

\section{Introduction}

PLCs are important to a school's success, but implementing PLCs effectively is challenging (DuFour et al., 2006). Principals need accessible and specific resources to help them effectively lead the PLC process in their school. There are numerous problems that need to be navigated while doing this important work. In today's educational climate, many teachers and principals feel overworked (Thompson, Gregg, \& Niska, 2004). Principals, in particular, have much to oversee in terms of managing federal, state, and local mandates and initiatives. Combine increased expectations with a reduction in staff and resources, and principals find themselves in a formidable quandary (Dunklee \& Shoop, 2006). There is so much to do, and nobody can do it alone; this is where PLCs can be utilized. In order to complete the myriad of daunting, required tasks, teamwork is not only desirable, it is essential. There are a number of well-respected researchers (DuFour et al., 2006; Marzano et al., 2005; Reeves, 2006; Schmoker, 2006; Senge, 1999; Wenger, 1998) who feel that in order to complete all of the necessary work, a school must set up a system that facilitates teamwork and shared responsibility through PLCs.

The principal of the school must be the key leader in setting up a system of collaboration guided by a shared vision (Shields, 2003). In addition, the principal will need to be the key mediator and problem-solver as challenges come up throughout the school year-as they inevitably will (Whitaker, 2003). This literature review discusses 
three main areas: (a) PLCs, (b) school change initiatives that PLCs can support, and (c) principal leadership. Ultimately, I synthesize the research on PLCs, school change initiatives, and principal leadership to reveal their implications for the problem the design team is addressing. I discuss gaps in the research literature, as well as the various methodologies employed, and describe what is most significant about the findings for the design team's work.

\section{PLCs}

PLCs are collaborative teams whose members work interdependently to achieve common goals linked to the purpose of learning for all (Eaker \& Gonzalez, 2007). Learning for all is defined as progress and growth for each student, based on the student's individual learning profile (Riehl, 2000). PLCs can take on a variety of forms, but the most recognizable examples include groups such as: single grade teachers (at the elementary level); the math, language arts, science, and social studies departments (at the secondary level)-the resident experts in their respective content areas. It is crucial that school principals tactfully assert their leadership style and priorities into the culture of the school community (Whitaker, 2003). It must be made clear that participating in PLCs is not optional because it is a part of how schools operate (Matthews \& Crow, 2010). It is imperative that PLCs do the important work of identifying essential learning targets, administering common assessments, analyzing student assessment results, and planning for intervention and enrichment opportunities. Studies noted that teachers in PLCs work in collaborative teams to build shared knowledge regarding standards, district curriculum 
guides, trends in student achievement, and expectations of the next course or grade level (Harris \& Jones, 2010; Riehl, 2000; Zepeda, 2004).

PLCs come out of a constructivist framework (Bruner, 1996). Constructivism is based on the belief that learning occurs as learners are actively involved in a process of meaning and knowledge construction as opposed to passively receiving information. Constructivism fosters critical thinking and creates motivated learners. The historical development of PLCs was based on the idea that through dialogue, teachers can form a network of understanding, a community of others with whom they can learn and share through discourse (McLaughlin \& Talbert, 2001). As the idea of teachers working collaboratively in teams has evolved, collaborative focus has shifted from school-wide teams preoccupied with general operations to grade-level and subject-centered teams whose mission is to improve student achievement (Ogawa \& White, 1994).

Van Lare and Brazer (2013) argued that two main weaknesses exist in the literature on PLCs. First, little empirical research is rooted in established learning theory. The absence of a clear, agreed-upon theoretical model used to analyze teacher learning in the PLC setting creates a situation that runs the risk of neglecting the movement's central purpose: teacher learning. Second, PLCs tend to be studied in isolation, with little attention to the context within which they exist. The conceptual framework for this study connects organizational theory and the theories of learning to formulate a hypothesis for how learning could occur within PLCs guided by a comprehensive handbook. Chenoweth and Everhart (2002) found that there is typically a need for smaller group meetings (such as PLCs) and fewer traditional whole group faculty meetings. These small group PLC 
meetings should be focused on an inquiry process (Chenoweth \& Everhart, 2002) or action research process (Schmuck, 2006) as a means or path for working together to critically examine and address a school's major problems or challenge areas. Inquiry and action research appear deceptively simple, but in reality faithfully and thoughtfully following these approaches is one of the most challenging aspects of PLC work and school reform (Chenoweth \& Everhart, 2002).

\section{Strengths of PLCs}

At their core, PLCs are data-informed, standards-driven, and focused on instruction, equity, and results (DuFour et al., 2006). From the numerous studies I read (Cameron, McIver, \& Goddard, 2008; Cochran-Smith \& Lytle, 1999; Garet, Porter, Desimone, Birman, \& Suk Yoon, 2001; Haslam, 1998; Louis, Marks, \& Kruse, 1996; Merriam, 1998; Thompson et al., 2004; Wilson \& Berne 1999), a number of positive results among practitioners engaged in collaborative PLC work have taken place. Some examples of positive results through PLC work included: a demonstrated change in teaching practices, noticeable change in school culture, and evidence of increased student achievement. The studies described internal and external factors that influenced change. The studies also analyzed the selection and implementation of the PLC model. In addition, the studies examined the cycle of transformation that occurred, including interactions between school principals, teacher leaders, and other professional staff as PLCs became institutionalized. Moreover, outcomes that resulted after a period of time of implementation were discussed. 
As a result of this collective inquiry, teachers typically established the priority standards for each unit of instruction and committed to instruct their students in essential learning according to the team's agreed-upon pacing guide (Marzano et al., 2005). Priority standards are defined as the most critical standards within the vast list of CCSS. CCSS are a national and common set of expectations across states for what K-12 students are expected to know and be able to do (Council of Chief School Officers and National Governors Association, 2010). While the CCSS are more refined and focused than previous state standards, these updated standards still require teachers to engage in a process of prioritization to address the most critical content (Adair, 2012). The pacing guide is defined as the scope and sequence of lessons that teachers-PLC members-adhere to so that they are able to guarantee a viable curriculum, analyze student results, and plan for interventions and enrichment in a timely and efficient manner.

Another noted strength of PLCs found in the research was that they assess whether students have learned the essential curriculum. The essential curriculum is defined as the priority standard-the most critical of the CCSS. In the studies reviewed, teachers worked with colleagues to develop a series of common formative assessments that were aligned with priority standards and district curriculum guides. PLCs established the specific proficiency levels that each student was to meet. Teams administered their common formative assessments multiple times throughout the school year and analyzed the results together. Teachers used the results to inform and improve their individual and collective practice, to identify students who needed additional time and support for learning, and to help students monitor their own progress toward agreed-upon priority 
standards (DuFour et al., 2006; Schmoker, 2011). From their research on PLCs, Fulton, Yoon, and Lee (2005) highlighted the following strengths:

- Building and deepening teacher knowledge

- Integrating new practitioners into a teaching community and school culture, which supported the continuous professional growth of all teachers

- Supporting the constant development of the teaching community in the school

- Encouraging a professional dialogue that articulates the goals, values, and best practices of a community

- Exhibiting promising organizational qualities

\section{Challenges of PLCs}

While many positive results were realized through PLCs, several trends noticed across sites were also detrimental to a school's core mission. For example, a lack of assumed teacher leadership was damaging to the PLC process (Cameron et al., 2008; C. Danielson, 2006). Teachers in PLCs must be expected to exert influence beyond their classrooms and play important roles in the larger arena of the school, the district, and the community. As teachers' expertise was recognized, their roles were expanded, and their responsibilities increased. These teachers became more powerful leaders and modelers of learning. Unlike bureaucratic forms of teacher leadership that simply create slots in an already isolating and compartmentalized structure, PLCs offer organic forms of professional leadership that develop intrinsically in connection with systemic organizational change within a school (Darling-Hammond, Bullmaster, \& Cobb, 1995). 
Principals need teachers to assume leadership in the PLC process, and they often will, because PLCs provide a low-cost, sustainable, satisfying, and potentially transformative form of teacher professional development (Snow-Gerono, 2004). However, if the principal is unable to cultivate teacher leadership, the PLC process in the school will suffer. Undoubtedly, it is difficult for the principal to keep asking teachers to assume more responsibility while their class sizes increase and resources dwindle due to state and district economic realities.

Another challenge for implementing PLCs can be an unclear message from the principal. If the principal of the building is not a champion of PLCs (or does not have the knowledge and expertise), and does not guide the work, the negative resisters will ultimately destroy the positive potential of PLCs (Cochran-Smith \& Lytle, 1999; Garet et al., 2001; Wenger, 1998). Principals need to embed the learning mission into the day-today operations of the school (Eaker \& Gonzalez, 2007). Lezotte (1997) identified creating a community with shared values as the fundamental leadership function of the principal in contemporary schools. Instructional leaders must be attentive to that function, and constantly promote, protect, and defend the mission of learning through the collaborative work of PLCs. It will be a challenge for PLCs to succeed if they do not receive sound leadership and a clear message from the principal.

Group dynamics can also be a challenge in PLC work (Haslam, 1998; Louis et al., 1996; Shields, 2003). Combative or toxic relationships within the group can destroy PLC endeavors. Interpersonal tension often makes people uncomfortable and teachers are no exception (Goulet, Krentz, \& Christiansen, 2003). Teachers need to be trained by the 
principal to work collaboratively in PLCs. Hargreaves (2000) explained that too often, conflict in schools is seen as a problem, not an opportunity, where purposes are threatened, competence is questioned and the undertones of status and power strain the fragile bonds that hold teachers together. When professional criticisms are interpreted as personal attacks, feelings of frustration often surface. Distrust poisons the collaborative process, leaving members unwilling to tolerate differences, and unable to trust each other's motives. In fact, teachers often describe themselves as feeling devastated and angry during group work (Hargreaves, 2001). By instituting PLCs, the principal is increasing the frequency and intensity of teachers' interactions, which increases the potential for conflict because as members work more closely with each other to develop their shared practice, fewer assumptions are left unchallenged (Wenger, 1998).

An example of a study that explored the high-frequency interactions of teachers was conducted by Dooner, Mandzuk, and Clifton (2007), who used Weick’s (1979) model of means convergence to analyze the social dynamics of a group of seven middle school teachers from one suburban middle school over a two-year period as they attempted to implement Egan's (1997) theory of Imagination and Learning in their PLCs and teaching practice. All teachers in the study believed that group activities required trust. Otherwise, they felt that the potential conflicts arising from the members' different work ethics, personal abilities, and quality of work would stifle the group's collaborative efforts. The results of the two-year study indicated that the participating teachers found the open and forthright nature of their PLC discussions essential in realigning individual behavior to the group's goals. The members indicated that while there was tension, the 
group ultimately remained committed to "getting the job done" (Dooner et al., 2007). The handbook will engage PLCs in activities that help them build consensus, make group decisions that will lead to student benefit, and "get the job done."

Another pitfall that PLCs encounter is a lack of focus on instructional content and student learning. A principal must set the conditions for PLCs so that they do not get off task. The principal's role in PLCs goes beyond simply assigning individuals to teams. Principals need to create processes to ensure that teams focus on the critical questions associated with student learning (see Table 1).

Table 1

The Four Key Questions PLCs Must Ask

\begin{tabular}{|l|}
\hline Questions \\
\hline 1 - What do we want our students to learn? \\
\hline 2 - How will we know they have learned it \\
\hline 3 - What will we do for students who have not yet demonstrated proficiency on standards? \\
\hline 4 - What will we do for students who have already demonstrated proficiency and are ready to move on \\
\hline
\end{tabular}

Source: adapted from DuFour et al. (2006, p. 21).

The Dooner et al. (2007) study referenced above found that some teachers reported group members would wander in and out of their discussions, taking valuable time away from their PLC work. The principal must leave no doubt as to what the basic, nonnegotiable, structural components of PLCs are (DuFour et al., 2004; Thompson et al., 2004; Wilson \& Berne, 1999). These components should be in place from the very beginning of the school year so that precious time is not wasted. Hence, PLCs should not 
waste time on process-oriented issues, such as wondering what it is they are supposed to be doing next. Yet, when asking people to collaborate with limited time and reduced resources, issues like group process and management are critically important, which is another reason clear leadership from the principal is necessary. If optimal conditions are established by the principal, PLCs should be on task and focused on student learning, determining which students need intervention and which students need enrichment. Ideally, the PLC process provides time for teachers to complete all of what is asked of them, and it also provides a way for them to lean on one another and learn from each other. In order to avoid the challenge of off-task behavior, principals can keep PLCs on task and focused on student learning by emphasizing the four key questions referenced above.

\section{CollaborationThrough PLCs}

When it comes to the topic of collaboration, most studies readily agree that working in a team is beneficial (Hughes \& Kritsonis, 2006; Vescio, Ross, \& Adams, 2008). Where this agreement becomes a challenge is around the issue of autonomy. Whereas some teachers are convinced that collaboration benefits the school as a whole, others maintain that collaboration stifles their individual creativity and personal freedom to teach their students the way they see fit. Although most of them do not say so directly, some teachers give the impression that they believe they are independent contractors. They do not want to make their work public. They want to be left alone. While the majority of staff members do not feel this way, it has been proven to be the case for the minority (Cameron et al., 2008; Cochran-Smith \& Lytle, 1999; Haslam, 1998). 
Another challenge is that working in isolation limits rigorous and authentic feedback (Schmoker, 2011). Accordingly, PLC members are expected to share student and teacher work in order to give and receive feedback. PLC members then offer suggestions on how to improve practice. Authentic feedback cannot take place if teachers are unwilling to fully commit to the PLC process. Some teachers fear that they will look inferior when compared to other educators. There can also be an underlying competitiveness among PLC members (DuFour et al., 2004). Teachers may not want to share their successful strategies with other teachers for a variety of reasons. In his empirical study of PLCs in secondary schools, Bezzina (2006) noted that PLC meetings revealed interpersonal issues and differences, as well as decision-making issues. The difficulty of getting people to learn how to accept different opinions and to view reality in different ways was highlighted by a comment made by one of the school's principals:

Teachers and management need time to accept positive criticism, to learn that other people may hold different opinions and that we need to start opening up. Democratizing the decision-making process is fraught with difficulties. (p. 163)

Furthermore, it may sometimes simply be a case of one teacher not liking another and not wanting to share for that reason alone (Cook \& Yanow, 1996; Louis et al., 1906; Owens, 1998). The school principal must step in to resolve these situations and find ways to alleviate fears of inferiority and competitive tension. The principal should promote PLCs as a means to help teachers improve their practice and raise student achievement (Matthews \& Crow, 2010). As the leader of the school, the principal may need to address PLC groups as a whole, or may need to meet with certain individuals who are 
contributing to a toxic working environment among the PLC to get the group back on the right track.

As a way to promote positive relationships in PLCs, principals can and should encourage peer coaching; this method is often underdeveloped in schools (Joyce \& Showers, 1995). It is one thing to require teachers to work together in a PLC during a meeting, but it is another to have a teacher open themselves up to criticism by having another teacher observe how that teacher interacts and facilitates learning when students are present. Joyce and Showers (1995) claimed that schools should strive toward a coaching (or co-teaching) environment in which all teachers view themselves as each other's coaches. Most teachers would say they are comfortable and willing to have anyone observe them at any time. Yet, do they really mean it?

When classroom teachers are observed by someone, it can make the observed teacher feel quite vulnerable. These fears are arguably the most significant obstacle in terms of increasing collaboration through peer coaching in a school (Bezzina, 2010). For example, Egodawatte, McDougall, and Stoilescu (2011) conducted a Collaborative Teacher Inquiry project, which was aimed at increasing the quality of learning of Grade 9 applied mathematics, and improving professional development opportunities for teachers. A total of 11 schools participated in the project, which spanned over three semesters. Many teachers involved in the project did not have prior experience of co-teaching or coplanning, nor did they have much experience collaborating with their colleagues. The collaboration involved team planning of a lesson, and then one teacher delivered the lesson with others observing. Each co-teaching opportunity was followed by an 
opportunity to debrief and address issues found in the delivery of the lesson. While many teachers were hesitant at first in team reflection, teachers indicated that they valued their new co-teaching experience as part of their professional development.

When a principal formally observes a teacher, it is evaluative. When a teacher observes another teacher, it is by definition non-evaluative peer coaching. In theory, both approaches are beneficial. In teacher observations, however, the observing teacher will gain ideas and techniques from the teacher being watched. In turn, the teacher being observed will benefit from the feedback received. Providing non-evaluative support for teachers through peer coaching is critical, as teachers embark on the difficult and risky process of learning something new (Pajak, 2000; Stiggins, 2005). Coaching allows teachers to reflect on an idea, check perceptions, share successes and frustrations, and solve problems. Peer observation and coaching is a valuable piece to the PLC process that many schools overlook. If principals value this type of collaboration, they will need to make instructional risk-taking safe. Principals will also need to create conditions to make the PLC peer coaching process work by providing release time, arranging for substitutes, and covering classes (Scribner, Cockrell, Cockrell, \& Valentine, 1999). Defenders of teachers working in isolation assert that working collaboratively in the PLC process stifles their individual autonomy and creativity. This assertion is contradicted by their claim that there is too much work and not enough time or resources to do it, because working together in a PLC helps teachers become more efficient and productive (Little, Gearhart, Curry, \& Kafka, 2003). The PLC process is about streamlining the work that needs to be done by sharing the workload among PLC 
members. Completing all of the work required for various initiatives and mandates will be made easier by leaning on one another in PLCs. The benefits of teamwork will always prevail over the isolated, disconnected efforts of individuals (Matthews \& Crow, 2010;

Ubben et al., 2004). School principals cannot leave success-closing the achievement gap, meeting educational growth targets, making adequate yearly progress-to chance. Principals must make it clear for staff that collaborating is not optional-in fact, it is the only chance we have.

\section{The Effectiveness of PLCs}

Research indicates that there will be an increase in the sharing of ideas among staff, as well as a tighter alignment of the curriculum, if PLCs are implemented effectively (DuFour et al., 2004; Marzano et al., 2005; Reeves, 2006; Schmoker, 2006; Senge, 1999; Wenger, 1998). DuFour et al. (2004) have conducted research on PLCs using case studies, both normative and anecdotal. Their research has focused on the organizational elements necessary to make PLCs possible and effective for teachers. Marzano et al. (2005) have discussed PLCs using meta-analyses as the basis for their writings, and have focused on the aspects of establishing curriculum through PLCs, as well as focusing on the assessment data that is analyzed by PLCs. Reeves (2006), Schmoker (2006), Senge (1999), and Wegner (1998) have also studied PLCs extensively; their main finding is that PLCs can be effective in strengthening school culture and staff morale. Historically, teaching has been a solitary, and sometimes lonely, career choice. Past norms have left individual teachers to determine what is best for the students with whom they work. 
Students learn at different rates, and depending on which school a student attends and the assigned teacher(s), their experiences will differ greatly. While we would like to think that all teachers are equally capable and talented, we know that is not true. It is unfortunate that "chance" has been a key ingredient to certain students' success (Marzano et al., 2005). A structured, systematic approach that supports PLCs must prevail-to take "chance" out of the equation as much as possible. Does this mean that we want teachers teaching the same problem, on the same page of the textbook, on the exact same date, at the exact same time as their counterparts? Certainly not, but some significant congruence in the general pacing guide should exist. The overall scope and sequence should be similar, and collaboration among colleagues has to be an essential part of PLC work (Schmoker, 2006).

In terms of effectiveness, PLCs have been proven to lead to higher levels of student achievement. Mokhtari, Thoma, and Edwards' (2009) case study of Westwood Elementary School (Ankeny, Iowa) reviewed data 2 years following the establishment of PLCs. The school showed significant improvement in student reading performance across all grades. A sampling of the data describing improvements included:

- Ninety-six percent of kindergarteners ended the 2008-2009 school year able to read at or above grade level, with only marginal differences between races and income groups.

- The percentage of first-grade students who achieved a proficient score on reading comprehension rose to $94 \%$ in the spring of 2009 from $87 \%$ in the fall semester of 2008 . 
- The percentage of second-grade students who achieved a proficient reading comprehension score rose to $88 \%$ in the spring from $61 \%$ in the fall semester.

- In third grade, $92 \%$ of the students tested proficient or advanced in reading on state tests compared to only $85 \%$ in the previous year.

- In fourth grade, $95 \%$ of the students tested proficient or advanced in reading on state tests compared to only $90 \%$ in the previous year.

- In fifth grade, $95 \%$ of the students tested proficient or advanced in reading on state tests compared to only $80 \%$ in the previous year.

While these results are positive, data from one school is not compelling evidence that PLCs raise student achievement. However, with respect to the use of data for instructional decision making, research tells us that teacher engagement in ongoing collaborative data review and reflection leads to substantive changes in instruction, which can, in turn, result in significant improvements in student achievement (Reutzel, Cooter, \& Blake, 2008).

As further evidence, a case study by Phillips (2003) documenting the efforts of a middle school engaged in PLCs reported that achievement scores increased dramatically over a 3-year period. More specifically, in this middle school, ratings on a statewide standardized test went from "acceptable" in 1999-2000 with 50\% of the students passing subject area tests in reading, writing, math, science, and social studies, to "exemplary" in 2001-2002 with more than $90 \%$ of the students passing each subject area test. In addition, Vescio et al. (2008) submitted that their collective results offer an unequivocal answer to the question about whether the literature supports the assumption that student learning 
increases when teachers participate in PLCs. The answer, according to Vescio et al., is a resounding and encouraging yes. The evidence on PLCs as they relate to student achievement is certainly promising and emerging, but still is not conclusive. However, the organizational benefits of PLCs are widely accepted and rarely questioned.

The research overwhelmingly indicates that components of the PLC process are essential to facilitate effective collaboration in schools (DuFour et al., 2006). This model is a continuous improvement cycle that PLC team members go through as they move from one unit of instruction to another throughout the school year. While this continuous cycle is widely accepted, there are certainly areas in the research that need more exploration. It is difficult to pinpoint PLCs as the main reason a school succeeds or fails because of the myriad of variables that contribute to a school's overall achievement. According to Vescio et al. (2006), the following kinds of studies are still needed regarding PLCs:

- Studies that document changes in teachers' perceptions of the professional culture of the school

- Longitudinal observational studies that document changes in teaching practice as teachers work in PLCs

- In-depth case studies of changes in teaching practice and student achievement for sample teachers working in PLCs

- Qualitative documentation of the nature of the work teachers do as they analyze student work and how this changes over time 
- In-depth case studies of changes in student learning for sample students in classrooms of teachers working in PLCs

- Documentation of changes in student achievement over time (as teachers participate in PLC work)

Based on what I have read, I agree that these areas in the research need more exploration. Fortunately, these areas of study can be addressed through the action research of a principal working in the field through a PBL project. My contributions to the greater PLC research will touch on many of the gaps listed above. However, the primary focus of my study is to highlight the administrative leadership necessary to ensure success for PLCs. The principal needs to develop the skill of outlining the expectations of PLCs. The principal should entrust PLCs to concentrate on areas where change is most possible and where change will make the most difference for school improvement. The principal's responsibility is to communicate and develop understanding with staff so that PLCs can complete their work and achieve desirable results (Matthew \& Crow, 2010; Ubben et al., 2004).

\section{School Change Initiatives}

There are four initiatives prevalent in schools and districts across Oregoninitiatives that principals cannot ignore. The review of literature discussing current school change initiatives was significant because with dwindling resources and a premium on time, teachers may struggle to ensure that these initiatives are being adequately addressed in their schools. PLC work time (when students are not present) can help teachers address school change initiatives. PLC time can help address the challenge of limited time by 
providing built-in teacher work time on a consistent basis throughout the school year. PLC work can also help teachers address the challenge of dwindling resources because teachers can come together and share the resources that they have acquired individually. PLCs can be the vehicle for addressing school change initiatives, all of which are intended to benefit students. However, it will take astute leadership by the principal to address these initiatives through the PLC process and honor the additional responsibility felt by teachers. It is expected that schools implement the following programs and initiatives:

- DI (Tomlinson \& McTighe, 2006); SI (Echevarria et al., 2010)

- RTI (Ogonsky, 2008); PBIS (Sailor et al., 2009)

- $\operatorname{CCSS}($ Gewertz, 2013)

- Proficiency-Based Learning (Bakula, 2010; Stiggins, 2005; Wormelli, 2006) Comprehensive school change takes time. Principals should expect to see evidence of comprehensive school change by the third year of a sustained effort. Chenoweth and Everhart (2002) highlight five major assumptions, which comprise the backdrop for the stages of their comprehensive school change process. The five major assumptions include:

- Change must focus on improved student learning

- School change must be comprehensive, not piecemeal

- Effective school change demands shared leadership

- All relevant stakeholders must be involved in the change process

- Effective change means changing school cultures 
In order to establish PLCs as the vehicle for comprehensive school change, assumptions of school change must be evaluated. In addition, stages of comprehensive school change (such as pre-initiation and initiation, building commitment, implementation, sustaining change, and evaluation and assessment) must be adhered to and guide the work of PLCs. Principals assure that PLCs are part of the standard operating procedures of the school as a whole. While all of the assumptions mentioned above are relevant to PLCs, the one that stands out in particular is that of shared leadership. Principals are the primary leaders of a school. However, teacher leadership is also necessary for PLC success, and is a prerequisite for long-lasting school change and improvement (Sergiovanni, 1995).

Collaborative work groups in which leadership is shared have the best chance of being successful through the change process. Principals need to balance a "loose/tight" leadership philosophy regarding PLCs. "Loose/tight" leadership refers to the fact that principals may defer to teachers in numerous situations (loose), but the principal will remain the instructional leader of the school and will have ultimate veto power (tight) (DuFour et al., 2006). If a comprehensive PLC framework is understood and followed, it is reasonable to expect that school change initiatives can be addressed. Table 2 outlines the founders and key goals of the current, widespread school change initiatives that can be addressed through PLC work. 
Table 2

Prevalent School Change Initiatives

\begin{tabular}{|c|c|c|c|c|c|c|}
\hline $\begin{array}{l}\text { School } \\
\text { Change } \\
\text { Initiatives }\end{array}$ & $\begin{array}{l}\text { Differentiated } \\
\text { Instruction } \\
\text { (DI) }\end{array}$ & $\begin{array}{l}\text { Sheltered } \\
\text { Instruction } \\
\text { (SI) }\end{array}$ & $\begin{array}{l}\text { Response to } \\
\text { Intervention } \\
\quad(\mathrm{RTI})\end{array}$ & $\begin{array}{c}\text { Positive } \\
\text { Behavioral } \\
\text { Intervention } \\
\text { Support (PBIS) }\end{array}$ & $\begin{array}{l}\text { Common } \\
\text { Core State } \\
\text { Standards } \\
(\mathrm{CCSS})\end{array}$ & $\begin{array}{l}\text { Proficiency- } \\
\text { Based } \\
\text { Learning }\end{array}$ \\
\hline Founders & $\begin{array}{l}\text { Tomlinson \& } \\
\text { McTighe } \\
(2006)\end{array}$ & $\begin{array}{l}\text { Echevarria, } \\
\text { Vogt, \& } \\
\text { Short (2010) }\end{array}$ & $\begin{array}{l}\text { Buffum, } \\
\text { Mattos, \& } \\
\text { Weber } \\
(2009), \\
\text { Organsky } \\
(2008)\end{array}$ & $\begin{array}{l}\text { Sailor, Dunlap, } \\
\text { Sugai, \& } \\
\text { Horner (2009) }\end{array}$ & $\begin{array}{l}\text { Council of } \\
\text { Chief State } \\
\text { School } \\
\text { Officers and } \\
\text { National } \\
\text { Governors } \\
\text { Association } \\
(2010)\end{array}$ & $\begin{array}{l}\text { Stiggins } \\
(2005), \\
\text { Wormelli } \\
(2006), \& \\
\text { Bakula (2010) }\end{array}$ \\
\hline Key Goal & $\begin{array}{l}\text { To enable } \\
\text { learners at } \\
\text { multiple } \\
\text { levels and } \\
\text { abilities to } \\
\text { benefit from } \\
\text { instruction }\end{array}$ & $\begin{array}{l}\text { To explicitly } \\
\text { teach } \\
\text { functional } \\
\text { language } \\
\text { skills such } \\
\text { as how to } \\
\text { negotiate } \\
\text { meaning, } \\
\text { confirm } \\
\text { information, } \\
\text { argue, } \\
\text { persuade, } \\
\text { and disagree }\end{array}$ & $\begin{array}{l}\text { To introduce } \\
\text { a problem- } \\
\text { solving } \\
\text { process, } \\
\text { whose } \\
\text { foundation is } \\
\text { the provision } \\
\text { of systematic, } \\
\text { research- } \\
\text { based } \\
\text { instruction } \\
\text { and } \\
\text { interventions } \\
\text { for struggling } \\
\text { learners }\end{array}$ & $\begin{array}{l}\text { To provide a } \\
\text { framework for } \\
\text { assisting school } \\
\text { personnel in } \\
\text { adopting and } \\
\text { organizing } \\
\text { evidence-based } \\
\text { behavioral } \\
\text { interventions } \\
\text { into an } \\
\text { integrated } \\
\text { continuum that } \\
\text { enhances } \\
\text { behavioral } \\
\text { outcomes for all } \\
\text { students }\end{array}$ & $\begin{array}{l}\text { To establish } \\
\text { a common } \\
\text { set of } \\
\text { expectations } \\
\text { across states } \\
\text { for what K- } \\
12 \text { students } \\
\text { are expected } \\
\text { to know and } \\
\text { be able to do }\end{array}$ & $\begin{array}{l}\text { To } \\
\text { communicate } \\
\text { learning } \\
\text { targets up } \\
\text { front to } \\
\text { students, } \\
\text { ensure that } \\
\text { students } \\
\text { receive } \\
\text { continuous } \\
\text { feedback from } \\
\text { their teachers, } \\
\text { and have } \\
\text { multiple } \\
\text { opportunities } \\
\text { to refine } \\
\text { student } \\
\text { learning }\end{array}$ \\
\hline
\end{tabular}

While there are often areas that overlap within these school change initiatives, the salient features are outlined in Table 3 and are expanded upon in the section that follows. 
Table 3

The Guiding Principles Behind Prevalent School Change Initiatives

\begin{tabular}{|c|c|}
\hline Differentiated Instruction (DI) & $\begin{array}{l}\text { - Learning opportunities allow for student choice } \\
\text { - Learning opportunities are matched with students, } \\
\text { individual learning profiles } \\
\text { - Cooperative learning is encouraged }\end{array}$ \\
\hline Sheltered Instruction (SI) & $\begin{array}{l}\text { - Learning opportunities involve the use of visuals and } \\
\text { graphic organizers } \\
\text { - Learning opportunities allow for student-to-student } \\
\text { interaction } \\
\text { - Important vocabulary is front loaded and students } \\
\text { interact in a print-rich learning environment }\end{array}$ \\
\hline Response to Intervention (RTI) & $\begin{array}{l}\text { - Universal screening assessments are used with students } \\
\text { school-wide } \\
\text { - Teachers analyze student assessment data in order to } \\
\text { plan for future intervention and enrichment activities } \\
\text { - Teachers monitor student progress on a regular and } \\
\text { consistent basis }\end{array}$ \\
\hline $\begin{array}{l}\text { Positive Behavioral Intervention } \\
\text { Support (PBIS) }\end{array}$ & $\begin{array}{l}\text { - Learning activities are designed to be preventative, not } \\
\text { reactive } \\
\text { - School-wide learning activities promote desired } \\
\text { outcomes for all } \\
\text { - An emphasis on celebrating positive results achieved by } \\
\text { students, staff, and the school as a whole }\end{array}$ \\
\hline Common Core State Standards (CCSS) & $\begin{array}{l}\text { - Schools place an emphasis on college and career } \\
\text { readiness } \\
\text { - Teachers emphasize reading, writing, speaking, and } \\
\text { listening activities with students } \\
\text { - Literacy and language activities are promoted across the } \\
\text { curriculum } \\
\text { - Teachers focus on priority standards that are weighted } \\
\text { more heavily on state assessments }\end{array}$ \\
\hline Proficiency-Based Learning & $\begin{array}{l}\text { - Learning targets and expectations are communicated to } \\
\text { students at the beginning of units and lessons } \\
\text { - Multiple opportunities exist for students to demonstrate } \\
\text { improvement and proficiency } \\
\text { - Teachers provide relevant and ongoing feedback }\end{array}$ \\
\hline
\end{tabular}

\section{The DI Initiative}

DI refers to the concept of meeting the individual needs of each learner, by customizing instruction. Tomlinson and McTighe (2006) explained that DI is necessary because learners differ in readiness, interest, and learning profile. Vygotsky's (1978) 
zone of proximal development explains that readiness to learn has to do with a learner's proximity to, or proficiency with, particular knowledge, understanding, and skill. Learners must work at an appropriate degree of challenge. When tasks are too difficult for learners, they become frustrated and shut down. When tasks are too easy, they become bored and do not learn, in spite of the fact that they might earn high grades. DI can be addressed through PLCs, and will be highlighted in the handbook. A current method used to differentiate instruction is "I do it, We do it, Ya'll do it, You do it." This method was first known as "I do it, We do it, You do it" (Eisenhart, 2007). The instructional method has been expanded to emphasize the sequential options available for teachers. The instructional method paints a picture of what should be happening in the classroom for teachers attempting to differentiate their instruction.

The "I do it, We do it, Ya'll do it, You do it" DI method includes:

- Teacher-led activities, which include showing examples (I do it)

- Whole class activities (We do it)

- Students working in small groups while the teacher circulates and checks for understanding (Ya'll do it)

- Individual activities followed by teacher feedback (You do it)

Learning happens when a task is a little too difficult for a learner and scaffolding is provided to help the learner span the difficulty. Scaffolding is the support given during the learning process, which is tailored to the needs of the student with the intention of helping individual students achieve their learning goals (Sawyer, 2006). This statement applies to both ends of the spectrum (e.g., the talented and gifted students, the learning 
disabled students, and everyone in between). Instructional scaffolding is a learning process designed to promote deeper learning. It is important for teachers to recognize the kind of scaffolding needed for individual students, and plan for it in their PLCs. Bloom's Taxonomy (Bloom, Englehart, Furst, Hill, \& Krathwohl, 1956) includes six levels. From the lowest developmental level to the highest, the taxonomy includes: remembering, understanding, applying, analyzing, evaluating, and creating. It is important to note that having students at the top of the spectrum use higher levels (applying, analyzing, evaluating, and creating) and students at the bottom of the spectrum remain at the lower levels (remembering and understanding) is not what DI calls for. All students can, and should, think at higher levels. Motivation to learn is decreased when tasks are consistently too difficult or too easy. Hence, it is important for teachers to work together in PLCs to diagnose their students' skill levels and prescribe appropriate tasks (Tomlinson, 2001). Moreover, teachers in PLCs can share their successes and failures around differentiation. By doing so, teachers will gain valuable insights that they would not otherwise have-if not engaged in the PLC process.

Recent studies on teacher effectiveness substantiate the critical role of sound teaching practices, especially differentiation and higher order thinking skills (Wenglingsky, 2000). VanTassel-Baska et al. (2008) conducted a 3-year study, employing an experimental design that compared experimental and comparison teachers' behavioral changes as measured by an observation scale of differentiated teaching strategies. The participants in this study were teachers across six school districts, and they were randomly assigned to an experimental or a comparison group. The major instrument 
used for this study was the Classroom Observation Scale-Revised, which is a scale developed for assessing teachers' instructional practice against expectations derived from best practices in mainstream and gifted classrooms (VanTassel-Baska et al., 2003). The experimental group received ongoing professional development in DI. In year three, the study revealed that the experimental teachers not only surpassed comparison teachers in every behavior cluster at each observation data point, the improving pattern of their instructional practices increased at such a large magnitude, an important instructional competence gap was noticed between the two groups of teachers.

An emerging research base supports DI as a strategy that enables learners at multiple levels of ability to benefit from instruction (Subban, 2006). McAdamis (2001) reported that DI resulted in significant improvement in test scores for low-achieving students across grade levels. In addition to improvements in test scores, another result of DI, according to the studies, is increased student engagement. Johnsen (2003) found that differentiated techniques were engaging and stimulated student interest. Moreover, the advantages of DI based on the learning-style preferences of students of all ages have been documented extensively by more than 40 years of research on the Dunn and Dunn Learning-Style Model (Dunn, 2009), which focused on supporting at-risk students with DI. Multiple experimental research studies conducted with middle level students also reported significantly higher scores on achievement tests when participants experienced instruction congruent with their learning-style strengths (Farkas, 2003). Furthermore, several American schools have reversed poor academic achievement by using DI and 
providing failing students with instructional approaches responsive to their learning-style preferences (Dunn \& DeBello, 1999).

Additional contemporary theories that support DI include brain-based learning and multiple intelligences (Gardner, 1999). Brain-based instruction suggests that students are better prepared neurologically for learning when they are in a comfortable learning environment, appropriately challenged, and able to attach meaning to concepts through significant association (Lynch \& Warner, 2008). Students' multiple intelligences are accounted for in a differentiated classroom. Fogarty and Pete (2005) explained that differentiation is about welcoming each and every learner, in celebration of the differences of each one. Participating in PLCs will allow teachers to build up their instructional repertoire so that they have a better chance of reaching all of their students. There are also a number of societal trends that have led to a greater need for DI. These societal trends include an increasingly diverse student population, high student mobility rates, and federal mandates for inclusive classrooms. These recent trends have contributed to the emergence of SI, an offshoot of DI, which focuses on English Language Development.

\section{The SI Initiative}

Because our nation is more diverse than ever before, SI was developed to help make curricular content comprehensible for English language learners (ELLs). Two of the most prominent models currently used include Guided Language Acquisition Design (GLAD) and Sheltered Instruction Observation Protocol (Echevarria et al., 2010). While SI started out as best practice in teaching ELLs, districts have discovered that SI 
techniques are also effective for teaching all students due to the fact that, at its core, SI looks to differentiate and tap into the talents of each individual learner. An accomplished SI teacher modulates the level of English used with students through techniques such as the use of visual aids, modeling, demonstrations, graphic organizers, front loading vocabulary, adapted texts, cooperative learning, peer tutoring, and native language support. Teachers make specific connections between the content being taught and students' experiences and prior knowledge, and they focus on expanding the students' vocabulary base (Echevarria et al., 2010).

When teachers shelter their instruction, there is a high level of student engagement and interaction with the teacher, other students, and with text, which leads to elaborated discourse and critical thinking. Learning is promoted through social interaction and contextualized communication as teachers guide students to construct meaning and understand complex concepts from text and classroom discourse (Echevarria et al., 2010). Students are explicitly taught functional language skills such as how to negotiate meaning, confirm information, argue, persuade, and disagree. These skills are learned at individual rates, but in a nonthreatening environment where students feel comfortable, confident, and are willing to take risks. The parallels between students learning in a cooperative setting and adults learning in a cooperative setting (in PLCs) are quite evident. Hence, the best way for adults to model best practice for their students is to actively participate in PLCs, and mirror that type of learning environment in their classrooms to facilitate high levels of learning. Moreover, peer coaching, the often overlooked aspect of PLCs, is a powerful way to address differentiated and SI. 
It is ultimately the responsibility of the principal to put teachers and students in the best possible situations to achieve their full potential. This can be done if principals effectively shape and guide the PLC process in their schools. PLCs are the vehicle by which the principles of DI and SI, and teaching and learning in general, can be observed, discussed, and put to use. Whether or not teachers are differentiating and sheltering their instruction can be difficult to quantify. The handbook provides a fidelity checklist that can be used by principals or teachers in peer coaching roles. The data generated from the fidelity checklist can be used in the PLC process to ensure that DI and SI are being used within the school. Some examples of the data PLCs can generate regarding DI and SI include:

- The amount of student-to-student interaction teachers are promoting

- The extent to which teachers are making their classroom a print-rich environment

- The degree to which teachers are promoting literacy and English language development strategies

- Students' knowledge and mastery of both Basic Interpersonal Communication Skills and Content Academic Language Proficiency Additional studies are needed to determine the effectiveness of SI. Most of the research attention for SI has been from schools with populations of students with lower socioeconomic Hispanic backgrounds. There is a need for research on the development of learners from other major ethnic groups in the United States. Students of Vietnamese, Hmong, Cantonese, and Korean backgrounds should be studied because they are the next 
most populous groups of ELL students in the United States (Kindler, 2002). Additional research is also needed on ELLs with impaired capacities of language and academic learning. These students are commonly referred to as dual-identified-students identified as both ELLs and special education students (Genesse, Lindholm-Leary, Saunders, \& Christian, 2009). In order to adequately respond to the changing demographics in our schools, principals must engage PLCs in activities that provide teachers with resources and training for sheltering their classrooms. SI can benefit all students, particularly ELLs, and should be addressed through the PLC process.

\section{The RTI Initiative}

The RTI initiative is closely linked to the PBIS initiative, which will be discussed shortly. Both RTI and PBIS are current school change initiatives that principals cannot ignore. Reviewing the literature on RTI and PBIS is important, as they form the basis for many of the decisions that principals, teachers, and PLCs will make in their schools (Buffum, Mattos, \& Weber, 2009; Prasse, 2009). Some districts are now referring to the combined initiatives as Effective Behavioral and Instructional Support Systems (EBISS). Estacada is currently exploring moving to the EBISS model. RTI is a problem-solving process, whose foundation is the provision of systematic, research-based instruction and interventions for struggling learners. It assumes that instruction is matched to students' needs, and that the monitoring of progress is continuous (Ogonsky, 2008). RTI provides schools with a structure for screening students, providing high-quality instruction, and implementing and assessing targeted interventions within the general education setting. 
Some of the research on RTI suggests that although the Office of Special Education Programs at the US Department of Education funds several projects that support practitioners' use of evidence-based interventions and assessments within an RTI process, there is still much to be completed to ensure that personnel participate in appropriate professional development (L. Danielson, Doolittle, \& Bradley, 2007). Results from various studies of professional development in the 1990s suggested that professional development can influence teachers' classroom practices significantly and lead to improved student achievement (American Educational Research Association, 2005). Professional development has achieved greater importance as the link between practitioner skills and student performance levels has been delineated. To help improve student performance, the critical features of high-quality professional development should be in place, including professional development structures, such as teacher networks and study groups (PLCs) (Kratochwill, Volpiansky, Clements, \& Ball, 2007). In discussions of professional development and building capacity for sustainability, there is an emerging knowledge base present, but again, the research base will need to expand greatly if educators are to be supported in improving achievement of all students-the ultimate goal of the No Child Left Behind Act of 2001 (L. Danielson et al., 2007).

Again, the school's principal is the key leader who establishes how RTI will be managed through the PLC process. Figure 2 highlights the main components of the RTI process. At its core, data drives the decision making. RTI teams establish decision rules to identify students as "core" (at or near grade level), "strategic" (approaching grade level), or "intensive" (significantly below grade level). Universal screening assessments 
are used on all students in a school to determine each student's classification. Once each student's classification is determined, interventions are put into place for each student as part of a multi-level prevention system. Finally, RTI monitors the progress of students in all classifications, with the intensive students being monitored the closest and most frequently (Shinn, Walker, \& Stoner, 2002).

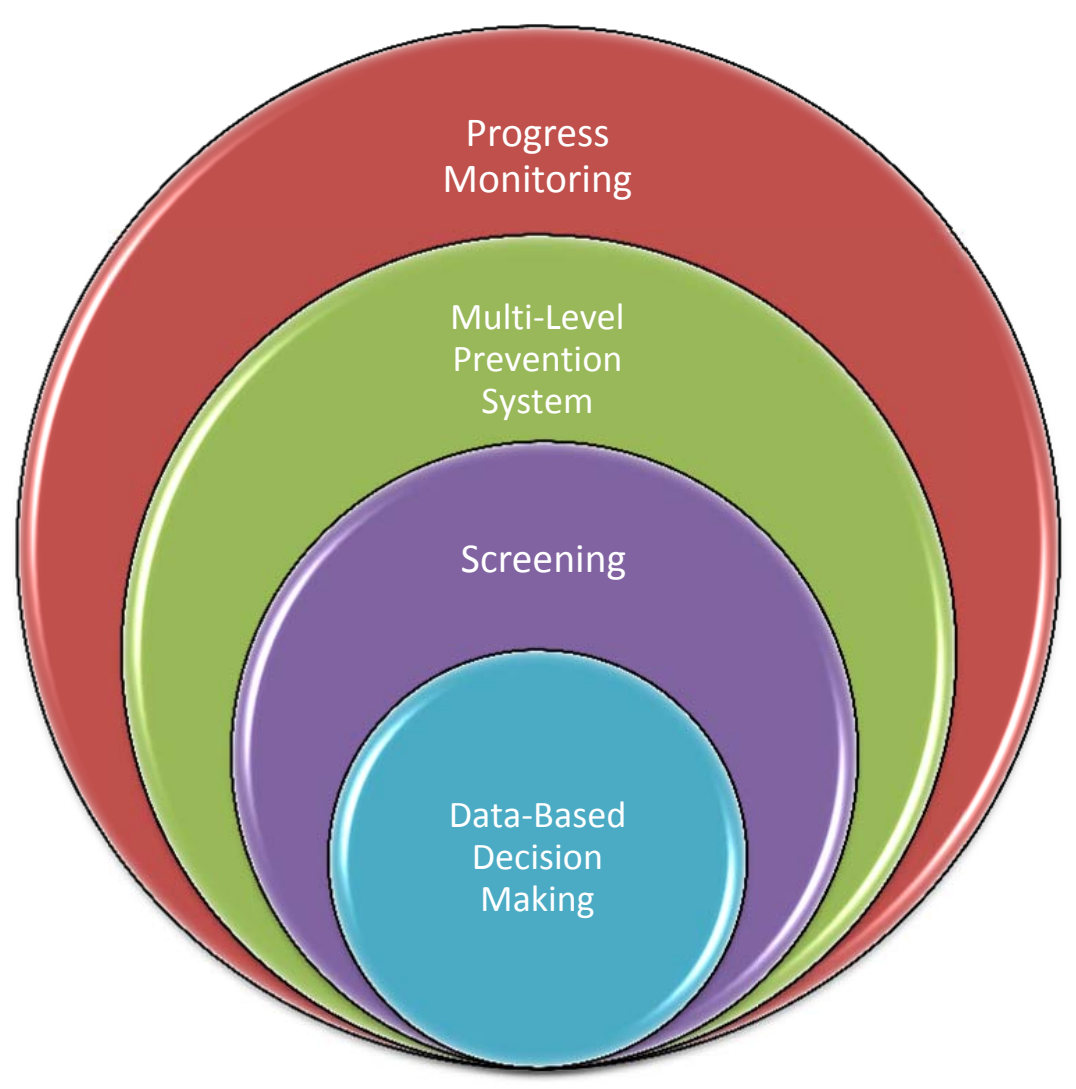

Figure 2. Essential components of RTI. Source: adapted from Shinn, Walker, and Stoner (2002, p. 249).

\section{The PBIS Initiative}

The partner to RTI is PBIS. PBIS is a framework for assisting school personnel in adopting and organizing evidence-based behavioral interventions into an integrated 
continuum that enhances behavioral outcomes for all students (Sailor et al., 2009). PBIS focuses on improving student behavior school-wide through a concerted effort to teach (and re-teach) positive, desirable behaviors. Within every school, there will be "green zone" students-approximately $80 \%$ of the school population-who behave appropriately most of the time. Roughly $15 \%$ of the school's population will be "yellow zone" students. These are students for whom concerns are starting to reveal themselves, but they are not yet an overwhelming problem. Yellow zone students require more targeted behavioral interventions than green zone students. "Red zone" students are the school's "frequent flyers"-roughly $5 \%$ of the school population. Red zone students require the most intense behavioral interventions a school or district has to offer.

Figure 3 illustrates the breakdown of behavioral needs at a typical PBIS school. This model is similar to RTI in that certain groups of students are monitored more closely and more frequently. In the PBIS model, students in the Red Zone are targeted for tertiary prevention methods, which are more specialized and individualized for high-risk behavior students. The progress monitoring of Red Zone students occurs more frequently than for Yellow and Green Zone students, as Red Zone students are considered to be the most at-risk (Crone \& Horner, 2003).

A study of more than 1,000 Illinois schools implementing school-wide PBIS revealed that outcomes were enhanced for students and staff. The sample consisted of the Illinois schools that implemented PBIS from 2000 to 2008 and entered data into the statewide data base. The School-wide Evaluation Tool (Sugai et al., 2001), which is also used in PBIS schools in Oregon, was used as a measuring instrument. Model results were favorable overall, revealing maintenance or improvement in outcomes over time for all 
schools with fidelity to PBIS implementation (Sugai et al., 2010). All schools in the Illinois study sample implemented PBIS, and the percentage of schools implementing it with fidelity increased throughout the study (from $36 \%$ to $78 \%$ ). Schools demonstrated a statistically significant improvement in all social behavior and academic outcome measures (Simonsen et al., 2010). The results suggest why PBIS has become viewed by principals as something that needs to be in place in their schools. While not yet mandated by the federal or state government, PBIS is a highly suggested practice that is implemented in most schools across the country. The handbook will help principals show staff how they can use the PLC process to guide the PBIS initiative in their schools.

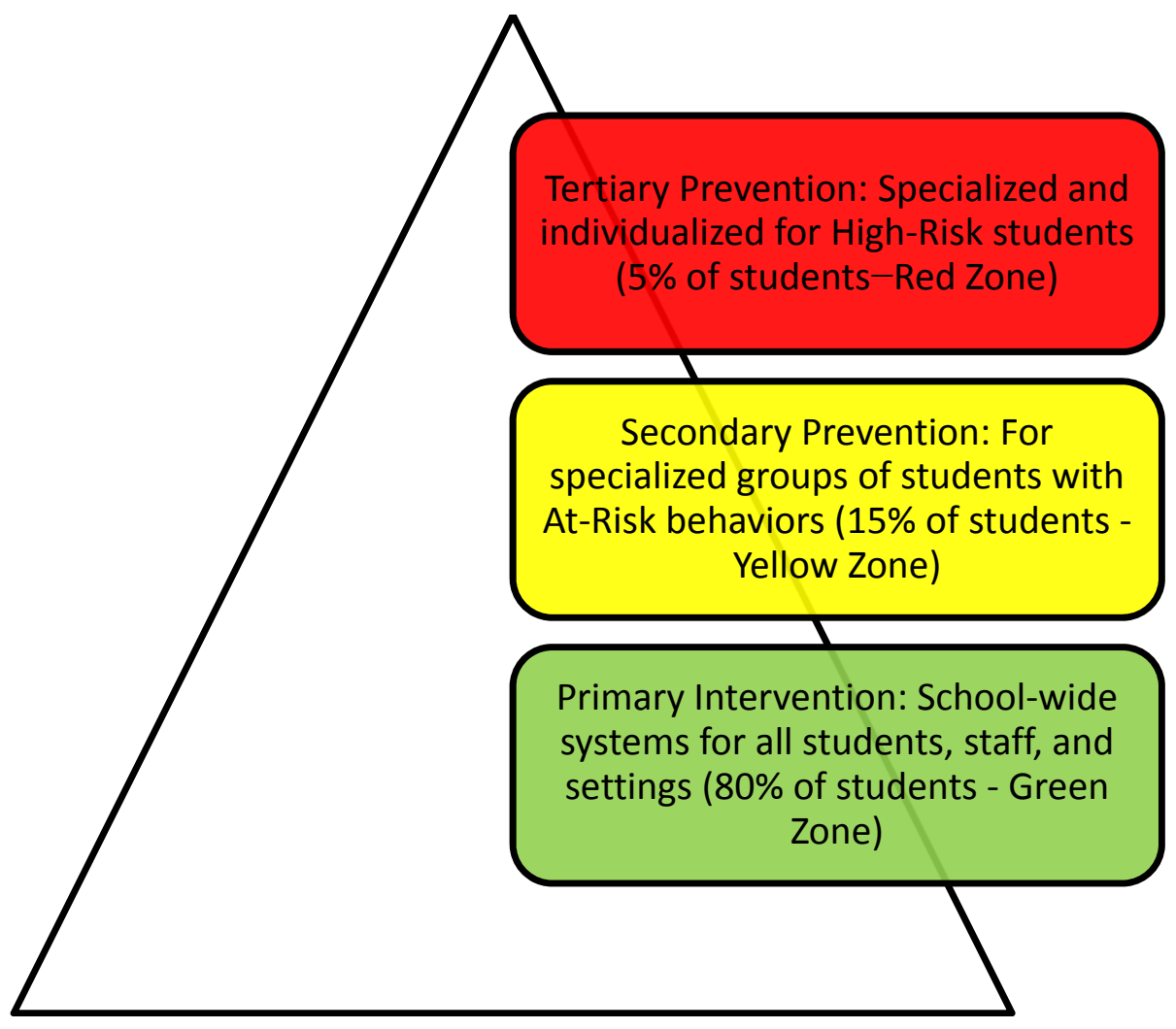

Figure 3. PBIS continuum of school-wide support. Source: adapted from Crone and Horner (2003, p. 19). 
Like with many of the major school change initiatives, there is a great deal of overlap. For example, PBIS is designed to be a school-wide framework. Through use of a school-wide lens, teachers in PLCs are able to look at areas of concern. For example, PLCs can identify information such as the time of day, and the location, where most behavioral infractions are occurring. In addition, PLCs can focus on the type of infractions that are occurring most often; who is committing the infractions; which staff members are reporting the infractions; and what is motivating the students committing the infractions. These pieces of information help the school devise a school-wide plan to remedy the problematic situations. As PLCs zero in on individuals (the yellow and red zone students), the overlap of PBIS and RTI starts to take shape. Individual behavioral interventions are tried with yellow and red zone students, and their progress is monitored. If progress is not made within a reasonable amount of time, then schools go further up their Pyramid of Interventions for behavior (i.e., conducting a Functional Behavior Assessment, implementing a Behavior Intervention Plan, or enrolling students in a Check-In/Check-Out program. If interventions do not yield positive results, it could lead to a referral for special education for behavior. In addition, the data collected could ultimately lead to an educational placement outside of the regular school setting (usually only for "Red Zone" students). Included in the handbook will be a template for a Pyramid of Interventions for both behavior and academics that principals can use with their schools to promote PBIS and RTI.

Like RTI, PBIS requires exceptional organizational leadership by the building principal, and deft use of PLCs. Principals may choose to promote teacher leadership and 
peer coaching in these areas by designating school-wide PBIS and RTI coaches who coordinate the efforts. PBIS and RTI data can, and should, be infused into the PLC process in a school. The handbook will provide helpful graphic organizers that will be useful for principals promoting PBIS and RTI through the PLC process.

\section{The CCSS Initiative}

Reviewing the literature on DI/SI and RTI/PBIS is important as these initiatives drive much of what is taking place in schools today. However, one of the first things a PLC must do is look at what they are going to teach. The CCSS initiative is a state-led effort to establish a common set of expectations across states for what K-12 students are expected to know and be able to do (Council of Chief State School Officers and National Governors Association, 2010). Oregon recently adopted the national CCSS, which are now being phased in under the guise of the ESEA/No Child Left Behind waiver (Oregon Department of Education 2014b). All states will likely soon adopt the CCSS-48 already have. Having national standards across the country will ultimately be a good thing. It will create a more equal playing field when we are able to compare apples to apples (states to states). In the meantime, this type of massive reform effort will make the existence and effectiveness of PLCs more important than ever. It will also increase the pressure for principals to deliver quality leadership and professional development through PLCs to facilitate the transition.

PLC time will be needed for teachers to analyze the new CCSS, and determine what their high priority standards will be so that PLCs can create agreed upon pacing guides and common assessments. Without the PLC time to complete these tasks, teachers 
would not be able to unpack the standards, make them user-friendly, and collect the data necessary to create and apply interventions for struggling students or enrichment opportunities for students who are doing well. Principals will need to provide PLCs with professional development materials and CCSS crosswalk activities. The handbook will provide crosswalk templates that can be used during PLC time. The graphic organizers will help principals and staff transition from the old state standards to the new CCSS.

The adoption process of the CCSS for Oregon included more than the culminating, formal administrative adoption procedure. It also included time for disseminating information about the CCSS to stakeholders and time for gathering feedback and building support before the final, formal adoption procedure (McNeil, 2009). Finn and Petrilli (2010) surveyed education experts to identify key tasks that should be undertaken by practitioners and policymakers to implement the CCSS successfully, and they discovered that collaboration needed to be an imperative part of the process.

Anderson, Harrison, and Lewis (2012) added to the research literature by providing a detailed look at adoption processes in six states for the CCSS and their plans for implementation and assessment alignment from the perspective of directly involved state education agency staff members. Respondents in all six states reported a general process that moved from the development of curriculum and instruction materials to the training of educators to teaching under the new CCSS. One of Anderson et al.'s (2012) main findings was that professional development and collaboration, which are at the heart of PLC work, are needed to make the transition from state standards to CCSS, and to 
ensure a method of prioritization for the new standards (i.e., prioritizing which standards are the most essential).

There are many terms used to describe the most essential standards students should master. Schmoker (2006) has called them power standards. Many districts are now calling them learning targets. Others call them mastery items, and the Oregon Department of Education (2013) is now calling them essential skills (the skills ultimately necessary to graduate high school). Regardless of what you call them, what we are talking about are the most critical skills that students must possess in order to be considered proficient in the recently adopted CCSS. Hence, the review of the literature on standards is especially significant due to the fact that it is an emerging school reform initiative.

Figure 4 illustrates the increased emphasis on reading, writing, speaking, listening, and language across the curriculum under the CCSS, which is a major shift for most districts. For example, in many districts, English teachers have been the main providers of literacy skills for students (SMARTER Balanced Assessment Consortium, 2010). The CCSS make it clear that literacy strategies need to be taught in all subject areas (i.e., science, social studies, health, etc.). Once PLCs have a better understanding of the CCSS their students need to master, they will be in a better position to prepare their students for college and careers beyond high school. It will be important for principals to provide leadership through the PLC process to arrive at a better understanding of how to teach and assess students using the CCSS. Figure 5 illustrates the reorganized framework of the CCSS, with its emphases on college and career readiness and literacy strategies across the various disciplinary areas. 


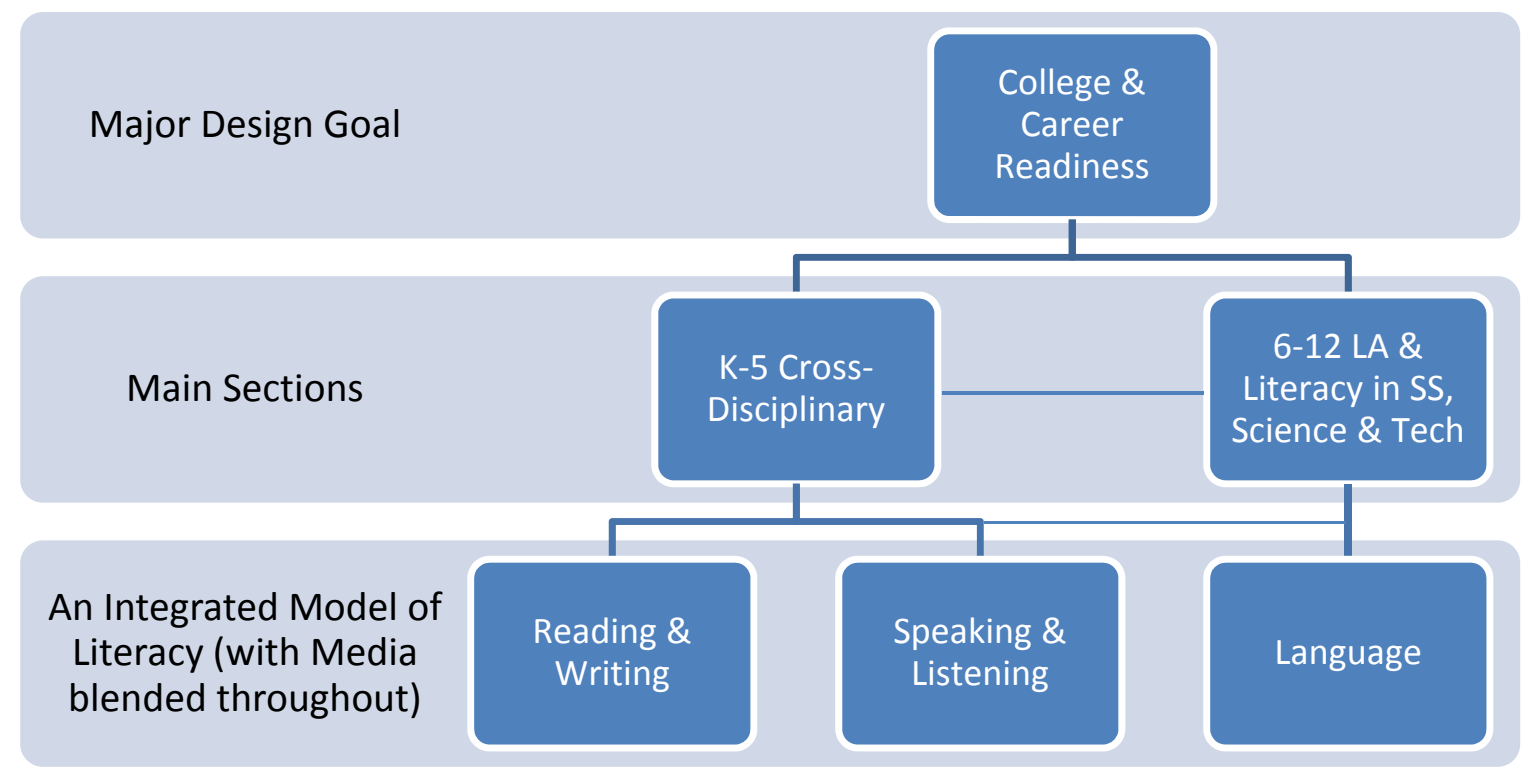

Figure 4. CCSS overview. Source: adapted from Council of Chief State School Officers and National Governors Association Center for Best Practices (2010, pp. 7-9).

\section{The Proficiency-Based Learning Initiative}

Proficiency-Based Learning is another prevalent school change initiative that schools must be addressed in PLC work. The Oregon Department of Education (2013) recently declared that all schools must have a Proficiency-Based grading system (and report card) in place for the 2013-2014 school year. So how do PLCs know if their students are proficient learners? The research indicates that PLCs must agree to give common formative and summative assessments to collect data (Popham, 2008; Stiggins, 2005; Wormeli, 2006). Formative assessments are smaller checkpoints along the way to a larger, more final, summative assessment. Formative assessments are necessary so that PLCs can determine which students will need intervention along the way to the final, summative assessment. Summative assessments are known to be high stakes assessments. 
Summative assessments can take the form of a final unit test, a final project, or even a state benchmark assessment (currently an OAKS test in-soon transitioning to the SMARTER Balanced Assessment, which is linked to the new CCSS; (SMARTER Balanced Assessment Consortium, 2010; Stiggins, 2005).

It is important that teachers do not base a student's entire grade on a small number of high stakes assessments (Kohn, 2000). However, high stakes assessments do not appear to be going away anytime soon. Hence, whatever forms the summative assessments take, there must be an incremental build up, or scaffolding, for the students before they reach that point. Scaffolding, again, is the support given during the learning process which is tailored to the needs of each student as they work to achieve their learning goals (Sawyer, 2006). Scaffolding is critical, and there must be a great deal of checking for understanding along the way (Fisher \& Frey, 2007). It must also be noted that the term "final" summative assessment should be used loosely. Some students will take longer to learn a concept than others, but time should not be the ultimate variable (Popham, 2008; Stiggins, 2005). The definitive goal is that students learn the concept (sooner or later). Proficiency-Based Learning has enormous implications for PLC work, as teams collect and analyze information about student achievement in order to create groupings that allow for extra time and intervention, as well as for enrichment.

Many districts are now moving toward more authentic assessments by using a Proficiency-Based grading system (Meyer, 1992; Wormeli, 2006). This policy change is endorsed by the Oregon Department of Education (2013) and local school boards. What this essentially means is that the state of Oregon and its districts want the grades on report 
cards to be true reflections of what students actually know. Grades should not be inflated because of extra credit or effort. Conversely, students should not be penalized for poor behavior or attendance. Certainly, we want good attendance and good behavior, but these should not be factors in determining a student's grade. Does the student know the concept, and can they demonstrate it? That should be the ultimate question (Wormeli, 2006). If the student does not know the concept, then we as educators need to work harder to intervene so that the student does eventually come to a place of understanding. Along these lines, districts are offering other, less traditional, methods for students to demonstrate their knowledge such as credit by proficiency (project and community service-based), as well as credit recovery programs (which allow for extended learning time). Credit by proficiency refers to students submitting work samples that demonstrate their mastery of essential skills. Credit recovery refers to students taking make-up classes for missed credit through a variety of options such as alternative school settings, online classes, or summer school.

The concept of Assessment FOR Learning, which promotes ongoing teacher feedback, was framed using students' formative assessments, and was spearheaded by Stiggins (2005). Assessment OF Learning is a snapshot of how students perform on one high stakes assessment. Stiggins used the analogy of putting the dipstick into your car to check the oil at one particular moment in time. Assessment FOR Learning-or Proficiency-Based Learning-is an ongoing process where students know learning targets up front, receive continuous feedback from their teacher, and have multiple opportunities to learn content. Stiggins recognized how crucial PLCs are when it comes to assessment 
for learning. He has the belief that collaborative learning teams represent the future of professional development in American schools.

The effectiveness of Proficiency-Based Learning through PLCs has been proven over time by hundreds of successful learning teams in schools across the country (Black \& William, 1998; Stiggins, 2005). In PLCs, teachers are drawn to the promise of time to concentrate on important topics long enough to practice and internalize new and useful ideas. This, combined with time to talk with and learn from colleagues, makes the PLC model of professional development attractive. PLCs need to commit to administering common assessments with their students. They then need to analyze their results, and compare them with their PLC team members. The data they analyze will not only tell them valuable information about their students, it will also become a platform for discussing and sharing teaching strategies with their colleagues so that they can become better instructors (DuFour et al., 2006; Fisher \& Frey, 2007; Popham, 2008, Stiggins, 2005). These practices should be nonnegotiable and will be clearly defined in the handbook to help principals guide the work of PLCs in their school.

Bakula (2010) conducted a research study on Proficiency-Based Learning that involved 95 seventh-grade students. Artifacts, student surveys, journaling, and observations were used to gather data. However, most of the data were gathered from students' six formative assessments and their final summative assessments. Individual student results indicated that when learning targets and rubrics were shared with students at the beginning of the unit, and re-teaching occurred after formative assessments, $95 \%$ of 
the students either improved or stayed the same, and only 5\% showed a decline on the summative assessments.

More evidence on the merits of Proficiency-Based Learning is indicated by Sidhu (2011). Through a range of learning activities, Sidhu planned a sequence of lessons that encouraged students to evaluate and develop their understanding of specific examination topics. Students used teacher feedback from each lesson to inform and refine their learning. Student focus groups revealed the extensive impact that this approach had on the students' own motivation for learning. In addition, staff observed and reported a $100 \%$ increase in student motivation. From this, we can see that assessment for learning activities helped increase student engagement. There was also a noticeable increase in how well-prepared students were during the course of the 2-week period they were observed. Furthermore, the summative assessment results showed a strong positive relationship between student-focused assessment for learning and increases in student achievement. All students included in the study improved their assessment scores, and $60 \%$ of the students exceeded their target grade.

From his vast meta-analyses of more than 75 sources on instruction and assessment, I. Clark (2011) noted that when feedback is used effectively, it is the most powerful single motivator that enhances student achievement. Proficiency-Based Learning is an instructional approach that closes the gap between students' current understanding and the desired learning goals. Figure 5 presents the key questions that must be asked by educators intending to promote Proficiency-Based Learning in their classroom. 


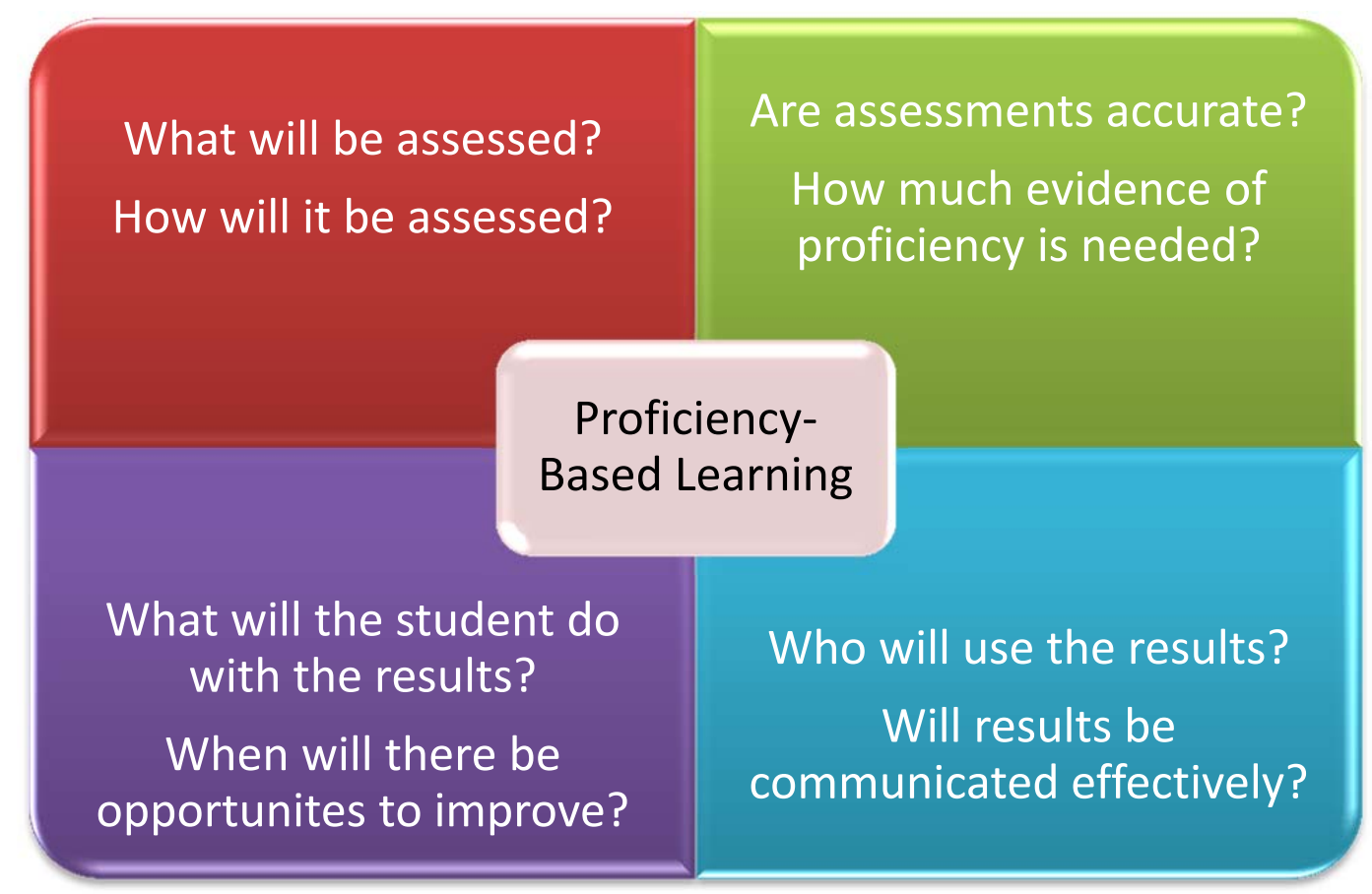

Figure 5. Proficiency-Based Learning: Key questions of the assessment for learning process. Source: adapted from Stiggins (2005, p. 33).

As indicated in Figure 5, PLCs must first identify the learning targets (or priority standards). They must then determine how the learning target will be assessed (project, essay, presentation, etc.), and provide student-friendly rubrics at the beginning of the units and lessons. Next, PLCs must ask if the assessment is an accurate measure of learning, and determine how much evidence is needed to declare that students are proficient. Once the results from the assessment have been collected, PLCs must determine who will use the results (ideally all stakeholders-teachers, students, and parents). PLCs must also determine how the results will be effectively communicated. Finally, it is important for PLCs to indicate what the students will do with the results, and determine when opportunities for improvement will be granted. Proficiency-Based 
Learning is not time bound-its relationship to the mastery of essential skills by students is what is critical. Through PLCs, teachers can monitor how and when their students achieve proficiency of essential skills.

\section{Summary of the Initiatives}

The school change initiatives discussed in this literature review all require new ways of thinking about teaching and learning. They also require moving away from outdated past practices, such as working in isolation. Collaboration among staff members needs to be strong to meet all of the demands that these initiatives require. PLCs, guided by the principal, should be effective in implementing new solutions to the emerging demands placed before them. There are certainly areas that overlap across the school change initiatives, and PLCs are the natural vehicle to move the initiatives forward. The handbook for PLC leadership will help principals show staff how working in PLCs can help them address important change initiatives in their schools.

\section{Principal Leadership}

In the review of the literature regarding principal leadership, I will use a conceptual framework from Matthews and Crow's (2010) research that references the multiple roles principals must fulfill to create conditions in which PLCs can be effective. Much of the research (Bogdan \& Biklen, 2007; Thompson et al., 2004) on principal leadership used mixed methods design, which is significant because it provides both statistical data as well as perceptions from the field. Mixed methods research designs are procedures for collecting both quantitative and qualitative data in a single study, and for analyzing and reporting the data based on priority and sequence of the information 
(Creswell, 2005). A fair amount of the quantitative information was collected from large groups, and it was analyzed statistically. Qualitative methods collected information from principal and teacher focus groups, highlighting the importance of inquiry in the PLC process. Most of the qualitative data were focused on the interactions of PLCs. Studies showed that principals who supported the creation of a collaborative work culture through PLCs adopted a more democratic leadership style where everyone is a learner and leader. Information relevant to group dynamics and human interaction has been used by principals to gain a better understanding of PLCs and their effect on school culture (Bogdan \& Biklen, 2007; Thompson et al., 2004).

While the classroom teacher has the most important role in influencing student learning on an individual basis (Marzano et al., 2005), no single individual is more important to initiating and sustaining improvement than the school principal (Jackson \& Davis, 2000). Through PLCs, the principal can bring diverse groups of people together who have shared interests, and can expand leadership throughout the school (Thompson et al., 2004). Teachers in studies that date back to the early 1990s have identified the supportive leadership of their principal as one of the necessary human resources for schools to become effective in using collaborative teamwork models (Louis \& Kruse, 1995). In terms of principal leadership, major factors necessary to successfully facilitate collaboration in a school include the principal's ability to share authority, to facilitate the work of the staff, and to participate without dominating (Prestine, 1993). Principal leadership is crucial to PLC work because the principal helps teachers develop trust in the PLC process. 


\section{The Role of the Principal in PLCs}

In the 1970s, a line of inquiry known as "effective schools research" sought to explore the school conditions under which students performed better than their socioeconomic background might have predicted. Though this research was questioned on theoretical and methodological grounds, its conclusions were taken seriously as providing guides for school improvement (Edmonds, 1986). One of the core factors identified as contributing to school effectiveness was strong leadership, enacted through principals' high expectations for student achievement, high support for staff, and strong goal and task orientations (Rosenholtz, 1985). All of these attributes speak to the principal's role of guiding the work of PLCs.

One of the first things a principal should do is work to create a shared vision with his staff (Riehl, 2000). As principals work with staff to create a shared vision, the research behind PLCs should be provided in order to help generate buy-in. The current studies that formed the basis of my analysis of PLCs are mainly qualitative case studies, although some of them added quantitative data in the form of survey results or students' standardized test results. Most of the qualitative data reported in these studies were from interviews, observations, field notes, and meeting transcriptions that were then reported in a case study format. Qualitative case studies, in particular, are significant when it comes to the role of the principal and PLCs because they reveal findings, reflections, and recommendations from working practitioners in the field. Studies that were reviewed offered empirical data demonstrating that keeping the focus on student learning should be the principal's main objective (Vescio et al., 2006). 
The studies reviewed also cited data suggesting that a change in the professional culture of a school takes place when operating in PLCs. Culture played a key role, and the principal was the one who established the expectations for PLCs. Based on the work of Senge (1990) and Senge et al. (2000), the features of PLCs, in terms of systems thinking, are critical in facilitating collaboration from a management standpoint. Establishing a systematic approach to PLCs improves the functioning of group members and the organization as a whole. Scribner et al. (1999) highlighted this important implication. The need to understand the factors and characteristics that define a school's place on the PLC continuum is significant. For example, the most important facilitating or impeding factor discussed in their research was the role of the principal. Although all principals felt the utmost respect for their faculty, and were all concerned with student well-being and achievement, their leadership styles played critical roles in the degree to which PLCs operated. This case study gives us a better understanding of antecedent conditions, as well as intervening actions that lay the groundwork for establishing PLCs. Principals can create the conditions conducive to effective PLC formation and operation by:

- Leading staff development activities that promote collaborative work

- Facilitating meetings that focus on data and student learning

- Providing leadership opportunities for teachers

- Providing forms and graphic organizers that lead to future action

It is important to understand that the PLC process is ongoing, involving different people, tasks, times, places, and ideas. Figure 6 highlights the critical components of PLC work. Time and again the research points to these components as being essential for PLC 
effectiveness. Principals must ensure that PLCs are adequately addressing these major components, starting with identifying essential learning standards.

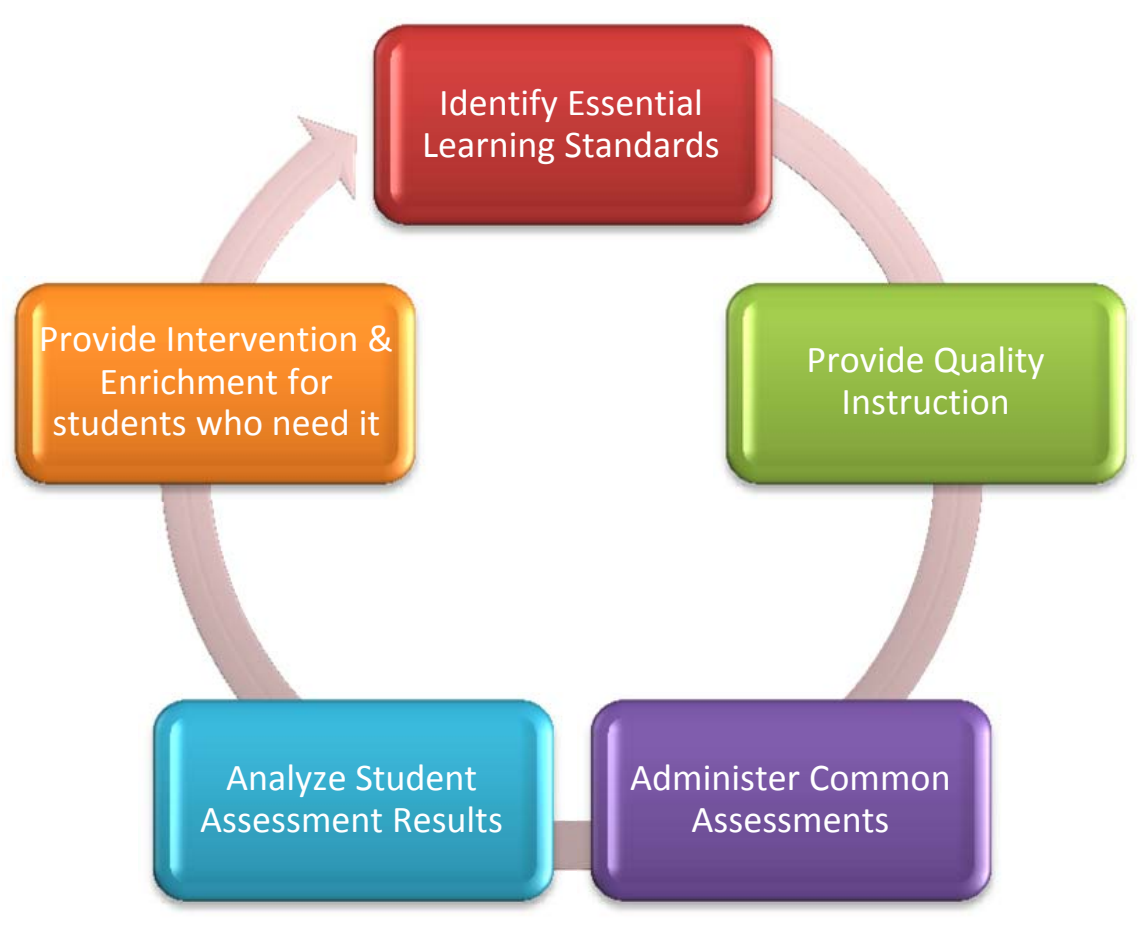

Figure 6. The PLC cycle. Source: adapted from DuFour et al. (2006, p. 121).

PLCs are effective when teachers and principals work together, share their knowledge, contribute ideas, and develop plans for the purpose of achieving educational goals. Collaboration through PLCs is demonstrated when school staff members come together on a regular basis in their continuing attempts to be more effective teachers so that students can become more successful learners (Leonard \& Leonard, 2001). Principals need to develop teachers who exhibit leadership skills. Teachers exerting leadership will be committed to action, and will be agents of change as they work to meet the challenges facing educators today. Senge (1990) discussed the impetus for change often coming first 
from the principal, who should serve as a lead teacher, learner, and steward of the learning process.

Figure 7 highlights the various roles of the principal (throughout the school, not just in PLCs). This figure shows that principals are the leaders for schools in a variety of areas that often pull them in multiple directions. This type of challenge demonstrates that principals must rely on teacher leadership through PLCs in order to respond to a multitude of areas of concern and interest.

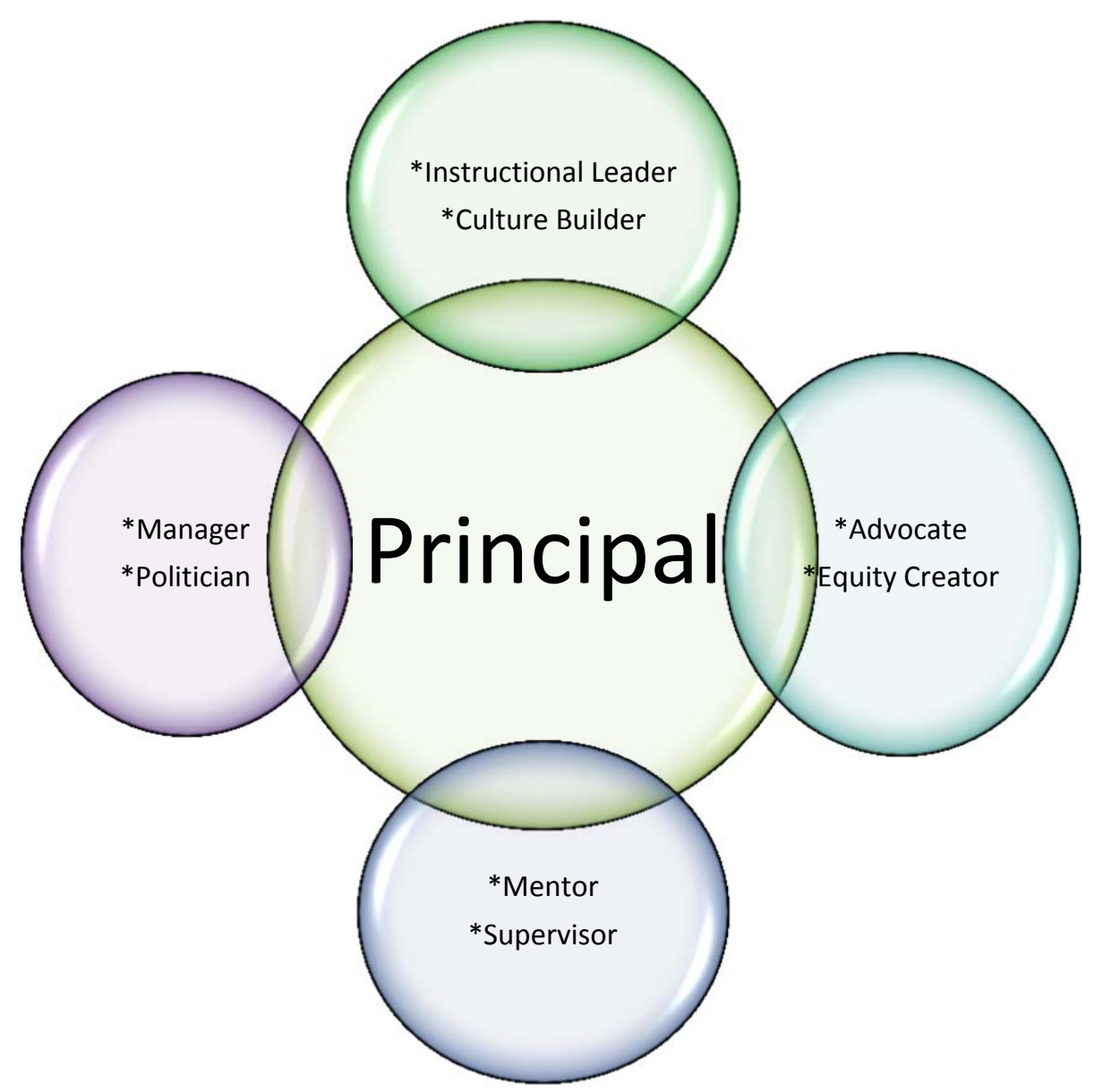

Figure 7. The various roles of the principal. Source: adapted from Matthews and Crow (2010, pp. 13-16). 


\section{The Principal as Learning Leader and Culture Builder}

As the steward of the learning process, the principal has a particular responsibility to build a positive culture and lead the staff in developing a shared vision for learning. Sergiovanni (1995) stated that a shared vision should not be construed as a strategic plan that functions like a map charting the turns needed to reach a specific reality that the leader has in mind. The vision should be viewed as something that points the direction to be taken and inspires the school as it moves forward. Enthusiasm for learning allows educators to buy into, and take part in, the shaping of the school's vision, leading to a bonding of principals with their staff. Nanus (1992) suggested that to promote a positive school culture focused on student learning, the principal should be a direction-setter, a change agent, a spokesperson, and a coach. The principal is responsible for catalyzing changes in the environment to make the vision achievable. Principals can build on what teacher leaders determine as needs, and can use the PLC process as a vehicle for developing and enriching student learning experiences.

Being an effective instructional leader and culture builder equates to being a transformational leader. According to Northouse (2001), in the simplest terms, transformational leadership is the ability to get people to want to change, improve, and be led. It involves assessing staff members' motives, satisfying their needs, and valuing them. Some researchers claim that transformational leadership is the leader's ability to increase organizational members' commitment, capacity, and engagement in meeting goals (Chew \& Chan, 2008; Geijsel, Sleegers, Stoel, \& Kruger, 2009; Leithwood \& Jantzi, 2006). These ideas around transformational leadership help describe the 
principal's role in the PLC process. Transformational leadership has three basic functions. First, it serves the needs of others and empowers them to achieve their goals. Secondly, it instills trust, confidence, and pride in staff members. Finally, the school becomes empowered as a collective unit (Castanheira \& Costa, 2011). Transformational leaders enhance motivation, morale, and the performance of followers through a variety of methods, including:

- Connecting the follower's sense of identity and self to the PLC process and the collective identity of the organization

- Being a role model for followers that inspires them and makes them interested

- Challenging followers to take greater ownership for their work

- Understanding the strengths and weaknesses of followers, so the leader can align followers with tasks that enhance their performance

Balyer (2012) conducted a study focusing on 30 teachers from six different schools. The participants were chosen by using a sampling method, with the intent of understanding human perceptions, problems, needs, behaviors, and contexts. The data were collected using the repertory grid technique, which is a constructed interview method. The technique can be best characterized as a semi-structured interview (face-toface, computerized, or by phone) in which the respondent is confronted with a triad of elements and then asked to specify some important ways in which two of the elements are alike, and thereby, different from the third. The findings from this study indicated that the principal is undoubtedly the key instructional leader and culture builder of a school. The leadership behaviors of the principal have significant direct and indirect influences 
on teachers' commitment to change, as well as their overall performance. As learned from the study, teachers want their principal to set high expectations and motivate them in achieving their goals. Teachers in the study also indicated that their principals make teamwork possible at the school by providing opportunities for staff to collaborate. High expectations and teamwork are essential elements of effective PLCs, and these elements highlight the need for strong leadership from the building principal.

\section{The Principal as Mentor and Supervisor}

In order for PLCs to have their desired effect, principals must be able to effectively mentor and supervise teachers. Cultivating teacher leadership is a critical component. Principals must guide their staff in an effort to capitalize on their talents as

well as improve their weaknesses. When considering the PLC process, principals need to understand the individual strengths and needs of each teacher (Johnson \& Kardos, 2002). The principal must also be able to identify key teacher leaders who demonstrate exemplary characteristics that are congruent with current measures of educator effectiveness, such as:

- Demonstrating knowledge of content and pedagogy

- Creating an environment of respect and rapport

- Communicating with students and providing feedback

- Reflecting on teaching experiences

It is important for principals to nurture teachers who are (or can be) leaders (C. Danielson, 2006; Elmore \& Wisenbaker, 2000). From their research on school principals mentoring and supervising staff, Lambert et al. (2002) have the belief that guidance by 
the principal is more important than ever in this age of accountability where collective action must be taken to change the learning experiences for both students and teachers. School leaders should possess a set of beliefs, skills, and knowledge about leadership that emphasizes reciprocity and sharing among the adults of the building. Reciprocity refers to the back-and-forth communication that takes place among school community stakeholders who work in PLCs. Reciprocity and sharing are cornerstones of collaboration, as purposes and goals grow among participants based on values and beliefs as well as individual and shared experiences (Lambert et al., 1995).

While the role of principal is central to the success of a school, the idea of one person being able to create successful PLCs is outdated (Matthews \& Crow, 2010; Thompson et al., 2004). Fullan (2009) stated that principals can make even more longlasting contributions by broadening the base of leadership of those with whom they work-teachers, parents, and students. As educators become more and more convinced of the benefits to be derived from a school that promotes PLCs, principals will be able to encourage teacher leadership more effectively. Principals should set school conditions that promote the emergence of teacher leaders (C. Danielson, 2006). For example, it is recommended that principals identify at least one main teacher leader or coach for each prevalent school change initiative in order to help move efforts forward.

Encouraging teacher leadership will not only empower teachers, it will also help alleviate the problem of the school principal being spread too thin. In addition, teacher leadership will strengthen the possibility for peer coaching in the PLC process, and encourage a culture of instructional risk-taking, which will ultimately lead to better 
overall teaching throughout the school. Principals should convey to all staff that the teaching environment is safe, and teachers should be encouraged to take instructional risks. Allowing for instructional risks suggests that there are no penalties for mistakes, and that the lack of complete success is valued because it provides insights into what is not yet working (Hoerr, 2005). This is why establishing lead teachers for peer coaching is so desirable. Principals can provide professional development opportunities through the peer coaching aspect of PLCs. This is important because, while still a mentor, the principal also needs to remain the supervisor of teachers-the person who completes formal evaluations. In the supervisor role, the principal will sometimes need to address personnel issues that could lead to disciplinary action, formal plans of assistance, and sometimes dismissal of teachers.

Calik, Sezgin, Kavgaci, and Kilinc (2012) conducted a study that examined the relationships between school principals' instructional leadership behaviors and teachers' self-efficacy and collective efficacy. Self-efficacy is defined as people's beliefs about their talents to activate motivation, cognitive resources, and actions needed for ensuring control over the events in their lives. Collective efficacy is a group's shared belief about actions needed to achieve outcomes (Wood \& Bandura, 1989). This research sample consisted of 328 classroom teachers. The Teachers' Sense of Efficacy Scale, developed by Tschannen-Moran and Hoy (2001) was used to measure teachers' self-efficacy, and to gather data about teacher collective efficacy. In addition, the Collective Efficacy Scale developed by Goddard, Hoy, and Hoy (2000) was used. To evaluate school principals, an instructional leadership scale developed by Sisman (2004) was used. The study revealed 
that instructional leadership had a strong relationship with collective teacher efficacy when compared to teachers' self-efficacy. Instructional leadership provided by the principal through PLCs was an effective way to help teachers feel more confident as they worked to grow professionally and strive to meet student needs. As a result, it can also be asserted that the self-efficacy of teachers will increase when they work in teams (PLCs) guided by strong instructional leadership from their principal.

\section{The Principal as Advocate and Equity Creator}

The principal must also fill the roles of advocate and equity creator, which have important implications for PLCs. Once the PLC process is established, the principal will begin to rely more heavily on teacher leaders for needed information. For example, data regarding student achievement will be presented to the principal by PLCs and lead teachers or coaches in the building. The principal will need to know what can be done do to support each PLC in terms of supplies, resources, and materials. It will be up to the principal to ensure that there is equity regarding the type of support provided among all PLC groups. In addition, individual priorities must be evaluated within the global view of the school's priorities (Matthews \& Crow, 2010). Depending on the resources available, the principal may also encourage teacher leaders and PLC members to write grants for the supplies, resources, and materials they are seeking.

As an advocate and equity creator, questions must be asked on a regular basis in order to challenge assumptions. For example: How will learning be measured? How can students be organized for optimal learning? What instructional processes are available? How do we accommodate for individual differences? What are the implications of our 
instructional processes on the other organizational components of the school? How can our processes be refined and improved? Undoubtedly, educators accept that every student learns at their own individual rate, and the principal must ensure that equal access to education is granted. Some examples of areas where equity must be constantly examined include access to programs, curriculum, tools, and technology (Garland, 2010). The examination of these areas can take place through the PLC process, with the principal being the key leader.

Factors such as special needs, language levels, abilities, interests, learning styles, and personalities are difficult to assess, for they are complex and varied, but they all require advocacy (Ubben et al., 2004). Educators must strive to diagnose these factors through PLCs to provide the most equitable learning environment possible. Principals must ensure that teachers know their students' strengths and weaknesses. As educators, we need to establish the best possible conditions so that all students, regardless of background, have an equal opportunity to achieve high levels of academic success. Because of this, the principal must ensure that PLCs are collecting and analyzing student data on a regular basis so that the school can make informed decisions about how to best serve each student (Stiggins \& DuFour, 2009).

In addition to supplies, resources, and materials, time is also important. The principal must protect PLC time, and guarantee that other competing interests, outside of the school's shared vision, do not start to encroach on PLC time. Fullan (2009) has suggested that many professional development strategies have been fragmented and are oblivious to the needs of teachers. Many professional development activities take the 
form of something being done to teachers, rather than something being done with them. Once again, this view highlights the great potential for the PLC process (including the peer coaching model). The principal, using the PLC process effectively, can promote an equalized learning environment. The principal can also address current school change initiatives, and encourage shared leadership among the staff. Finally, PLCs keep important documentation that will promote equity and advocate for students' learning needs. The handbook will provide templates for important documentation that PLCs should keep (i.e., weekly minutes, task sheets, and academic and behavior checks).

As an educational system, we are improving in terms of promoting equity for all students. This is due, in large part, to educational leaders such as building principals advocating for social justice. Social Justice is a process, not an outcome, which: (a) seeks fair (re)distribution of resources, opportunities, and responsibilities; (b) challenges the roots of oppression and injustice; (c) empowers all people to exercise self-determination and realize their full potential; and (d) builds social solidarity and community capacity for collaborative action (University of California Berkeley, 2013). PLCs are helping schools push the social justice agenda forward. However, we still have a long way to go. While there is still work to be done, an example of the recent progress made can be seen in a study conducted by Posselt, Jaquette, Bielby, and Bastedo (2012). The data for this study consisted of a nationally representative sample of high school completers from the 1972, 1982, 1992, and 2004 high school senior classes, utilizing the National Longitudinal Surveys from those years. The study revealed that the percentage of each racial/ethnic cohort enrolling in postsecondary education within 18 months of graduation 
has increased. For example, the percentage of Black students enrolling in postsecondary education increased from $26.3 \%$ to $52.6 \%$ from 1972 to 2004 . Principals must continue to provide leadership and encourage these upward trends. Professional development activities that advocate for equitable educational practices can take place, and can be refined, through PLCs.

\section{The Principal as Politician and Manager}

In this era of increased accountability, documenting PLC work is a necessary part of the process. Documentation that is collected through the PLC process can help principals and staff members reflect on past practices and inform future practices (Pollock, 2007). Many mandates come to principals from politicians in state or federal government. In order to remain in compliance with these mandates, principals need to provide politicians with evidence and data. Successful school-based data initiatives are almost always influenced by principals who are employing practices such as setting clear expectations for data use, involving entire faculties, and making time for teachers to study their data (Datnow, Park, \& Wohlsteter, 2007; Halverson, Prichett, \& Watson, 2007).

For an examination of district-wide effects on data use, Wayman, Cho, Jimerson and Spikes (2012) focused their research on three school districts in Texas during the 2009-2010 school year. They employed mixed-methods in conducting their study. Phone and in-person interviews were conducted with individuals, site visits were made to schools to conduct educator focus groups, and a confidential online survey was made available to all educators in each district. The results of the study highlighted the roles of 
politician and manager for the school principal, as attitudes toward data were analyzed. Principals in the study described using various data to gauge the fidelity of curriculum implementation. They also ranked the use of data for identifying the needs of struggling students and for developing recommendations for intervention as the top two most frequent uses of data. Using data effectively through the PLC process will allow principals to satisfy political mandates, and also manage their staff as they collectively strive toward high levels of student achievement. From this study of Texas school districts, qualitative data showed that faculty members' struggles with data use were often connected to the leadership of their principals. The highest rated principals in the study had established structures that promoted regular, consistent data use in their schools. Furthermore, these highly rated principals were particularly active in developing robust collaborative routines. Not only did they support teacher-to-teacher collaboration, they also worked directly with teachers on data-related activities and used a collaborative, collegial style in setting expectations and plans using data.

When filling the roles of politician and manager, principals must also be astute at managing risk. Risk, in this sense, is defined as threats the principal must protect the school from, and should not be confused with the principal providing an educational environment that encourages instructional "risk-taking" by teachers. Teachers should, in fact, be encouraged to take instructional risks, but the term "risk" has multiple meanings and interpretations for principals, which are constantly expanding and becoming more complex (Cleary \& Malleret, 2007). Risk, for principals, involves social, cultural, ethical, political, legal, psychological, economic, environmental, and technological elements 
(Adams, 2009). Given the rising prominence of risk, it is little wonder that risk management now plays a major part in schools where children and their futures are concerned. Potential hazards and their consequences interact with a myriad of social, cultural, psychological, and educational processes that accentuate risk. Increasing litigation claims against education authorities impel governmental interest in the oversight and control of risk, with principals mandated to take preventative measures to avoid risk (Apgar, 2006). Responsible school principals must develop risk intelligencethe ability to classify, characterize, store, retrieve, and act upon relevant information. Principals need to be able to communicate risk and risk processes effectively, and adjust risk practices to changing circumstances. Principals have a duty to control risk as far as they can, even though there is little evidence that organizational effectiveness is improved as a result (Leithwood, 2007).

One recent study that was able to collect data regarding risk management by principals was conducted by Starr (2012). The data emerged from a 3-year study into the learning requirements of school principals. Both newly appointed and experienced principals were interviewed to explore perceptions about the essential learning required to successfully conduct the role (assuming that inexperienced principals would be able to recollect recent steep learning curves encountered, while experienced principals would recount wisdom from years of experience). Data collection occurred through intensive, semi-structured interviews (conducted face-to-face and via telephone) with 100 principals, and through discussions recorded as field notes. Principals in the study unanimously believed that risk compliance absorbed escalating amounts of energy and 
time, with mandatory requirements intruding on the core business of teaching and learning. Making a difference in students' lives is not easy and it is often difficult to take seemingly helpful actions without fear of contradicting some unrelated policy, law, or unwritten expectation (Perry, 2007). In other words, making hard leadership decisions about school improvement can actually incur enormous professional risks for principals whose work contracts are in the hands of others who may be motivated by personal rather than organizational interests. Principals could reduce personal risk if they make time for collective engagement through PLCs to exert more influence and control over policy, procedure, and direction in education for the benefit of themselves, students, teachers, and the community in general (Bottery, 2003).

\section{Summary of Principal Leadership}

The work of a school principal is complex and multifaceted. Principals are the key leaders in a variety of roles within the school and district community. The principal is the instructional leader and culture builder of the school. The principal is also the mentor and supervisor of teachers. In addition, principals act as advocates and equity creators.

Finally, school principals must serve as the school's main politician and manager. These numerous and varied roles accentuate the principal's need to promote shared leadership and PLCs in their schools. PLCs can be a way to promote learning and culture-building activities. PLCs can also provide professional development opportunities where teachers learn from one another. In addition, PLCs can provide an environment that promotes equity for students and teachers and can ensure that students' individual needs will be advocated for, not overlooked. Moreover, PLCs can provide the required documentation 
that principals and schools need to satisfy state and federal mandates that require evidence and data.

\section{Summary}

The research clearly states that implementing PLCs in a school is best practice, meaning that collaboration and teamwork will always prevail over teachers working in isolation. There are several documented benefits to having PLCs serve as the vehicle for school improvement. PLC members benefit from sharing their successes and failures. PLCs share ideas and techniques for what they believe will benefit the highest number of students in the school. PLCs agree on the most essential learning targets-the priority standards-that they will focus on with students. PLCs inform their decisions about future intervention or enrichment opportunities based on data generated from common assessments. Schools can use PLCs to address important school change initiatives (DI/SI, RTI/PBIS, CCSS, and Proficiency-Based Learning). In addition, PLCs have enormous implications for principal leadership. PLCs allow for a more democratic style of leadership because input and feedback is constantly being solicited from a wide variety of school community stakeholders. PLCs also help principals build capacity for teacher leadership, which enables the principal to fill the numerous various roles that come with being the key leader of a school. 


\section{CHAPTER 3}

\section{METHODOLOGY AND RESEARCH TECHNIQUES Introduction}

The research framework used for this study is grounded in PBL (Bridges \& Hallinger, 1995). The PBL design takes the findings generated by the basic and applied R\&D process and uses them to build and refine tested products that are ready for operational use in schools (Borg \& Gall, 1989). Data were collected while developing, field-testing, and refining a handbook for PLC leadership. The data were coded, and key patterns and themes were highlighted (Creswell, 2005). Data that were gathered and analyzed related to the effectiveness of the product (the handbook)-Guiding the Work of Professional Learning Communities: Perspectives for School Leaders (see Appendix). Four principals and one vice principal from the Estacada School District served as a design team. As early as the 1970s, Hackman (1976), through his work studying the guidelines for structuring, supporting, and managing groups in contemporary organizations, identified design teams as having three major classes of tasks: (a) Production tasks, (b) Idea-generation tasks, and (c) Problem-solving tasks. Production tasks include actually making something, such as a product. Idea-generation tasks deal with creative tasks, such as brainstorming a new direction or creating a new process. Finally, problem-solving tasks refer to determining plans for action. The design team helped create, field-test, and refine the handbook for PLC leadership. 
After the design team field-tested the handbook in their schools, their role shifted from being a design team member to a more traditional focus group member. A focus group member is a person who is asked about their perceptions, opinions, beliefs, and attitudes toward a product, service, or idea. Questions were asked in an interactive group setting where participants were free to talk with other group members (Kaufman, 2003). The data for this study was collected primarily through structured interviews using the Critical Friends Consultancy Protocol (Harmony Education Center, 2013). The structured interviews established by the researcher consisted of collecting data through interviews with a group of people, five in this case (Creswell, 2005). There was no personal risk for the design team members, as this study looked to assess and evaluate the effectiveness of the product (the handbook).

The design team collected data and shared their understanding about the handbook activities that were most helpful for principals in guiding the work of PLCs in their schools. In addition, the study looked at how principals could use the PLC process to address major school change initiatives (DI/SI, RTI/PBIS, CCSS, and ProficiencyBased Learning). The design team assisted the main author of the handbook with the development and refinement of PLC activities, and provided valuable insights from their experiences field-testing the handbook in their schools. To supplement the research from the design team approach, team members also reviewed documents provided by their teachers, including weekly PLC minutes. Other data sources included the review of institutional documentation, structured interviews with teacher leaders, and survey results. 


\section{Brief Restatement of Problem}

PLCs are crucial to helping schools meet the needs of students and address school change initiatives, but they are difficult to implement effectively (DuFour et al., 2006). PLCs take place at the same time in multiple settings, and a principal cannot be in multiple places at one time. However, the problem is much deeper than that. Other challenges principals might face when implementing PLCs include:

- Poor group dynamics within PLCs

- Weak teacher leadership within PLCs

- A lack of focus on intended PLC work

- A lack of understanding of current school change initiatives (discussed in detail in chapter 2)

It is important for a principal to understand group dynamics, as well as the tasks each PLC is working on in order to guide future efforts. It is also important for principals to have tools to help PLCs work through challenges that will inevitably occur throughout the course of a school year. In addition, principals must ensure that teachers understand, and help to address, multiple school change initiatives. Principals can use the PLC process as the vehicle to help make these multiple school change initiatives manageable. The handbook was intended to provide the necessary materials for principals to facilitate and guide an effective PLC process in their school.

\section{Research Design}

The research design is theoretically and practically grounded in PBL (Bridges \& Hallinger, 1995) and in the R\&D process (Borg \& Gall, 1989) because the design team 
identified a problem and created, field-tested, and refined a product that helped address the problem. The problem is that while PLCs are widely accepted, they are difficult to implement and sustain and the role of the principal is ambiguous. The handbook defines the role of the principal in the PLC process, and also provides guidance for PLC leadership.

Borg and Gall (1989) identified 10 key steps in the R\&D process: (a) Research and information collecting; (b) Planning objectives, learning activities, and small-scale testing; (c) Developing preliminary form of the product; (d) Preliminary field-testing; (e) Main product revision; (f) Main field-testing; (g) Operational product revision; (h) Operational field testing; (i) Final product revision; and (j) Dissemination and implementation. For the purposes of this dissertation, steps 1-7 of the R\&D process were followed.

1. Research and information collecting attempts to answer this question: Does the proposed product meet an important educational need? Yes, it does. The design team has observed and studied a number of schools that have implemented PLCs. PLCs are a widely accepted practice, but there are numerous challenges. Implementing PLCs is an overwhelming and complex process. Furthermore, the role of the principal has not been clearly defined, nor have resources been provided for facilitating principal leadership of PLCs. Principals and schools are trying to grapple with the complex issues involved with implementing major school change initiatives to meet state and federal measures of accountability (DI/SI, RTI/PBIS, CCSS, and Proficiency-Based 
Learning). Schools struggle to find time to collaborate to sufficiently address these initiatives. The design team believes that PLCs could be the vehicle for implementing these initiatives. Moreover, principals need tools to help manage all of the potential pitfalls that come along with group work and human dynamics. For this step, the design team demonstrated that a handbook for guiding the work of PLCs met the perceived needs of school principals by providing helpful information, activities, tools, visuals, and graphic organizers that could be used with staff. The design team collected and reviewed PLC handbooks from other school districts. I shared my literature review with the team, along with a prototype of the PLC handbook for our district. Based on feedback from the design team, the organizational structure and sections of the handbook were refined.

2. Planning objectives, learning activities, and small scale testing attempts to answer this question: Is there reasonable probability that a successful product can be built? Yes. PLCs have been established in our district for the last 6 years. Our administrative team developed useful PLC tools, but they were never fully formalized and gathered into one place to ensure optimal use. We have experienced past successes, but a district handbook with all of the tools in one place would allow us to strengthen our implementation of PLCs. The activities, forms, graphic organizers, and data sheets in the handbook were designed into user-friendly (and print-friendly) versions and were added to the principals' professional development plan. The PLC handbook is now housed 
as a PDF on the Estacada School District's website to ensure easy access for principals and staff members (in case a hard copy version of the handbook is not readily accessible). The handbook is now a key document that will help drive the professional development for the district. The handbook will provide principals with learning activities, process descriptions, and protocols that can be used with staff. The PLC handbook activities were field-tested on a small scale in four schools at this step of the process.

3. Developing a preliminary form of the product helps to answer this question: Are personnel available who have the skills, knowledge, and experience necessary to build this product? The answer, again, is yes. The design team (four school principals and one vice principal) made up the primary personnel who were called upon because of their skills, knowledge, and experience with PLCs in schools. Three design team members had been principals at multiple levels (elementary and secondary), and were able to speak to the unique challenges of PLCs at each level. Design team members have also worked with a variety of staff members from high performing PLC schools. In addition, we have been in communication with Rick and Becky DuFour (the foremost national PLC experts) about the PLC work taking place in our district. We were also connected with other PLC leaders from around the state. For this step, we developed a preliminary form of the handbook, and looked for ways to refine the product. 
4. Preliminary field-testing attempts to answer this question: Can the product be developed within a reasonable period of time? The design team felt confident that it could establish a PLC handbook for our district. Many of the figures and templates for the handbook were already developed from past professional development activities. With additional input from the design team, we were able to develop and informally field-test the handbook. An initial draft of the handbook was reviewed with the design team members; it was then refined and formally field-tested it in the district's four schools. Design team members were able to use the handbook activities as a starting point for professional development with their staff, and adapt as needed to fit the unique needs and cultures of their schools.

5. Main product revision: After preliminary field-testing, the design team continued to meet on a weekly basis to gain a better understanding of what was working and what needed improvement. The handbook had sections that explained the PLC process. It also included activities that help promote positive group dynamics and cultivate teacher leadership. In addition, the handbook included activities to help schools address the current school change initiatives (DI/SI, RTI/PBIS, CCSS, and Proficiency-Based Learning). From design team sessions, we were able to understand how teachers and staff members in each building reacted to the use of the tools in the handbook. We discovered there were parts that were unclear to teachers and need revision. We address this important feedback during the main product revision step. 
6. Main field-testing: For the main field-test, the handbook was used in four schools. It was then refined based on feedback from the main field-test. At this point in the study, the design team transitioned from being a design team to a consultative focus group. For this step, the Critical Friends Consultancy Protocol (Harmony Education Center, 2013) was used during our weekly administrative team meetings. The Consultancy Protocol is a structured process that helps an individual or team think more expansively about a particular concrete dilemma or problem.

Table 4 outlines the protocol that was used to collect qualitative data for this study. The data collected from these meetings were transcribed, coded for themes, and analyzed. In each design team meeting where the Critical Friends Consultancy Protocol was used, I filled the role of Presenter. For the role of Facilitator, I asked team members to rotate through that role, which provide us with a new Facilitator for each meeting.

7. Operational product revision: The handbook was edited, refined, and made user-friendly in order to be operational for principals who intend to effectively guide the work of PLCs in their schools. For this step, we assessed and evaluated what we have learned as a result of the process we went through to develop the product, and our findings from preliminary and main field-testing. We reflected on our findings and presented recommendations for future practice 


\title{
Table 4
}

\section{The Critical Friends Consultancy Protocol}

\author{
Time: Approximately 50 minutes \\ Roles: Presenter (whose work is being discussed by the group) \\ Facilitator (who sometimes participates, depending on the size of the group) \\ Step 1: The presenter gives an overview of the dilemma with which he is struggling, and frames a \\ question for the Consultancy group to consider. The framing of this question, as well as the quality of the \\ presenter's reflection on the dilemma being discussed, are key features of this protocol. If the presenter \\ has brought student work, educator work, or other "artifacts," there is a pause here to silently examine \\ the work/documents. The focus of the group's conversation is on the dilemma. (5-10 minutes)
}

Step 2: The Consultancy group asks clarifying questions of the presenter-that is, questions that have brief, factual answers. (5 minutes)

Step 3: The group asks probing questions of the presenter. These questions should be worded so that they help the presenter clarify and expand his thinking about the dilemma presented to the Consultancy group. The goal here is for the presenter to learn more about the question he framed or to do some analysis of the dilemma presented. The presenter may respond to the group's questions, but there is no discussion by the Consultancy group of the presenter's responses. At the end of the 10 minutes, the facilitator asks the presenter to re-state his question for the group. (10 minutes)

Step 4:

The group talks with each other about the dilemma presented. (15 minutes)

Possible questions to frame the discussion:

What did we hear?

What didn't we hear that they think might be relevant?

What assumptions seem to be operating?

What questions does the dilemma raise for us?

What do we think about the dilemma?

Members of the group sometimes suggest actions the presenter might consider taking. Most often, however, they work to define the issues more thoroughly and objectively. The presenter doesn't speak during this discussion, but instead listens and takes notes.

Step 5: The presenter reflects on what was heard and on personal thoughts, sharing with the group anything that particularly resonated during any part of the Consultancy. (5 minutes)

Step 6: The facilitator leads a brief conversation about the group's observation of the Consultancy process. (5 minutes)

Source: adapted from the Harmony Education Center (2013) 
8. Operational field testing: This step was not pursued for the purposes of this dissertation. However, at this step in the process, the team would look to obtain additional feedback from a broader base of school communities and cultures. We ultimately want our handbook to be useful for principals in other schools, not just the schools in my district. With outside data and feedback, we will be able to revise the activities and tools that are unclear or difficult for teachers and staff from other schools. Since we intend to address the concerns of schools outside of our district, the refined handbook will be more useful for a greater number of schools.

9. Final product revision: This step was also not pursued for the purposes of this dissertation. However, at this step in the process, the design team would compile data and feedback from educational leaders from both inside and outside of our district, and apply what we learned to complete the final product revision.

10. Dissemination and implementation: This step was not pursued for the purposes of this dissertation. However, at this step, the design team would look to distribute the handbook to a wider audience so that they might also benefit from the findings of this study.

\section{Research Questions}

The study's primary research questions were: (a) Is the handbook for PLC leadership a useful resource for school leaders? (b) What are the handbook's strengths and weaknesses? The secondary research questions were focused on the specific sections 
and topics of the handbook: (a) How do principals organize and support a PLC framework? (b) How can PLCs support school change initiatives? (c) How can PLCs gather and analyze student data? (d) How can PLCs plan for future action? and (e) How can PLCs troubleshoot challenges?

\section{Data Collection Procedures}

In order to collect data, we used a design team approach. The design team was comprised of four principals from the Estacada School District and one vice principal. The design team assisted the main author of the handbook in the development, fieldtesting, and refinement of the product during the steps of the R\&D process. The design team met once a week, and discussed the handbook's usefulness and any field-testing challenges. The design team approach was advantageous because interaction among the participants yielded useful information and insight (Creswell, 2005). When facilitating the design process and field-testing stage, I encouraged all participants to talk and contribute their ideas, feedback, and constructive criticism regarding the product. Table 5 was adapted from the University of South Alabama's (2013) qualitative studies department. The table outlines the steps I used to code the data for this study. 


\title{
Table 5
}

\section{Coding and Developing Category Systems}

\begin{abstract}
Step 1: It is here that I carefully read my transcribed data, line by line, and divide the data into meaningful analytical units (i.e., segmenting the data). When I located meaningful segments, I coded them.
\end{abstract}

Step 2: Coding is defined as marking the segments of data with symbols, descriptive words, or category names. Again, whenever I found a meaningful segment of text in a transcript, I assigned a code or category name to signify that particular segment. I continued this process until I segmented all of the data and completed the initial coding. During coding, I kept a master list (i.e., a list of all the codes that were developed and used in the research study). Then, the codes were reapplied to new segments of data each time an appropriate segment was encountered.

Step 3: Corroborating and validating results is an essential component of data analysis and the qualitative research process. Corroborating and validating was done throughout the qualitative data collection, analysis, and write-up process. This was essential because I wanted to present trustworthy results to my readers. Otherwise, there was no reason to conduct a research study.

In addition to the primary data sources that came from creating, field-testing, and refining the handbook, 4 months of school PLC meeting minutes were available for review (all of the schools' data were available). This helped the design team get a sense of the challenges that were taking place in the PLC process at each school from the teachers' perspectives. It also highlighted the types of administrative support needed by PLC members throughout the course of a school year, as "administrative assistance requested" was one of the main headings of the PLC meeting minutes template used by the design team. The design team also engaged in conversations with teacher leaders on a weekly basis regarding their PLC activities. When available, teacher leaders were invited to participate in the Critical Friends Consultancy Protocol to provide data and feedback from a teacher's perspective. However, the key informants for this study remained the 
principals and vice principal who were using the handbook to guide the work of PLCs in their schools.

\section{Data Analysis Strategies}

The design team intended to make the PLC handbook meaningful for school leaders. We looked at organizational and structural changes. For example, we looked at how PLCs could be structured, through staff development activities provided by the principal, to address the major school change initiatives prevalent in today's educational landscape (DI/SI, RTI/PBIS, CCSS, and Proficiency-Based Learning). We determined which staff development activities included in the handbook were useful (including the accompanying graphic organizers, visuals, charts, and tables), and which should be modified or deleted all together. I coded my transcribed notes from our design team sessions, and also coded PLC meeting minutes in order to acquire data and feedback. Creswell (2005) defined the coding process as a qualitative research process in which the researcher makes sense out of text data, divides it into text or image segments, labels the segments, examines codes for overlap and redundancy, and collapses the codes into themes. The major sections of the PLC handbook, which data were coded for, included:

- The PLC Framework

- Supporting School Change Initiatives

- Content and Curriculum

- Collecting and Analyzing Data

- Planning for Future Action

- Troubleshooting Challenges 
Through this process, it was important for the design team to guard against bias and acknowledge our possible influence on the study's outcomes. We were extremely forthright and transparent in our approach. The five administrators who helped create, field-test, and refine the handbook knew full well that many of the resources and ideas came from our own workplace. Many of the big ideas for the handbook came directly from the Estacada School District, and the design team members had a hand their original conception. It was made clear by the design team that we were not trying to leverage our positions. What we wanted was an accurate assessment of whether or not the PLC handbook was useful. Design team members were asked to assess and evaluate whether or not the handbook was helpful for them in their work with PLCs in our district. However, design team members were also asked to think outside of our district, as we wanted our handbook to eventually be beneficial for principals in other schools too. Our school district is small and rural. We wanted the handbook to potentially benefit other small, rural schools. We also started to believe that the ideas and concepts in the handbook might also translate to other school settings (not just those in small, rural districts). To be sure, we hope to one day test the product in schools with demographics different from the ones found in our district.

\section{Work/Action Plan}

I requested and received a waiver from the Human Subjects Committee at Portland State University for this study, as there was no risk to the participants of the design team. This study did not look to review human subjects, it looked to assess and evaluate the effectiveness of a product (the PLC handbook). Preliminary research began 
in September 2011, as the design team began looking for a better way to formalize the resources and tools we were using as principals to guide the work of PLCs. We also began to formalize how we felt that PLCs could be used as the vehicle for facilitating all of the important mandated and highly recommended school change initiatives that we needed to implement (including DI/SI, RTI \& PBIS, CCSS, and Proficiency-Based Learning). Furthermore, we wanted to establish a systematic and comprehensive approach to PLCs in our schools that would cultivate teacher leadership.

In January 2013, four principals and one vice principal from the Estacada School District were invited to participate in this study. The study was discussed during an initial design team meeting, and their agreement to participate was secured. The design team members' feedback will remain anonymous. The members were informed that they were not required to participate in the study-their participation was completely voluntary. Participants were briefed on the nature of the study, and they received written information that included an outline of the data to be collected, as well as a description of the data collection activities and dates. Participants had the option to withdraw from the study at any time without any negative repercussions. The information from this study was coded to protect the identities of the participants. The coding ensured that the data collected had no impact on the participants' relationship with colleagues or supervisors. The design team explained its findings and made recommendations for future practice.

Figure 8 highlights the complete work plan timeline for steps $1-10$ of the R\&D process. 


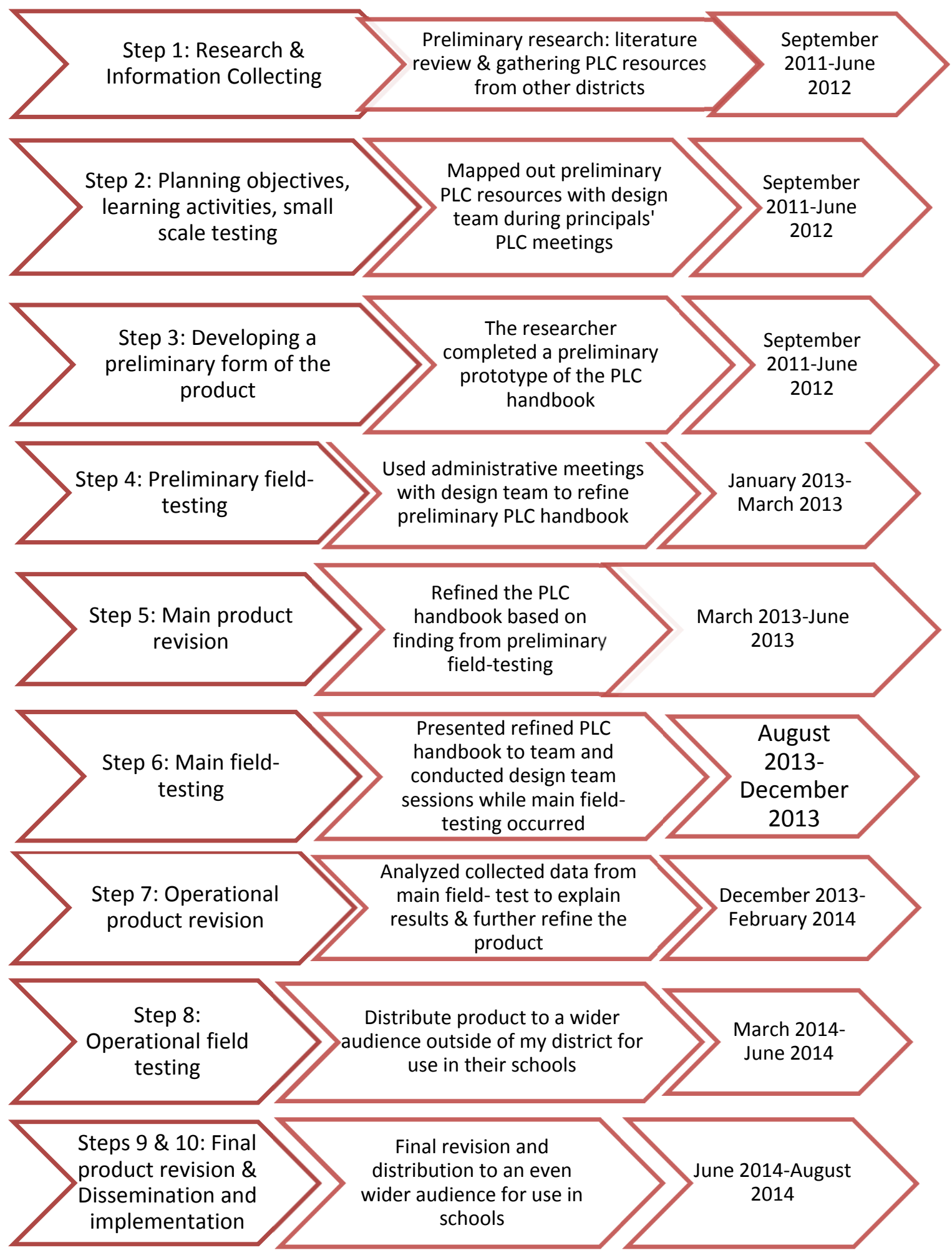

Figure 8. R\&D timeline. 
Much of the work for this study was already complete when I presented my dissertation proposal in May 2013. However, the completed work was only preliminary. Step six (the main field-testing of the handbook), which occurred from August 2013December 2013, was the most critical step. Defending my dissertation proposal by May 2013 was crucial so that, in consultation with the design team, I could spend the summer organizing and planning for the launch of the main field-testing when teachers returned to work in August 2013.

\section{Summary}

The PBL design (Bridges \& Hallinger, 1995) takes the findings generated by the basic and applied Research \& Development (Borg \& Gall, 1989) process and uses them to build and refine tested products that are ready for operational use in schools. As a design team, we created, field-tested, and refined a handbook for the leadership of PLCs in schools. The design team included four principals and one vice principal from the Estacada School District; the handbook was field-tested in four schools. The handbook provided the necessary materials for school leaders to facilitate and guide an effective PLC process in their school. The major sections of the handbook included: the PLC framework; supporting school change initiatives; content and curriculum; collecting and analyzing data; planning for future action; and troubleshooting challenges. Steps one through seven of the R\&D process were followed for the purposes of this dissertation. 


\section{CHAPTER 4}

\section{ANALYSIS}

\section{Introduction}

The purpose of this study was to create, field-test, evaluate and refine a handbook for PLC leadership. The study explored how school leaders can most effectively utilize and guide PLCs in their school and district. In the Estacada School District, PLCs are groups of educators who work together to address student needs and school change initiatives. Four principals and one vice principal served as a design team that created, implemented, and refined a handbook to guide the work of PLCs in four schoolsEstacada High School, Estacada Junior High School, Clackamas River Elementary, and Eagle Creek Elementary. Estacada High School is a comprehensive high school that serves 608 students. Estacada Junior High School serves 274 seventh and eighth grade students. Clackamas River Elementary has 590 students from kindergarten through sixth grade. Eagle Creek Elementary is also a kindergarten through sixth grade school and it currently serves 263 students. All schools are situated next to one another in a central campus except for Eagle Creek Elementary, which is approximately six miles away. For additional background knowledge, it should be noted that there is a vacant elementary school (formerly River Mill Elementary) located at the central campus, which could be used by the district to serve students in the future.

In Estacada, school principals guide the work of PLCs; the problem is that implementing PLCs effectively is a challenge. To address this problem, the design team 
developed, field-tested, evaluated, and worked to improve a PLC handbook. The handbook activities were intended to strengthen school and district PLC work. During the main field-testing of the handbook, dedicated time for PLC work was allotted to staff the last 2 hours of every Friday. Students across the district were released 2 hours early every Friday to allow for this staff PLC collaboration time. During the dedicated PLC time, staff participated in, and completed, activities from the handbook that was developed by the design team.

This chapter describes the findings from the data collected during the study. Most of the data were collected through qualitative feedback from design team sessions that were dedicated to evaluating and improving the effectiveness of the handbook. The Critical Friends Consultancy Protocol (Harmony Education Center, 2013) was used during design team sessions, which allowed the team to collect and analyze data. The Critical Friends Protocol is a six-step process that was used to obtain qualitative data and feedback from design team members. The protocol used by the design team is described in brief below:

- Step 1 (5-10 minutes): The presenter (the main author of the handbook) gave an overview of the problem (that implementing PLCs effectively is a challenge), and framed a question for the consultancy group to consider. For this study, the guiding question for each session was: Is the handbook, in its current state, helpful to you and your staff in guiding the work of PLCs, and how could it be improved? The design team also reviewed student work, educator work, and other artifacts relative to PLCs during step one. 
- Step 2 (5 minutes): The design team asked clarifying questions of the presenter.

- Step 3 (10 minutes): The design team asked probing questions of the presenter. The presenter responded to the questions, but there was no discussion by the group at this point. At the end of the 10 minutes, the presenter restated his question for the group.

- Step 4 (15 minutes): The design team talked with each other about the problem presented. The presenter did not speak during this 15 minute period of time, but instead listened intently and took notes.

- Step 5 (5 minutes): The presenter reflected on what he heard, and shared with the group anything that particularly resonated for him during any part of the protocol.

- Step 6 (5 minutes): The facilitator (this role was rotated around so that each design team member had a chance to facilitate) led a brief conversation about the group's observation of the protocol.

The main field-test of the handbook occurred from August 2013 to December 2013. The most significant and informative findings were discovered as the handbook was fieldtested on Fridays district-wide during this period of time.

Descriptions of adaptations made to the handbook as the R\&D process unfolded will also be included in this chapter. In addition to design team sessions, data were gathered by design team members as they reviewed weekly PLC meeting minutes submitted by their staffs. The review of these documents allowed design team members 
to gain a better understanding of the handbook's effectiveness, and informed the discussion that took place during design team sessions. Design team members also attended, and participated in, several PLC meetings throughout the course of the field test; which informed their perspectives during their sessions together. In addition, design team members reflected on their experiences leading staff development using the handbook's activities. The design team identified common trends and themes in their data collection to analyze and evaluate the handbook's effectiveness. Quantitative data, which are described in more detail later in this chapter, were also gathered and analyzed through the use of surveys. The design team used their data and findings to improve the Estacada PLC handbook.

\section{Review of Research Goals/Questions and General Design of the Project}

\section{The R\&D Process}

To develop, evaluate, and improve the Estacada PLC handbook, the design team conducted the first seven steps of the problem-based process outlined below.

- Step 1-Research and information collecting

- Step 2-Planning objectives, learning activities, and small scale testing

- Step 3-Develop a preliminary form of the product

- Step 4-Preliminary field-testing

- Step 5-Main product revision

- Step 6-Main field-testing

- Step 7-Operational product revision

- Step 8-Operational field-testing 
- Step 9-Final product revision

- Step 10-Dissemination and implementation

The study relied on the work of Bridges and Hallinger (1995) who recommended the use of an R\&D cycle developed initially by Borg and Gall (1989, pp. 784-785). It consists of 10 steps, the first seven of which are relevant to this study. At each step, the design team conducted research that led to the next stage of the product or handbook development. The R\&D process was enacted through qualitative research involving data provided in design team sessions, as well as quantitative data gathered through the use of surveys. The design team met weekly, as proposed in chapter three. However, due to increased workloads, time did not permit use of the full Critical Friends Consultancy Protocol every single week. To address this challenge, the design team agreed to hold six uninterrupted sessions, exclusively dedicated to the evaluation of the PLC handbook's effectiveness, using the Critical Friends Consultancy Protocol, as the handbook was being field-tested.

The protocol was a structured process that helped design team members evaluate the handbook and think more expansively about the challenging problem of implementing PLCs. The design team sessions that utilized the protocol resulted in rich discussion about handbook improvement, and the team felt that six full sessions were sufficient to bring the product through step seven of the R\&D cycle. Design team sessions were informed by data collected through the review of survey data, the review of PLC work submitted by school staffs, observations by principals during school PLC meetings, and reflection of staff development activities guided by the handbook. The design team sessions that used the protocol were tremendously informative and provided 
valuable insights. In addition to the six design team sessions that used the protocol, the team met weekly (and minutes were kept). Even though the protocol was not used in every single team meeting (due to time restrictions), these meetings often featured discussion of PLC-related items. Therefore, the weekly team discussions and minutes were extremely helpful in identifying common themes and elements to help inform handbook refinement and improvement.

\section{Development and Implementation (Field-Testing) of the PBL Project: Description of the Design Team's Experience Through Step Seven of the R\&D Process}

This study followed the commonly used R\&D methodology to develop and evaluate the Estacada PLC handbook. I conducted a literature review that informed the design team's initial drafts of the PLC activities to be included in the handbook. I also transcribed notes from design team sessions that utilized the Critical Friends Consultancy Protocol. Design team members reviewed local documentation, including weekly PLC minutes and copies of work produced by staff who participated in PLC handbook activities. After transcribing the notes from the design team's meetings, I coded the notes to discover themes and elements within the data. By finding patterns, and making connections within the data, the design team was able to answer difficult questions and make sense of the data to refine the handbook. A description of the qualitative data analysis process that was used follows.

Creswell (2005) described coding as a process of segmenting and labeling text to form descriptions and broad themes in the data. Through coding, common ideas are discovered and connections are made among ideas so as to allow the ideas to merge 
together into themes. The themes that emerged from the data were derived from the development and refinement of the handbook.

\section{General Design of the Handbook}

The handbook was explicitly designed to fit the circumstances of the design team's work and meet the needs of the district. We made adaptations to many aspects of the handbook in order to create a better product. We aimed to produce a handbook that moved the district forward in its use of PLCs. While preliminary research showed that building PLC capacity for educators in the district was likely to be useful, we were surprised by the level of interest shown by non-educator stakeholders (i.e., families and community members) during the field-test. We ended up eventually expanding the handbook to include ways for families and community members to become involved in PLC work, but that occurred toward the end of the R\&D process, and still requires further R\&D.

The aim of the design team was to provide educators with common language and practical tools to use during their work in PLCs. The handbook that was used for the main field-testing included 15 activities that addressed the key areas outlined below.

- The PLC framework

- Content and curriculum

- Collecting and analyzing student data

- Supporting school change initiatives

- Planning future action

- Troubleshooting challenges 
After the operational product revision occurred (step seven of the R\&D cycle), the handbook grew to include 22 activities.

\section{Steps of the R\&D Process and Incremental Data Analysis}

The R\&D process allowed the design team to discover themes and elements in the data at each step of the process. The data collected led to discussion of ongoing fieldtesting challenges. The following analysis of each R\&D step will highlight findings and conclusions. The analysis of the data collected at each step will characterize what the design team learned. The issues and challenges encountered will be described in the context of the specific step in the process. The content and activities in the handbook were conceived of as a means of providing support and specific materials needed for PLCs to complete their work. The design team completed the initial draft of the handbook, aiming to address the most common concerns associated with PLCs, such as explaining the tasks that PLCs should be completing as they work together.

Early on in the R\&D process, the design team recognized that data would need to be collected from the handbook's primary end-users; the teachers. We needed to better understand teachers' unique approaches, and know the questions they were asking about PLCs and the handbook. We needed the teachers' collaboration and advice. The R\&D process was the appropriate methodology to work through our challenges and problems. Through field-testing, the design team gained a better understanding of PLCs' needs, questions, and concerns, which helped us uncover the best ways to improve the handbook. Working through the R\&D process, particularly completing the main fieldtest, enabled the design team to develop a more usable and effective product. 


\section{Step One: Research and Information Collecting}

In the spring of 2012, to drive professional development activities in the Estacada School District, the administrators (including principals and the superintendent) determined that a handbook was needed to guide the work of PLCs. Consequently, a design team was formed to develop a PLC handbook for the district. The initial step of research and information collecting defined the problem that PLCs are difficult to implement. This initial step also highlighted that a PLC handbook, as an educational tool, could help address the problem. The methods used by the design team at this step of the R\&D cycle included a review of the published literature on PLCs, a review of past and current PLC work, and field observations of PLC meetings at the four schools. Through research and information collecting, the design team felt confident in making the claim that PLCs are a widely-accepted and recommended practice for schools. The problem is that PLCs are difficult to implement, and this problem provided the impetus for the design team's handbook.

The product emerged from a review of the literature, a review of PLC work, and field observations, but also from the design team paying attention to its surroundings and cultural norms at work. Researching and collecting information regarding the problem and educational need that the product was meant to address greatly informed our understanding of important aspects of PLC work. As school leaders, we wanted to know how we could organize and support PLCs by creating a handbook that clarified PLC language and activities for use across the district's schools. In the handbook, we also wanted to demonstrate that PLCs could support current school change initiatives. The 
design team understood that the handbook would need to move our district forward in its use of PLCs.

As school and district leaders, we had access to many current and historical documents related to PLCs. We also had access to PLC tools and resources from other districts. In addition, each design team member attended a wide variety of PLC trainings and leadership institutes. Furthermore, on a regular basis, design team members participated in many discussions with key school and district personnel relative to PLCs.

At step one of the R\&D cycle, it was important for the design team to understand the current reality of the Estacada School District, including its significant challenges. Estacada currently faces a variety of issues that impact PLC work, the most notable being its declining enrollment. On average, each student brings approximately $\$ 6,000$ to the district. Fewer students translate into fewer dollars for the district to spend on staff and resources. The challenge of declining enrollment has complicated PLC work.

There are currently imbalanced student numbers, staff, and resources-particularly at the district's two elementary schools, which both serve students K-6. Clackamas River Elementary currently has 590 students, and is organized into single grade classrooms. Eagle Creek Elementary, on the other hand, has 263 students, and is organized into "split" classrooms (i.e., first/second, third/fourth, fifth/sixth). Kindergarten is the only single grade classroom currently at Eagle Creek Elementary. Due to the current configuration of Estacada's elementary schools, each school has its own unique challenges when working in PLCs. For example, Eagle Creek Elementary’s main challenge was that most teachers working in PLCs were not able to focus on teaching a 
single grade level. When Eagle Creek Elementary's PLCs met to collaborate, they had to account for two sets of CCSS, rather than focusing on one.

Despite the various and unique challenges that existed at each school, the design team was determined to develop a handbook that could be used district-wide. Each school principal adapted the activities in the handbook to fit the specific needs and culture of their school. These ideas formed the foundation from which the design team researched PLCs as a topic for study. The design team's intent was to conceptualize a PLC handbook that would be useful to the schools and district.

At step one of the R\&D cycle, the ideas for the handbook included research relative to how instructional technology could assist PLCs. The design team gathered input and additional data from Estacada's two charter schools-Estacada Web Academy and Estacada Early College. Both of these district-sponsored charter schools operate primarily in a virtual environment. More than 850 students currently attend Estacada's charter schools, and only $9 \%$ of those students live within the boundaries of the Estacada School District. The design team's collaboration with its charter schools greatly informed its work in developing the handbook. We learned more about instructional technology by collaborating with our charter schools, and will continue to explore ways to maximize the benefits of this relationship. Informants from the charter schools included three administrators (one director, one principal, and one associate principal), as well as a variety of teachers and support staff. The district's charter schools specialize in online education, so they were well-versed in PLC collaboration that included instructional technology. As the ideas for the handbook continued to evolve, the design team gathered 
a considerable amount of feedback, which was included in subsequent handbook revisions and small-scale testing.

The most frequently identified element that influenced the design team's perspective on the development of the handbook was our district's readiness for change, in terms of how a new district-wide PLC handbook would be received. Through our administrative training, we have developed extensive knowledge of the principles and practices of learning, organizational leadership theory, and the policies and politics of education-all of which are relevant to PLC work. In addition, over the past several years, design team members have become familiar with school change initiatives that have surfaced, many of which are either mandated or highly recommended by well-regarded leaders in the field of education. National and state policy makers are calling for increased accountability. In Estacada, this call for increased accountability has forced the design team to reexamine how our schools operate as PLCs.

The educational need for a PLC handbook was solidified in the minds of design team members during the research and information-collecting step of the R\&D process. The team agreed that teachers and support staff believed PLCs could improve at each school throughout the district. The design team was also convinced that a more consistent approach to PLCs district-wide was desirable, and that a handbook could provide direction. Teachers and staff confirmed the design team's perspective that the unique culture of each school should not be ignored. While each school in the district could strive for a similar approach to PLCs, the handbook activities would need to be adaptable to fit each school. The design team also agreed that most staff appreciated working in 
PLCs, as it has provided them with time to address student needs and complete the work required of current school change initiatives.

The four main school change initiatives currently addressed in Estacada are described in detail in chapter two. These school change initiatives, which are either mandated or highly recommended, include:

\section{DI/SI}

\section{RTI/PBIS}

\section{CCSS}

\section{Proficiency-Based Learning}

From their conversations with staff, design team members revealed during their sessions together that the majority of educators in the district did not view these initiatives as optional, rather a required part of their work. All five design team members noted that most staff members used the majority of their PLC time completing work that helps address student needs and keep our schools and district in compliance with the state and federal guidelines. Design team members also indicated that most staff felt working in PLCs gave them much-needed time to address the current school change initiatives, but that it was still a challenge to complete all of the required work. The design team needed to develop a handbook that allowed staff to be more productive and efficient in using their PLC time to address current school change initiatives. The design team members' were well trained and capable of producing high quality instructional materials. Developing a handbook would enrich what we were already doing in our schools to guide PLC work. 
In addition to becoming more productive and efficient, commentary from design team sessions pointed to perceptions about motivation and getting involved in the PLC process. One design team member stated, "Members must be intrinsically motivated to be involved in PLCs or else it will not happen." Another design team member commented, "Teachers appeared to be satisfied after PLC work occurred among staff from multiple schools as they collaborated to align curriculum." A significant finding at this step was that small school districts have the potential to maximize the benefits of PLCs; perhaps more so than large school districts because of their added layers of bureaucracy that can lead to complex challenges which are difficult to navigate. Most design team members had years of experience in larger school districts, and thus were able to confidently make these comparisons and draw conclusions. By maximizing the potential benefits of PLCs in Estacada, the district could become stronger and more effective. The idea of bringing staff members together from multiple schools in our small district, to engage in PLC work, fostered debate among educators and led to the expression of assumptions and beliefs. The uncovering of these assumptions and beliefs allowed the design team to explore and analyze differing viewpoints about the district's use of PLCs.

Through design team discussions, a theme that emerged was that there was high interest by educators in the PLC process, but low capacity due to increased workloads. This finding affirmed the design team's assumption that a PLC handbook could serve as a useful solution to the challenge of increased workloads. When asked directly what would help support them in PLCs, school staff reported wanting help with role identification, as well as training that addressed important initiatives. Training on role definition and 
important school change initiatives, through use of the handbook, had the residual effect of building both school-wide and district-wide culture. Another finding was that the widespread use of the PLC handbook, through field-testing, engaged many educators in discussions and actions relative to PLC improvement.

A comprehensive PLC handbook could drive professional development in Estacada. The design team aimed to develop a handbook that was useful and would allow an academic process of inquiry to emerge that connected educators to the spirit of collaborative PLC work. The data collected through transcribed notes from weekly design meetings demonstrated that a handbook to guide the work of PLCs was an educational product that had promise. One design team member noted that Estacada needed to get "back to basics" with how PLCs operated. Having useful tools and activities available in a PLC handbook appeared to have value.

\section{Step Two: Planning Objectives, Learning Activities, and Small-Scale Testing}

The second step of the R\&D process involved planning objectives, learning activities, and small-scale testing. The design team identified specific learning objectives for staff that could be achieved through use of the handbook. For example, the design team knew it wanted the handbook to spell out the various tasks that PLCs would work to complete. The PLC tasks would have to be outlined in a clear and understandable way so that people new to the PLC process would have direction; this would also provide review for veteran PLC members. The design team also worked to define the skills necessary for PLC work, and intended to highlight those skills in the handbook. It was important for the handbook to include professional development activities that had received positive 
feedback from PLCs when used in small-scale testing. The design team wanted to formalize and improve PLC-related activities that had already led to desirable outcomes for PLCs.

An emergent product was developed by collecting PLC activities that design team members had used in the past with their staffs, such as activities to help PLCs identify essential learning targets and develop common assessments. Estacada had been operating as PLCs for 6 years leading up to the main field-test of the handbook. Hence, design team members had used a number of PLC activities in the past to guide professional development, but they had never been compiled and housed in one place for optimal use. Design team members used and refined the PLC activities they already had, which served as small-scale testing for the team. Through small-scale testing, the team proposed to better understand the instructional needs of staff related specifically to their collaborative work in PLCs. We also hoped that if families and community members read the handbook we created, they would have a better understanding of key PLC terminology and activities. We needed to ask critical questions to understand how district and community stakeholders viewed the work of educators in our district. It was important for all educators in our district to know what the Estacada residents valued, needed, and wished to know about the PLC process and its implications for the district's future.

Among many data sources, design team members used their experience and observations as educational administrators, and professional development leaders, to form their assumptions about what the district needed to move forward with PLCs. We observed each other's past and present approaches to professional development. We 
listened to each other's critiques of attempts at school reform, and worked to provide for professional development needs within the instructional design of the handbook. A common theme identified from the design team's weekly meetings was that there was interest in PLCs from a cross-section of educators throughout the district. Most educators expressed a desire to improve PLCs, but were not sure where to start or how to move the process forward. The design team needed to translate educators' concerns about how to start and how to move forward into a handbook that would provide clarification and direction. The data collected and analyzed at step two affirmed some of the design team's assumptions and contradicted others.

The most frequently identified elements and variables that influenced the handbook's development at step two included PLCs' needs from administration in terms of support. As the design team planned objectives, developed handbook activities, and conducted small-scale testing, the team opted to survey staff. As the survey data were collected and reviewed, design team members brought what they learned to the design team sessions. One of many small-scale testing examples included use of the "PLC Survey: What is Your Current Reality?" The PLC survey served two purposes for the design team:

1. To collect data and gain a better understanding of PLCs' needs. The data collected from this survey were analyzed by the design team, and informed future revisions of the PLC handbook. 
2. To collect data and feedback regarding the survey itself. This survey would be included in the PLC handbook, so small-scale testing helped the design team improve this activity for inclusion in future editions of the handbook.

The design team wanted to see if its survey of PLCs was useful in gathering information about the assumptions, concerns, and needs held by the staff. The team also wanted to ensure that the survey could be given at various checkpoints in the year to gauge the progress of PLCs. The data acquired from this survey led to rich discussion during design team sessions relative to PLCs' current reality, as well as their needs for the future. The survey that was used in small-scale testing is included in Table 6, and includes the average score of the respondents.

Based on understanding gained through the design team's analysis of the survey results and comments, the team uncovered important aspects related to staff needs, specifically how administration could provide assistance and direction for PLCs. The design team concluded that most educators perceived professional development through the PLC process as important. Therefore, fidelity to the PLC process was valued, and a handbook could help guide future work. The findings from the design team's data analysis of the survey results and comments are summarized below. The data revealed that educators readily accepted the need to:

- Identify essential learning targets

- Improve instruction

- Use assessment data to analyze student results

- Provide intervention for struggling students

- Provide enrichment for advanced students 


\section{Table 6}

\section{PLC Survey: What is Your Current Reality?}

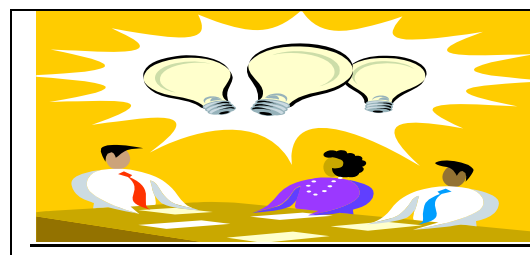

\section{PLC Survey: What is our Current Reality?}

Mark $\mathbf{1}$ for "Not True of Our Team"

Mark 2 for "Our Team is Addressing This"

Mark 3 for "True of Our Team"

1. $3 \mathrm{We}$ have identified team norms and protocols to guide us in working together.

2. 2 We have established SMART goals to help us understand what we would like to achieve with our students (SMART goals = Strategic, Measurable, Attainable, Results-Oriented, and Time-bound).

3. 2 We have aligned our priority standards (from the CCSS) with the test specifications of our state assessment.

4. $2 \mathrm{We}$ have agreed on how to best sequence the content of our course(s) and have established pacing guides to help students master the intended priority standards.

5. 2 We have identified the prerequisite knowledge and skills students need in order to master the priority standards.

6. 2 We have identified strategies and created instruments to assess whether students have the prerequisite knowledge and skills.

7. $2 \mathrm{We}$ have developed interventions to assist students in acquiring prerequisite knowledge and skills when they lack in those areas.

8. 2 We have developed frequent common formative assessments that help us to determine each student's mastery of priority standards.

9. 2 We have established the level of proficiency we want to see from students for the priority standards.

10. 2 We use the results of our common assessments to assist each other in building on strengths and addressing weaknesses as part of an ongoing process of continuous improvement designed to help students achieve at higher levels.

11. 2 We use the results of our common assessments to plan intervention and enrichment activities for students.

12. 2 We have taught students the criteria we will use in judging the quality of their work and provided them with examples.

13. 3 We have developed or utilized common summative assessments that help us assess the strengths and weaknesses of our program(s).

14. 2 We use our PLC time to address DI/SI, PBIS/RTI, CCSS, and Proficiency-Based Learning.

15. 2 We formally evaluate our adherence to our team norms and the effectiveness of our team at least twice a year. 
Another finding was that educators acknowledged the need to focus on the four current school change initiatives (DI/SI, RTI/PBIS, CCSS, and Proficiency-Based Learning). Respondents to the survey indicated that teachers believed fidelity to the PLC process would lead to school improvement. Throughout the design team's discussions, a common theme noted was that staff members appreciated feeling supported in their PLC efforts by the top leadership in their school and district. Consequently, the design team aimed for the PLC handbook to be supportive of faculty-led PLC efforts.

The design team determined that a PLC handbook would address gaps in knowledge and skills, and we began to develop our handbook into a more formalized product. Design team members characterized conversations they had with the members of their staff, as well as comments provided from the survey, regarding PLC priorities. One design team member stated, "Staff felt that addressing Differentiated Instruction/Sheltered Instruction through the revision of a walkthrough tool would fill a gap.” Another member noted, "The continued melding of Response to Intervention and Positive Behavioral Intervention Support into what many districts are now calling EBISS would fill a gap and streamline the work required of teachers and staff." Another member stated, "PLC activities focusing on the rollout of the new CCSS would be beneficial, particularly with students required to take new state tests (SMARTER Balanced Assessments) based on the CCSS in 2014-15." Yet another design team member stated, "PLC activities should address Proficiency-Based Learning, particularly with the recent adoption of Oregon House Bill 2220, which mandates schools and districts to implement Proficiency-Based Learning by the end of 2013-14." Addressing these suggestions 
became the design team's main objectives as we developed the handbook into an emergent product. The design team used its data analysis and findings from step two to develop a preliminary form of the PLC handbook.

\section{Step Three: Developing a Preliminary Form of the Product}

Step three of the R\&D cycle included the preparation of instructional materials and evaluation methods for the first presentation of the product (Lorenz \& Pichert, 1989). Design team members determined the appropriate scope, sequence, style, and tone of the handbook. The team also discussed the evaluation criteria to be used in preliminary fieldtesting. At step three, the design team reviewed PLC handbooks and activities from other school districts, including Parkrose, Oregon City, Oregon Trail, Molalla, Lincoln County, Centennial, David Douglas, Gresham-Barlow, Tigard-Tualatin, Corvallis, and Rainier.

The team also reviewed PLC activities produced by the educational organization Solution Tree. Solution Tree is well known for its PLC Leadership Institutes. All design team members had received training through at least one Solution Tree PLC Leadership Institute; many had participated in multiple institutes.

Since all design team members had experience with PLC leadership, the team was able to complete a comprehensive literature review of PLCs, and discuss their findings in design team sessions. Through this data analysis, the design team gained an understanding of what a quality PLC handbook should include. Several themes and elements were uncovered relative to the scope, sequence, and formatting that proven handbooks included. One of the main findings was that the quality of PLC handbooks from other districts varied considerably. Very few PLC handbooks provided 
comprehensive information about the process in terms of how it may unfold-particularly in a small school district. Many handbooks discussed aspects of the PLC process, but were general in their discussion. In some cases, the PLC handbooks reviewed were designed to speak to an audience of educators working in districts much larger than Estacada. Nevertheless, the research was clear that fundamental ideas for PLCs exist, and should be applied. However, a challenge for the design team was to put theory into practice in Estacada in a realistic and practical way. The Estacada PLC handbook needed to be educationally sound and comprehensive, and also consider Estacada's unique needs.

The design team confirmed its assumption that the PLC handbook needed to give educators an understanding of current school change initiatives. Many of the PLC handbooks and activities from other districts included information on DI/SI, RTI/PBIS, CCSS, and Proficiency-Based Learning. However, the design team found some of the handbooks and activities to be dry and impractical. Many seemed to be a collection of administrative tasks that teachers would presumably complete and turn in to their principal. The design team found this tone to be off-putting and uninspiring. The activities in the Estacada PLC handbook needed to be user-friendly. The handbook had to assist users in the work they were already doing and help them become more efficient and productive.

In the $50^{\text {th }}$ anniversary issue of the Ohio State University's refereed journal, Theory Into Practice, Gaskill and Hoy (2013) reminded us that educational theory has shown an interesting progression through the recent history of school change initiatives. One goal for Theory Into Practice has been to keep their writings free of educational 
jargon and technical terms to the greatest extent possible, making content accessible to practitioners and others not deeply grounded in the field of education. The Estacada design team followed Theory Into Practice's advice at step three as it developed the preliminary form of its PLC handbook in a manner that would be user-friendly.

From their discussions, the design team agreed that educational theory related to PLCs would need to be included in the handbook, but that it should be at a basic and easily accessible level. Another finding was that the handbook should include ways for users to dig deeper into educational theories associated with PLCs. Educators who wished to dig deeper became prime candidates to serve as teacher leaders of PLCs during the preliminary and main field-testing. Because the design team wanted to encourage and increase teacher leadership, a collegial tone was used in the preliminary form of the handbook. I. Clark (2011) affirmed that people attend to conversational tones better than procedural or authoritarian tones. The collegial tone of the preliminary form of the handbook was established to promote rich discussion that would ultimately lead to action.

Another part of step three was to establish evaluation procedures for the preliminary field-testing of the handbook, as well as the design team's group process. In researching and developing the handbook, the design team discovered a useful resource that spoke to both the content and evaluation of handbooks, as well as group process. Evanson's (2013) rubric from her work with the Eberly Center for Teaching Excellence and Educational Innovation at Carnegie Mellon University was reviewed, adapted, and used to evaluate the handbook and our design team's process as measured by three levels of achievement-sophisticated, competent, and not yet competent. The advice garnered 
from the Evanson rubric led to a significant finding; that field-testing the handbook with intended users was important, as it provides significant insights on usability, applicability, and accessibility. The aim of the design team was to reach a "sophisticated" level of achievement for each key area of the rubric by the time it completed step seven of the R\&D cycle. The adapted version of the Evanson rubric used by the design team is included in Table 7 with average numerical scores; this rubric was used for subsequent preliminary field-testing and evaluation of the Estacada PLC handbook, and the design team's group process.

\section{Step Four: Preliminary Field-Testing}

The purpose of the preliminary field-testing was to obtain an evaluation of the initial product (Gall et al., 1996). Step four of the R\&D cycle emphasized a qualitative assessment of the handbook's content and format. Direct observations were made by principals of their staff members during PLC meetings that utilized activities from the handbook. Data and feedback were gathered by design team members at each school through the review of weekly PLC minutes submitted by school staff. The direct observations (and subsequent notes) from principals, as well as the feedback from PLC members at each school, served as the primary data sources for design team meetings regarding issues such as clarity of instructions and materials, adequacy of time allotted for activities, and maintenance of participant interest. Design team members presented their staffs with a preliminary form of the product. Since the handbook would impact school and district culture, the design team wanted to verify the readiness of its schools, as well as the content, scope, sequence, tone, and style of the material within the 
handbook. The design team needed to verify that the PLC activities would indeed be

effective in meeting the outcomes the schools and district intended.

Table 7

Evaluation Rubric for Development of the Estacada PLC Handbook

\begin{tabular}{|c|c|c|c|}
\hline \multicolumn{4}{|c|}{ Levels of Development } \\
\hline Criteria & Sophisticated (3) & Competent (2) & Not Yet Competent (1) \\
\hline $\begin{array}{l}\text { Clarity of the } \\
\text { Handbook's } \\
\text { Direction (3) }\end{array}$ & $\begin{array}{l}\text { The purpose of the } \\
\text { handbook is clear and is } \\
\text { tied to research }\end{array}$ & $\begin{array}{l}\text { The purpose of the } \\
\text { handbook is clear, but } \\
\text { only partially tied to } \\
\text { research }\end{array}$ & $\begin{array}{l}\text { The purpose of the } \\
\text { handbook is confusing } \\
\text { and is not tied to research }\end{array}$ \\
\hline $\begin{array}{l}\text { Quality of the } \\
\text { Handbook (3) }\end{array}$ & $\begin{array}{l}\text { Good data collection-- } \\
\text { information and content is } \\
\text { accurate; sources are } \\
\text { legitimate; activities are } \\
\text { appropriate }\end{array}$ & $\begin{array}{l}\text { Information and content } \\
\text { is mostly accurate; } \\
\text { sources are good but } \\
\text { not varied enough; } \\
\text { some activities may be } \\
\text { questionable }\end{array}$ & $\begin{array}{l}\text { Information and content is } \\
\text { unreliable and/or } \\
\text { inaccurate; sources and } \\
\text { activities are not valid }\end{array}$ \\
\hline $\begin{array}{l}\text { The Handbook } \\
\text { Effectively } \\
\text { Communicates } \\
\text { Content Directions } \\
\text { (3) }\end{array}$ & $\begin{array}{l}\text { The activities included in } \\
\text { the handbook bring } \\
\text { opportunities to life for } \\
\text { staff }\end{array}$ & $\begin{array}{l}\text { The activities are } \\
\text { uneven and take a lot of } \\
\text { explanation to } \\
\text { communicate; it is } \\
\text { difficult to imagine } \\
\text { actual use }\end{array}$ & $\begin{array}{l}\text { The activities included in } \\
\text { the handbook do not get } \\
\text { the ideas or intent across } \\
\text { to the audience }\end{array}$ \\
\hline $\begin{array}{l}\text { Product Evaluation } \\
\text { (3) }\end{array}$ & $\begin{array}{l}\text { Team used systematic } \\
\text { testing to validate and } \\
\text { drive refinement }\end{array}$ & $\begin{array}{l}\text { The team used very } \\
\text { informal feedback to } \\
\text { drive refinement }\end{array}$ & $\begin{array}{l}\text { No test or feedback was } \\
\text { used }\end{array}$ \\
\hline $\begin{array}{l}\text { The Team's } \\
\text { Reflection of the } \\
\text { Handbook's Design } \\
\text { (3) }\end{array}$ & $\begin{array}{l}\text { The handbook explains } \\
\text { the activities well, } \\
\text { provides clear } \\
\text { instructions, does not use } \\
\text { jargon, and highlights key } \\
\text { elements }\end{array}$ & $\begin{array}{l}\text { The handbook is } \\
\text { coherent for the most } \\
\text { part, but missing one or } \\
\text { two key elements }\end{array}$ & $\begin{array}{l}\text { The handbook lacks } \\
\text { coherence and is missing } \\
\text { three or more important } \\
\text { elements }\end{array}$ \\
\hline $\begin{array}{l}\text { Analysis of Design } \\
\text { Team Process and } \\
\text { Individual Roles } \\
\text { within it (3) }\end{array}$ & $\begin{array}{l}\text { Team members clearly } \\
\text { articulated: 1) What } \\
\text { worked well and why; 2) } \\
\text { What did not work well } \\
\text { and why; and 3) Ways to } \\
\text { increase effectiveness and } \\
\text { efficiency of group } \\
\text { process in the future, } \\
\text { considering self as well as } \\
\text { others }\end{array}$ & $\begin{array}{l}\text { Team members } \\
\text { discussed only two of } \\
\text { the three; discusses } \\
\text { group without } \\
\text { discussing self; } \\
\text { discusses self without } \\
\text { discussing group }\end{array}$ & $\begin{array}{l}\text { Team does not articulate } \\
\text { any of the three-what } \\
\text { worked well and why; } \\
\text { what did not work well } \\
\text { and why; how to improve } \\
\text { the process }\end{array}$ \\
\hline $\begin{array}{l}\text { Participation by } \\
\text { Design Team } \\
\text { Members during the } \\
\text { R\&D Process ( } 2)\end{array}$ & $\begin{array}{l}\text { Active participation in the } \\
R \& D \text { of the handbook }\end{array}$ & $\begin{array}{l}\text { Some participation in } \\
\text { the R\&D of the } \\
\text { handbook }\end{array}$ & $\begin{array}{l}\text { Little participation in the } \\
\text { R\&D of the handbook }\end{array}$ \\
\hline
\end{tabular}


Preliminary field-testing began in the winter of 2013. While a preliminary form of the product was presented to all staff members, the design team felt it needed to determine a subgroup of educators who would have valuable input about the appropriateness of the handbook, and thus should be more heavily involved in the preliminary field-testing. We needed to find the right subgroup members who had expertise and interest in particular components of the handbook to ensure that the intent and messages would be embraced by PLCs. We used our past experience and knowledge of the district to determine the appropriate population to approach for more in-depth participation in the preliminary field-testing. It was important that the handbook took an appropriate approach that would lead to school and district reform. To this end, the design team identified key informants: well-regarded teacher leaders in each school who had demonstrated high levels of performance in past PLC activities.

The design team continued to facilitate deep review and discussion regarding the effectiveness of the PLC handbook through its weekly design team meetings. This feedback gave us a good sense of how the PLC handbook activities would move forward in each school when main field-tested. Design team members communicated with their key informants on a regular basis, both in person and via e-mail. In order to protect the rights of the human subjects who participated in the preliminary field-testing, individual participants and their comments are not identifiable as recorded in this study. Design team members acquired relevant information and data and shared it with the team. The design team sessions that used the Critical Friends Consultancy Protocol (described earlier in this chapter) proved to be most valuable during preliminary field-testing 
because it provided an environment free of distractions; this allowed us to focus more intently on the effectiveness of the PLC handbook. In these sessions, we also used our rubric (adapted from Evanson's work) to rate the effectiveness of both our handbook and our team's process as we moved through the R\&D process. Our goal, again, was to reach a "sophisticated" level of achievement in all key areas of the rubric.

The design team prepared to conduct the main field-test from August 2013December 2013 in four schools across the district. We used data from our preliminary field-testing to incorporate many aspects of the key informants' feedback, as well as our own findings, into a now-amended PLC handbook. The design team verified that it had taken the correct approach by emphasizing the clarification of key PLC terminology, a user-friendly format, and outcome-based activities. After the preliminary field-testing took place, the design team highlighted two significant findings during their sessions together:

1. At step five of the R\&D process (main product revision), a roadmap for principals would need to be created to accompany the PLC handbook.

2. The roadmap would need to include a timeline that indicated when the PLC handbook activities would be presented to staff during the main field-testing. Step four of the R\&D process greatly informed the work of the PLC handbook design team as it began its main product revision.

\section{Step Five: Main Product Revision}

Step five of the R\&D cycle used the evaluation of the preliminary field-testing to improve the product. Feedback from key informants was useful in revising the product's 
content, sequence, and materials. In preparing a new draft of the handbook, the design team wanted to respond to questions and challenges that were discovered during the preliminary field-testing. We used our experiences with the first edition of the handbook to make several revisions to prepare the Estacada PLC handbook that would be used in the main field-testing. One of the most significant changes was the addition of a roadmap that would be used by principals to guide PLC work using the handbook. The roadmap included a timeline for when district-wide activities were to be completed as part of the main field-test in the fall; the roadmap created in step five of the R\&D cycle (main product revision) is included as Table 8 .

\section{Table 8}

Roadmap for the Estacada PLC Handbook Main Field-Test

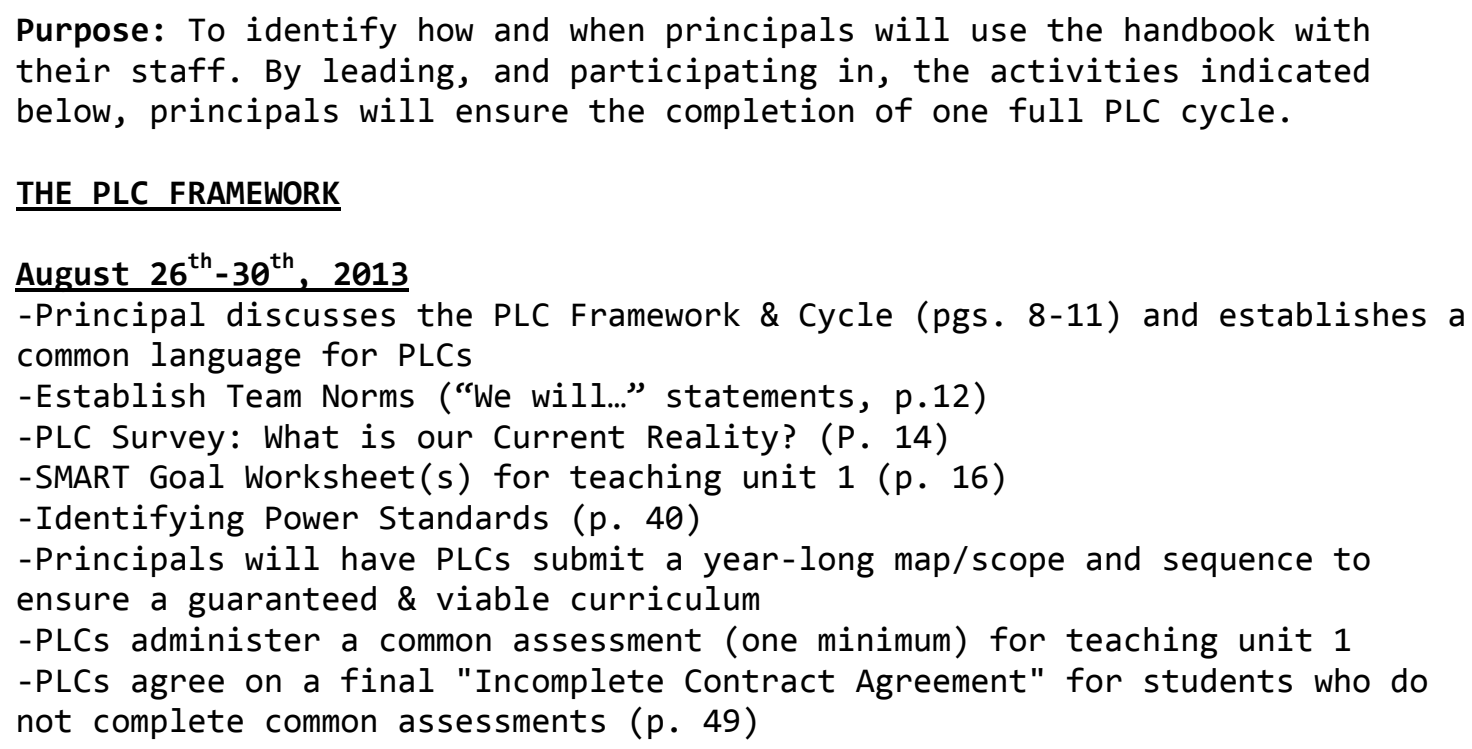


Table 8 (continued)

\section{CONTENT \& CURRICULUM}

September $3^{\text {rd }}-6^{\text {th }}, 2013$

-Complete Activity 4.0-"PLC Weekly Meeting Minutes Form"

-Complete Activity 5.0-"PLC Tasks"

-Begin Weekly PLC Meeting Minutes (every Friday) ( $p .18$ )

-Principal shares clarifies "PLC Tasks" with staff ( $p .20)$

COLLECTING \& ANALYZING DATA

Friday, September $20^{\text {th }}, 2013$

-Unit 1 assessment complete

-Principal has PLCS fill out reflection form ( $p .22$ )

\section{SUPPORTING SCHOOL CHANGE INITIATIVES}

Friday, September $27^{\text {th }}, 2013$

-Principal discusses Differentiated Instruction and Sheltered Instruction (DI/SI) with staff (p. 23)

-Review DI framework (p. 24)

-Review SI framework (p. 25)

-Activity 7.0-Principal asks PLCs to review Bloom's Taxonomy Question

starters and incorporate into lesson plans ( $p .27$ )

September $30^{\text {th }}$-October $4^{\text {th }}, 2013$

-Principal-selected coach completes one round of peer coaching using DI/SI walkthrough tool (p. 29)

-Principal discusses RTI and PBIS with staff (p. 30) and reviews RTI \& PBIS

frameworks (p. 31)

-Principal asks PLCs to complete a Pyramid of Interventions (for both

academics \& behavior ( $p .35$ ), and then review with the whole group

PLANNING FUTURE ACTION

Friday, October $11^{\text {th }}, 2013$

Activities 10.0 \& 11.0-PLCs complete first monthly academic \& behavior check sheets (pgs. $37 \& 38$, repeat monthly)

Friday, October $18^{\text {th }}, 2013$

-Principal discusses Proficiency-Based Learning with staff (pgs. 41-42)

-Principal has staff complete Analyzing Your Common Assessment worksheet using their unit 1 assessment ( $p .43$ )

-Activity 14.0-Principal has staff complete the "Creating Student-Friendly Rubrics” form ( $p .45$, repeat monthly)

-Principal has PLCs create (or adapt) a student-friendly rubric for their unit 2 assessment

*Repeat Cycle 
Table 8 (continued)

\section{TROUBLESHOOTING CHALLENGES}

Data collection to evaluate the handbook's effectiveness

-Feedback from the Design Team (Principals \& Vice Principal)-the Critical Friends Protocol will be used during Administrative PLC Design Team meetings (June, July, August, Sept, and Oct) (minutes will be recorded)

-Weekly Administrative PLC meetings (May 2013-Dec 2013) (minutes will be recorded)

-Weekly Teacher PLC minutes will be collected and reviewed by Principals (August-December 2013) (Principals will bring these minutes to Design Team meetings)

-Principals will share stellar examples of PLC work with each other and with their staffs

-Pre/Post survey data collected by principals (in August 2013 \& Dec 2013) as to the PLC handbook's effectiveness

-Pre/Post Survey of PLC teachers (in August 2013 \& Dec 2013) as to the PLC handbook's effectiveness

-At least one round of the cycle will be completed by December 2013, allowing the principals and PLC teachers to speak to the handbook's effectiveness

The addition of the roadmap gave principals better direction regarding how to lead and carry out the main field-testing of the PLC handbook. A number of other adaptations and edits were made during the main product revision. Significant edits were made to the introduction of the handbook, which included describing who should read the handbook, as well as a section on how the handbook should be used. The edits to the introduction of the handbook made it more inclusive and available to a wider audience of users in Estacada. From the data analysis conducted during preliminary field-testing, the design team uncovered the following 14 findings that needed revision at step five (main 
product revision). The findings from preliminary-field testing led to the improvement of the product during the main product revision. In preparation for the main field-testing of the PLC handbook, the following 14 findings were addressed:

1. In general, the graphic organizers used in the handbook needed to be revised to make the intended messages more clear. An example of a revised graphic organizer is the addition of "instruction" to the PLC cycle graphic (p. 11 of handbook) to make it clear that instructing students is a critical component that teachers working in PLCs engage in and work to improve.

2. Additional artwork needed to be added to the handbook to make it more aesthetically pleasing and appealing to the reader. An example of additional artwork includes the inclusion of more color photographs of real students and teachers working in a school environment; this helped attach more meaning and relevancy to many of the key concepts.

3. The PLC Survey "What is our Current Reality?" (p. 17) needed to be revised to ask more pointed questions of PLCs regarding how they complete their work. For example, the design team felt the need to include more detailed questions that dealt with the main school change initiatives of the district.

4. The SMART Goal Worksheet (p. 19) needed to be revised so that it would be applicable for a wider variety of educators, including classroom teachers and specialists. The design team also felt the need to more clearly define the fact

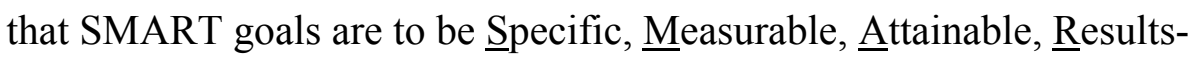
oriented, and Time-bound. 
5. The PLC Weekly Minutes Form (p. 21) needed to be revised so that it allowed for differences between the needs of elementary and secondary teachers and specialists.

6. The handbook needed to be more explicit in explaining the "PLC Tasks" outline (p. 23). The outline provided suggested due dates for the completion of tasks, but those due dates could be adjusted, at the principal's discretion, to fit the unique needs of each school.

7. A table (Table 1.0) needed to be added to more clearly define Estacada's current school change initiatives (p. 26). This would be helpful for handbook users new to PLCs as well as experienced PLC members who needed review.

8. A table (Table 2.0) needed to be added to more clearly explain the guiding principles behind Estacada's current school change initiatives (p. 27). This would be helpful for handbook users new to PLCs as well as experienced PLC members who needed review.

9. The handbook section that discussed DI/SI (pp. 28-30) needed to include graphic organizers that highlighted Tomlinson's (2001) Differentiated Classroom Instructional Framework and the Echevarria et al. (2010) Sheltered Classroom Instructional Framework to make content more comprehensible for handbook users.

10. The "Bloom's Taxonomy Question Starters" activity (p. 32) needed to be modified to include the revised version of Bloom's Taxonomy. The original taxonomy was developed by Bloom et al. in 1956. A revised model was 
developed in the 1990 s to better fit educational practices of the $21^{\text {st }}$ century. The revised Bloom's Taxonomy has been used in learning activities throughout Estacada, and has been promoted by well-respected professional developer Linda Vanderford (2012); therefore, this modification made sense.

11. Figures (Figures 4.0 and 5.0) needed to be added to make the essential components of RTI and PBIS more understandable for handbook users ( $\mathrm{p}$. 36).

12. The handbook section that discussed RTI/PBIS (pp. 35-43) needed to be revised to include information noting that many districts are now referring to the combined school change initiatives (RTI/PBIS) as EBISS; this would indicate to handbook users that Estacada was likely to move in this direction in the future.

13. For the "Creating Student-Friendly Rubrics" activity (p. 50), an example of a high quality rubric needed to be included to give handbook users more guidance in completing this activity.

14. Revision was needed in the description of the "Incomplete Contract Agreement" activity (p. 53) to include a statement that adjustments should be made by each PLC so that their "Incomplete Contract Agreement" with students was age-appropriate; this revision accounted for the unique differences between elementary and secondary students.

The findings addressed during the main product revision allowed the design team to make adjustments and refinements to the PLC handbook. Design team members felt 
that applying what they learned at step five of the R\&D cycle made for a better product, worthy of presentation to staff as a handbook to be used in a systematic way districtwide; this set the stage for the main field-test.

\section{Step Six: Main Field-Testing}

The main field-test allowed the design team to determine if the handbook was effective in guiding the work of PLCs in the Estacada School District. A secondary purpose of step six was to collect additional data to guide future handbook revisions. The design team received a waiver from the Human Subjects Research Review Committee at Portland State University to complete the rest of this study since no risk was posed to the design team members or their staffs. The goal of this problem-based R\&D project was to develop and evaluate an educational product-the PLC handbook. During the main fieldtest, the design team wanted to understand the strengths and weaknesses of the PLC handbook more deeply. We wanted to evaluate whether all of the essential concepts were present and well explained. We also wanted to know if the end users learned something from the systematic, district-wide use of the handbook. Moreover, we wanted to know if the handbook allowed users to be more efficient and productive in their PLC work.

The design team continued to meet weekly during the main field-test. The team also continued to participate in design team sessions, which featured use of the Critical Friends Consultancy Protocol and the adapted Evanson rubric to evaluate the effectiveness of the handbook, and the design team's group process. The methodical use of the protocol and rubric allowed the design team to gather and analyze data collected during the main field-test to further improve the PLC handbook. Design team members 
also continued to meet with their key informants (teacher leaders at their schools), and discussed how the PLC handbook had evolved and could be improved. Discussions with key informants took place on a regular basis, both in person and via e-mail. The design team members kept their own PLC handbooks in three-ring binders so that they could easily add, subtract, and modify pages.

The activities from the handbook were not given to staff members as a bound handbook; rather, design team members presented the activities to staff as worksheets or packets. The decision to present the activities in chunks was made deliberately so as to not overwhelm PLCs. In addition, the roadmap created for principals to use with the handbook indicated that certain activities were to be covered at certain points in time; therefore, presenting the activities in chunks made sense. Also, the cost of providing all staff members with bound handbooks was a factor, particularly since we anticipated making more revisions to the handbook during, and after, the main field-test.

During the main field-test, numerous adjustments were made in following the handbook, as well in following the timeline that was laid out in the principals' roadmap. The main field-test produced the following revisions made by the design team to improve the handbook:

1. It took longer for PLCs to create their SMART goals than originally expected. Oregon recently adopted Senate Bill 290, which was mandated to go into effect on July 1, 2013 (Oregon Department of Education, 2014a). Senate Bill 290, also known as the Educator Effectiveness framework, called for a more rigid form of SMART goals than teachers were used to. The Educator 
Effectiveness framework contained many new requirements that teachers were not familiar with, including linking their SMART goals to their students' tests scores on state assessments. While the new format for SMART goals under the Educator Effectiveness framework generated a variety of questions and concerns, teachers at all four schools were still able to complete their SMART goals during the main field-test; it did, however, take approximately three weeks longer than what the PLC handbook roadmap called for. The design team made adjustments to its SMART goal worksheets so that they would be more in line with the demands of the Educator Effectiveness framework.

2. With teachers taking longer to create their SMART goals, as noted above, PLCs administered their first common assessment with students in midOctober 2013, as opposed to mid-September 2013 (as proposed in the original PLC handbook roadmap).

3. Teachers were mandated to provide instruction for students using the new CCSS in 2013-2014. However, students would still be taking the OAKS as their state assessments in 2013-2014; OAKS is based on the old Oregon State Standards. Students will be mandated to take the SMARTER Balanced assessments as their state assessments in 2014-2015; SMARTER Balanced assessments are based on the CCSS. Needless to say, this transitional year produced a great deal of confusion and frustration for educators. New terminology was being learned during the main field-test by design team members through trainings that focused on the CCSS and the SMARTER 
Balanced assessments. One example of this new terminology included having "priority" standards-standards that would be more heavily weighted on the SMARTER Balanced assessments. The idea of "priority" standards was similar to that of "power" standards, which were tested on OAKS. The term "power" standards was included in the PLC handbook; this term was clarified and changed to "priority" standards.

In general, most of the suggested due dates indicated in the timeline of the PLC handbook roadmap were pushed back by approximately three weeks. Aside from the PLC activities taking longer than expected, and the issues encountered above, the main field-test moved forward as planned. PLC handbook activities were presented by design team members, and the activities were used in all four schools.

During the main field-test, the design team was surprised by the number of parents, families, and community members who started showing an interest in the PLC process. Parent leaders, in particular, voiced their desire to learn more about PLCs, and how they could take part and contribute. In their discussions during design team sessions, principals noted that there is usually a core group of parents at each school who are actively involved. These core parent leaders hoped to become more involved in the school improvement process-beyond the traditional ways parent volunteers have helped in the past (i.e., organizing fundraisers, running copies for teachers, and helping out in classrooms).

Site council meetings are held at each school on a monthly basis to discuss issues relative to school improvement; the members of these meetings include both educators 
and parents. Site councils evolved into active PLCs during the main field-test, and had significant influence throughout each school. Site council meeting minutes provided the design team with important data that was analyzed and coded for themes during their sessions together. From site council meetings, and general observations as to how site councils were positively impacting schools, it became clear to the design team that future revisions to the PLC handbook should include activities that make the handbook more accessible to a broader audience. The team wanted to show parents, families, and community members how they could become involved in the PLC process.

The design team was excited to formalize ways for parents, families, and community members to become involved in PLCs. In the field-tested handbook, the activities (1-15) were specifically designed for educators to use in the school setting. A finding from the main field-test was that parent/family/community involvement with PLCs was a missing element. The design team felt it could create additional activities (which would eventually become activities 16-22) that appealed to both educators and parents/families/community members. The additional activities for the handbook would be based on projects that were emerging around the district, as well as from trainings that design team members (and other educators) were participating in with parents, families, and community members.

After the conclusion of the main field-testing of the PLC handbook, the design team noted that the handbook could be improved by including activities that help build consensus; this is an area that requires more R\&D. Activities relative to building consensus are now included in the handbook, but they still require field-testing. The 
additional activities were greatly informed by the design team's communication with parent, family, and community leaders. The hope from the design team was that the activities focused on building consensus could assist PLCs, parents, families, and community members as they looked to make difficult decisions. Chenoweth and Everhart (2002) affirmed that in order to build consensus, a leadership team (the design team in this case) will need to be genuinely committed, honest, and open about potential benefits and problems, and let others freely choose to become involved. As mentioned previously, Estacada faces some significant challenges, and difficult decisions will need to be made in the future; consensus-building activities can help Estacada make these tough decisions. The amended handbook now attempts to help PLCs work collaboratively with various local district and community stakeholders; this collaborative work is necessary to take future action in our small, rural school district.

In addition to involving parents, families, and community members more deliberately in PLCs, the design team also realized the need to facilitate better communication with its district and community stakeholders. The team wanted a broader audience to know what PLCs were working on and achieving. Reflections and comments from design team sessions indicated that since parents, families, and community members had such a strong voice in the future direction of our district, we wanted them to be more informed about important school change initiatives, and how PLCs were working to address them. We wanted to communicate more effectively with our district and community stakeholders in a variety of ways, including: 
1. Making the PLC handbook more accessible to a broad audience (including parents, families, and community members)

2. Creating a monthly district newsletter-News from the 108-that would highlight the accomplishments and efforts of PLCs (and how readers of the newsletter could become involved)

3. Create a district Twitter feed that would provide a broad audience with realtime updates regarding PLC accomplishments and efforts (and how readers of the Twitter feed could become involved)

The fact that parents, families, and community members called for increased involvement (and increased communication) regarding PLCs was a significant finding for the design team at step six of the R\&D cycle. This significant finding was addressed heavily in step seven (operational product revision), as we added the additional activities (16-22) to the handbook.

The main field-test of the handbook indicated to the design team that additional sections to the handbook would be needed to help engage parents, families, and community members in PLC work. However, the team also wanted to understand how effective the original handbook activities (1-15) were for educators, and how they could be improved. At the conclusion of the main field-test, the design team followed up with a 10 question anonymous online survey, which was completed by design team members and their key informants. The questions included in the survey focused on strengths, weaknesses, content, scope, sequence, style, and tone of the handbook. To get quantitative data on the effectiveness of the handbook, the design team asked respondents 
to self-report on their knowledge and abilities. The results of the survey, which informed the operational product revision of the handbook, are included in Table 9.

\section{Table 9}

Survey of the Effectiveness of the Estacada PLC Handbook

\begin{tabular}{|l|l|l|l|l|l|l|}
\hline Question/Statement & $\begin{array}{l}\text { Strongly } \\
\text { Disagree }\end{array}$ & Disagree & Neutral & Agree & $\begin{array}{l}\text { Strongly } \\
\text { Agree }\end{array}$ & $\begin{array}{l}\text { Average } \\
\text { Rating }\end{array}$ \\
\hline $\begin{array}{l}\text { The handbook is well organized and } \\
\text { easy to follow }\end{array}$ & 0 & 0 & 0 & 6 & 4 & 4.40 \\
\hline $\begin{array}{l}\text { The handbook does a good job of } \\
\text { helping PLCs translate concepts into } \\
\text { concrete examples }\end{array}$ & 0 & 0 & 0 & 7 & 3 & 4.30 \\
\hline $\begin{array}{l}\text { The activities in the handbook are } \\
\text { clear }\end{array}$ & 0 & 0 & 0 & 7 & 3 & 4.30 \\
\hline $\begin{array}{l}\text { All of the essential components for } \\
\text { PLC work are present and well } \\
\text { explained }\end{array}$ & 0 & 0 & 1 & 5 & 4 & 4.30 \\
\hline $\begin{array}{l}\text { I learned something from reading the } \\
\text { handbook }\end{array}$ & 0 & 0 & 0 & 5 & 5 & 4.50 \\
\hline $\begin{array}{l}\text { The scope and sequence of the } \\
\text { handbook is appropriate }\end{array}$ & 0 & 1 & 1 & 4 & 4 & 4.10 \\
\hline $\begin{array}{l}\text { The style of the handbook is } \\
\text { appealing }\end{array}$ & 0 & 0 & 2 & 4 & 4 & 4.20 \\
\hline The tone of the handbook is collegial & 0 & 0 & 1 & 1 & 8 & 4.70 \\
\hline $\begin{array}{l}\text { The handbook reflects the unique } \\
\text { needs of our small, rural district \& } \\
\text { community of Estacada }\end{array}$ & 0 & 0 & 1 & 4 & 5 & 4.40 \\
\hline \begin{tabular}{l} 
The handbook is a useful tool \\
\hline
\end{tabular} & 0 & 0 & 0 & 5 & 5 & 4.50 \\
\hline
\end{tabular}

Space was also included in the survey for respondents to provide comments. The comments acquired from the survey included: 
"I appreciated having the activities already written out for us."

"The step-by-step process described in the handbook proved to be very helpful."

"Good reminders and clarification of the components of collaboration."

"Excellent visuals."

"I can see this being a tool I return to regularly to not only guide me in my PLC, but to answer many other components mentioned in the handbook."

"Generally the style of the handbook is appealing, but the fonts could be more consistent."

"Still needs some refining to reflect our small, rural district and community."

"The handbook is useful and will only get better."

"Perhaps use less ClipArt and more real pictures."

"This is a great tool for those in the early stages of development, and as a reminder for those who have lost some fidelity to the PLC process."

"I believe this handbook would work for other districts with a desire to promote more collegiality and community within their staff."

Design team members and key informants were willing to ask good questions and provide useful feedback related to the PLC handbook used by the district. This group of respondents affirmed that the revisions made as a result of the preliminary field-test were effective, but also pointed out aspects related to the handbook's clarity and usability that were missed prior to the main field-test. After the main field-test, design team members noted that the feedback from key informants used in this study may be atypical. We selected people who frequently led, or actively participated in, staff development activities related to PLCs. The members of this group are recognized by their peers to be excellent educators, and have displayed an above average eagerness to learn and promote 
new methods. The quality of the key informants' feedback and specific comments may not reflect the viewpoints of the average staff member. However, to complete this problem-based $R \& D$ project, the design team needed to rely on those who displayed enthusiasm and the ability to think critically about PLCs.

As the design team came to the end of the main field-testing of the handbook, it became more convinced of the power and benefits of schools working in PLCs. The PLC handbook, while still in need of improvement, received high ratings overall; the data revealed it to be a useful educational tool in Estacada. In fact, going through the R\&D cycle prompted the design team to recommend a new school configuration to the school board chairman in December 2013 that it believed would help address the current imbalanced school populations and strengthen PLC work. Through evaluation of the handbook, the design team felt that it was an effective tool for our district to use in its current configuration, but that it could be even more effective if PLC groupings were organized in a way that could maximize the potential for teamwork. The design team's recommendation for reconfiguration of the district's schools (which, from the design team's perspective, would also make the handbook more powerful) included:

- One K-2 elementary school

- One 3-5 elementary school

- One 6-8 middle school

- One 9-12 high school

This configuration would allow all common, grade-level specific PLCs from across the district to come together and join forces under one roof to more efficiently and 
productively address student needs and school change initiatives through use of the handbook's activities.

\section{Step Seven: Operational Product Revision}

The operational product revision step used data obtained in the main field-test to prepare the product for use by a broader audience, including parents, families, and community members (Lorenz \& Pichert, 1989). We used the second main product edition of the handbook and analysis of the feedback gathered during step six (as described above) to make several major revisions to prepare the operational handbook for use in a small, rural district. Specifically, the design team wanted to improve the handbook for optimal use in Estacada. Other districts similar to Estacada, in terms of size and demographics, could benefit from replicating this study or they could effectively use the Estacada PLC handbook to guide their collaborative work.

The main field-test affirmed for the design team that heavy use of the theoretical concepts behind PLCs, and the related initiatives, would be off-putting to users. Therefore, basic clarification and outlining of the PLC process, and associated initiatives and activities, would be most useful for practitioners. They were most interested in activities and resources that were easily understood and would help them complete their work. A user-friendly format would also make the handbook more accessible for parents, families, and the community. The relationship, roles, and responsibilities of the district and community in the PLC process needed to be clarified. Parents, families, and community members can play a more active role in PLCs in Estacada. 
The R\&D process of field-testing was valuable in producing a high quality handbook with instructional materials for a general audience. Estacada would benefit from continued field-testing and revising of the handbook to show parents, families, and the community how they could become more involved in PLCs. The design team experienced success in creating a PLC handbook for educators using the step seven R\&D cycle, but there is still so much more that we need to learn.

Design team members participated in two trainings that helped inform their perspective on parent, family, and community involvement in PLCs. Incidentally, both of these well-structured and informative trainings included the use of a handbook. Design team members reviewed, and participated in, activities from these proven handbooks, which greatly informed the further development and design of the PLC handbook. The two trainings the design team members participated in included:

1. Family Involvement Matters-facilitated jointly by the Oregon Department of Education, Education Northwest, and Portland State University

2. Ford Leadership Institute-facilitated by the Ford Family Foundation and Rural Development Initiatives

These multi-session trainings-attended by Estacada design team members, educators, parents, families, and community members-provided the design team with ideas for activities to include in the PLC handbook that would encourage parent, family, and community involvement in PLCs in our small, rural school district. In design team sessions, principals noted that parents, families, and community members in a small, rural district (like Estacada) have a strong, prevalent voice regarding school improvement 
issues. In larger school districts, there are several layers of bureaucracy that parents, families, and community members must navigate through before reaching key district decision-makers; this is not the case in Estacada-parents, families, and community members have direct access to key educational decision makers. While increased parent/family/community involvement is desirable in Estacada, it has often led to complexity in building consensus for decisions; the design team wanted to address this challenge in the additional sections of the handbook.

Adding sections to the handbook for family and community involvement will allow an academic R\&D process to unfold that could help parents, families, and community members define, refine, understand, and improve its practices and model effective techniques for professional dialogue and engagement. The main revisions made to the handbook after main field-testing included:

- A graphic organizer was added (p. 55 of the handbook) that illustrated the common interests of the Estacada School District and the community of Estacada. This graphic organizer highlighted the mutually-beneficial PLC projects that parents, families, and community members could become more involved with alongside educators. This graphic organizer set the stage for the additional activities of the handbook that were geared to address parent, family, and community involvement.

- Activity 16 was added (p. 57) to show how parents, families, and community members could become involved in grant writing to help support PLCs. 
- Activity 17 was added (pp. 58-59) to help PLCs, parents, families, and community members build consensus around difficult decisions.

- Activity 18 (p. 60) was added to show parents, families, and community members how they could help support PLCs as they worked to provide after school enrichment activities for students.

- Activity 19 (pp. 61-65) was added to show parents, families, and community members how they could assist PLCs in their P-3 alignment efforts. P-3 alignment involves collaboration with local preschools, childcare providers, and parents to ensure that students are kindergarten-ready and reading at grade level by third grade.

- Activity 20 was added (pp. 66-67) to show parents, families, and community members how they could be involved with helping PLCs move forward in their use of instructional technology to assist students and increase PLCs' efficiency and productivity.

- Activity 21 was added (p. 69) to show parents, families, and community members how they could help PLCs secure financial assistance through fiscal partner relationships with local nonprofit agencies.

- Activity 22 was added (pp. 71-74) to show parents, families, and community members how they could access new methods of communication about PLC efforts from the district (including the district's newsletter-News from the 108 and the district's Twitter feed). 
The proposal of the reconfiguration option recommended by the design team $(\mathrm{K}-$ 2, 3-5, 6-8, and 9-12) was met with some resistance by parents, families, and community members. A school board-initiated online survey was released in the winter of 2013. This survey touched on a variety of school issues, but primarily focused on soliciting input from the community regarding school reconfiguration options. The survey generated nearly 700 responses; and the responses were varied. There was not a clear preferred choice when it came to school reconfiguration. This school board-initiated survey provided the design team with valuable data that greatly informed the design team session discussions.

While the reconfiguration option recommended by the design team was not rated low, there were enough varied responses to give the design team pause. In addition, the fact that a new superintendent would be hired on July 1, 2014, prompted the design team to wait until more research could be conducted. We felt the need to incorporate the perspective of our new district leader into our vision for PLCs. The complexity of building consensus around school reconfiguration affirmed the design team's assumption that further R\&D was needed regarding how to involve parents, families, and community members in PLCs. The data analysis completed by the design team at step six (main field-testing) greatly informed the work completed during operational product revision.

\section{Chapter Summary and Reflection}

Guiding the Work of Professional Learning Communities: Perspectives for School Leaders was designed and developed to move the Estacada School District forward in its use of PLCs. The handbook was developed using the R\&D process, which included an 
extensive literature review, preliminary and main field-testing for validation, and revisions. The handbook was piloted in the Estacada School District for the main fieldtest and operational product revision. The product was reviewed by experts in the field, including principals and teacher leaders, who provided formative evaluation of the handbook's content, format, and practicality. All suggestions, notes, and reviewer comments were considered for improvement of the handbook. The piloting schools' principals provided a wealth of information on the handbook based on their experience participating in the main field-test. The principals used the handbook to guide their staffs through professional development. The handbook design team used a protocol and rubric for sharing and evaluating the pilot schools' experiences while testing the product. The educational R\&D process resulted in the development and improvement of a school leader's handbook ready for dissemination and implementation in a school district setting.

Bridges and Hallinger (1995) described the problem-based Ed.D. dissertation as providing an opportunity for students to demonstrate their ability to apply knowledge in a professionally productive and academically sound manner and as a vehicle for building meaningful connections between research, theory, and practice in schools. As the lead member of the design team, I considered it an honor to work with such dedicated professionals. As I complete this project, I can say that Bridges was correct in his assessment of PBL project implementation. This project has been professionally productive. The PBL project stretched me academically, and has led to a deeper understanding of how to use research and theory to examine my own practices, as well as 
the practices of others. I have grown into a more thoughtful and resourceful school leader. I have become more academic, a better researcher, and have learned how to produce a high quality handbook that has allowed my school district to move forward in its PLC work in a positive and productive manner.

Through completing the dissertation process over the past 4 years, I have gained a reputation as an expert in the area of PLCs. Being an expert in the area of PLCs has led to collaborative efforts with many educators. As mentioned in chapter two, the principal of a school fills a variety of roles, many of which are closely tied to family and community engagement. I am grateful for developing a deeper understanding of family and community engagement through my experience as a principal. Estacada now has the practical know-how to create a PLC handbook that assists school leaders in creating a positive and sustainable change to benefit students, the district, and the community. In Estacada, anyone can be part of a PLC; not just educators. The work involved with broadening PLCs in Estacada will be a part of the design team's next steps.

Final product revisions need to include newly developed and field-tested PLC activities that better engage parents, families, and community members. PLC activities that are welcoming, accessible, and useful will appeal to parents; and their involvement will help create situations that benefit students, and the community as a whole. Estacada has more work to do, and the design team that created the Estacada PLC handbook plans to be a large part of those future plans and final product revision. Future collaboration through PLCs has great promise in Estacada. 
Based on the data collected at Estacada's four schools during their time piloting Guiding the Work of Professional Learning Communities: Perspectives for School Leaders, design team members noted that additional R\&D was needed relative to family and community involvement. Parents and community members in a small, rural school district typically have more of a voice than those in larger districts with more barriers and layers of bureaucracy. During the main field-test, we learned that parent and community interest in the PLC handbook was high. However, at the time, we did not have activities in the handbook that created a means for parents, families, and communities to actually become involved in the PLC process. Consequently, activities encouraging parent/family/community engagement through PLCs in Estacada were added to the handbook, but now they will require more exploration and refinement. Field-testing is needed for the activities that were added to the handbook after the main field-test; this future field-testing will further inform the design team's R\&D process. 


\section{CHAPTER 5}

\section{CONCLUSIONS, SPECULATIONS, AND RECOMMENDATIONS FOR LEADERSHIP}

\section{Introduction}

This chapter summarizes the R\&D of a PBL tool-the handbook, Guiding the Work of Professional Learning Communities: Perspectives for School Leaders. Specifically, the chapter discusses the overall assessment of the research experience, and reveals the results of the operational product revision. Speculation about further development and use of the handbook is also included. The chapter concludes with implications, suggestions, and strategies for dissemination and use of the handbook, as well as recommendations for future study. Finally, the chapter offers recommendations for school and district leadership for facilitating the work of PLCs.

The idea for developing the Estacada PLC handbook was established through an extensive literature review that began in the summer of 2010. The relevance of the problem was revealed through discussions with interested parties ranging from principals, central office administrators, and superintendents of districts to Estacada students, staff, parents, families, and community members. A prototype of the product was developed prior to the 2013-2014 school year, and main field-testing began in August 2013 in Estacada schools. The product was revised and refined based on preliminary and main field-testing findings. The main field-test was conducted at Eagle Creek Elementary, Clackamas River Elementary, Estacada Junior High, and Estacada High School and 
concluded in December 2013. Overall, the handbook received positive feedback, and was judged to be useful. Future revisions to the PLC handbook will occur, as the design team continues to improve and develop the product.

The PLC handbook was an educational product that filled a demonstrated need in the Estacada School District. The handbook allowed the district to engage in a systematic process for problem solving and helped create a culture of continuous improvement. Specifically, the handbook allowed the district to exhibit the four main characteristics of schools as learning organizations: adaptability, focus on variation, multiple connection channels, and continual review of process and content (Chenoweth \& Everhart, 2002). Estacada's use of the PLC handbook allowed the district to exhibit the following characteristics:

- Adaptability-Schools were able to make timely changes in curriculum and instructional strategies to meet the needs of students. The PLC handbook activities were made to be adaptable to fit the unique needs and culture of each school in the district.

- Focus on variation-Schools were not trapped by routine, but rather PLCs differentiated teaching and learning practices based on student needs.

- Multiple communication channels-Information flowed up, down, and sideways rather than just from the top down. Schools cultivated leadership from a variety of district and community stakeholders including principals, teachers, support staff, parents, families, and community members. 
- Continual review of process and content-The PLC cycle was repeated and activities from the handbook were continually revisited. Schools now believe in the power of information and data to improve the end result of each school.

\section{Overall Conclusions and Assessment of the Experience}

The purpose of this study was to create and field-test a handbook to guide school leaders in the implementation of the PLC process. The goal was to ensure that PLC work was focused, systematic, efficient, and productive, and that it led to a positive school and district culture. The following research objectives were achieved:

1. The initial steps of the $R \& D$ process revealed there was a lack of systematic information across the Estacada School District with regard to how principals and other school leaders should work in PLCs to promote school and district improvement; the PLC handbook filled this void.

2. The literature review conducted by the design team supported the belief that working in PLCs can positively impact many aspects of the school experience. For example, the PLC process influences conversations about the importance of professional development, the value of quality instruction, and the promise of assuring that all students can learn (Peterson \& Deal, 1999). Chenoweth and Everhart (2002) reported that schools serious about creating significant changes in teaching and learning that will positively impact student achievement must go through the difficult process of school change. School change is defined as challenging current practices, patterns, and norms by examining them, and implementing change when it is appropriate to ensure 
the success of all students. Working in PLCs, guided by the handbook, has facilitated the Estacada School District's change process.

3. A PLC handbook to be used by school leaders was developed using the R\&D cycle. The design team was able to evaluate and refine the handbook to assist school leaders in making successful decisions about what is best for the school and district moving forward.

4. The handbook for school leaders guiding the PLC process was further developed by the design team through preliminary and main field-testing, which laid the groundwork for future revision, refinement, and development.

As the design team leader, and primary author of the handbook, I have learned a great deal by going through this process. When I began studying PLCs as a teacher, I felt it was a significant topic to explore; my 9 years of experience as a building principal have confirmed this belief. During my tenure in Estacada, I have had the opportunity to not only gain experience as a principal guiding PLCs, but I have also served as a director of district programs at the central office, and have served as the superintendent's designee. In assessing my experience, I would say unequivocally that I have grown as a researcher as well as an educational leader. Through this experience, the design team has become more in tune with the strengths and challenges of implementing PLCs in their schools. Principals have learned to listen to the concerns of stakeholders who make up the school, district, and community and to take the time to understand their concerns more deeply. When dealing with change, collaborative leadership, and school culture, we now rely on practitioner experiences coupled with systematic research. Through this 
process, we have learned to look at situations as a whole, and identify factors within the whole that stand out. We have also become more adept at understanding and appreciating a variety of perspectives from stakeholders with diverse backgrounds and needs. We have learned to lead using a social justice lens (i.e., working to ensure success for all students regardless of their background or challenges) to make PLCs work more meaningful and accessible to all school, district, and community stakeholders.

As school and district leaders, the design team has made reflective practice a part of our schools' normal routines. As a district, we spend time assessing what is working, and what needs to be changed to best meet the needs of our students. The design team has realized that looking for a "quick fix" is rarely the answer to complex issues and educational needs. Design team members meet with a variety of PLCs on a weekly basis, and we discuss problems, analyze them, and brainstorm possible solutions. When we find a solution that may work, we field-test it through planned strategies, and then assess the effectiveness of the outcomes using protocols and rubrics to ensure that we are following a systematic problem-solving process. All of the design team's educational leadership experiences have helped us grow more confident in our ability to speculate about future use of PLCs in our district.

\section{Speculations About Future Research, Development, and Use of the Product}

This dissertation will have an afterlife in Estacada. The handbook that was originally field-tested from August 2013-December 2013 included 15 activities. Numerous adjustments and refinements have been made to activities 1-15 of the handbook. Since December 2013, seven additional activities have been created. The 
additional activities include opportunities for parent/family/community engagement through PLCs. Activities 16-22 will be field-tested throughout the remainder of the 20132014 school year, as well as over the next several years. The new PLC activities included in the handbook focus of the following topics:

1. Grant writing

2. Building consensus

3. After school activities

4. P-3 alignment

5. Instructional technology

6. Communication and public relations

PLC activities that promote grant writing will benefit the district and the community. The now-amended handbook includes a link to the online Oregon Rural Communities Explorer tool (Natural Resources Digital, 2014) this tool allows local grant writers to access data relevant to the community that is needed to complete grant proposals. The information available from the online tool includes community data, rural research, community stories, local reports contributed by residents, historical documents, community visions, and additional resources and portals. Also included in the nowamended PLC handbook, is a sample Memorandum of Understanding for a fiscal partner relationship. The sample Memorandum of Understanding was included to encourage PLCs to partner with local community nonprofit (501c3) organizations to pursue mutually beneficial grants for the district and community. Grant writing to support PLCs 
is an area that needs to be more fully explored by educators, parents, families, and community members.

Building consensus is another skill that needs to be further developed in Estacada. When the district faces difficult decisions (decisions around school reconfiguration, for example), it needs high quality tools and activities that lead participants to accepted outcomes. The design team realizes that it is unlikely to arrive at a unanimous decision when soliciting input from a wide variety of stakeholders, including educators, parents, families, and community members. However, the district needs to approach its difficult decisions in a systematic way so that all stakeholders have relevant information, the ability to provide their input, and a solid rationale for how and why final decisions are made. Estacada is growing in the area of building consensus, but more work needs to be done.

Another area related to PLC collaboration that will be more fully explored in the future is establishing quality after school activities for students. In 2013-2014, the elementary schools of the district created an after school enrichment program; the program is currently in its pilot year. The after school program requires PLC collaboration among a variety of stakeholders, including teachers, parent and community volunteers, students, families, bus drivers, school kitchen staff, and school principals. More field-testing needs to occur so that those involved with the after school program can further develop and refine the program. The acronym for the after school enrichment program is STEAM (Science, Technology, Engineering, Arts, and Math), which is a reference to the subject areas that the program attempts to address. PLCs can help 
Estacada recruit more local volunteers who have enthusiasm, knowledge, and interest in STEAM topics so that they can share their expertise with students.

Another area requiring further development is the collaboration of efforts targeting the district's P-3 alignment strategies. P-3 alignment efforts are focused on prenatal through third grade activities (P-3). The goal of P-3 alignment is to have all children in the district kindergarten-ready and reading at grade level by third grade. P-3 alignment involves collaboration among a variety of people, ranging from elementary school teachers, staff, and principals to parent volunteers, families, social service agencies, preschools, and child care providers. Estacada needs to establish the conditions for a quality learning environment during early childhood; PLC work can help ensure that this happens. In the fall of 2013, collaboration among PLCs allowed the district to submit a P-3 alignment grant proposal for $\$ 225,000$ to the Oregon Community Foundation. If awarded the grant, it will be used over the course of the next 3 years to assist with P-3 alignment efforts in our community. The district is still waiting to find out if it has received the grant; whether or not the grant is received, this important work must continue. Future P-3 alignment plans are currently in development and ready for fieldtesting.

An area that requires further exploration is instructional technology. Instructional technology can greatly inform and assist PLCs in their work. PLC handbook activities that promote the use of instructional technology have been added, but still need refinement. The district's Technology Mini Grant Application was distributed in 2011 to encourage teachers to explore more creative and innovative strategies to use with their 
students, and among themselves in their PLC work. The Technology Mini Grant Application is now included in the PLC handbook so that it has optimal accessibility. Many PLCs are now using a "Bring Your Own Device" model in their meetings, and use technology to become more efficient in gathering and recording information, which streamlines their PLC work.

An Estacada PLC attended the instructional technology-themed International Association for K-12 Online Learning (iNACOL) conference in Orlando, Florida in October 2013. The conference attendees included design team members, teachers, and school board members from Estacada, as well as administrators, teachers and staff from Estacada's virtual charter schools. The core mission of the iNACOL is to ensure all students have access to a world-class education and quality blended and online learning opportunities that prepare them for a lifetime of success. The conference was a phenomenal opportunity to explore how the district can collaborate with its charter schools to identify and infuse quality instructional technology into our "brick and mortar" schools.

PLC activities related to instructional technology are currently underway, and will continue. For example, the third grade PLC at Clackamas River Elementary recently received a grant for $30 \mathrm{iPad}$ minis, which are now being used to help students become better readers. The first/second grade PLC at Eagle Creek Elementary is now piloting Dreambox, an adaptive math intervention program that students are accessing through iPads. Estacada High School has been piloting the Grade Tree program, which is used to provide immediate assessment feedback to students and parents through text messages 
and e-mail. There is a great deal of excitement about instructional technology in PLCs throughout the district. The district plans to form a PLC that will submit a grant proposal to the Next Generation Learning Challenge in 2014-2015 for a sizeable amount of money that would be spread over multiple years. The Next Generation grant proposal will be targeted to eventually achieve "one-to-one status" throughout the district (one educational device per student and teacher).

Related to technology, collaboration through PLCs has allowed the district to revamp the way it communicates and promotes itself with students, staff, parents, families and community members; this is another area, though, that requires further R\&D. The newest version of the PLC handbook now contains activities that promote better communication. For example, the handbook now includes step-by-step directions for following the district's official Twitter feed: @esd108 (created on 8/1/13). Twitter is an online tool that is now used by a variety of businesses, schools, districts, and news outlets. The appeal of Twitter is that it promotes messages that are short, sweet, and to the point; yet it also allows users to dig deeper and learn more, as "Tweets" can include pictures, links to other websites, and links to more detailed documents. PLCs in Estacada have been encouraged to create their own Twitter feeds; many have done so. When teachers facilitate noteworthy activities with students, they can document it via Twitter and share their news with a larger audience that includes fellow educators, parents, families, and community members.

Regarding the improvement of communication and public relations, the district also launched a monthly district newsletter called News from the 108 in September 2013, 
which publicizes and celebrates PLC success stories. Links to the district's monthly newsletter are provided on the district's Twitter feed, as well as on the district's website. The district currently has a PLC working on redesigning its district and school websites to make them more appealing, user-friendly, and accessible to district and community stakeholders. The improvement of communication and promotion of current PLC-related activities, as well as future activities, projects, and initiatives will continue.

Communication with district and community stakeholders is extremely critical in a small, rural school district like Estacada.

\section{Recommendations for Leadership}

Throughout this process, I have been continually surprised by the amount of information and positive feedback that has been shared with me around PLC and school change research. I received invitations from educators through email, phone calls, letters, and meetings to speak about the benefits of working in PLCs. Throughout this dissertation work, I have participated in statewide and national conferences as a participant, panelist, and educational consultant. For example, the Clackamas Educational Service District recruited me to be a moderator of collaborative group work aimed at helping teachers and PLCs gain a better understanding of CCSS. This dissertation has opened up a new world, full of opportunities and unlimited experiences. The district has already benefited from the tools and activities provided in the handbook; and more is to come. Estacada used the activities and methods in the handbook to guide them through their PLC work. Currently, staff, students, parents, families and community members in Estacada are feeling positive about collaborative planning and changes that have taken 
place due to the tools and activities in the handbook. The design team will continue to provide support for the district and community as we pursue mutually beneficial PLCrelated activities, grants, and projects.

Continued promotion of collaboration through PLCs may spark the publishing of educational articles, books, websites, or state and national presentations from Estacada PLCs. There are a number of grants currently available that target small, rural school districts and communities; leaders in Estacada plan to pursue these opportunities. Through PLCs, Estacada must continue to build capacity and cultivate local talent to pursue opportunities that bring more resources and staff to our district. Recommendations for district leadership include the following short-term goals:

- Present with the design team at the annual Confederation of Oregon School Administrators (COSA) conference in June 2014 to share our experience developing and field-testing the Estacada PLC handbook

- Continue to collaborate with PLCs affiliated with the Ford Leadership Institute to finish the work necessary for the completion of a $\$ 12,000$ auditorium revitalization grant that was recently awarded to our district/community

- Continue to inform the district and community of the benefits of PLC collaboration as we review school reconfiguration options

- Continue as an active member of Portland State University's Educational Administration doctoral cohort, to support colleagues as they work toward their dissertation completion 
Other recommendations for leadership include long-term goals:

- Publish an educational article or book on guiding the work of PLCs, particularly in a small, rural school district and community

- Extend and further develop PLC collaboration among the districts four traditional "brick and mortar" schools and the district's online charter schools, and eventually co-present at a future iNACOL annual conference

- Become an educational consultant to help guide the improvement of PLC work in schools and districts struggling with implementation, and help school leaders manage the change process

- Become more involved at the central office level of leadership, and eventually earn a position as a district superintendent

Following the first seven steps of the R\&D process has led to the development and refinement of an effective PLC handbook. In terms of recommendations for future leadership, if this product were to be taken to scale, the design team would complete the full R\&D process by completing the last three steps.

- Step 8: Operational Field-Testing-This step could be completed in a variety of ways, but the optimal opportunity would be for several schools and districts throughout Oregon, particularly from small, rural areas to pilot the handbook. The piloting schools and districts could then share the roadblocks and success stories pertinent to their use of the handbook, which would in turn support the design team in making a quality final product revision. 
- Step 9: Final Product Revision-This step would include the refinements, edits, and improvements that were made evident after analyzing the data from the operational field-test. At this step, the handbook would be professionally edited, and perhaps even reviewed by a graphic designer for improvement of the visual design and color schemes, which would create a more appealing layout than that created in the handbook used during main field-testing.

- Step 10: Dissemination and Implementation-This step consists of finding ways to make the product available to a wide audience. This could be done in a number of ways. For example, the handbook could be published by an Oregon Educational Service District, adopted by the Oregon Department of Education, or even published by a publishing firm. The design team could offer professional development workshops to provide support for guiding the work of PLCs through use of the handbook.

Full completion of the R\&D process, steps one through ten, as demonstrated by Borg and Gall's (1989) research has proven that the R\&D cycle is an effective means to develop, field-test, and refine educational products that are useful to school practitioners.

Research proves that certain leadership actions can be taken to ensure results (Reeves, 2006; Schmoker, 2006). A list of recommendations for leadership has been compiled, based on the design team's experience. As practicing school principals in the field, we feel this is a way to share helpful insights regarding collaboration through PLCs. Districts could replicate this study, use the data to inform the work of their PLCs, and 
provide research-based approaches for school leaders. Specific recommendations for PLC work from the design team are included below:

- Ensure rigorous goal and data-driven PLC activities, such as basing intervention and enrichment opportunities for students on results from common assessments. In order to make gains and breakthroughs using the PLC process, effective school leaders need to guarantee fidelity to the process, otherwise teachers will revert to bad habits.

- Have PLCs complete the work required of the four prevalent school district change initiatives. They include: SI/DI, RTI/PBIS, CCSS, and ProficiencyBased Learning (described in detail in chapter 2).

- Principals and school leaders should develop a set of effective PLC activities that all groups are working to advance; these activities should be organized in a comprehensive handbook.

- Ensure that PLC members are holding students accountable to similar expectations in each and every classroom by teaching to the same priority standards and checking for understanding through the use of common assessments.

- Stress the importance of studying meaningful student learning data as the foundation for all lesson planning, collaborative meetings, professional development, and meetings to plan intervention and enrichment opportunities.

- Build and manage a high functioning staff though PLC work, and encourage the emergence of new school leaders. For example, when teachers exhibit 
leadership skills, provide them with opportunities for expanded responsibilities within the PLC process.

- Become skilled at working in PLCs, and provide learning opportunities for school leaders to share their knowledge.

- Revisit PLC activities on a regular basis and provide PLC members with ongoing professional development and helpful tools to support their growth. Provide PLC members with direct feedback and job-embedded learning activities.

- Recruit, select, and evaluate school leaders based on their commitment to the PLC process. Publicly acknowledge and reward high performing school leaders for their PLC work.

Across the nation, increased accountability is the new norm. It requires a different kind of leadership, one that requires schools to better serve students. Sergiovanni (1995) affirmed that leadership development tends to be shaped by a set of beliefs, opinions, values, and attitudes, which provide a foundation for practice. PLC actions are guided by such educational values and beliefs. It is critical for school leaders to believe that all of their students deserve an equitable and quality education. When PLCs are not thoughtfully implemented and there is a drop in student achievement or growth over time, the district will need to be courageous and reassess its use of PLCs.

It could be argued that this study was targeted at principals and school leaders who already felt positive about their past and current PLC experiences. Regardless of their disposition toward PLCs, informants from this study noted that PLCs are difficult to 
implement; this dissertation identified this educational problem, and worked to address it. The Estacada PLC handbook, Guiding the Work of Professional Learning Communities: Perspectives for School Leaders is one answer to the challenge of implementing PLCs effectively. Through the R\&D process, a handbook has been developed that can successfully guide principals and school leaders through their PLC work, particularly in a small, rural school district. There is still a great deal to learn, explore, and discover about PLCs. However, what is certain is that there is an unequivocal need for their continuous improvement in today's school systems. A specific recommendation from the Estacada design team is to reconfigure its four schools into $\mathrm{K}-2,3-5,6-8$, and 9-12 levels and to bring like-minded PLCs closer together under one roof. Hopefully, this will make PLCs in Estacada more efficient and productive in addressing student needs and school change initiatives.

Giles and Hargreaves (2006) told the story of three schools using PLCs to make the point that schools often succumb to change forces that undermine PLC work, including: competitive pressure, evolutionary attrition of change, and standardized reform. My own experience with PLCs in Estacada yields a point that is different. What I take away from my own experience with PLCs in Estacada is that the district has become stronger in its collaborative work as opposed to reverting to bad habits. PLC members in Estacada have become less competitive and are more willing to share their ideas and resources. Regarding the evolutionary attrition of change, I have found that PLCs have become more robust, especially with the advent of the handbook. I will acknowledge that prescriptive and standardized reform through mandated and highly suggested school 
change initiatives is a source of frustration for PLC members in Estacada. However, using the handbook has allowed PLCs to address school change initiatives in a more productive and efficient manner. As a result, I conclude that the PLC handbook has brought the Estacada School District closer together, and has set the stage for the accomplishment of future goals and objectives.

Through conversations with educators from across the state and nation, it is evident that PLCs are not always sustainable or fully implemented. Many times the efforts for change are overcome by resistance, and the original reason for change is abandoned. The Estacada PLC handbook promotes the idea that though collaboration, shared leadership, data-driven decision-making, and fidelity to the PLC process, working in PLCs is not only doable, but it can positively impact a variety of factors that contribute to student learning and shape the school, district, and community culture. Though I concede that PLCs are not always sustainable or fully implemented, I still insist that teamwork rather than people working in isolation will lead to better decision making. Moreover, it is not only principals leading the way in Estacada. PLCs are also leading the way with contributions from all of their participants including principals, teachers, support staff, parents, families, and community members. PLCs appear to have a bright future in Estacada, but my biggest fear is that the benefits of PLCs will not be maximized if the reconfiguration of our schools is too disruptive. Estacada has the facility space, the necessary personnel, and the know-how to dramatically improve teaching and learning. However, moving students and staff from one school to another will undoubtedly be an adjustment, and the long-term benefits will need to outweigh the short-term discomforts. 
I sincerely hope that Estacada will reconfigure into a central campus and capitalize even more from the critical work of its PLCs.

The deeper success of PLCs in Estacada, and in other school districts, lies with the people who work and live in the community. Do they see a need for the improvement of all students? Are they taking into account school, district, and community challenges as a whole? Can the schools, district, and community create a shared vision that promotes social justice and utilizes a collaborative approach through PLCs? Principals and school leaders who are not afraid to make changes and challenge current policies, past practices, and norms for the betterment of all students have the ability to make breakthroughs in the achievement of students each and every year. The Estacada PLC handbook, Guiding the Work of Professional Communities: Perspectives for School Leaders, was developed to help school leaders make a positive difference in the years to come. 


\section{REFERENCES}

Adair, M. M. (2012). The common core initiative: Dimensions of implementation. Monroe Township, NJ: New Jersey Association for Supervision and Curriculum Development.

Adams, J. (2009, February 3-5). Risk management: The economics and morality of safety revisited. Safety-critical systems: Problems, process and practice. Proceedings of the $17^{\text {th }}$ Safety-Critical Systems Symposium, Bright, United Kingdom.

American Educational Research Association. (2005). Teaching teachers: Professional development to improve student achievement. Research Points: Essential Information for Education Policy, 3(1), 1-4.

Anderson, K., Harrison, T., \& Lewis, K. (2012). Plans to adopt and implement common core state standards in the southeast region states. Regional Education Laboratory, REL 2012(136), 1-34.

Andrews, D., \& Lewis, M. (2002). The experience of a professional community: Teachers developing a new image of themselves and their workplace. Educational Research, 44(3), 237-254.

Apgar, D. (2006). Risk intelligence: Learning to manage what we don't know. Boston, MA: Harvard Business School Publishing.

Bakula, N. (2010). The benefits of formative assessments for teaching and learning. Science Scope, 1, 1-8.

Balyer, A. (2012). Transformational leadership behaviors of school principals: A qualitative research based on teachers' perceptions. International Journal of Educational Sciences, 4(3), 581-591.

Berry, B., Johnson, D., \& Montgomery, D. (2005). The power of teacher leadership. Educational Leadership, 62(5), 56.

Bezzina, C. (2006). The road less traveled: Professional communities in secondary schools. Theory into Practice, 45(2), 159-167.

Black, P., \& William, D. (1998). Inside the black box: Raising standards through classroom assessment. Phi Delta Kappan, 80(12), 139-148. 
Bloom, B. S., Englehart, M. D., Furst, E. J., Hill, W. H., \& Krathwohl, D. R. (1956). Taxonomy of educational objectives: Cognitive domain, handbook I. New York, NY: David McKay Company.

Bogdan, R. C., \& Biklen, S. K. (2007). Qualitative research for education: An introduction to theories and methods. Boston, MA: Pearson.

Borg, W. R., \& Gall, M. D. (1989). Educational research. White Plains, NY: Longman.

Bottery, M. (2003). The leadership of learning communities in a culture of unhappiness. School Leadership and Management, 23(2), 193-213.

Bridges, E., \& Hallinger, P. (1995). Implementing problem-based learning in leadership development. Eugene, OR: ERIC Clearinghouse on Educational Management.

Bruner, J. (1996). The culture of education. Cambridge, MA: Harvard University Press.

Buffum, A., Mattos, M., \& Weber, C. (2009). Pyramid response to intervention: RTI, professional learning communities, and how to respond when kids don't learn. Bloomington, IN: Solution Tree.

Calik, T., Sezgin, F., Kavgaci, H., \& Kilinc, A. (2012). Examination of relationships between instructional leadership of school principals and self-efficacy of teachers and collective teacher efficacy. Educational Sciences: Theory and Practice, 12(4), 2498-2504.

Cameron, G., McIver, M., \& Goddard, R. (2008). A different kind of community. Changing Schools, 57, 6-7.

Castanheira, P., \& Costa, J. A. (2011). In search of transformational leadership: A metaanalysis focused on the Portuguese reality. Social and Behavioral Sciences, 15(2011), 2012-2015.

Chenoweth, T. G., \& Everhart, R. B. (2002). Navigating comprehensive school change: A guide for the perplexed. Larchmont, NY: Eye on Education.

Chesterfield, R. A., Chesterfield, K. B, Hayes-Latimer, K., \& Chavez, R. (1983). The influence of teachers and peers on second language acquisition in bilingual programs. TESOL Quarterly, 17, 401-419.

Chew, J., \& Chan, C. C. (2008). Human resource practices, organizational commitment and intention to stay. International Journal of Manpower, 29(6), 503-522. 
Clark, I. (2011). Formative assessment: Policy, perspectives, and practice. Florida Journal of Educational Administration \& Policy, 4(2), 1-23.

Clark, R. C., \& Mayer, R. E. (2011). E-learning and the science of instruction: Proven guidelines for consumers and designers of multimedia learning (3rd ed.). San Francisco, CA: Pfeiffer.

Cleary, S., \& Malleret, T. (2007). Global risk: Business success in turbulent times. New York, NY: Palgrave Macmillan.

Cook, S., \& Yanow, D. (1996). Culture and organizational learning. In M. Cohen \& L. Sproull (Eds.), Organizational learning (pp. 373-390). Thousand Oaks, CA: Sage.

Cochran-Smith, M., \& Lytle, S. (1999). Relationships of knowledge and practice: Teacher learning in communities. Review of Research in Education, 24(1), 249-305.

Council of Chief State School Officers \& National Governors Association Center for Best Practices. (2010). Common core state standards initiative: Preparing America's students for college and career. Washington, DC: Authors.

Creswell, J. W. (2005). Educational research: Planning, conducting, and evaluating quantitative and qualitative research. Upper Saddle River, NJ: Pearson.

Crone, D. A., \& Horner, R. H. (2003). Building positive behavior support systems in schools. New York, NY: Guilford Press.

Crow, G. M., Matthews, L. J., \& McCleary, L. E. (1996). Leadership: A relevant and realistic role for principals. Larchmont, NY: Eye on Education.

Danielson, C. (2006). Teacher leadership that strengthens professional practice. Alexandria, VA: Association for Supervision and Curriculum Development.

Danielson, L., Doolittle, J., \& Bradley, R. (2007). Professional development, capacity building, and research needs: Critical issues for response to intervention implementation. School Psychology Review, 36(4), 632-637.

Darling-Hammond, L., Bullmaster, M. L., \& Cobb, V. L. (1995). Rethinking teacher leadership through professional development schools. The Elementary School Journal, 96(1), 87-106.

Darling-Hammond, L., \& McLaughlin, M. W. (1995, April). Policies that support professional development in an era of reform. Phi Delta Kappan, 76(8), 597-604. 
Datnow, A., Park, V., \& Wohlsteter, P. (2007). Achieving with data: How highperforming school systems use data to improve instruction for elementary students. Los Angeles, CA: University of Southern California, Rossier School of Education, Center on Educational Governance.

Dooner, A., Mandzuk, D., \& Clifton, R. (2007). Stages of collaboration and the realities of professional learning communities. Teaching and Teacher Education, 24, 564-574.

DuFour, R., DuFour, R., Eaker, R., \& Karhanek, G. (2004). Whatever it takes: How professional learning communities respond when kids don't learn. Bloomington, IN: Solution Tree.

DuFour, R., DuFour, R., Eaker, R., \& Many, T. (2006). Learning by doing: A handbook for professional learning communities at work. Bloomington, IN: Solution Tree.

Dunklee, D. R., \& Shoop, R. J. (2006). The principal's quick reference guide to school law: Reducing liability, litigation and other potential legal tangles. Thousand Oaks, CA: Corwin Press.

Dunn, R. (2009). Research on the Dunn and Dunn learning-styles model. Differentiating instruction for at-risk students: What to do and how to do it. Landham, MD: Rowman \& Littlefield.

Dunn, R., \& DeBello, T. C. (1999). Improved test scores, attitudes, and behaviors in America's schools: Supervisors' success stories. Westport, CT: Bergin and Garvey.

Dunne, F., Nave, B., \& Lewis, A. (2000). Critical friends groups: Teachers helping teachers to improve student learning. Phi Delta Kappan International Research Bulletin, 28, 9-12.

Eaker, R., \& Gonzalez, D. (2007). Leading in professional learning communities. National Forum of Educational Administration and Supervision Journal, 24(1), 6-13.

Echevarria, J., Vogt, M., \& Short, D. J. (2010). Making content comprehensible for secondary English learners: The SIOP model. Boston, MA: Pearson.

Edmonds, R. R. (1986). Characteristics of effective schools. The school achievement of minority children. Hillsdale, NJ: Lawrence Erlbaum.

Egan, K. (1992). Imagination in teaching and learning: The middle school years. Chicago, IL: The University of Chicago Press. 
Egan, K. (1997). The educated mind: How cognitive tools shape our understanding. Chicago, IL: The University of Chicago Press.

Egodawatte, G., McDougall, D., \& Stoilescu, D. (2011). The effects of teacher collaboration in grade 9 applied mathematics. Educational Research Policy Practice, 10, 189-209.

Eisenhart, C. (2007). The process of differentiated instruction: Through the lens of a reading coach. Retrieved March 28, 2013, from http://www.ride.ri.gov /instruction/curriculum/rhodeisland/ppts/DifferentiatingInstruction.pdf

Elmore, R., City, E., Fiarman, S., \& Teitel, L. (2009). Instructional rounds in education: A network approach to improving teaching and learning. Cambridge, MA: Harvard Education Press.

Elmore, R., \& Wisenbaker, J. (2000). The crabapple experience: Insights from program evaluations. Phi Delta Kappan, 82(4), 280-28 3.

Englert, C. S., \& Tarrant, K. L. (1995). Creating collaborative cultures for educational change. Remedial and Special Education, 16(6), 325-336.

Evanson, S. (2013). Grading and performance rubrics. Retrieved August 20, 2013, from http://www.cmu.edu/teaching/designteach/teach/rubrics.html

Farkas, R. D. (2003). Effects of traditional versus learning-styles instructional methods on middle school students. Journal of Educational Research, 97(1), 42-51.

Finn, C., \& Petrilli, M. (2010). Now what? Imperatives and options for common core implementation and governance. Washington, DC: Thomas B. Fordham Foundation.

Fisher, D., \& Frey, N. (2007). Checking for understanding: Formative assessment techniques for your classroom. Alexandria, VA: Association for Supervision and Curriculum Development.

Fogarty, R. J., \& Pete, B. M. (2005). How to differentiate learning. Thousand Oaks, CA: Corwin.

Fullan, M. (2009). Motion leadership: The skinny on becoming change savvy. Toronto, CA: Corwin Press.

Fulton, K., Yoon, I., \& Lee, C. (2005). Induction into learning communities. Washington, DC: National Commission on Teaching and America's Future. 
Gall, M. D., Borg, W. R., \& Gall, J. P. (1996). Educational research: An introduction. White Plains, NY: Longman.

Gardner, H. (1983). Frames of mind: The theory of multiple intelligences. New York, NY: Basic Books.

Gardner, H. (1999). The disciplined mind: What all students should understand. New York, NY: Simon \& Schuster.

Garet, M. S., Porter, A. C., Desimone, L., Birman, B. F., \& Suk Yoon, K. (2001). What makes professional development effective? Results from a national sample of teachers. American Educational Research Journal, 38(4), 915-945.

Garland, V. E. (2010). Emerging technology trends and ethical practices for the school principal. Journal of Educational Technology Systems, 38(1), 39-50.

Gaskill, P. J., \& Hoy, A. W. (2013). Fifty years of theory into practice: Learning from the past, looking to the future. Theory Into Practice, 52(1), 2-4.

Geijsel, F. P., Sleegers, P. J., Stoel, R. D., \& Kruger, M. L. (2009). The effect of teacher psychological, organizational and leadership factors on teachers' professional learning. Elementary School Journal, 109(4), 406-427.

Genesee, F., Lindholm-Leary, K., Saunders, W., \& Christian, D. (2009). English language learners in US schools: An overview of research findings. Journal of Education for Students Placed at Risk, 10(4), 363-385.

Gewertz, C. (2013). Interpretations differ on common core's nonfiction rule. Education Week, 32(21). Retrieved from http://www.edweek.org/ew/articles/ 2013/01/30/19nonfiction_ep.h32.html

Giles, C., \& Hargreaves, A. (2006). The sustainability of innovative schools as learning organizations and professional learning communities during standardized reform. Educational Administration Quarterly, 42(124), 124-156.

Goddard, R. D., Hoy, W. K., \& Hoy, A. W. (2000). Collective teacher efficacy: Its meaning, measure, and impact on student achievement. American Educational Research Journal, 37(2), 479-507.

Goulet, L., Krentz, C., \& Christiansen, H. (2003). Collaboration in education: The phenomenon and process of working together. The Alberta Journal of Educational Research, 49(4), 325-340. 
Greenwood, C. R., Kratochwill, T., R., \& Clements, M. (2008). Schoolwide prevention models: Lessons learned in elementary schools. New York, NY: The Guildford Press.

Hackman, J. R. (1976). Group influences on individuals. Handbook of industrial organizational psychology. Chicago, IL: Rand McNally.

Halverson, R., Prichett, R. B., \& Watson, J. G. (2007). Formative feedback systems and the new instructional leadership. Madison, WI: University of WisconsinMadison, School of Education, Wisconsin Center for Education Research. Retrieved February 15, 2013, from http://www.wcer.wisc.edu/publications

Hargreaves, A. (2000). Four ages of professionalism and professional learning. Teachers and Teaching: Theory and Practice, 6(2), 151-182.

Hargreaves, A. (2001). The emotional geographies of teachers' relations with colleagues. International Journal of Educational Research, 35(5), 503-527.

Harmony Education Center. (2013). Critical friends consultancy protocol. Retrieved March 27, 2013, from http://www.nsrfharmony.org/protocol/doc/consultancy.pdf

Harris, A., \& Jones, M. (2010). Professional learning communities and systems improvement. Improving Schools, 13(2), 172-181.

Haslam, B. (1998). How to rebuild a local professional development infrastructure. In New American Schools, Getting better by design (vol. 4). Arlington, VA: New American Schools.

Hoerr, T. R. (2005). The art of school leadership. Alexandria, VA: Association for Supervision and Curriculum Development.

Hollins, E. R., McIntyre, L. R., DeBose, C., Hollins, K. S., \& Towner, A. (2004). Promoting a self-sustaining learning community: Investigating an internal model for teacher development. International Journal of Qualitative Studies in Education, 17(2), 247-264.

Hughes, T. A., \& Kritsonis, W. A. (2006). A national perspective: An exploration of professional learning communities and the impact on school improvement efforts. National Journal for Publishing and Mentoring Doctoral Student Research, 1(1), $1-12$.

Jackson, A. W., \& Davis, G. A. (2000). Turning points 2000: Educating adolescents in the $21^{\text {st }}$ century. New York, NY: Teachers College Press. 
Johnsen, S. (2003). Adapting instruction with heterogeneous groups. Gifted Child Today, 26(3), 5-6.

Johnson, S. M., \& Kardos, S. M. (2002). Keeping new teachers in mind. Educational Leadership, 59(6), 12-16.

Joyce, B., \& Showers, B. (1995). Student achievement through staff development: Fundamentals of school renewal. White Plains, NY: Longman.

Kaufman, M. T. (2003, February 24). Robert K. Merton, versatile sociologist and father of the focus group. The New York Times. Retrieved from http://www.nytimes. com/2003/02/24/ nyregion/robert-k-merton-versatile-sociologist-and-father-ofthe-focus-group-dies-at-92.html

Kindler, A. L. (2002). Survey of the states' limited English proficient students and available educational programs and services. Washington, DC: National Clearinghouse for English Language Acquisition.

Kohn, A. (2000). The case against standardized testing. Portsmouth, NH: Heinemann.

Kratochwill, T. R., Volpiansky, P., Clements, M., \& Ball, C. (2007). Professional development in implementing and sustaining multitier prevention models: Implications for response to intervention. School Psychology Review, 36, 618631.

Lambert., L., Walker, D., Zimmerman, D., Cooper, J., Lambert, M., Gardner, M., \& Slack, P. (1995). The constructivist leader. New York, NY: Teachers College Press.

Lambert, L., Walker, D., Zimmerman, D., Cooper, J., Lambert, M., Gardner, M., \& Szabo, M. (2002). The constructivist leader $\left(2^{\text {nd }}\right.$ ed.). New York, NY: Teachers College Press.

Leithwood, K. (2007). Transformation: Principalship in a transactional policy world. San Francisco: CA: John Wiley.

Leithwood, K., \& Jantzi, D. (2006). Transformational school leadership for large scale reform: Effects on students, teachers, and their classroom practices. School Effectiveness and School Improvement, 17(2), 201-228.

Leonard, L., \& Leonard, P. (2001). Assessing aspects of professional development in schools: Beliefs versus practices. The Alberta Journal of Educational Research, 47(1), 4-23. 
Lezotte, L. (1997). Learning for all. Okemos, MI: Effective Schools Products.

Little, J. W., Gearhart, M, Curry, M., \& Kafka, J. (2003). Looking at student work for teacher learning, teacher community, and school reform. Phi Delta Kappan, 85(3), 184-192.

Lorenz. R. A., \& Pichert, J. (1989). Evaluation of education program developments: Illustration of the research and development cycle. The Diabetes Educator, 15(3), 253-256.

Louis, K. S., \& Kruse, D. (1995). Professionalism and community: Perspectives on reforming urban schools. Thousand Oaks, CA: Corwin.

Louis, K. S., Marks, H., \& Kruse, S. (1996). Teachers' professional community in restructuring schools. American Educational Research Journal, 33(4), 757-798.

Lynch, S. A., \& Warner, L. (2008). Creating lesson plans for all learners. Kappa Delta Pi Record, 45(1), 10-15.

Marzano, R. J., Waters, T., \& McNulty, B. A. (2005). School leadership that works: From research to results. Alexandria, VA: Association for Supervision and Curriculum Development.

Matthews, L. J., \& Crow, G. M. (2010). The principalship: New roles in a professional learning community. Boston, MA: Allyn \& Bacon.

McAdamis, S. (2001). Teachers tailor their instruction to meet a variety of student needs. Journal of Staff Development, 22(2), 1-5.

McLaughlin, M., \& Talbert, J. (2001). Professional communities and the work of high school teaching. Chicago, IL: University of Chicago Press.

McNeil, M. (2009). 46 states agree to common academic standards effort. Education Week, 28(33), 16.

Merriam, S. B. (1998). Qualitative research and case study applications in education. San Francisco, CA: John Wiley \& Sons.

Meyer, C. A. (1992). What's the difference between authentic and performance assessment? Educational Leadership, 49, 39-40.

Mokhtari, K., Thoma, J., \& Edwards, P. (2009). How one elementary school uses data to help raise students' reading achievement. The Reading Teacher, 63(4), 334-337. 
Nanus, B. (1992). Visionary leadership: Creating a compelling sense of direction for your organization. San Francisco, CA: Jossey-Bass.

Natural Resources Digital Library. (2014). Rural communities explorer. Retrieved April 6, 2014, from www.oregonexplorer.info/rural

Northouse, P. G. (2001). Leadership theory and practice. Thousand Oaks, CA: Sage.

Ogawa, R. T., \& White, P. A. (1994). School-based management: An overview. In S.A. Mohrman \& P. Wohlstetter (Eds.), School-based management: Organizing for high performance (pp. 53-80). San Francisco: CA: Jossey-Bass.

Ogonsky, A. (2008). The response to intervention handbook: Moving from theory to practice. New York, NY: Park Place Publications.

Oregon Department of Education. (2013). State board of education adopts changes to graduation proficiency requirements. Retrieved March 2, 2013, from www.ode.state.or.us/new/announcements

Oregon Department of Education. (2014a). Educator effectiveness: Who is evaluated under SB 290? Retrieved April 6, 2014, from http://www.ode.state.or.us/wma/ teachlearn/educatoreffectiveness/guidance-for-sb-290-evaluations.pdf

Oregon Department of Education. (2014b). Next generation of accountability. Retrieved April 6, 2014, from http://www.ode.state.or.us/search/page/?id=3475

Owens, R. (1998). Organizational behavior in education (6 ${ }^{\text {th }}$ ed.). Needham Heights, MA: Allyn \& Bacon.

Pajak, E. (2000). Approaches to clinical supervision: Alternatives for improving instruction. Norwood, MA: Christopher-Gordon.

Perry, L. (2007). The impact of risk management on the changing nature of a principal's work. Queensland, Australia: Queensland University of Technology.

Peterson, K., \& Deal, T. (1999). Shaping school culture: The heart of leadership. San Francisco, CA: Jossey-Bass.

Phillips, J. (2003). Powerful learning: Creating learning communities in urban school reform. Journal of Curriculum and Supervision, 18(3), 240-258.

Pollock, J. E. (2007). Improving student learning one teacher at a time. Alexandria, VA: Association for Supervision and Curriculum Development. 
Popham, W. J. (2008). Transformative assessment. Alexandria, VA: Association for Supervision and Curriculum Development.

Posselt, J. R., Jaquette, O., Bielby, R., \& Bastedo, M. N. (2012). Access without equity: Longitudinal analyses of institutional stratification by race and ethnicity, 19722004. American Educational Research Journal, 49(6), 1074-1111.

Prasse, D. P. (2009). Why adopt an RTI model? Retrieved February 12, 2013, from the RTI Action Network at www.rtinetwork.org/Learn/Why/ar/WhyRTI

Prestine, N. A. (1993). Extending the essential schools metaphor: Principal as enabler. Journal of School Leader, 3(4), 356-379.

Reeves, D. B. (2006). The learning leader: How to focus school improvement for better results. Alexandria, VA: Association for Supervision and Curriculum Development.

Reutzel, D. R., Cooter, R. B., \& Blake, B. E. (2008). Teaching children to read: The teacher makes the difference. Upper Saddle River, NJ: Pearson.

Riehl, C. J. (2000). The principal's role in creating inclusive schools for diverse students: A review of normative, empirical, and critical literature on the practice of educational administration. Review of Educational Research, 70(1), 55-81.

Rosenholtz, S. J. (1985). Effective schools: Interpreting the evidence. American Journal of Education, 93(3), 352-388.

Sailor, W., Dunlap, G., Sugai, G., \& Horner, R. (2009). Handbook of positive behavior support. Eugene, OR: Springer.

Sawyer, R. K. (2006). The Cambridge handbook for the learning sciences. New York, NY: Cambridge University Press.

Schmoker, M. (2006). Results now. Alexandria, VA: Association for Supervision and Curriculum Development.

Schmoker, M. (2011). Focus: Elevating the essentials to radically improve student learning. Alexandria, VA: Association for Supervision and Curriculum Development.

Schmuck, R. A. (2006). Practical action research for change. Thousand Oaks, CA: Corwin. 
Scribner, J. P., Cockrell, K. S., Cockrell, D. H., \& Valentine, J. W. (1999). Creating professional communities in schools through organizational learning: An evaluation of a school improvement process. Educational Administration Quarterly, 35(1), 130-160.

Senge, P. M. (1990). The fifth discipline: The art and practice of the learning organization. New York, NY: Doubleday.

Senge, P. M. (1999). The dance of change: The challenges to sustaining momentum in learning organizations. New York, NY: Currency-Doubleday.

Senge, P. M., Cambron-McCabe, N., Lucas, T., Smith, B., Dutton, J., \& Kleiner, A. (2000). Schools that learn: A fifth discipline fieldbook for educators, parents, and everyone who cares about education. New York, NY: Doubleday.

Sergiovanni, T. J. (1995). The principalship: A reflective practice perspective. Boston, MA: Allyn and Bacon.

Shields, C. M. (2003). Good intentions are not enough: Transformative leadership for communities of difference. Lanham, MD: Scarecrow Press.

Shinn, M. R., Walker, H. M., \& Stoner, G. (2002). Interventions for academic and behavior problems II: Preventive and remedial approaches. Bethesda, MD: NASP Publications.

Sidhu, R. (2011, June). Why use AFT? Dusting off the black box. Teaching Geography, 36(2), 58-60.

Simonsen, B., Eber, L., Black, A. C., Sugai, G., Lewandowski, H., Sims, B., \& Myers, D. (2010). Illinois statewide positive behavioral interventions and supports: Evolution and impact on student outcomes across years. Journal of Positive Behavior Interventions, 4(1), 5-12.

Sisman, M. (2004). Instructional leadership ( $2^{\text {nd }}$ ed.). Ankara, Turkey: Pegem A. Publications.

SMARTER Balanced Assessment Consortium. (2010). An overview of the SMARTER Balanced Assessment Consortium. Retrieved February 1, 2013, from www.weraweb.org/pages/activities/WERA_Winter 10/2.2\%20SBAC_Overview.pdf.

Snow, C. E., Cancino, H., Gonzalez, P., \& Shriberg, E. (1987). Second language learners' formal definitions: An oral language correlate of school literacy. Los Angeles, CA: University of California, Center for Language Education and Research. 
Snow-Gerono, J. L. (2004). Professional development in a culture of inquiry: PDS teachers identify the benefits of professional learning communities. Teaching and Teacher Education, 21(3), 241-256.

Starr, K. (2012). Problematizing risk and the principalship: The risky business of managing risk in schools. Educational Management Administration and Leadership, 40(4), 464-479.

Stiggins, R. (2005). Assessment for learning. Portland, OR: Assessment Training Institute.

Stiggins, R., \& DuFour, R. (2009). Maximizing the power of formative assessments. Phi Delta Kappan, 90(9), 640-644.

Strahan, D. (2003). Promoting a collaborative professional culture in three elementary schools that have beaten the odds. The Elementary School Journal, 104(2), 127146.

Subban, P. (2006). Differentiated instruction: A research basis. International Education Journal, 7(7), 935-947.

Sugai, G., Horner, R. H., Algozzine, R., Barret, S., Lewis, T., Anderson, C., \& Simonsen, B. (2010). School-wide positive behavior support: Implementers' blueprint and self-assessment. Eugene, OR: University of Oregon.

Sugai, G., Lewis, T., Todd, A., \& Horner, R. H. (2001). School-wide evaluation tool. Eugene, OR: University of Oregon.

Supovitz, J. A. (2002). Developing communities of instructional practice. Teachers College Record, 104(8), 1591-1626.

Supovitz, J. A., \& Christman, J. B. (2003). Developing communities of instructional practice: Lessons for Cincinnati and Philadelphia (CPRE Policy Brief RB-39). Philadelphia, PA: University of Pennsylvania, Consortium for Policy Research in Education.

Thompson, D. C., Gregg, L., \& Niska, J. M. (2004). Professional learning communities, leadership, and student learning. Research in Middle Level Education Online, 28(1). Retrieved September 10, 2012, from http://www.nmsa.org/Publications/ RMLEOnline/tabid/101/Default.aspx

Tomlinson, C. A. (2001). How to differentiate instruction in mixed-ability classrooms. Alexandria, VA: Association for Supervision and Curriculum Development. 
Tomlinson, C. A., \& McTighe, J. (2006). Integrating differentiated instruction and understanding by design. Alexandria, VA: Association for Supervision and Curriculum Development.

Tschannen-Moran, M., \& Hoy, A. W. (2001). The differential antecedents of selfefficacy beliefs of novice and experienced teachers. Teaching and Teacher Education, 23(6), 944-956.

Ubben, G. C., Hughes, L. W., \& Norris, C. J. (2004). The principal: Creative leadership for excellence in schools. Boston, MA: Pearson.

University of California Berkeley. (2013). 2013 Social justice symposium. Retrieved March 27, 2013, from http://www.socialwelfare.berkeley.edu/sjs/

University of South Alabama. (2013). Qualitative data analysis. Retrieved March 28, 2013, from http://www.southalabama.edu/coe/bset/johnson/lectures/lec17.pdf

Vanderford, L. (2012). Revised Bloom's taxonomy (handout). Clackamas, OR: Clackamas Educational Service District.

Van Lare, M. D., \& Brazer, S. D. (2013). Analyzing learning in professional learning communities: A conceptual framework. Leadership and Policies in School, 12(4), 374-396.

VanTassel-Baska, J., Feng, A. F., Brown, E., Bracken, B., Stambaugh, T., French, H., McGowan, S., Worley, B., Quek, C., \& Bai, W. (2008). A study of differentiated instructional change over three years. Gifted Child Quarterly, 52(4), 297-312.

Vescio, V., Ross, D., \& Adams, A. (2006). A review of research on professional learning communities: What do we know? Gainesville, FL: University of Florida.

Vescio, V., Ross, D., \& Adams, A. (2008). A review of research on the impact of professional learning communities on teaching practice and student learning. Teaching and Teacher Education, 24, 80-91.

Vygotsky, L. S. (1978). Mind in society. Cambridge, MA: Harvard University Press.

Wayman, J. C., Cho, V., Jimerson, J. B., \& Spikes, D. D. (2012). District-wide effects on data use in the classroom. Education Policy Analysis Archives, 20(25), 1-28.

Weick, K. E. (1979). The social psychology of organizing. Reading, MA: AddisonWesley. 
Wenger, E. (1998). Communities of practice: Learning, meaning, and identity. New York, NY: Cambridge University Press.

Wenglingsky, H. (2000). How teaching matters. Princeton, NJ: Educational Testing Service.

Whitaker, T. (2003). What great principals do differently: Fifteen things that matter most. Larchmont, NY: Eye on Education.

Wilson, S., \& Berne, J. (1999). Teacher learning and the acquisition of professional knowledge: An examination of research on contemporary professional development. Review of Research in Education, 24, 173-209.

Wood, R., \& Bandura, A. (1989). Social cognitive theory of organizational management. Academy of Management Review, 14(3), 361-384.

Wormeli, R. (2006). Fair isn't always equal: Assessing and grading in the differentiated classroom. Portland, ME: Stenhouse Publishers.

Zepeda, S. J. (2004). Leadership to build learning communities. The Educational Forum, 68(2), 144-151. 
APPENDIX

ESTACADA PLC HANDBOOK 


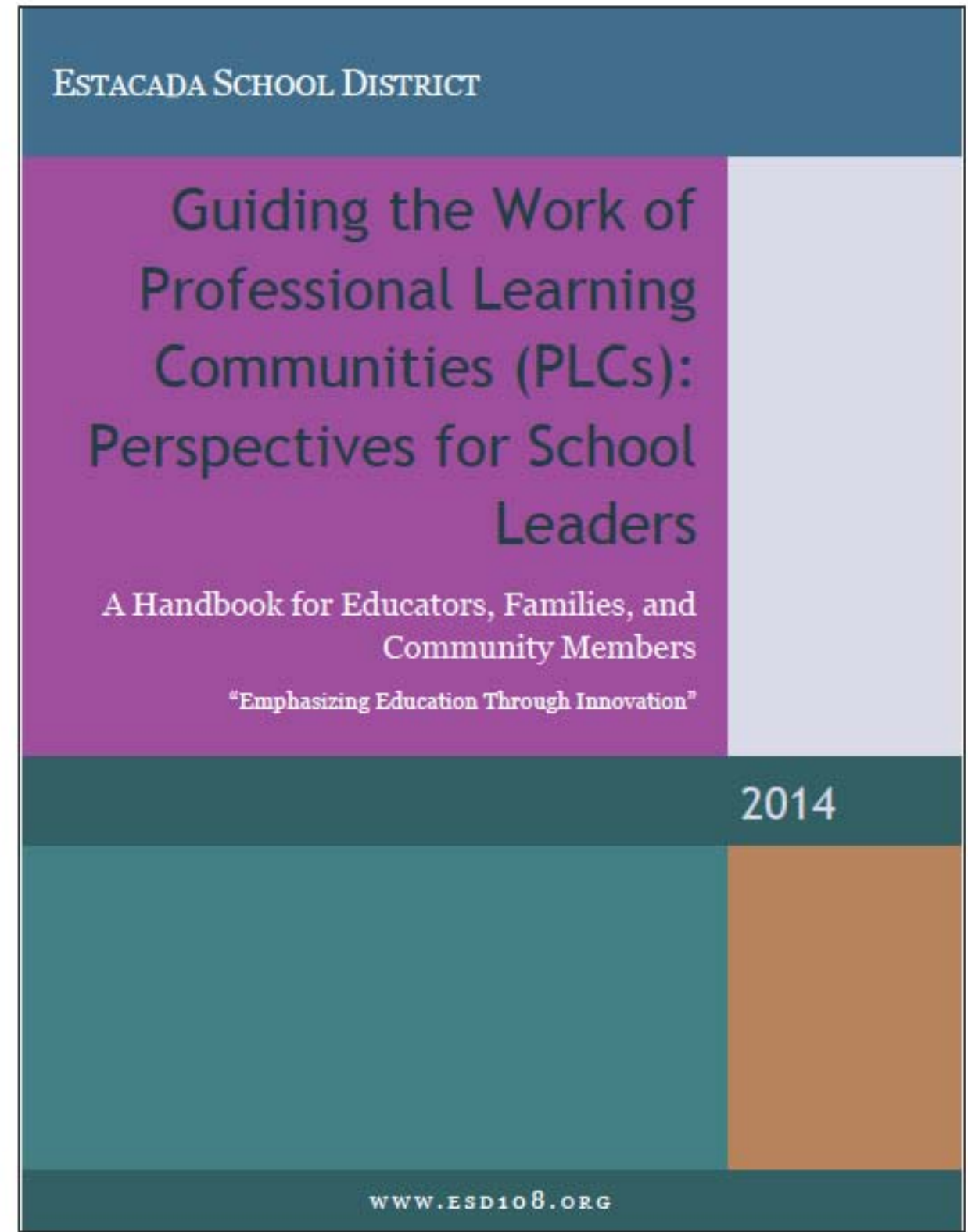




\section{Guiding the Work of Professional learning Gommunities:}

A Handbook for School Leaders

C 2014 Estacada School District - Estacada, OR

All rights reserved. No part of this handbook may be reproduced or transmitted in any form or by any means without written permission from the authors. 
"Quality teaching requires strong professional learning communities. Collegial interchange, not isolation, must become the norm for teachers. Communities of learning can no longer be considered utopian; they must become the building blocks that establish a new foundation for America's schools."

(National Commission on Teaching, 2003, p. 17)

"Districts need to identify and integrate resources and services from the community to strengthen school programs, family practices, and student learning and development."

(Joyce Epstein - Partnership Center for the Social Organization of School, 2013, p. 6) 


\section{Who Should Read This Handbook?}

The audience for this handbook is the administrators, teachers, support staff, families, and community members of the Estacada School District. As a district community, we face adversity and work to create a shared vision so that we can help one another do what is best for our students. With the Great Recession, we have often been left to wonder what resources will be available from year to year. Our community is to be commended for its perseverance. Truly, the greatest resource we have as a community is each other. We must lean on each other, and continue to do what is best for students, regardless of the obstacles we may face. In addition, we must find new, innovative ways to connect educators to families and community members through the PLC process so that we can all be a part of the shared vision, and contribute to moving the district forward.

"Education, then, beyond all other devices of human origin, is the great equalizer of the conditions of men, the balance-wheel of the social machinery."

-Horace Mann 


\section{How Should This Handbook Be Used?}

The activities in this handbook allow educators, families, and community members to participate in the PLC process in the Estacada School District. Administrators presented concepts included in this handbook to staff during the August 2013 inservice week. The activities were designed to provide a "back to basics" approach to PLCs in Estacada. The handbook attempts to clarify key terms used in Estacada's continuous improvement plan. It also aims to define the prevalent school change initiatives that are currently being addressed by the district. The handbook emphasizes that working in PLCs is what allows the district to complete the important work associated with major initiatives. The activities in this handbook should be repeated throughout the course of a school year, and should be part of the district's ongoing professional development.

Families and community members can use this handbook to better understand the district's priorities. They can also use the handbook to understand how they can become involved in helping to shape the district's future. Families and community members can partner with educators to help create the shared the vision that we want to provide for our students. 


\section{Table of Contents}

Who Should Read This Handbook? $-4$

How Should this Handbook be used? 5

With Appreciation. 9

Purpose of the Handbook n

What are PLCs, and what do they do? 12

The Four Key Questions of PlCs. $-13$

Getting Started: Establishing Team Norms. 14

Activity 1.0 - "We Will Statements"

Critical lssues for PLC Consideration

Activity 2.0 - "PLC Survey: What is our Current Reality?" 17

SMART Goals.

Activity 3.0 - "SMART Goal Worksheet" $-19$

PLC Minutes, Tasks, and Reflections

Activity 4.0 - "PLC Weekly Meeting Minutes Form".

Activity 5.0 - "PLC Tasks".

Activity 6.0 - "Reflection of PLC Work".

How can PLCs Support Differentiated/Sheltered Instruction?

Activity 7.0 - "Revised Bloom's Taxonomy Question Starters"

Peer Coaching - An Extension of PLC Work

Activity 8.0 - "Differentiated/Sheltered Instruction Walk-Through Tool. 34

How can PLCs Support RTI \& PBIS? 35

Activity 9.0 - "Pyramid of Interventions" 37

Activity 10.0 - "Monthly Academic Check". $-42$

Activity 11.0 - "Monthly Behavior Check" $-43$

How can PLCs Support Cormmon Core State Standards? $-44$ 
Activity 12.0 - "Tdentifying Priority Standards"

How can PLCs Support Proficiency-Based Learning?

Activity 13.0 - "Analyzing Your Common Assessment" ___ 47

Activity 14.0 - "Creating Student-Friendly Rubrics"____ 49

Activity 15.0 - "Incomplete Contract Agreement" _.______._. 53

District/Community Collaboration___

How can PLCs Support Grant Writing?

Activity 16.0 - Grant Writing: Getting Started___ 56

How can PLCs Support Consensus Building?.

Activity 17.0 - Building Consensus___ 58

How can PLCs Support After School Enrichment Programs?_____ 60

Activity 18.0 - After School Enrichment Programs___ $\quad 60$

How can PLCs Support P-3 Alignment?

Activity 19.0 - Planning for P-3 Alignment__ 61

How can PLCs Support Instructional Technology?

Activity 20.0 - Technology Mini Grant Application___ 66

How can PLCs Support Community Service Projects? _________ 68

Activity 21.0 - Memorandum of Understanding for a Fiscal Partner Relationship._._____...69

How can PLCs Support Communication Between the District \& Community?

Activity 22.0 - Communication between the District \& Community

Final Thoughts

$-75$ 


\section{List of Figures}

1.0 - The PLC Cycle (pg. 11)

2.0 - Differentiated Classroom Instructional Framework (pg. 29)

3.0 - Sheltered Classroom Instructional Framework (pg. 30)

4.0 - Essential Components of RT1 (pg. 36)

5.0 - PBIS Continuum of School-Wide Support (pg-36)

6.0 - Common Interests of the Estacada School District \& Community of Estacada (pg- 55)

\section{list of Tables}

1.0 - Prevalent School Change Initiatives (pg. 26)

2.0 - The Guiding Principles behind the Prevalent School Change Initiatives (pg. 27) 


\section{With Appreciation}

Activity 1.0, p. 15: Adapted from DuFour, R., DuFour, R., Eaker, R., \& Many, T. (2006). Learning by doing. Bloomington, $\mathbb{I N}$ : Solution Tree.

Activity 2.0. p. 17: Adapted from DuFour, R, DuFour, R, Eaker, R. \& Many, T. (2006). Learning by doing. Bloomington, $\mathbb{N}$ : Solution Tree.

Activity 3.0, p. 19: Adapted from Matthews, ㄴ.f. \& Crow. (2010). The principalship: New roles in a professional learning community. Boston, MA: Allyn \& Bacon.

Activity 4.0, p. 21: Adapted from Marzano, R.. Waters, T., and McNulty, B.A. (2005). School leadership that works: From research to results. Alexandria, VA: ASCD.

Activity 5.0, p. 22: Adapted from DuFour, R., DuFour, R, Eaker, R., \& Many. T. (2006). Learning by doing. Bloomington, $1 \mathrm{~N}$ : Solution Tree.

Activity 6.0, p. 24: Adapted from Mattthews, L. \& Crow, G.M. (2010). The principalship: New roles in a professional learning community. Boston, MA: Allyn \& Bacon.

Activity 7.0. p. 3: Adapted from Bloom, B.S., Englehart, M.D., Furst, E.J., Hill, W.H., \& Krathwohl, D.R. (1956). Taxonomy of educational objectives: Cognitive domain, handbook $L$. New York: David McKay Co.

Activity 8.0, p. 34: Adapted from Echevarria, J. Vogt, M., and Short, D. (2010). Making content comprehensible for secondary English learners: The SIOP model, Boston, MA: Pearson.

Activity 9.0, p. 37: Adapted from Buffum, A., Mattos, M., \& Weber, C. (2009). Pyramid response to intervention: $R T 7$, professional learning communities, and how to respond when kids don't learn. Bloomington, $\mathbb{1}$ : Solution Tree.

Activity 11.0, p. 43: Adapted from Sailor, W., Dunlap, G., Sugai, G., and Horner, R. (2009). Handbook of positive behavior support. Eugene, OR-Springer.

Activity 12.0. p. 45: Adapted from Schmoker, M. (2006). Results Now. Alexandria, VA: ASCD.

Activity 13.0, p. 47: Adapted from Eaker, R. \& Keating. J. (2011). Every school, every team, every classroom. Bloomington, $\mathbb{1 N}$ : Solution Tree

Activity 14.0, p. 49: Adapted from Stiggins, R. (2005). Assessment for learning. Portland, ORAssessment Training Institute.

Activity 16.0, p. 57: Adapted from Ford Leadership Institute. (2013). Rural Development Initiative Roseburg, OR: Ford Family Foundation. 
Activity 17.0, p. 58: Adapted from Ford Leadership Institute. (2013). Rural Development Initiative. Roseburg, OR: Ford Family Foundation.

Activity 21.0, p. 69: Adapted from Ford Leadership Institute. (2013). Rural Development Initiative. Roseburg, OR: Ford Family Foundation. 


\section{The Purpose of this Handbook}

To provide the stakeholders of the Estacada School District with common language and practical tools to use in the following areas:

OThe PLC Framework

OContent \& Curriculum

OCollecting \& Analyzing Data

OSupporting School Change Initiatives

OPlanning for Future Action

OTroubleshooting Challenges

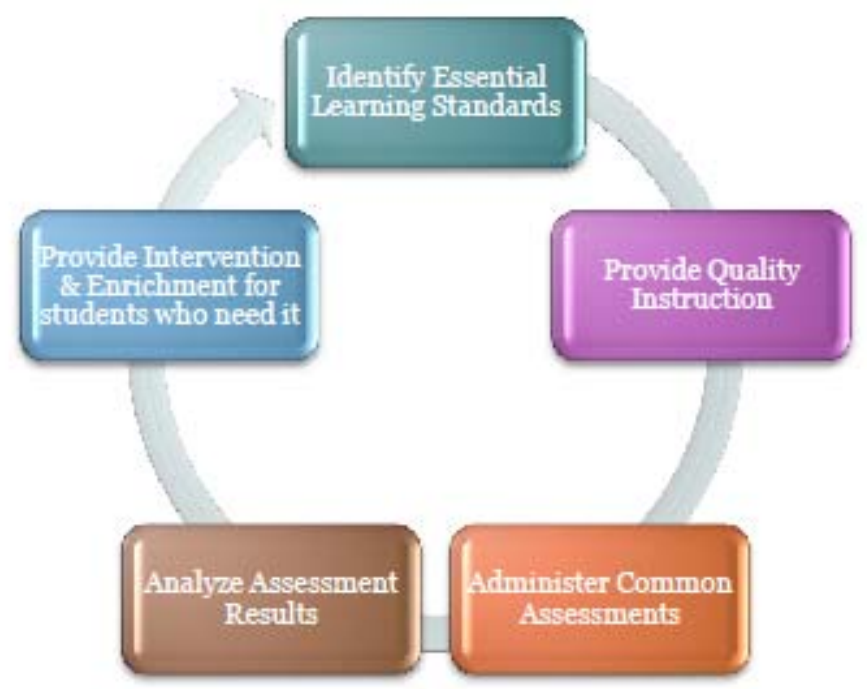

Eigure 1.0

The PLC Cycle (adapted from DuFour, DuFour, Eaker, and Many, p. 121) 


\section{What are Professional Learning Communities (PLCs), and what do they do?}

\section{Professional Learning Communities (PLCs)}

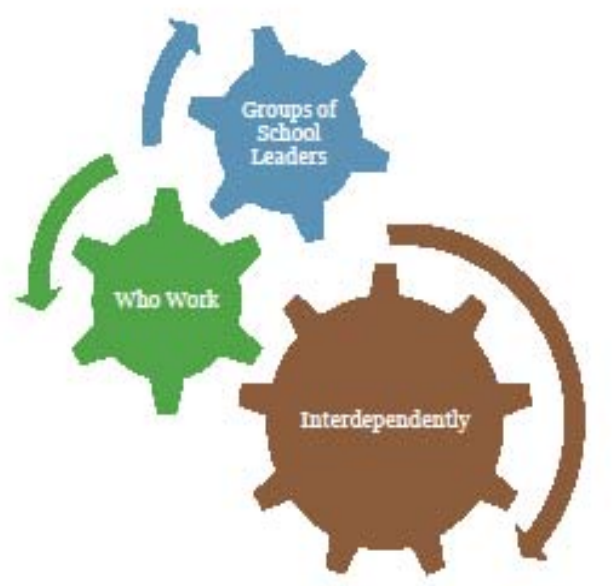

Examples of PLCs within a School:

*Three $3{ }^{\text {rd }}$ Grade Teachers

${ }^{*}$ Four Middle School Science Teachers

${ }^{*}$ Five High School Social Studies Teachers

What are PLCs supposed to do?

1. Identify the standards they will address.

2. Create and administer common assessments that address those standards.

3. Analyze the results of common assessments.

4. Plan intervention and enrichment opportunities for students based on the assessment results. 


\section{The Four Key Questions for PLCs}

1. What do we want our students to learn? Answer: Priority Standards (taken from the Common Core State Standards)

2. How will we know they have learned it? Answer: Data from Common Assessments

3. What will we do for students who have not yet demonstrated proficiency on standards? Answer: Intervention

4. What will we do for students who have already demonstrated proficiency and are ready to move on?

Answer: Enrichment 


\section{Getting Started: Establishing Team Norms}

It is important for PLCs to establish norms for their meetings. Our school district has implemented a one hour early release for students once a week so that PLCs can meet and conduct their business. Establishing Team Norms helps to ensure that PLCs operate in a respectful and productive manner.

\section{Establishing Team Norms}

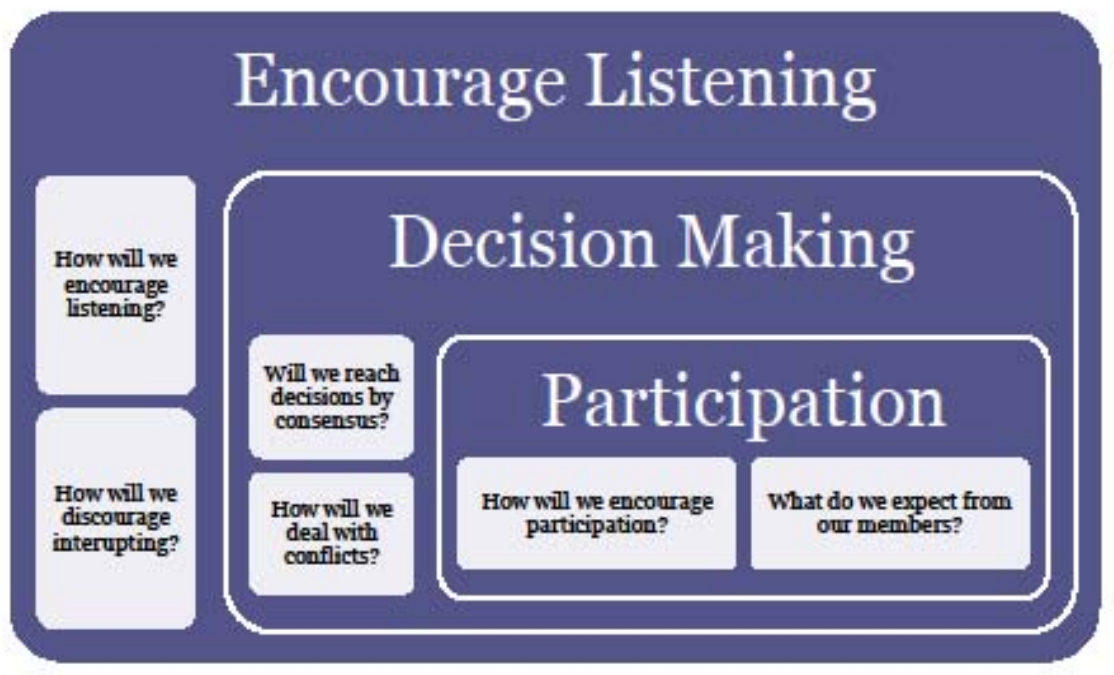




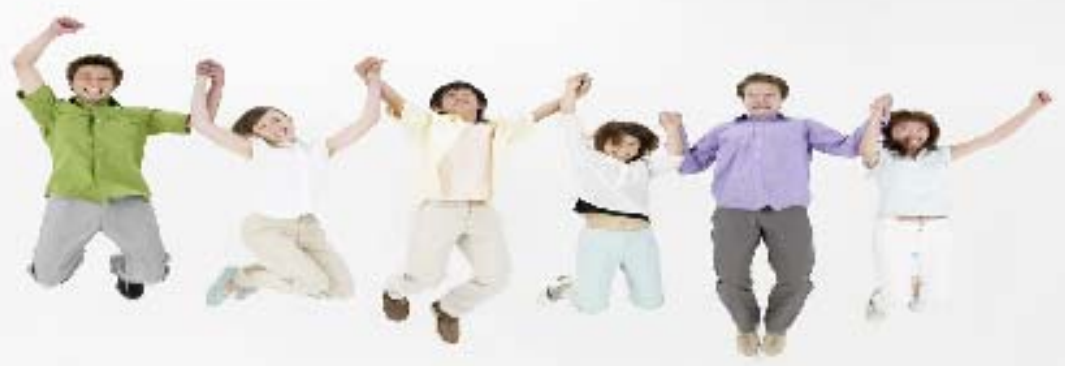

\section{ACTIVITY,}

PLCs should consider the graphic organizer "Establishing Team Norms" on the previous page. Then, PLCs should develop 5-7 "We will" statements. Each PLC should share their "We will" statements with their principal. PLCs should print these, and put them in the front of the PLC binder that they will bring to each meeting. Below are some examples of "We will" statements from the National Staff Development Council (2006).

- We will work together as a community that values consensus rather than majority rule.

- We will be fully present at the meeting by becoming familiar with materials before we arrive and by being attentive to behaviors which affect physical and mental engagement.

- We will invite and welcome the contributions of every member and listen to each other.

- We will be involved to our individual level of comfort. Each of us is responsible for airing disagreements during the meeting rather than carrying those disagreements outside the meeting.

- We will operate in a collegial and friendly atmosphere.

- We will keep confidential our discussions, comments, and deliberations. 


\section{Critical Issues for PLC Consideration}

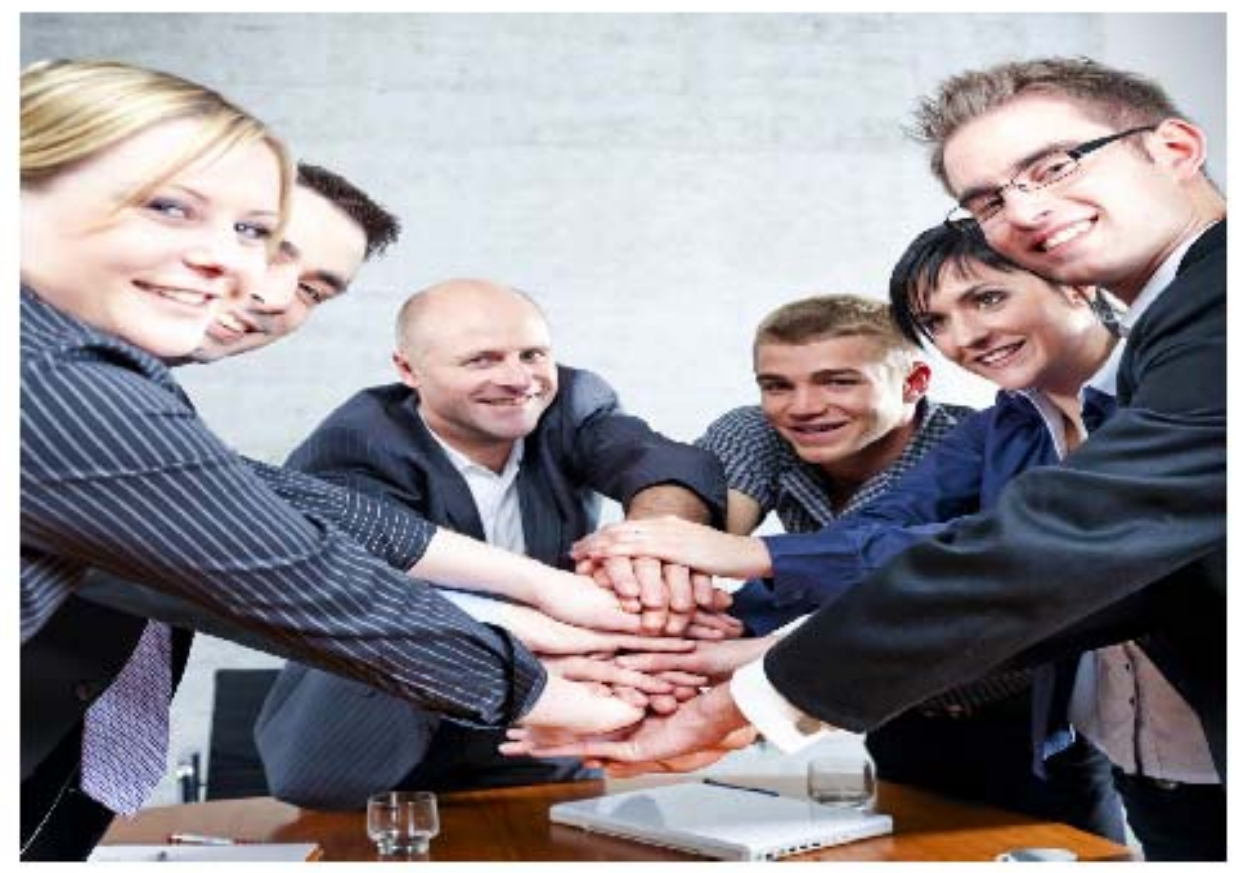

At the beginning of each specified unit of time (beginning of the year, semester, or unit), PLCs must get a sense of their current reality.

\section{$\operatorname{ACTIVITY2,0}_{2}$}

PLCs should complete the "PLC Survey: What is our Current Reality?" on the next page. Team members could fill out the survey individually first, then compare and discuss their answers with fellow PLC members. This will give PLCs an idea of where they are, and where they want to go. 


\section{PLC Survey: What is our Current Reality?}

Mark 1 for "Not True of Our Team"

Mark 2 for "Our Team is Addressing This"

Mark 3 for "True of Our Team"

1 - We have identified team norms and protocols to guide us in working together.

2. We have established SMART goals to help us understand what we would like to achieve with our students (SMART goals = Strategic, Measurable, Attainable, ResultsOriented, and Time-bound).

3. We have aligned our priority standards (from the Common Core State Standards) with the test specifications of our state assessment.

4 - We have agreed on how to best sequence the content of our course(s) and have established pacing guides to help students master the intended priority standards.

5. _-We have identified the prerequisite knowledge and skills students need in order to master the priority standards.

6. We have indentified strategies and created instruments to assess whether students have the prerequisite knowledge and skills.

7. _- We have developed interventions to assist students in acquiring prerequisite knowledge and skills when they lack in those areas.

8. We have developed frequent common formative assessments that help us to determine each student's mastery of priority standards.

9. We have established the level of proficiency we want to see from students for the priority standards.

10. - We use the results of our common assessments to assist each other in building on strengths and addressing weaknesses as part of an ongoing process of continuous improvement designed to help students achieve at higher levels.

II. We use the results of our common assessments to plan intervention and enrichment activities for students.

12. We have taught students the criteria we will use in judging the quality of their work and provided them with examples.

13. We have developed or utilized common summative assessments that help us assess the strengths and weaknesses of our program(s).

14. __ We use our PLC time to address Differentiated/Sheltered Instruction, PBIS/RTI, Common Core State Standards, and Proficiency-Based Learning.

15. W We formally evaluate our adherence to our team norms and the effectiveness of our team at least twice a year. 


\section{Writing SMART Goals as a PLC}

$S_{\text {trategic }}$

$M_{\text {easurable }}$

$\mathrm{A}_{\text {tainable }}$

$\mathrm{R}_{\text {ealls onioned }}$

$\prod_{\text {ime-Bound }}$

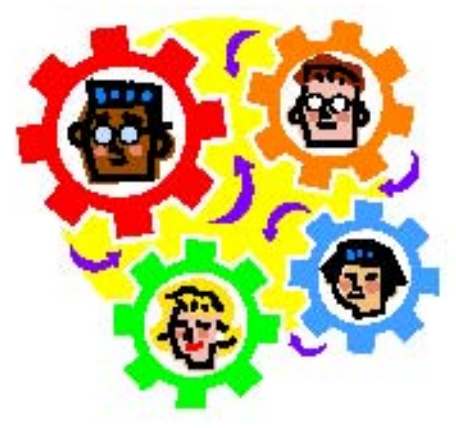

\section{$\operatorname{ACTIVITY}_{3.0}$}

PLCs should complete the SMART Goal Worksheet on the next page to identify what their SMART Goals are. SMART goals should be for a specified amount of time (i.e. - a year, semester, or unit of instruction). 


\section{SMART Goal Worksheet}

Strategic, Measurable, Attainable, Results-Oriented, Time-Bound

PLC Members:

SMART Goal:

How will we get there?

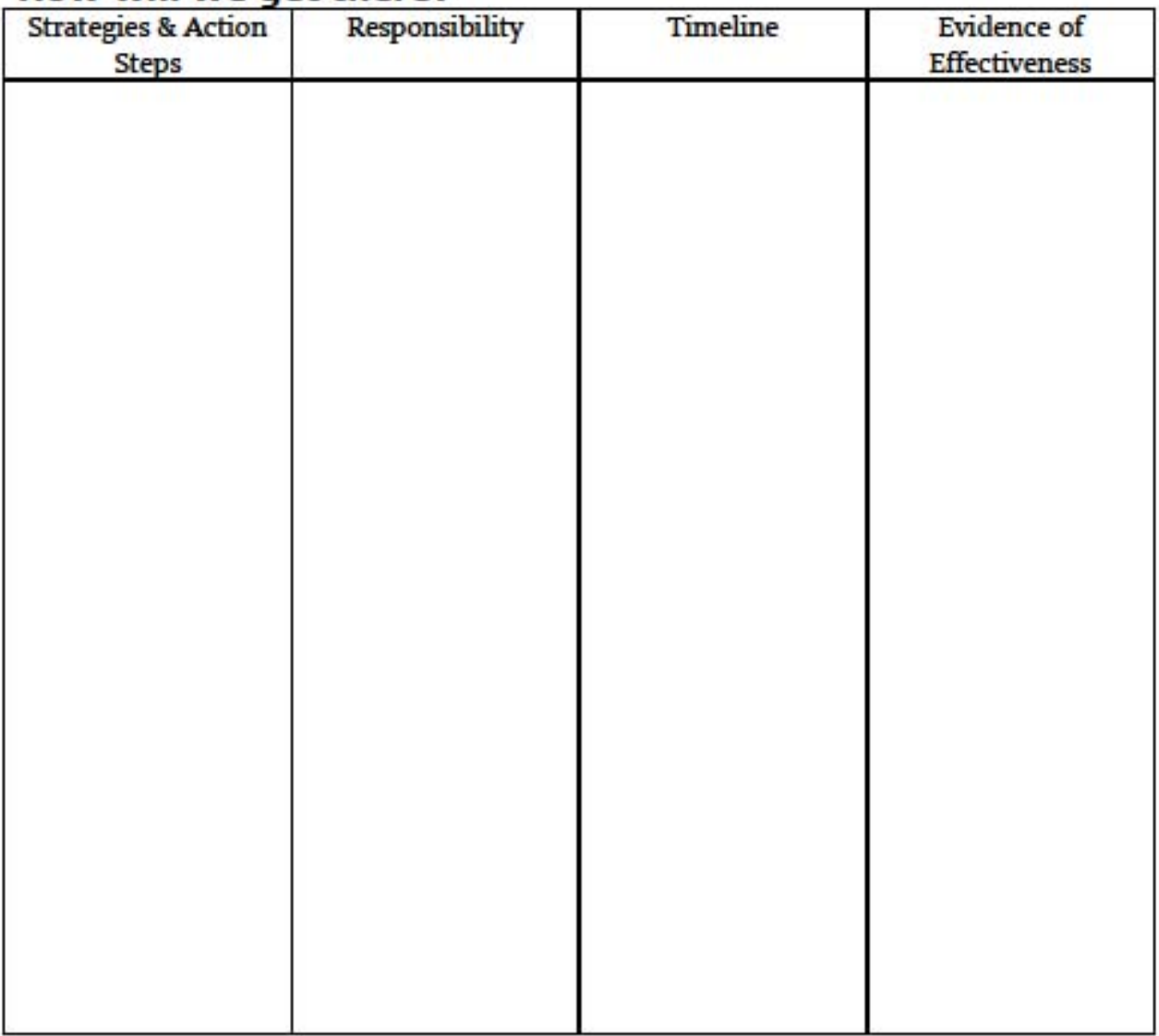




\section{PLC Minutes, Tasks, \& Reflections}

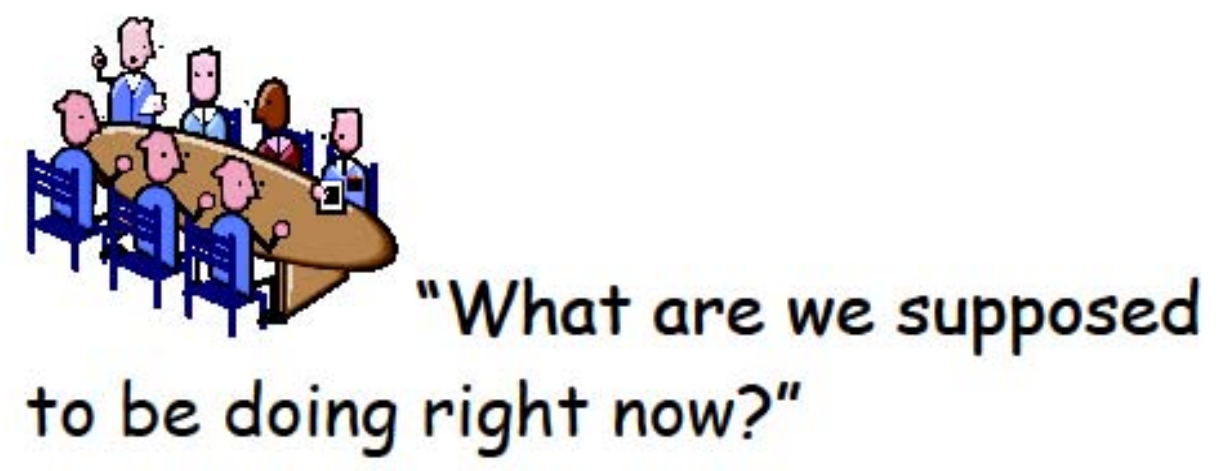

Ensure that this quote is never uttered at PLC meetings in your building. PLCs need guidance that will help them facilitate their meetings.

\section{$\operatorname{ACTIVIT}_{4} 0$}

PLCs should complete the PLC Weekly Meeting Minutes Form on the next page at each weekly PLC meeting. These should be turned in to the principal - preferably as soon as the meeting is over. Principals should have a mailbox, or designated area, for PLCs to put their minutes. The minutes form is helpful for PLC members as they run their meetings. PLCs should keep their minutes in a PLC binder so that they can refer back to previous meetings as they conduct their business. These minutes will also be helpful for administrators, both in terms of holding teachers accountable, and for keeping in touch with what each PLC is working on. 


\section{PLC Weekly Meeting Minutes Form}

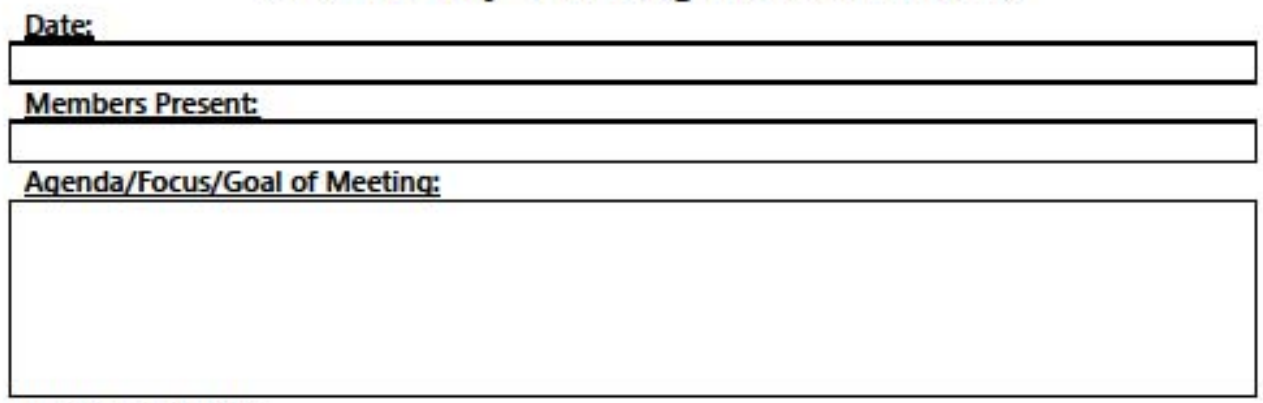

Discussion Points:

Decisions/Next Steps:

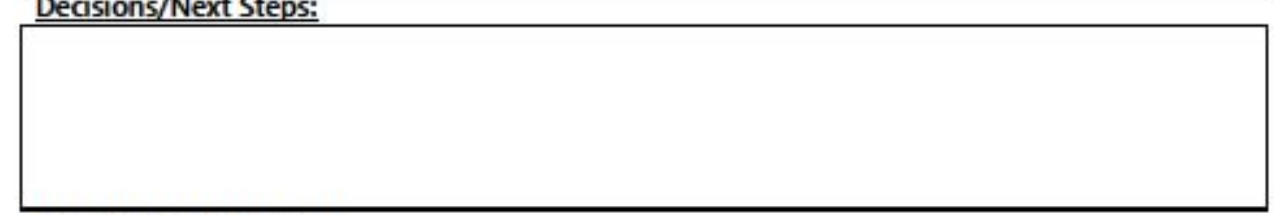

Items for Next Agenda:

\begin{tabular}{|c|c|c|}
\hline Items/Assionments for Next Meeting; & By When? \\
\hline What? & & \\
\hline & & \\
\hline & & \\
\hline & & \\
\hline
\end{tabular}

Questions/Concerns:

Is Administrative Support Request?

Yes Explain:

No

Location \& Date of Next Meetino: 


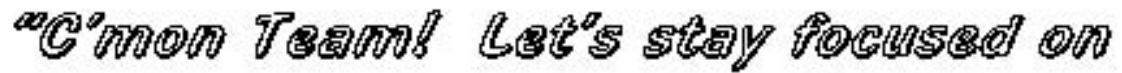

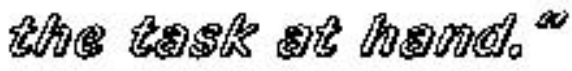

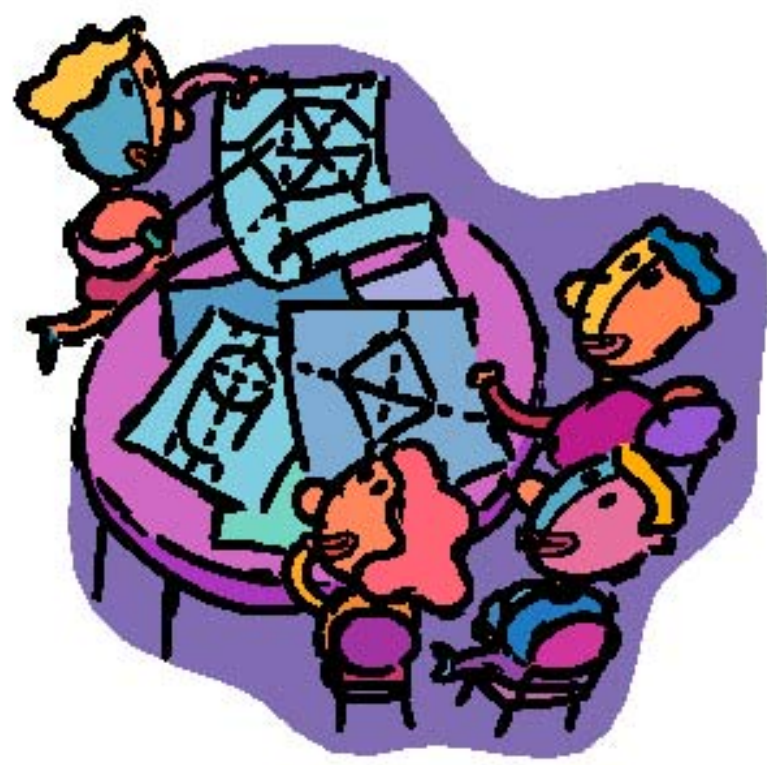

\section{$\mathrm{ACTIVITY}_{5.0}$}

It is important that PLCs understand the tasks they are supposed to be completing. The BLC Tasks form provided on the next page should be distributed at a staff meeting, and the principal should allow time for clarifying questions. Staff may write in the notes/clarifications section. The due dates provided are suggestions, and can vary depending on the needs of the school. 


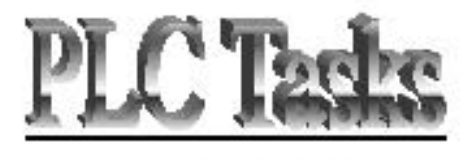

\begin{tabular}{|c|c|c|}
\hline Tasks & Notes/Clarification & Due Date \\
\hline $\begin{array}{l}\text { Complete PLC meeting } \\
\text { minutes }\end{array}$ & & Due each week \\
\hline Develop Team Norms & & Due by September $15^{\text {th }}$ \\
\hline $\begin{array}{l}\text { ldentify/clarify essential } \\
\text { learning standards (priority } \\
\text { standards) for your units of } \\
\text { study }\end{array}$ & & Due by October $1^{\text {st }}$ \\
\hline $\begin{array}{l}\text { Administer common } \\
\text { formative assessments }\end{array}$ & & At least one per month \\
\hline $\begin{array}{l}\text { Analyze results of common } \\
\text { assessments }\end{array}$ & & At least once per month \\
\hline $\begin{array}{l}\text { Develop a systematic plan } \\
\text { for intervention and } \\
\text { enrichment }\end{array}$ & & At least once per month \\
\hline $\begin{array}{l}\text { Develop a plan to celebrate } \\
\text { student improvement }\end{array}$ & & At least once per month \\
\hline $\begin{array}{l}\text { Develop a plan to celebrate } \\
\text { improvement of adults }\end{array}$ & & At least once per month \\
\hline
\end{tabular}


"There is an art of which every man should be a master of - the art of reflection. If you are not a thinking man, to what purpose are you a man at all?"

\section{-William Hart Coleridge}

\section{ACTIVITY 6.o}

Principals should encourage his/her PLCs to take time for reflection. These times of reflection should take place at natural breaks, such as the end of a unit, the end of a semester, or the end of the school year. PLCs should complete the Reflection of PLC Work form on the next page at a natural break that makes sense for the PLCs in your school.

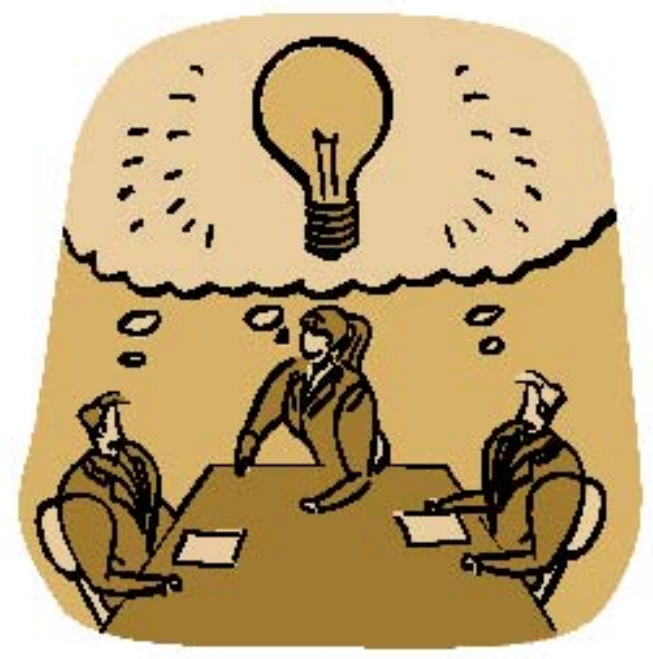




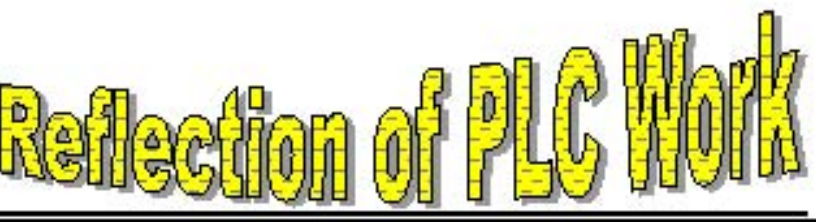

\section{What was our SMART Goal? \\ $\%$ of students will be proficient on}

Data for SMART Goal - Based on our common assessment(s), what is our current reality?

Assessment Results Analysis

- How many students passed?

- What affected the rate of success?

- What will we do differently next time?

Interventions (for those who struggled)

- How/when will we re-teach/re-assess?

- How will we provide more time/support?

Enrichment (for those who are proficient)

- How/when will we provide enrichment?

- How will we provide time/support?

What are the strengths of our PLC?

If we could change anything, we would...

Staff development that would help our PLC: 
Below you will see the main school change initiatives that PLCs in the Estacada School District are addressing.

Table 1.0 - Prevalent School Change Initiatives

\begin{tabular}{|c|c|c|c|c|c|c|}
\hline $\begin{array}{l}\text { School } \\
\text { Change } \\
\text { Initiatives }\end{array}$ & $\begin{array}{l}\text { Differentiated } \\
\text { Instruction } \\
\text { (DI) }\end{array}$ & $\begin{array}{l}\text { Sheltered } \\
\text { Instruction } \\
\text { (SI) }\end{array}$ & $\begin{array}{l}\text { Response to } \\
\text { Intervention } \\
\text { (RTI) }\end{array}$ & $\begin{array}{c}\text { Positive } \\
\text { Behavioral } \\
\text { Intervention } \\
\text { Support } \\
\text { (PBIS) }\end{array}$ & $\begin{array}{c}\text { Common } \\
\text { Core State } \\
\text { Standards } \\
\text { (CCSS) }\end{array}$ & $\begin{array}{l}\text { Proficiency- } \\
\text { Based } \\
\text { Learning }\end{array}$ \\
\hline Founders & $\begin{array}{l}\text { Tomlinson \& } \\
\text { McTighe (2006) }\end{array}$ & $\begin{array}{l}\text { Echevarria, } \\
\text { Vogt, \& } \\
\text { Short (2010) }\end{array}$ & $\begin{array}{l}\text { Buffum, } \\
\text { Mattos, \& } \\
\text { Weber (2009), } \\
\text { Organsky } \\
\text { (2008) }\end{array}$ & $\begin{array}{l}\text { Sailor, } \\
\text { Dumlap, } \\
\text { Sugai, \& } \\
\text { Horner (2009) }\end{array}$ & $\begin{array}{l}\text { Coumcil of } \\
\text { Chief State } \\
\text { School } \\
\text { Officers and } \\
\text { National } \\
\text { Governors } \\
\text { Association } \\
(2010)\end{array}$ & $\begin{array}{l}\text { Stiggins } \\
(2005), \\
\text { Wormelli } \\
(2006), \text { \& } \\
\text { Bakula } \\
(2010)\end{array}$ \\
\hline Key Goal & $\begin{array}{l}\text { To enable } \\
\text { learners at } \\
\text { mulriple levels } \\
\text { and abilities to } \\
\text { benefit from } \\
\text { instruction }\end{array}$ & $\begin{array}{l}\text { To explicitly } \\
\text { teach } \\
\text { functional } \\
\text { language } \\
\text { skills such as } \\
\text { how to } \\
\text { negotiate } \\
\text { meaning, } \\
\text { confirm } \\
\text { information, } \\
\text { argue, } \\
\text { persuade, } \\
\text { and disagree }\end{array}$ & $\begin{array}{l}\text { To introduce a } \\
\text { problem- } \\
\text { solving } \\
\text { process, } \\
\text { whose } \\
\text { foundation is } \\
\text { the provision } \\
\text { of systematic, } \\
\text { research- } \\
\text { based } \\
\text { instruction } \\
\text { and } \\
\text { interventions } \\
\text { for struggling } \\
\text { leamers }\end{array}$ & $\begin{array}{l}\text { To provide a } \\
\text { framework for } \\
\text { assisting } \\
\text { school } \\
\text { personnel in } \\
\text { adopting and } \\
\text { organizing } \\
\text { evidence- } \\
\text { based } \\
\text { behavioral } \\
\text { interventions } \\
\text { into an } \\
\text { integrated } \\
\text { continumm } \\
\text { that enhances } \\
\text { behavioral } \\
\text { outcomes for } \\
\text { all students }\end{array}$ & $\begin{array}{l}\text { To establish } \\
\text { a common } \\
\text { set of } \\
\text { expectations } \\
\text { across states } \\
\text { for what K- } \\
12 \text { students } \\
\text { are } \\
\text { expected to } \\
\text { know and } \\
\text { be able to } \\
\text { do }\end{array}$ & $\begin{array}{l}\text { To } \\
\text { comnmmicate } \\
\text { learning } \\
\text { targets up } \\
\text { front to } \\
\text { students, } \\
\text { ensure that } \\
\text { students } \\
\text { receive } \\
\text { continmous } \\
\text { feedback } \\
\text { from their } \\
\text { teachers, and } \\
\text { have nulliple } \\
\text { opportunities } \\
\text { to refine } \\
\text { student } \\
\text { learning }\end{array}$ \\
\hline
\end{tabular}


Table 2.0 - The Guiding Principles behind the Prevalent School Change Initiatives

\begin{tabular}{|c|c|}
\hline Differentiated Instruction (DI) & $\begin{array}{l}\text { - Learning opportunities allow for } \\
\text { student choice } \\
\text { - Learning opportunities are matched } \\
\text { with students' individual learning } \\
\text { profiles } \\
\text { - Cooperative learning is encouraged }\end{array}$ \\
\hline Sheltered Instruction (SI) & $\begin{array}{l}\text { - Learning opportunities involve the use } \\
\text { of visuals and graphic organizers } \\
\text { - Learning opportunities allow for } \\
\text { student-to-student interaction } \\
\text { - Important vocabulary is frontloaded } \\
\text { and students interact in a print-rich } \\
\text { learning environment }\end{array}$ \\
\hline Response to Intervention (RTI) & $\begin{array}{l}\text { - Universal screening assessments are } \\
\text { used with students school-wide } \\
\text { - Teachers analyze data in order to plan } \\
\text { for future intervention and enrichment } \\
\text { activities } \\
\text { - Teachers monitor student progress on } \\
\text { a regular and consistent basis }\end{array}$ \\
\hline $\begin{array}{l}\text { Positive Behavioral Intervention Support } \\
\text { (PBIS) }\end{array}$ & $\begin{array}{l}\text { - Learning activities are designed to be } \\
\text { preventative, not reactive } \\
\text { - School-wide learning activities } \\
\text { promote desired outcomes for all } \\
\text { - An emphasis on celebrating positive } \\
\text { results achieved by students, staff, and } \\
\text { the school as a whole }\end{array}$ \\
\hline Common Core State Standards (CCSS) & $\begin{array}{l}\text { - Schools place an emphasis on college } \\
\text { and career readiness } \\
\text { - Teachers emphasize reading, writing, } \\
\text { speaking, and listening activities with } \\
\text { students } \\
\text { - Literacy and language activities are } \\
\text { promoted across the curriculum } \\
\text { - Teachers focus on priority standards } \\
\text { that are weighted more heavily on state } \\
\text { assessments }\end{array}$ \\
\hline Proficiency-Based Learning & $\begin{array}{l}\text { - Learning targets and expectations are } \\
\text { communicated to students at the } \\
\text { beginning of units and lessons } \\
\text { - Multiple opportunities exist for } \\
\text { students to demonstrate improvement } \\
\text { and proficiency } \\
\text { - Teachers provide relevant and ongoing } \\
\text { feedback }\end{array}$ \\
\hline
\end{tabular}




\section{How can PLCs Support Differentiated \& Sheltered Instruction?}

Differentiated Instruction (DI) involves teachers providing specific ways for each individuals to learn as deeply as possible and as quickly as possible without assuming that one student's roadmap for learning is identical to anyone else's. Teachers leading differentiated classrooms use flexible groupings, cooperative learning, and varied questioning strategies. They also provide choices (or a menu of options) to meet the unique needs of each learner. Differentiated Instruction focuses on the premise of "I do, we do it, ya'll do it, you do it." PLC members attempt to address common learning targets with their students, but may not necessarily use the same instructional strategies. Hence, teachers are able to share their successes (and failures) in a risk-free environment and learn from one another.

Sheltered Instruction (SI) teaching strategies such as Guided Language Acquisition Design (GLAD) and Sheltered Instruction Observation Protocol (SIOP) are helpful for English Language Learners (ELLs), but for all students as well. It focuses on frontloading vocabulary in a print-rich environment by using visuals, graphic organizers, and sentence frames. It also emphasizes cooperative learning and student to student interaction. Principals can use the items in this section with their PLCs to promote sheltered instruction. Walk-through tools are also included, which could be used by the principal and/or peer coaches when observing a lesson. Peer coaching, in this context, is meant to be an extension of PLCs.

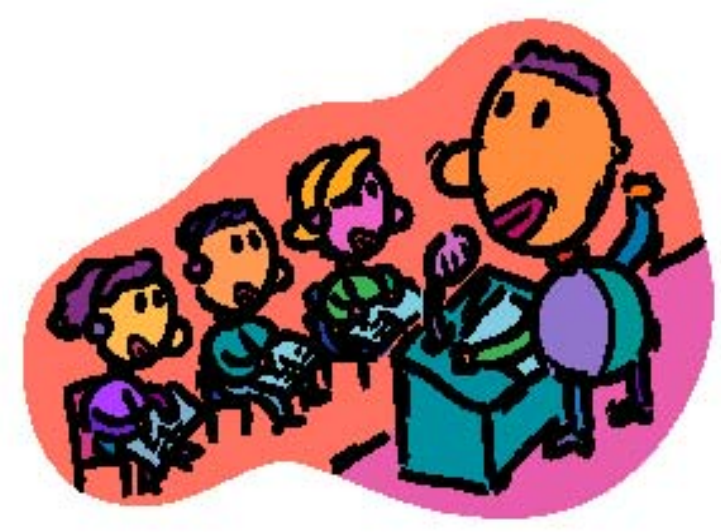




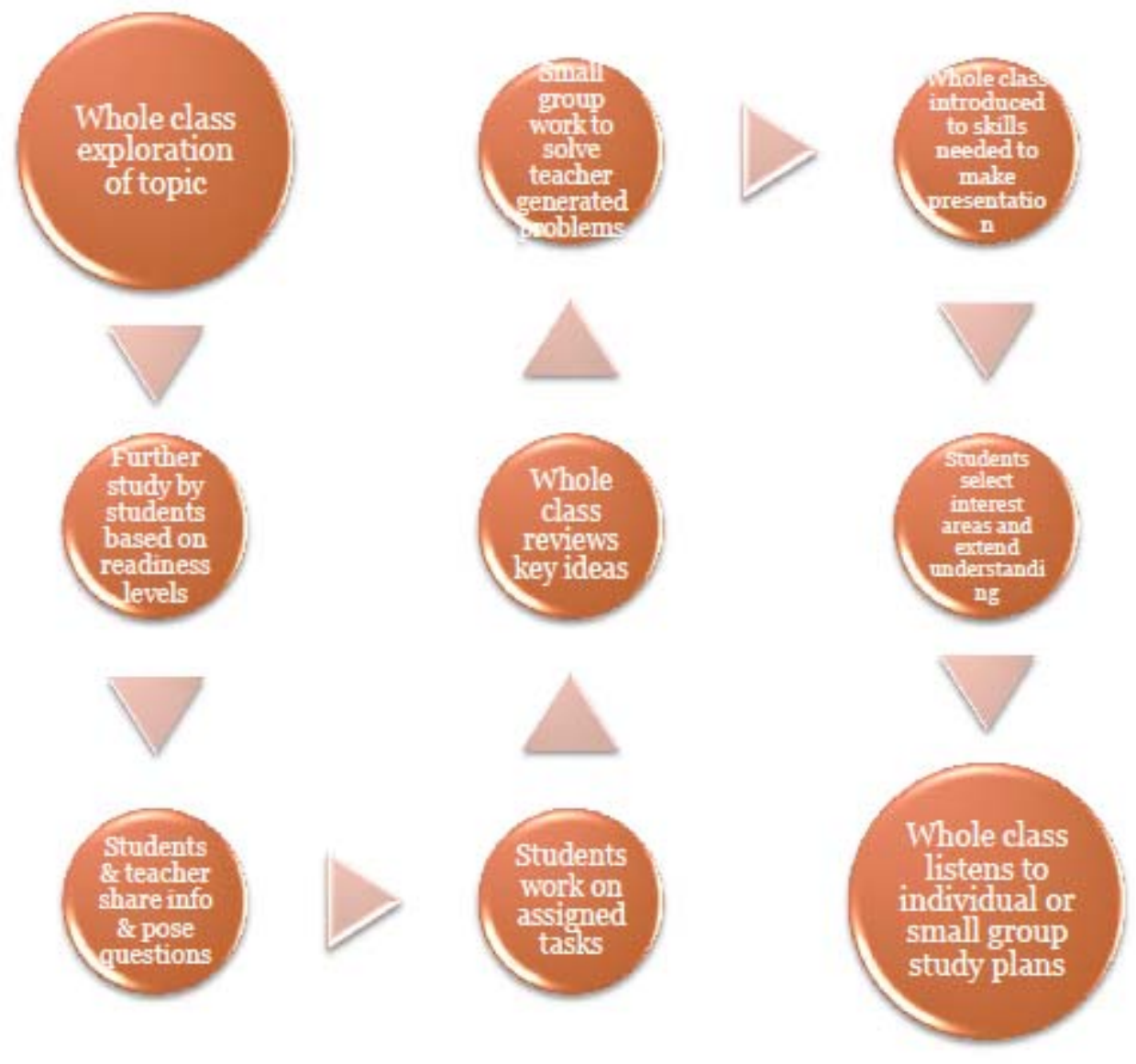

\section{Figure 20}

Differentiated Classroom Instructional Framework (Tomlinson, 20o1, p. 6) 


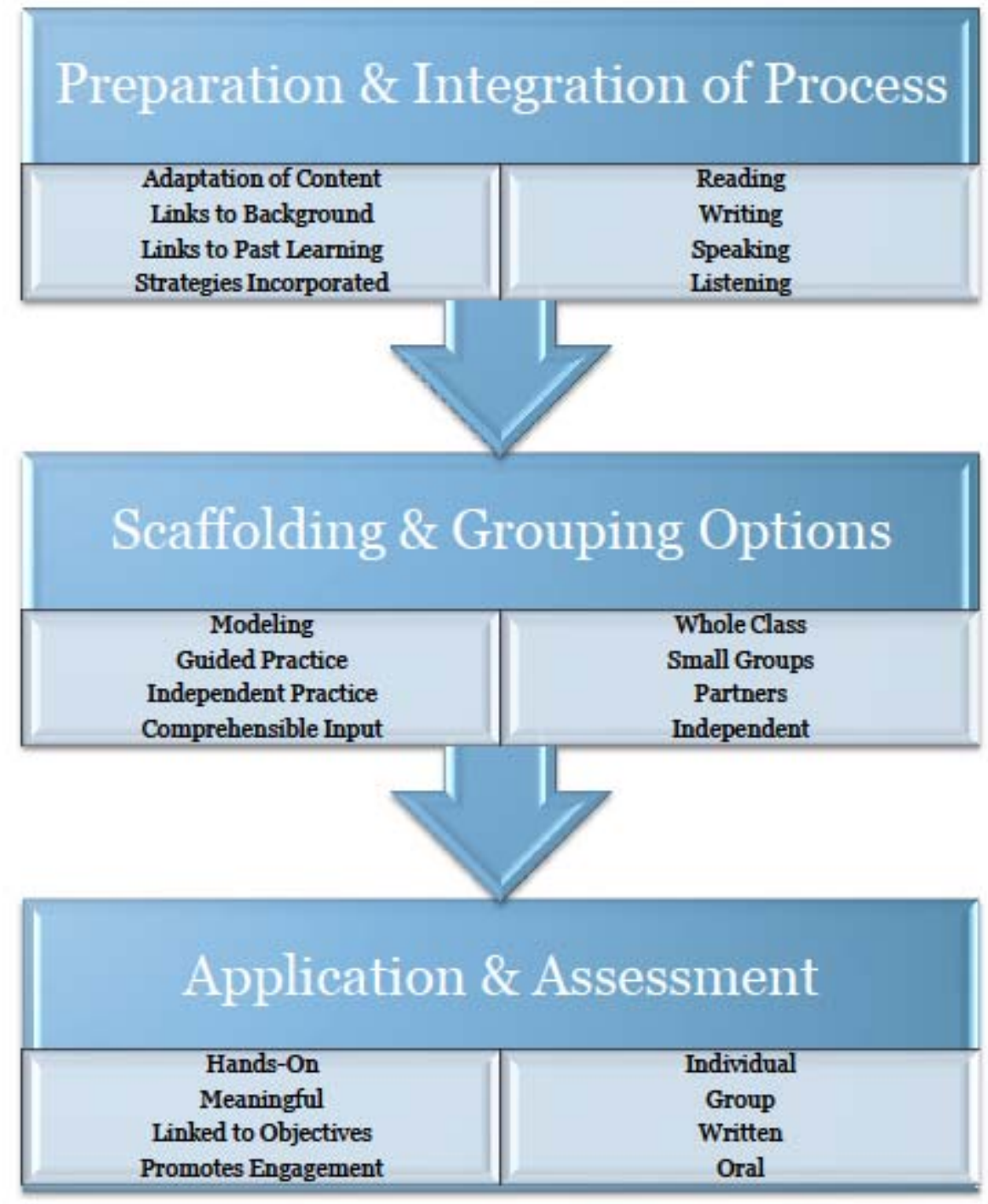

Figure 30

Sheltered Classroom Instructional Framework (Echevarria, Vogt \& Short, 2010, p. 50) 


\section{ACTIVITY $7.0^{-0}$}

As PLCs plan units of instruction, they should consider the Bloom's Taxonomy Ouestion Starters on the next page. A teacher emphasizing differentiated instruction provides a variety of questions that address Bloom's various domains. Teachers should consider each student's individual readiness level. Questions should build in terms of difficulty. The beginning domains will provide scaffolding, and the higher level domains will challenge the students to think more critically. Various formats for responding to questions should also be considered (choral response, individual response, written response, graphic organizers, projects, demonstrations, etc.). PLCs should then incorporate a variety of questioning strategies into their lesson plans.

"We cannot seek or attain health, wealth, learning, justice or kindness in general. Action is always specific, concrete, individualized, and unique."

\section{-John Dewey}

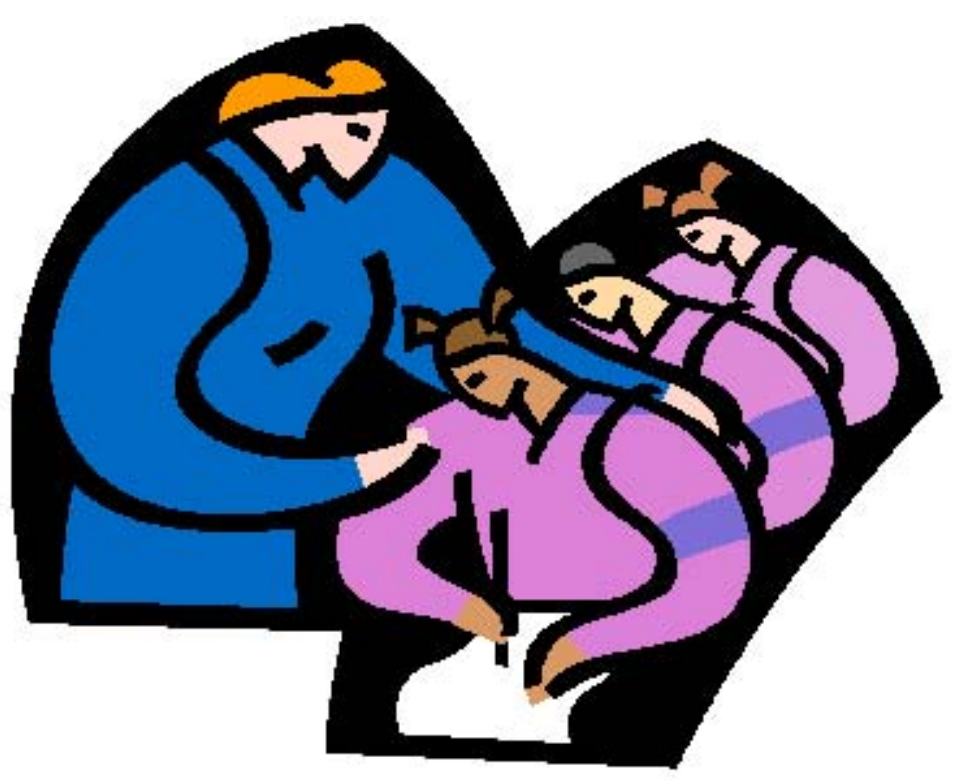




\section{Revised Bloom's Taxonomy Question Starters}

\section{Remembering}

- What did the book/article say about...?

- Define...?

- List the three...

- Explain the...

Understanding

- What can you conclude...?

- State in your own words...?

-What does this picture mean...?

- What reasons or evidence...?

Applying

- If you know $A$ and $B$, how could you determine $C$ ?

- What other possible reasons...?

- What might they do with...?

- What would do you suppose would happen if...?

Analyzing

- Compare/Contrast using a diagram.

- Distinguish between and

- List three ways is different from/similar to

- How is related to ?

\section{Evaluating}

- For what reasons would you favor...?

- Choose why made the decision to...

- Which will benefit good for the greatest number?

- Grade your efforts on...

\section{Creating}

- Can you develop a new way to...?

- Create a from the list below.

- What would you do if...?

- Invent a new using the existing parts. 


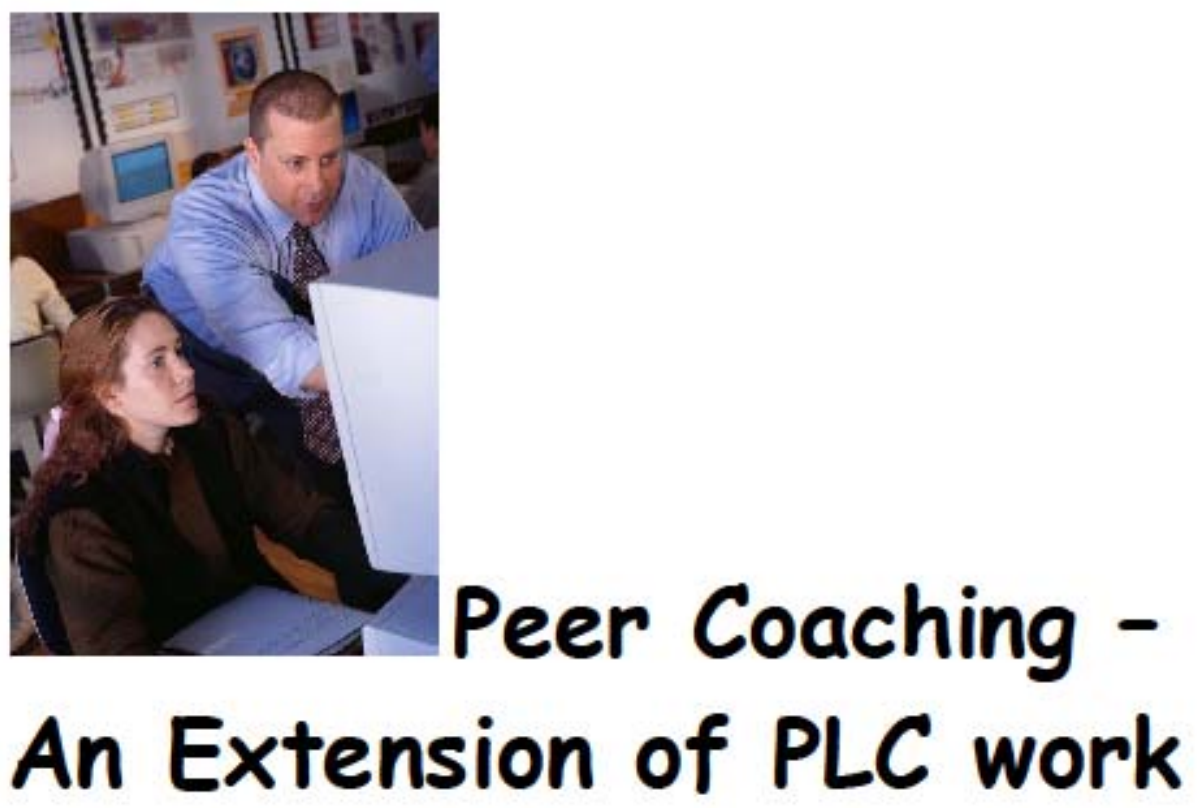

\section{ACTIVITY 8.0}

Having your PLCs meet during a weekly, uninterrupted meeting is a great start! However, your PLCs' discussions will be so much richer if you are able to allow for peer coaching as an extension to what PLCs are talking about in their meetings - particularly when it comes to Differentiated \& Sheltered Instruction. Teachers benefit from watching their peers. They also benefit from authentic, non-threatening feedback from their peers. There are several different approaches that can be taken:

- Have a few main teacher leaders serve as coaches for the building

- Allow all (or most) teachers the opportunity to observe peers and provide feedback

- Allow all (or most) teachers the opportunity to see resident experts doing model lessons

- Allow opportunities for team teaching

You will need to determine what works best for your building. Administrators will need to determine how to provide sub time (or coverage) so that this peer coaching element of PLCs can take place. Once opportunities for teachers to observe other teachers have been established, a tool they could use is the Differentiated/Sheltered Instruction WalkThrough Tool on the next page. This will help teachers provide feedback to their peers regarding their Differentiated \& Sheltered Instruction techniques. 
Differentiated/Sheltered Instruction Walk-Through Tool

Teacher: Grade Level/Subject:

Activity. Date:

Print Rich Environment 123 N/A Content \& language objectives clearly defined, displayed, and reviewed with students

Evidence of vocabulary tied to current unit(s) of instruction

Word walls (tied to current unit(s) of instruction)

Teacher-made charts/visuals/graphic organizers

Other:

Strategies/Interaction using Academic Language Scaffolding techniques consistently used, assisting and supporting student understanding

Grouping configurations support language and content objectives (interaction in pairs or small groups)

Specific teacher prompts expecting the use of current academic language/vocabulary

A variety of questions or tasks that promote higher-order thinking skills

Other:

Literacy Strategies across Curricular Areas

Evidence of current vocabulary instruction and use

Reading

Writing

Speaking

Listening

$\begin{array}{lll}1 & 2 & 3 \\ \text { N/A }\end{array}$

\begin{tabular}{|l|l|l|l|l|}
\multicolumn{1}{c|}{1} & \multicolumn{3}{c|}{3 N/A } \\
& & & & \\
\hline & & & & \\
\hline & & & & \\
\hline & & & & \\
\hline & & & & \\
\hline
\end{tabular}




\section{How can PLCs support Response to Intervention (RTI) \& Positive Behavioral Intervention Support (PBIS)?}

Response to Intervention (RTI) asserts that students who are not responsive to instructional interventions that are effective with most students are in need of timely, frequent, and intensive intervention to accelerate their progress and thereby avoid delays in attaining short-term benchmark and annual, grade level proficiencies (Greenwood, Kratochwill, \& Clements, 2008).

Positive Behavioral Intervention Support (PBIS) is the emphasis on school-wide systems of support that include proactive strategies for defining, teaching, and supporting appropriate student behaviors to create a positive school environment (Sailor, Dunlap, Sugai, \& Horner, 2009).

Many districts are now referring to these combined school change initiatives as Effective Behavioral and Instructional Support Systems (EBISS).

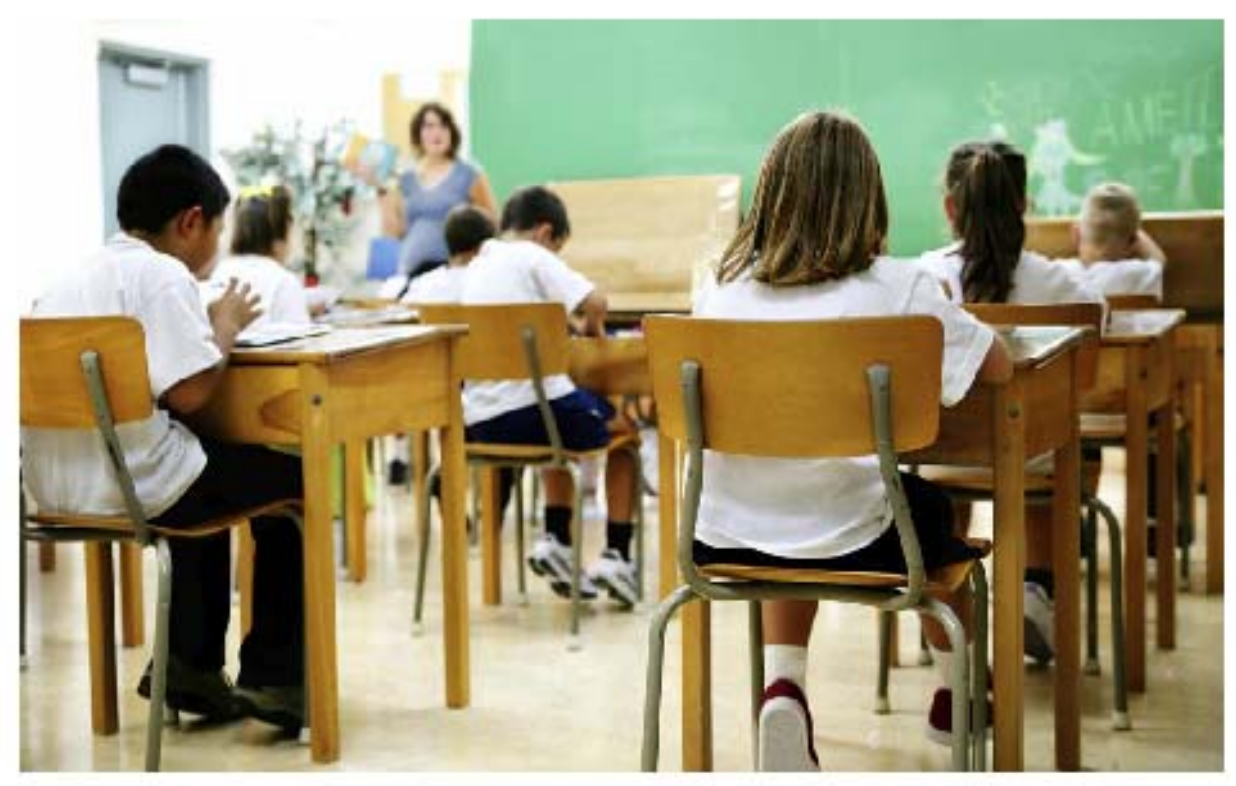




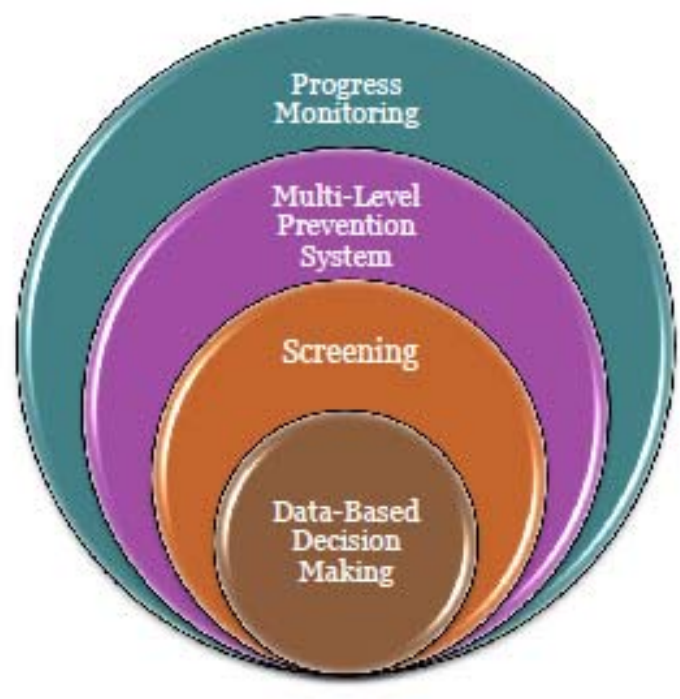

\section{Fiqure 4.}

Essential Components of RTI (adapted from Shinn, Walker, and Stoner, 2002, p. 249)

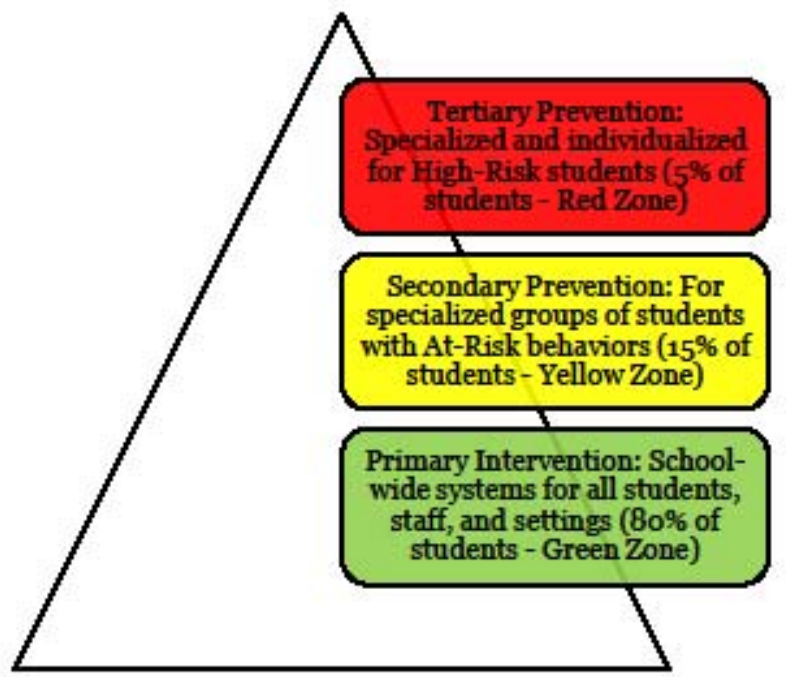

Fioure 5.

PBIS Continuum of School-Wide Support (adapted from Crone \& Horner, 2003, p. 19) 


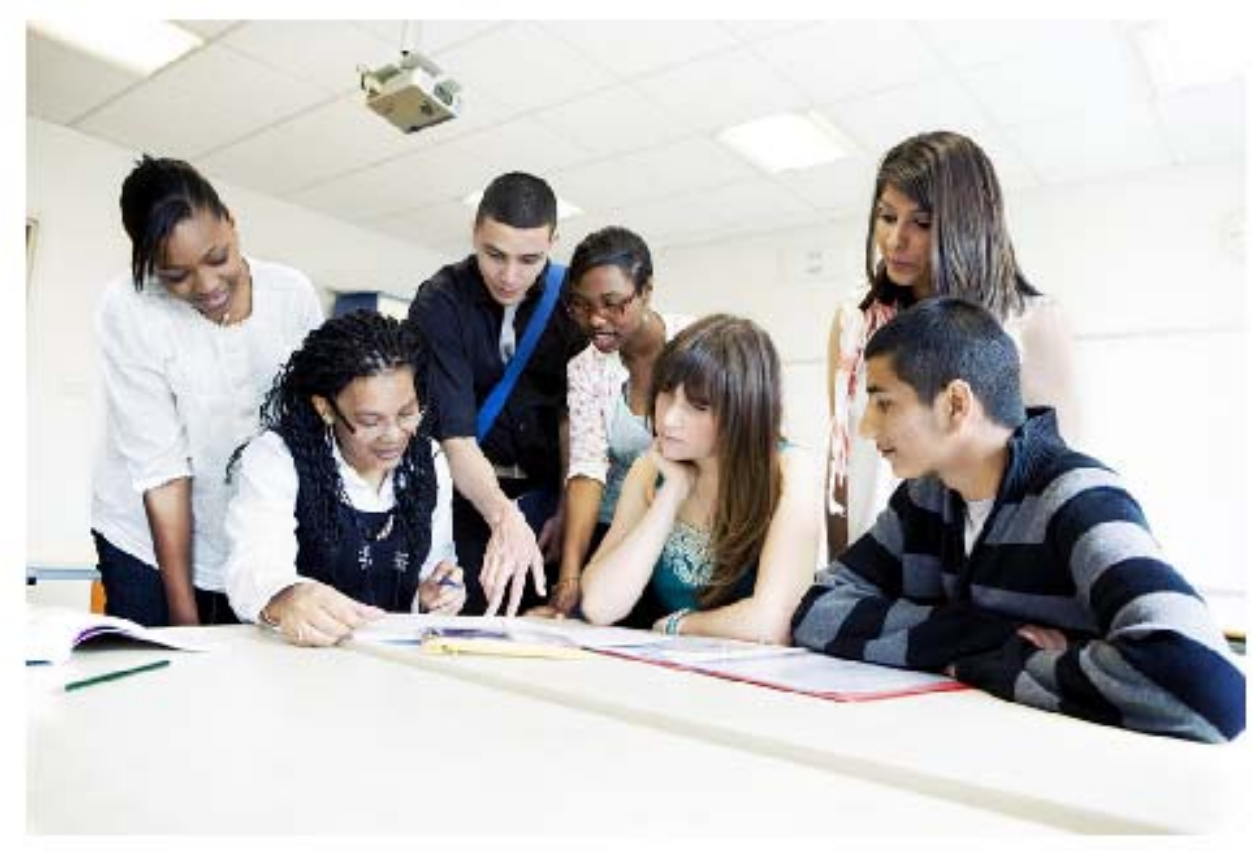

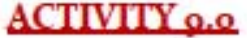

Every school should have a series of interventions in place to help students who are struggling with their academics, behavior, or both. On the pages that follow, there are examples of Pyramids of Intervention. PLCs should review these examples, and then fill out the blank Pyramid of Interventions form (perhaps twice - one for academics and one for behavior). This will help PLCs understand what is available, and perhaps missing, from the school's intervention options. PLCs should bring their pyramids to an all staff meeting. Then, as an entire group, the staff can complete school-wide pyramids. This will help raise awareness for the various interventions that students can access at your school. As you go higher up on the pyramid, the interventions will become more targeted and intensive. 


\section{Pyramid of Interventions}

EXAMPLE for Academics

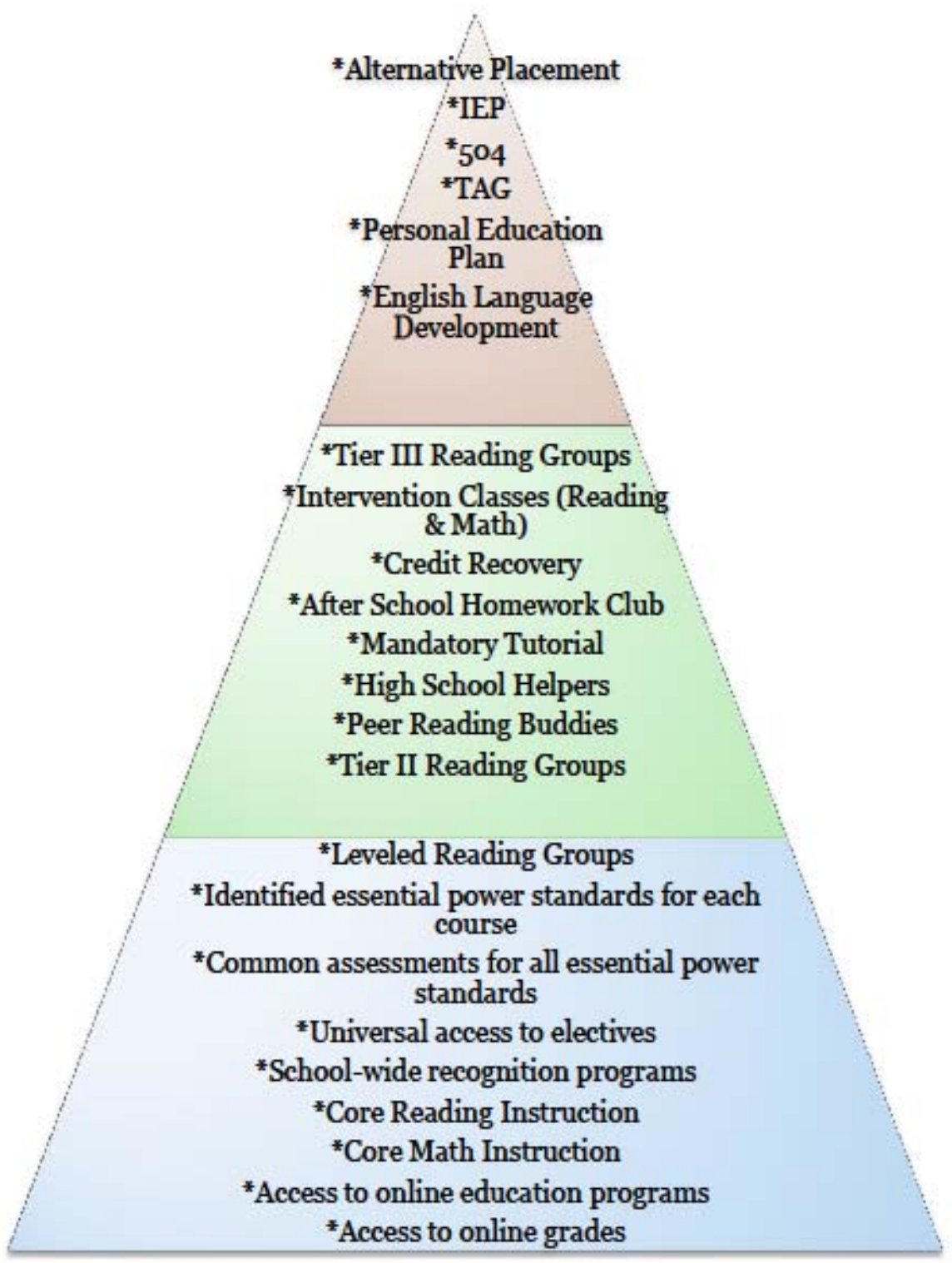




\section{Pyramid of Interventions}

\section{EXAMPLE for Behavior}

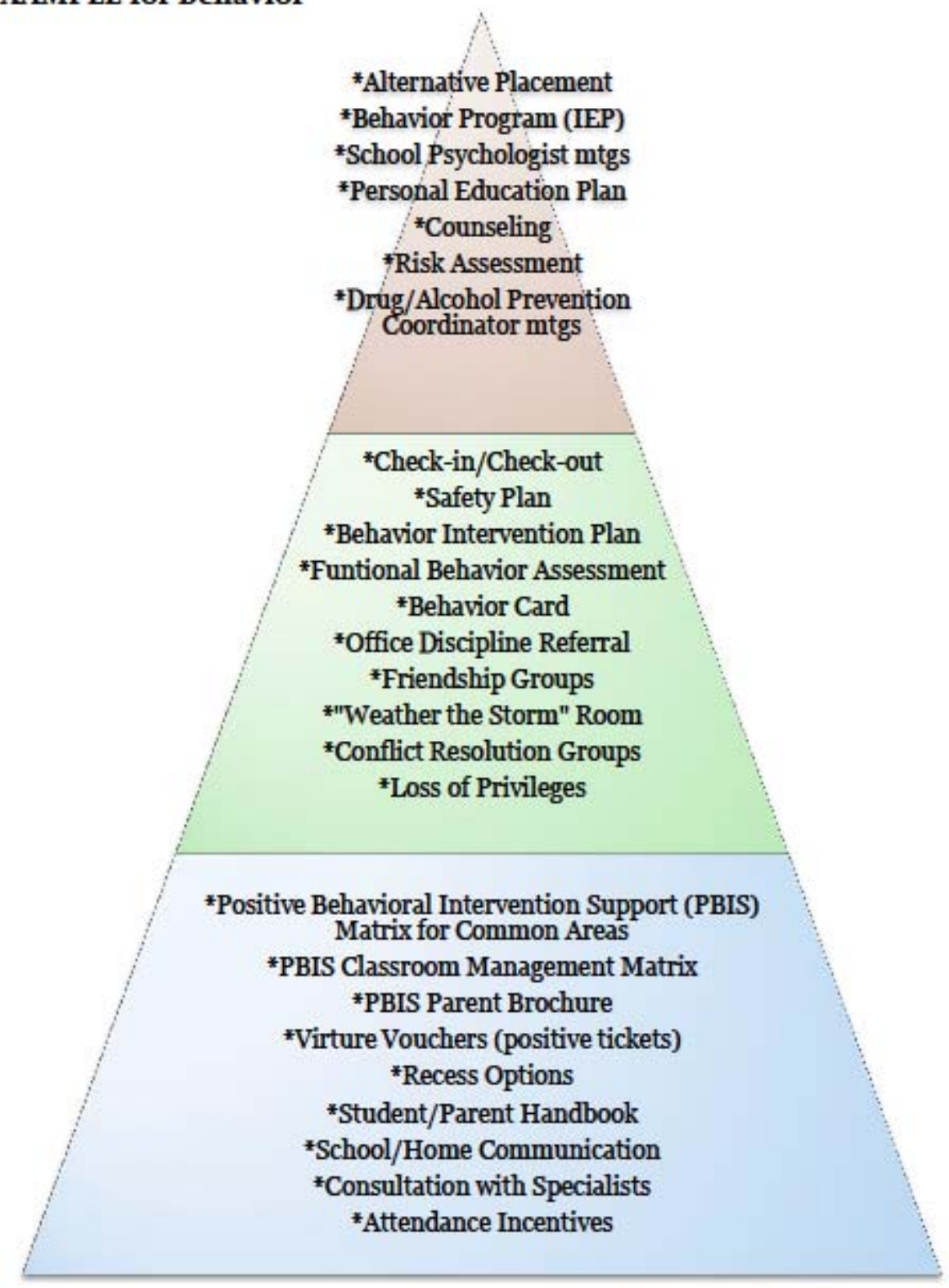




\section{Pyramid of Interventions}

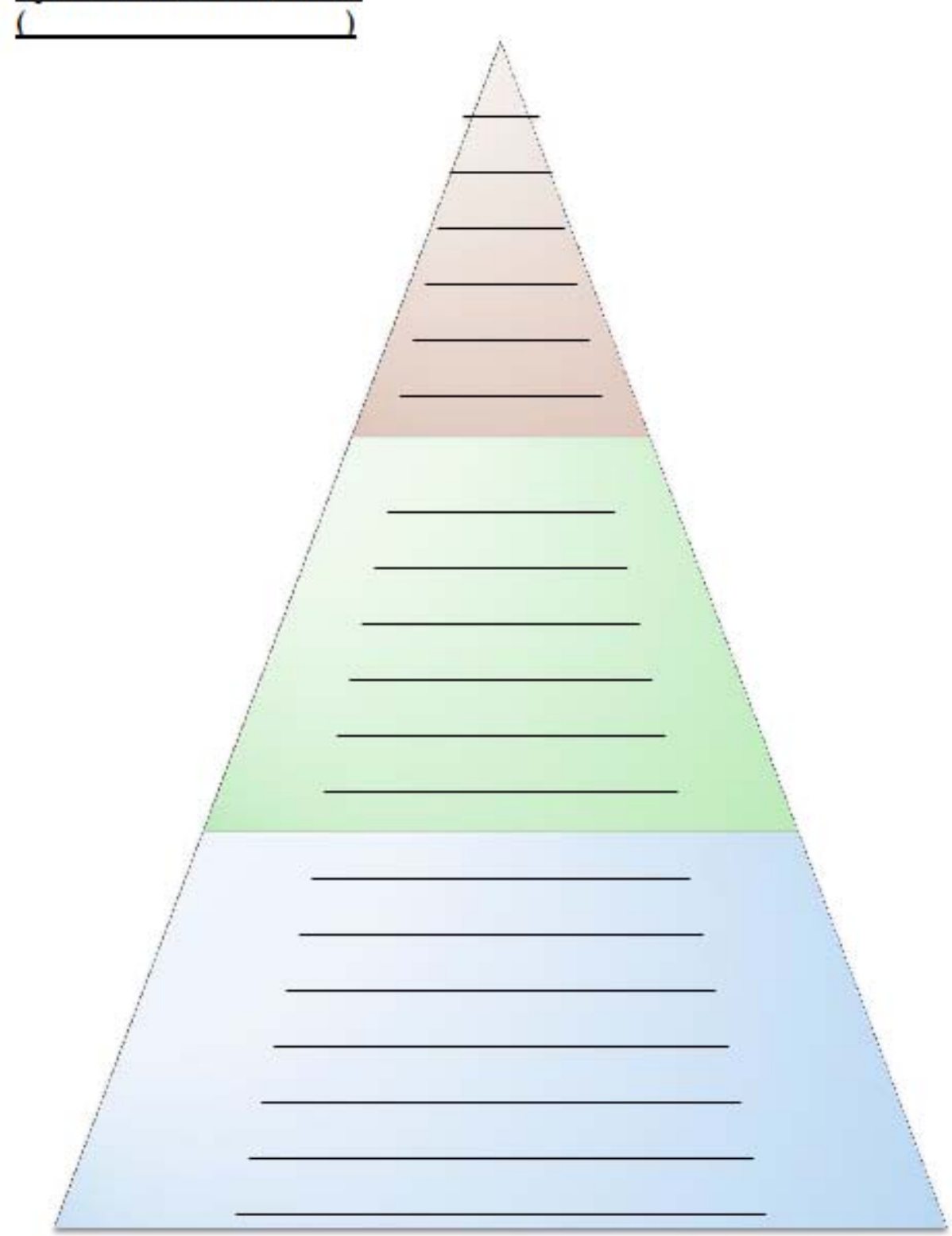




\section{ACTIVITIES 10.0 \& 11.0}

PLCs should analyze the academic and behavioral data for the groups of students they serve. This will help inform them about their areas of strength, as well as areas that need improvement. These activities will lead PLCs to make strategic adjustments in the areas that need improvement. PLCs will be able to refer to their Pyramid of Interventions for Academics \& Behavior to see which interventions are applicable. This activity could also lead to the creation of new interventions not previously listed on the original pyramids. PLCs should complete the Monthly Academic check form, as well as the Monthly Behavior Check form located on the next two pages. Individual teachers should fill the forms out by themselves and bring it to their PLC meeting. Or, PLCs could fill the forms out together. The value will come from the PLC coming together to interact with the data, and exchange ideas regarding how to move forward.

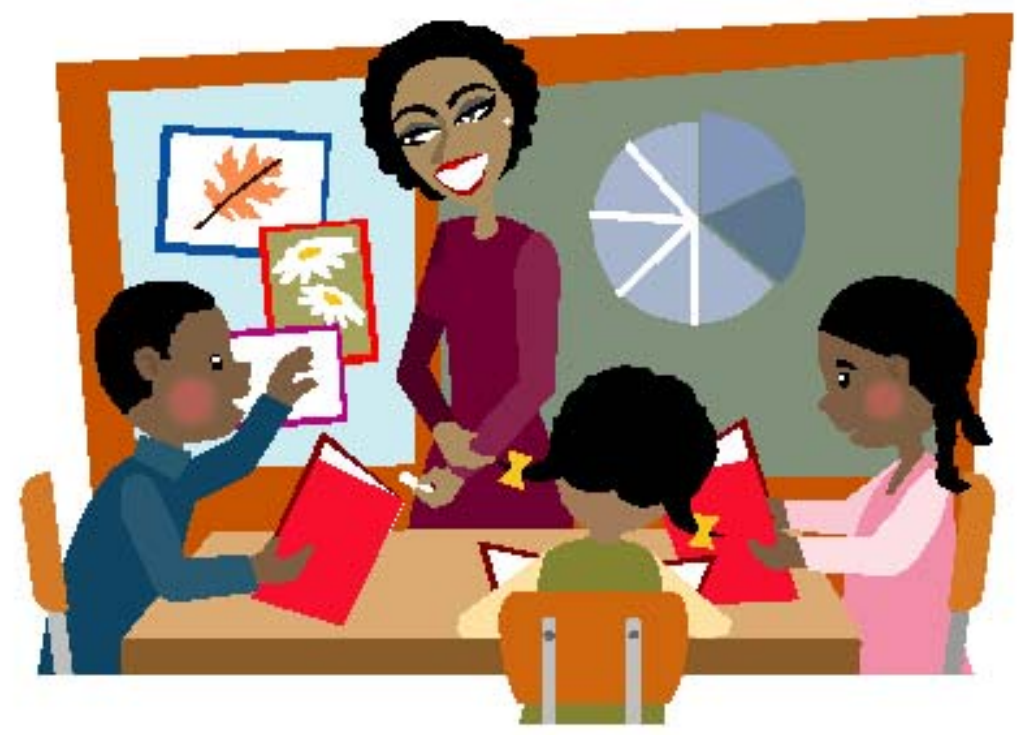




\section{Monthly Academic Check}

PLC:

Date:

What common assessment did you administer?

What skill(s) did the common assessment target?

List the students according to their proficiency level:

\begin{tabular}{|l|l|l|}
\hline Proficient & Nearly Proficient & Not Proficient \\
\hline & & \\
& & \\
& & \\
& & \\
\end{tabular}

What intervention(s) will you use for those who did not demonstrate proficiency?

What enrichment opportunities will you provide for those who demonstrated proficiency and are ready to extend their understanding? 


\section{Monthly Behavior Check}

PLC:

Date:

Answer the following questions using your School-Wide Information System.

How many major discipline referrals did our students accumulate this month?

How many minor discipline referrals did our students accumulate this month?

Which problem behaviors occurred the most this month?

Disrespect Disruption Inappropriate Language Other (explain:

Physical Aggression Dress Code Violation Tardy

Where did most of the problem behaviors occur?

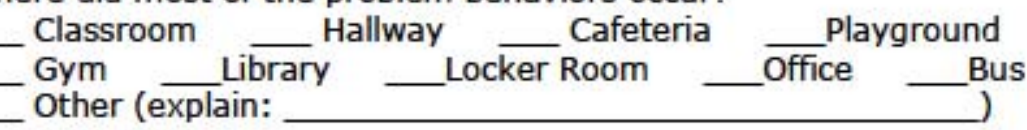

What time did most of the problem behaviors occur?

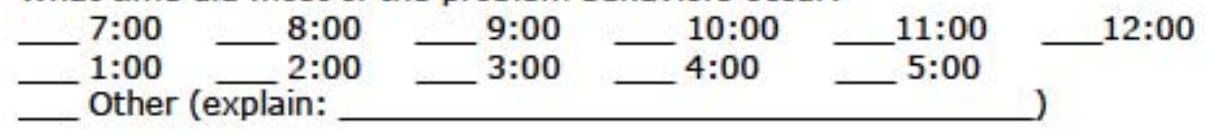

What adjustments (change physical layout, adjust schedules, etc.) can we make as a team to help make the overall situation better?

Are there specific students we are concerned about (list)?

What interventions can we try with the individual students we are concerned about?

How can administration help support you with these individual students? 


\section{How can PLCs support implementing the Common Core State Standards?}

With the adoption of the Common Core State Standards (CCSS), PLCs will need to be well versed in how to communicate changes with staff, students, and parents. Principals need to ensure that teachers and PLCs have support as they implement these new standards. Furthermore, principals need to help teachers and PLCs identify priority standards - the most essential skills and learning targets included in the Common Core State Standards.

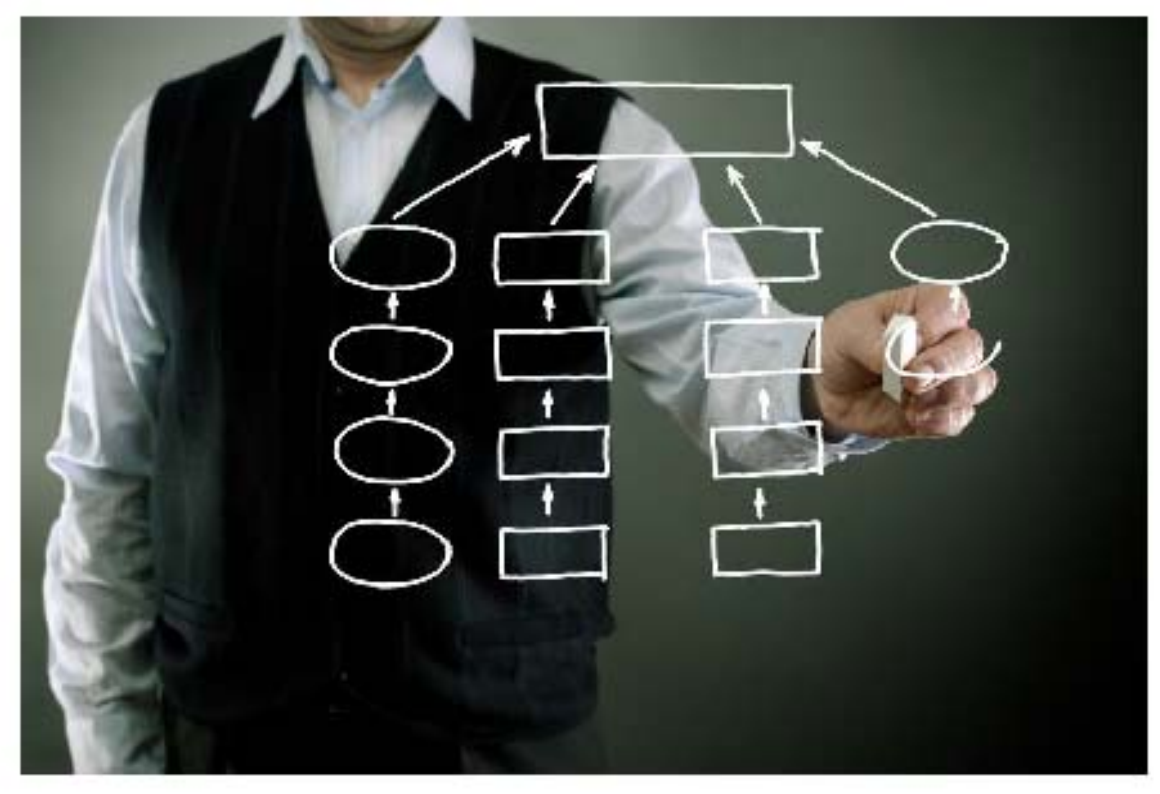

\section{ACTIVITY.2.0}

PLCs should complete the Identifying Priority Standards sheet on the following page. PLCs will be thankful that they are given permission to identify the most essential learning targets from the Common Core State Standards. This activity will empower teachers and give them the freedom to address the priority standards in greater depth. 


\section{Identifying Priority Standards}

PLC: Unit of Study:

${ }^{*}$ Go to www.ode.state or.us/go/commoncore

*Under the Common Core State Standards Implementation Toolkit heading, click on "Teachers"

"Click on "Standards for English Language Arts \& Literacy" or "Standards for Math"

You will either be focusing on "English Language Arts \& Literacy in History/Social Studies, Science, and Technical Subjects" or "Mathematics"

Example of a standard from the Common Core:

2.RI.1 Ask and answer such questions as who, what, where, when, why, and how to demonstrate understanding of key details in a text.

List the standard(s) below that your PLC will be focusing on for this unit of study. These will be your "Priority Standards" for the unit of study.

Fill in the chart below to show how you will address the priority standard(s) in this unit of study.

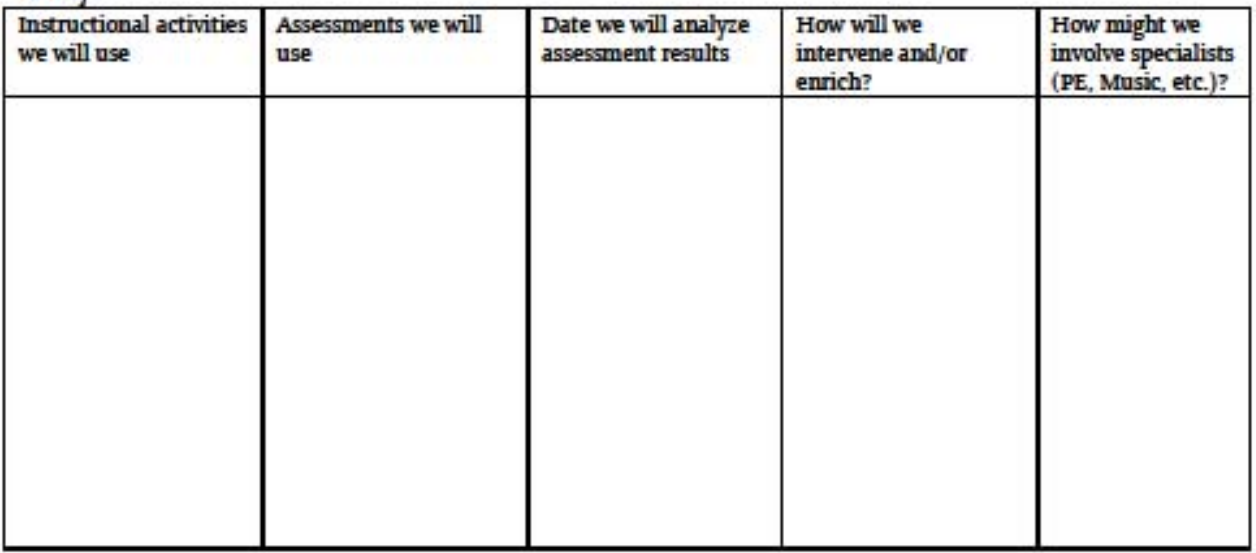




\section{How can PLCs support Proficiency- Based Learning?}

Assessment practices are changing, and principals need to ensure that their PLCs are aware of current best practice. According to the recently adopted House Bill 2220, grades should reflect what a student actually knows. Grades should be based on the mastery of essential skills. Grades should not be inflated due to extra credit. In addition, grades should not suffer due to poor behavior or poor attendance. Grades should provide an accurate picture of what a student knows and can do. The tools in this section will help schools establish guidelines for grading. Having these important discussions in PLCs will help provide consistency throughout the school and district.

One of the most important elements of quality assessment is that students know up front what the learning targets are. Students need to know what it is they are being asked to do. The essential skills and learning targets should not be a mystery. Furthermore, teachers need to ensure that they provide rubrics, which are written in student-friendly language. Rubrics are scoring guides that teachers share with students and parents that are intended to communicate how a student performed on an assessment. It is unreasonable to expect students and parents to wade through educational jargon. Good rubrics allow for quality feedback from the teachers, and guide students toward improving their areas of weakness.

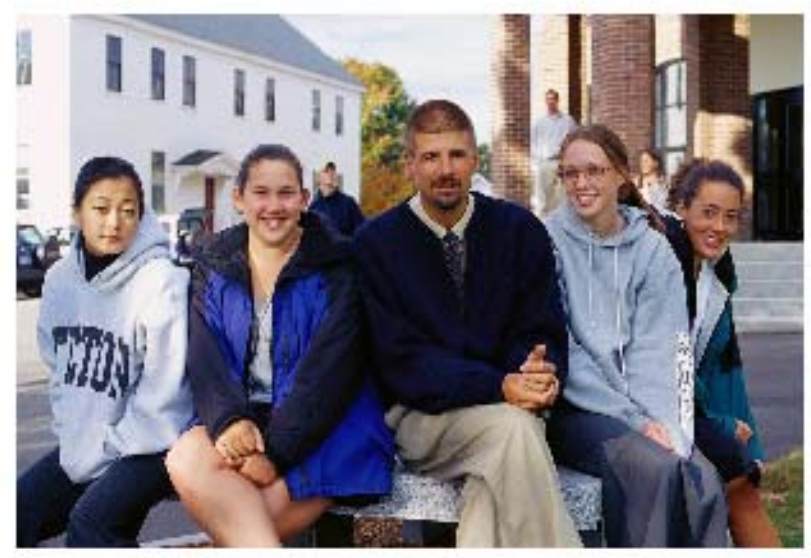


PLCs should be administering common assessments. PLCs should also be analyzing the results of those common assessments in order to plan for interventions and enrichment. Analyzing the data is important so that PLCs can clearly identify the areas where students are weak and strong. When analyzing the results of common assessments, it is not enough to simply look at overall scores. PLCs need to break assessments down into smaller chunks in order to look at specific skills - based on the priority standards the PLC was targeting.

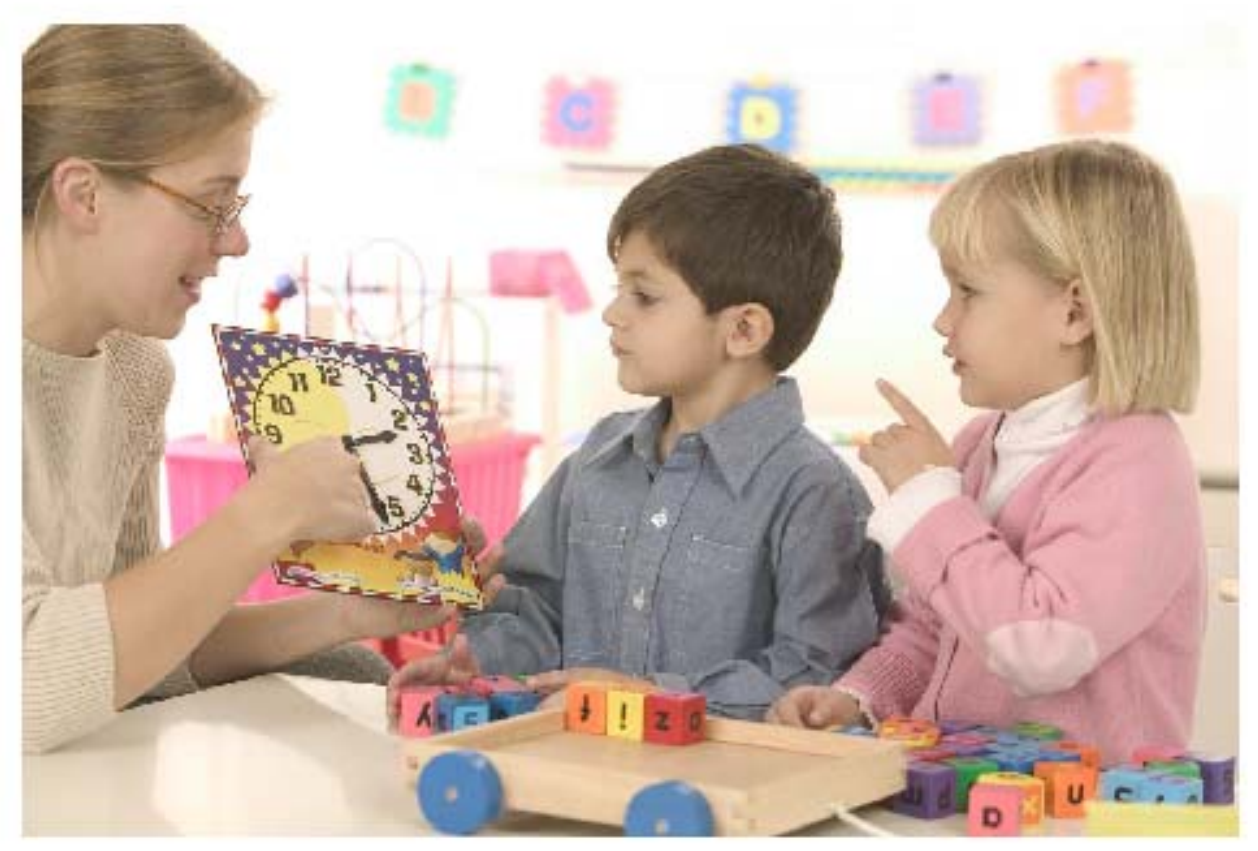

\section{АCTIVITY, 130}

PLCs should complete the AnalyzingYour CommonAssessment form on the following page. Not only will this help PLCs understand where their students are weak and strong, it will also help PLCs understand the strengths and weakness of the assessment they administered. PLCs will get a sense of whether or not the assessment the PLC used needs to be changed, altered, or modified for use again in the future. 


\section{Analyzing Your Common Assessment}

PLC:

Name of Assessment:

Date: Subject Area:

Unit of Study:

Priority Standard(s) Measured:

\begin{tabular}{|l|l|}
\hline Question & Response \\
\hline $\begin{array}{l}\text { What areas did our } \\
\text { students do well on? }\end{array}$ & \\
\hline $\begin{array}{l}\text { What instructional } \\
\text { strategies helped our } \\
\text { students do well? }\end{array}$ & \\
\hline $\begin{array}{l}\text { What skill deficiencies do } \\
\text { we see? }\end{array}$ & \\
\hline $\begin{array}{l}\text { What patterns do we see } \\
\text { in the mistakes, and what } \\
\text { do they tell us? }\end{array}$ & \\
\hline $\begin{array}{l}\text { Which students did not } \\
\text { master the priority } \\
\text { standard(s) and will need } \\
\text { additional time/support? }\end{array}$ & \\
\hline $\begin{array}{l}\text { What intervention will be } \\
\text { provided to address } \\
\text { unlearned skills, and when } \\
\text { will this take place? }\end{array}$ & \\
\hline $\begin{array}{l}\text { Which students mastered } \\
\text { the priority standard(s) } \\
\text { and are ready for } \\
\text { enrichment? }\end{array}$ & \\
\hline $\begin{array}{l}\text { What enrichment } \\
\text { opportunities will be } \\
\text { provided, and when will } \\
\text { they take place? }\end{array}$ & \\
\hline $\begin{array}{l}\text { Do we need to adjust or } \\
\text { improve this assessment? } \\
\text { If so, how? }\end{array}$ & \\
\hline
\end{tabular}




\section{ACTIVITY 14.0}

PLCs should create student-friendly rubrics for the common assessments they administer. PLCs should complete the Creating Student-Friendly Rubrics form located on the next page. This activity will help teachers ensure that they are being clear with their students regarding the expectations for the assessment. The rubrics that are created should be shared with students well before the students are asked to complete the assessment. Also, the rubrics should provide students with helpful feedback that will encourage them to improve their work.

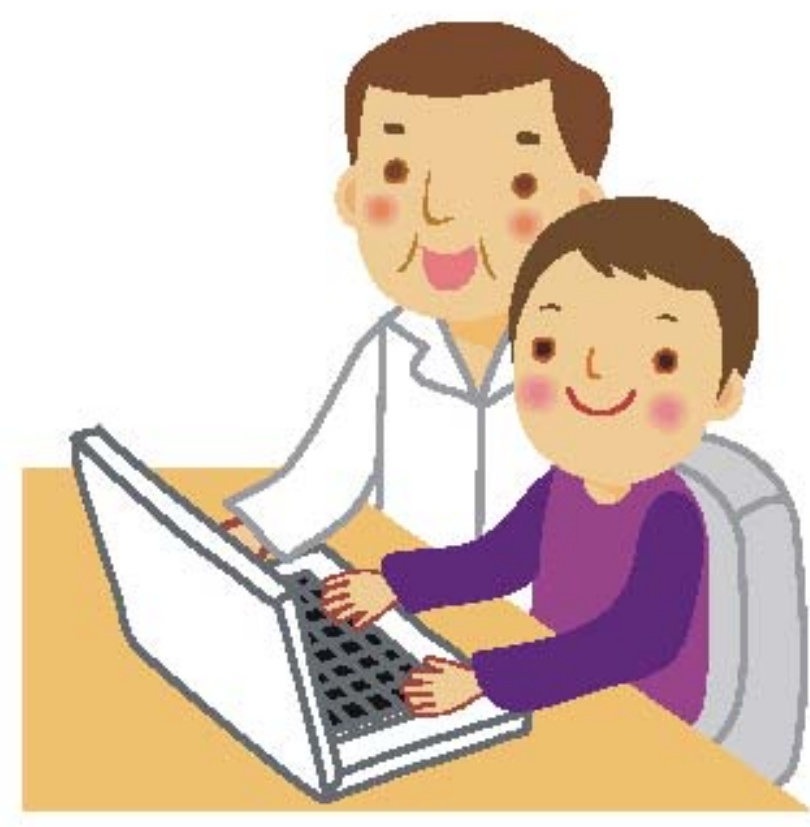




\section{Creating Student-Friendly Rubrics}

What do we want our students to learn? List the priority standard(s) below:

How will we know when they have learned it? What type of common assessment will we use?

What particular skills will we be assessing? How will this be communicated in the rubric we share with students?

What student-friendly terms will we use to help students understand whether or not they mastered the intended skills? (Remember to keep it age appropriate) 


\section{Student-Friendly Rubric EXAMPLE ( $8^{\text {th }}$ grade persuasive writing)}

Writing Prompt: Choose a current political issue and argue for or against it. In your writing, present a clear argument and provide supporting detail. Try to convince your audience to vote/believe the way you do. The power standard being addressed in this assessment is outlined below.

8.W.1 Write arguments to support claims with clear reasons and relevant evidence.

a. Introduce claim(s), acknowledge and distinguish the claim(s) from alternate or opposing claims, and organize the reasons and evidence logically.

b. Support claim(s) with logical reasoning and relevant evidence, using accurate, credible sources and demonstrating an understanding of the topic or text.

c. Use words, phrases, and clauses to create cohesion and clarify the relationships among claim(s), counterclaims, reasons, and evidence.

d. Establish and maintain a formal style.

e. Provide a concluding statement or section that follows from and supports the argument presented.

*Please see the writing scoring rubric on the following page. Your teacher will give you a score for each writing trait. Your teacher will also circle or highlight the areas for each trait that apply to the work sample you submitted. In addition, your teacher will conference with you and give you additional oral feedback (including suggestions for improvement) during Writers' Workshop time. 


\begin{tabular}{|c|c|c|c|c|}
\hline Trait & $\begin{array}{l}\text { 1-Beginning } \\
\text { 2- Emerging }\end{array}$ & 3-Developing & 4-Proficient & $\begin{array}{l}5 \text { - Strong } \\
6 \text { - Exceptional }\end{array}$ \\
\hline $\begin{array}{l}\text { Ideas; The } \\
\text { content; what you } \\
\text { say; your main } \\
\text { idea; subtopics; } \\
\text { supporting details } \\
\text { Score: }\end{array}$ & $\begin{array}{l}\text { Unclear main idea; } \\
\text { not enough details } \\
\text { or information; too } \\
\text { short; repetitive; } \\
\text { confusing ideas; } \\
\text { unoriginal ideas; } \\
\text { hard to } \\
\text { understand }\end{array}$ & $\begin{array}{l}\text { Not quite enough } \\
\text { information or } \\
\text { details; confusing; } \\
\text { too general; too } \\
\text { predictable; lacks } \\
\text { originality; off } \\
\text { topic }\end{array}$ & $\begin{array}{l}\text { Clear main idea; } \\
\text { support is present; } \\
\text { focused; on topic; } \\
\text { ideas are well } \\
\text { developed; specific } \\
\text { details support } \\
\text { main idea }\end{array}$ & $\begin{array}{l}\text { Interesting } \\
\text { connections and } \\
\text { insights; main } \\
\text { ideas stand out; } \\
\text { strong support; } \\
\text { rich details; holds } \\
\text { reader's attention }\end{array}$ \\
\hline $\begin{array}{l}\text { Organization: } \\
\text { The order in which } \\
\text { your ideas are } \\
\text { placed; beginning, } \\
\text { middle, and end; } \\
\text { paragraphing } \\
\text { Score: }\end{array}$ & $\begin{array}{l}\text { No introduction or } \\
\text { conclusion; lacks } \\
\text { paragraph breaks; } \\
\text { very confusing; } \\
\text { hard to follow; } \\
\text { requires re- } \\
\text { reading }\end{array}$ & $\begin{array}{l}\text { Intro/conclusion } \\
\text { needs developing; } \\
\text { illogical order; } \\
\text { weak transitions; } \\
\text { unbalanced; needs } \\
\text { paragraph breaks }\end{array}$ & $\begin{array}{l}\text { Clear beginning, } \\
\text { middle, and end } \\
\text { (intro, body, and } \\
\text { conclusion); } \\
\text { sequenced ideas; } \\
\text { transition words } \\
\text { used }\end{array}$ & $\begin{array}{l}\text { Inviting beginning; } \\
\text { satisfying } \\
\text { resolution; } \\
\text { effective sequence, } \\
\text { strong transitions, } \\
\text { easy to follow }\end{array}$ \\
\hline $\begin{array}{l}\text { Voice: The words } \\
\text { used to give the } \\
\text { reader an idea of } \\
\text { the writer's } \\
\text { personality and } \\
\text { commitment to the } \\
\text { topic }\end{array}$ & $\begin{array}{l}\text { Little hint of the } \\
\text { writer behind the } \\
\text { words; lifeless; flat } \\
\text { writing; topic } \\
\text { doesn't come to } \\
\text { life; writer doesn't } \\
\text { seem committed }\end{array}$ & $\begin{array}{l}\text { Limited sense of } \\
\text { audience; robotic } \\
\text { or mechanical; too } \\
\text { casual or too } \\
\text { formal; somewhat } \\
\text { committed to topic }\end{array}$ & \begin{tabular}{|l|} 
Sincere; \\
humorous; \\
enthusiastic; \\
energetic; lively in \\
places; sense of \\
audience; \\
committed to topic
\end{tabular} & $\begin{array}{l}\text { Engaging; } \\
\text { expressive; lively; } \\
\text { passionate; strong } \\
\text { personal voice; } \\
\text { comes to life; } \\
\text { highly committed }\end{array}$ \\
\hline Score: & & & & \\
\hline $\begin{array}{l}\text { Word Choice: } \\
\text { Selecting a variety } \\
\text { of lively words } \\
\text { appropriate to } \\
\text { audience and } \\
\text { purpose } \\
\text { Score: }\end{array}$ & $\begin{array}{l}\text { Extremely } \\
\text { ordinary and } \\
\text { boring words; } \\
\text { absent of images; } \\
\text { words frequently } \\
\text { used incorrectly; } \\
\text { little variety in } \\
\text { words }\end{array}$ & $\begin{array}{l}\text { Ordinary, common } \\
\text { words or phrases, } \\
\text { too technical; } \\
\text { misused words; } \\
\text { needs more lively } \\
\text { verbs, adjectives }\end{array}$ & $\begin{array}{l}\text { Variety of words } \\
\text { that work; } \\
\text { attempts at } \\
\text { colorful language; } \\
\text { strong verbs; } \\
\text { precise adjectives }\end{array}$ & $\begin{array}{l}\text { Words are } \\
\text { carefully chosen; } \\
\text { clear images; } \\
\text { precise, vivid, } \\
\text { lively, and } \\
\text { descriptive words }\end{array}$ \\
\hline $\begin{array}{l}\text { Sentence } \\
\text { Fluency: The flow } \\
\text { of the writing } \\
\text { created by the } \\
\text { variety and pattern } \\
\text { of sentences } \\
\text { Score: }\end{array}$ & $\begin{array}{l}\text { Difficult to read; } \\
\text { choppy, rambling, } \\
\text { awkward } \\
\text { sentences; } \\
\text { fragments; lacks } \\
\text { variety in } \\
\text { sentences }\end{array}$ & $\begin{array}{l}\text { Little variety; } \\
\text { awkward sentence } \\
\text { construction; run- } \\
\text { on sentences; not } \\
\text { much flow; } \\
\text { difficult to read }\end{array}$ & $\begin{array}{l}\text { Good flow; variety } \\
\text { of sentence } \\
\text { structures, } \\
\text { beginnings and } \\
\text { lengths; easy to } \\
\text { read aloud }\end{array}$ & $\begin{array}{l}\text { Natural; enjoyable } \\
\text { flow and rhythm; } \\
\text { variation that adds } \\
\text { interest; control } \\
\text { over sentence } \\
\text { structure }\end{array}$ \\
\hline $\begin{array}{l}\text { Conventions: } \\
\text { Grammar, } \\
\text { punctuation, } \\
\text { capitalization, } \\
\text { spelling, sentence } \\
\text { structure, tenses, } \\
\text { and usage } \\
\text { Score: } \\
\end{array}$ & $\begin{array}{l}\text { Little control of } \\
\text { written language; } \\
\text { errors distract } \\
\text { reader; many } \\
\text { errors make it } \\
\text { difficult to read; } \\
\text { needs a lot of } \\
\text { editing }\end{array}$ & $\begin{array}{l}\text { Spelling, grammar, } \\
\text { punctuation, } \\
\text { capitalization, } \\
\text { sentence structure } \\
\text { need editing or } \\
\text { affect readability }\end{array}$ & $\begin{array}{l}\text { Control over most } \\
\text { things; mostly free } \\
\text { of significant } \\
\text { errors; mostly } \\
\text { correct spelling; } \\
\text { only minor editing } \\
\text { needed }\end{array}$ & $\begin{array}{l}\text { Strong control of } \\
\text { written language; } \\
\text { wide skill range; } \\
\text { little or no need for } \\
\text { editing }\end{array}$ \\
\hline
\end{tabular}


Regarding Proficiency-Based Learning, sometimes it is not that a student "can't" do it; it is that they "won't" do it. Sometimes, students know the essential learning targets, but they are not doing anything to demonstrate their proficiency. When this situation applies, PLCs must have a way to hold students accountable and communicate with parents regarding the expectations for the class.

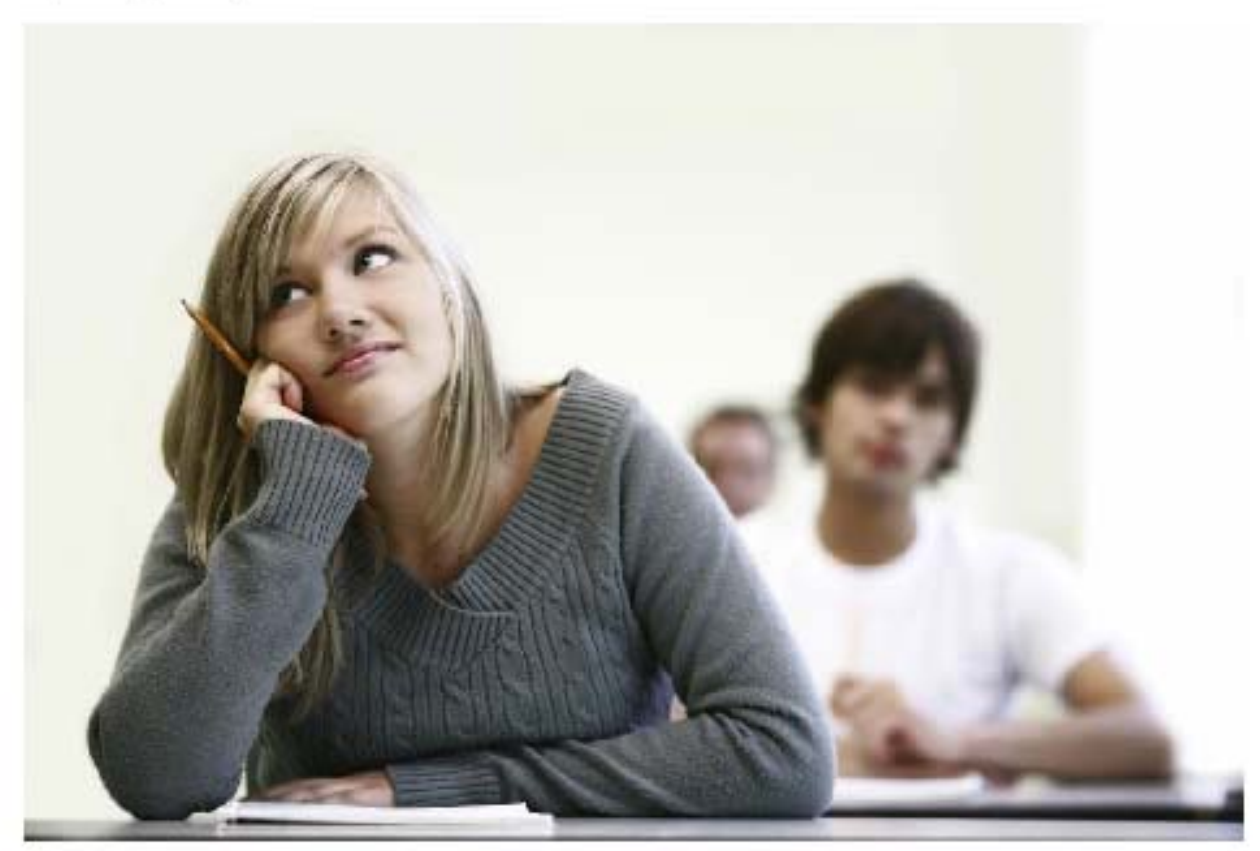

\section{ACTIVITY 15.0}

PLCs should review the Incomplete Contract Agreement on the next page. PLCs may choose to modify the form (you need to do what works best for your school). Also, adjustments may be needed to make it age appropriate. However, it is imperative to have something like this in place so that PLCs can get an accurate picture of whether or not students are proficient. 


\section{Incomplete Contract Agreement}

Student Name:

Course Title:

Date:

Why I am not completing my work on time and therefore cannot demonstrate proficiency of the essential skills:

Date I will complete my work and demonstrate proficiency:

(This cannot be more than three weeks after the original due date unless you have been absent for a substantial amount of time)

Student Signature:

Date:

My signature acknowledges that I will receive an $\mathrm{N}$ (no grade) if I do not meet the new deadline. I further understand that I would then have to meet the learning standard on a different assessment. I understand that this different assessment may mean greater effort for me, so I give my word to complete the original assessment by the new deadline. I also understand that not completing work in a timely fashion will negatively affect my "personal management grade" and make it more difficult to obtain my goal of earning a passing academic grade in this course.

Teacher acceptance of the above agreement:

Teacher Signature: Date: 
DISTRICT/COMMUNITY COIT_ABORATION - The activities included in the rest of this handbook refer to overlapping common interests between the district and the community. A number of mutually beneficial initiatives emerged through the PLC process during the 201314 school year. The handbook activities that follow are intended to help continue and enhance the collaborative partnerships that exist between the district and community.

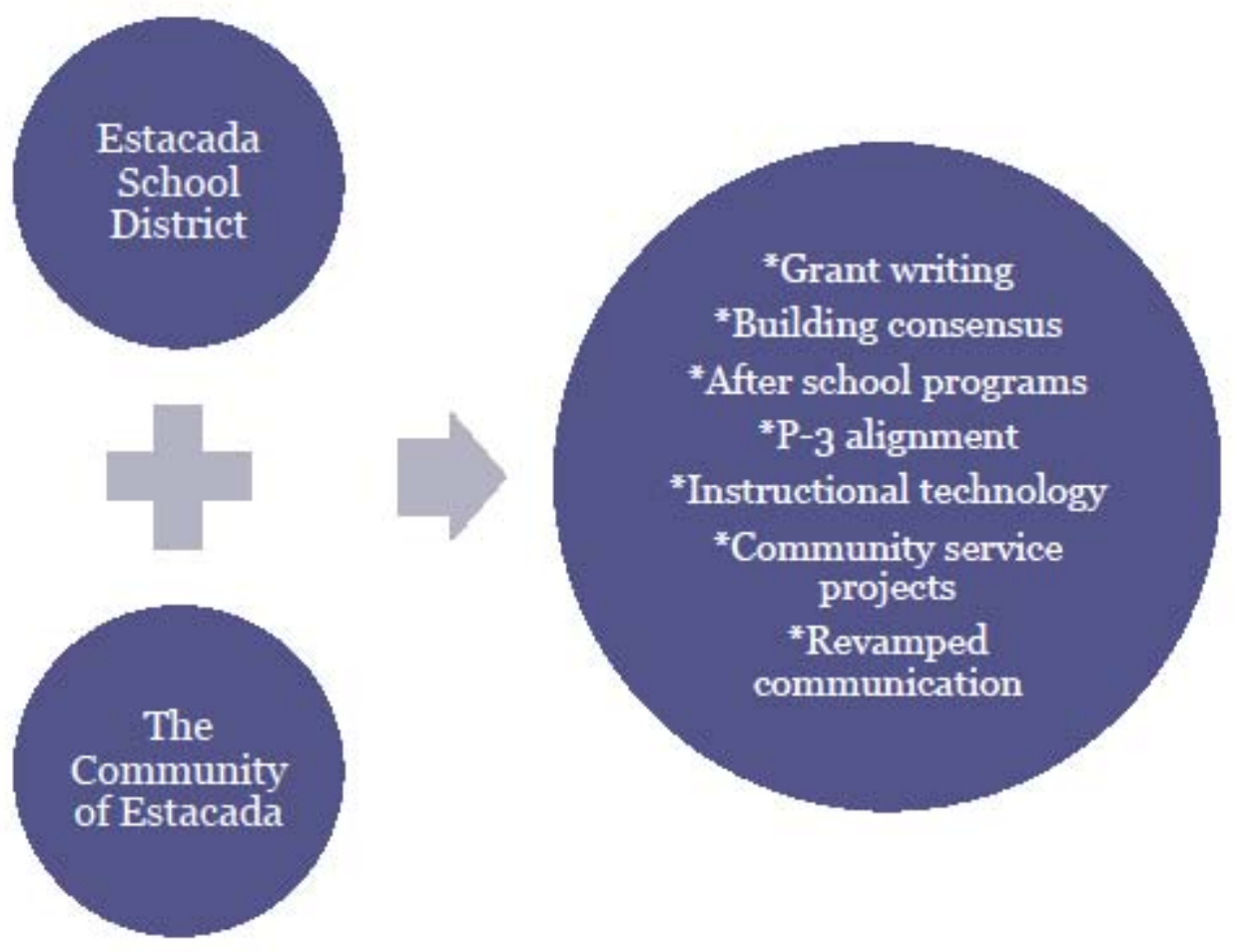

Figure 6.0

Common Interests of the Estacada School District \& Community of Estacada 


\section{How Can PLCs Involve Families \& Community Members?}

A number of initiatives have emerged in Estacada as a result of PLCs collaborating with families and community members. These initiatives include:

- Grant writing

- Building consensus

- After school enrichment programs

- P-3 alignment

- Instructional technology

- Community service projects

- Revamped communication between the district and community

On the pages that follow, you will find activities designed to move these initiatives forward and increase collaboration between school staff and family/community members through the PLC process.

\section{ACTIVITH.16.0}

On the next page, you will find resources regarding the demographics of Estacada. Most grant request for proposals (RFPs) will require demographics information. Without the right tools, gathering this information can be difficult and time consuming, which could halt PLC grant writing efforts before they even begin. The Grant Writing: Getting Started activity on the next page will allow PLCs to easily locate demographics information required for grant writing. 


\section{Grant Writing: Getting Started}

Go to www.oregonexplorer,info/pural

The Oregon Bural Communities Explorer tool is a public, unbiased information systom and toaching tool for rural leaders. This tool will provide you with access to:

- Community data

- Rural rasearch

- Community stories and local reports contributed by Oregon residents

- Historical documents and community visions contributed by Oregón residents

- Additional resourcess and portals

*Follow the tutorial to got started. Then, simply plug in "Rstacada" along with all of the various data points you would like to find. This tool will halp you complate the "demographios" portion for the grants you are interested in applying for. 


\section{ACTIVITY $_{17.0}$}

The Building Consensus activity provides a number of options that will help PLCs (or Parent Organizations) build consensus when working on shared projects (including grant writing). Consensus is mutual agreement among team members that all legitimate concerns of individuals have been addressed by the group and everyone agrees to support the decision. Consensus means that all members have had an opportunity to express their opinions and feel that they have been listened to by the group. Team members don't all have to think that the decision is the best decision, but they all agree that they can live with it. Before coming to consensus, it is important that team members first come to a place of understanding and agreement.

\section{Building Consensus}

PLCs may use the following techniques to achieve understanding:

- Post and review an agenda so that all group members know what is to be discussed and decided.

- Brainstorm to encourage creative thinking regarding options.

- Gather information from everyone present using the Round Robin method (so that all members are heard, and quieter team members are "forced" to share their ideas)

- Discuss further, and perhaps use the Tallking Stick method (only the person holding the stick gets to talk) so that team members can share their thoughts without being cut off or interrupted.

PLCs may use the following techniques to achieve agreement:

- Poll the group for opinions to see how close the group is to agreement.

- Identify the Pros \& Cons associated with the emergent project ideas. 
- After discussing the Pros \& Cons, create a chart that includes the main project ideas. Post Self-Stick Dots to determine the group's priorities. Each group member should be allowed multiple SelfStick Dots (depending on the number of project ideas that are posted - three dots per person is usually a desirable number). Each person uses all of their allotted dots (to identify their top choices). The ideas that have the most dots next to them should be the ideas that are pursued further.

- Once the team members have seen the ideas that had the most dots, they will get a better sense of the ideas most people will support. Next, PLCs may use the PRES model to provide more clarification. For the PRES model, each member will write down the following:

- Roint: What you want to have happen

- Reason: Why you think it's a good option

- Example: Where has option been successful elsewhere?

- Summary: Recap what you support and why

- PLCs may then use the Fist to Five method. Group members will indicate with their hand/fingers where they are with the main project idea(s).

- Fist (no fingers) means NO - I cannot live with this.

- 1 finger means WAIT - There are issues to discuss.

- 2 fingers mean I'M CLOSE - I have small concerns.

- 3 fingers mean $\mathrm{OK}-\mathrm{I}$ can live with this decision.

- 4 fingers mean YES - It is a good decision. I will work for it.

- 5 fingers mean YES - It is a great decision. I will be a leader in implementing it. 


\section{ACTIVITY 18.0}

PLCs worked to create an after school program for the district based on the acronym STEAM (Science, Technology, Engineering, Arts, and Math). This program has been built around the local experts in each area who have been willing to volunteer their time. In order to sustain (and build) the program, we will need to continue to reach out to our community. PLCs (and parent/community volunteers they work with) should complete the grid below so that we can continue to recruit local talent for our after school enrichment program. Thank you for helping us move forward - Full STEAM Ahead!

\section{After School Enrichment Program}

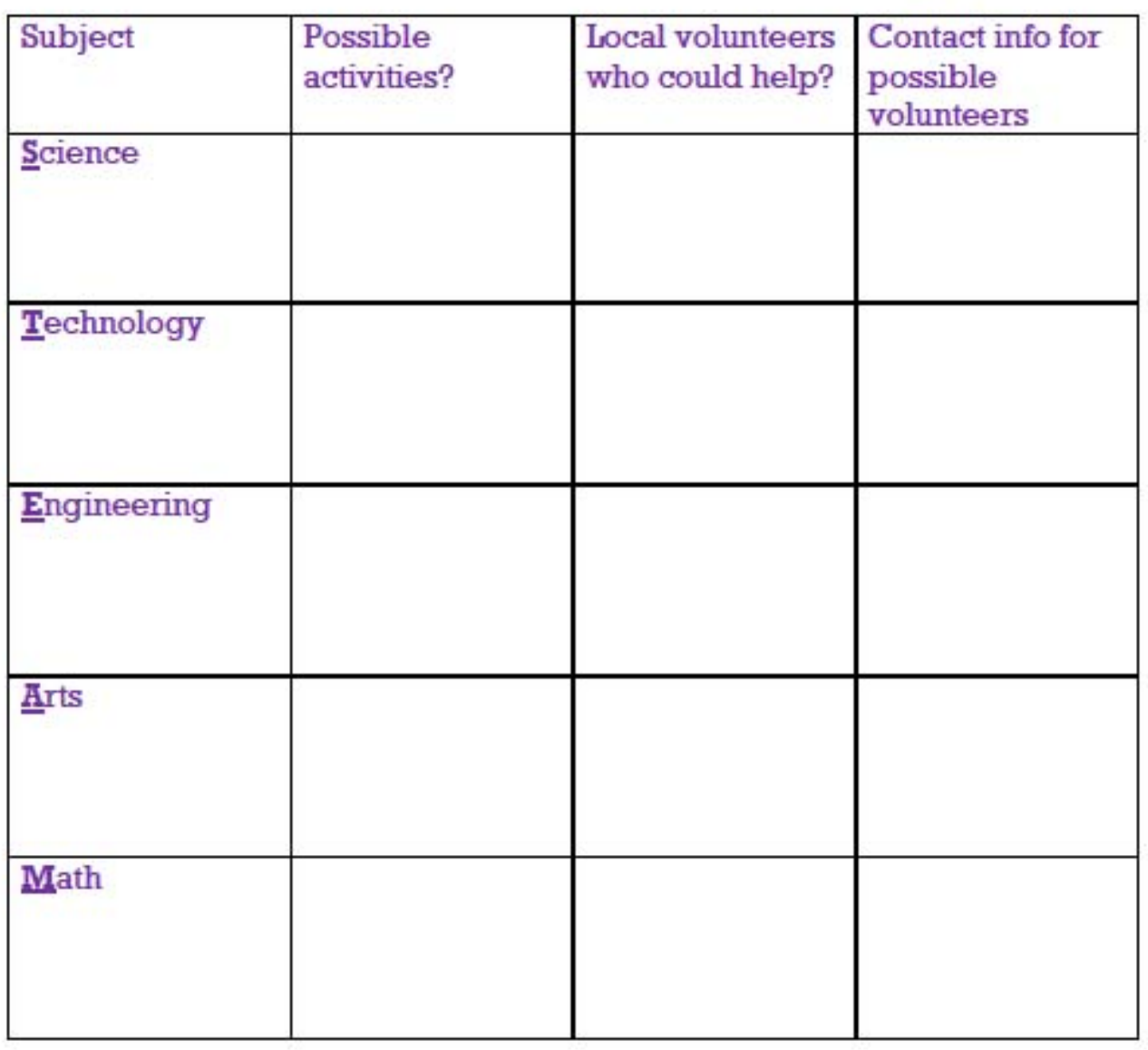




\section{ACTIVITY 19.0}

An initiative that emerged from the PLC process during the 2013-14 school year was the desire to emphasize $\mathrm{P}-3$ (prenatal $-3^{\mathrm{nt}}$ grade) alignment. This initiative is important because it connects the district to our community more deeply, specifically with expectant mothers/families, area preschools, and local child care providers. Outcomes include a heightened awareness of what it means to be "Kindergarten-ready" (as indicated by the Common Core State Standards). Another desired outcome is to have all students reading at grade level by $3^{\text {rt }}$ grade. An Estacada PLC applied for a \$225,000 grant to support this effort. We will find out in February if we received the grant. However, this initiative must move forward with or without the grant. Regardless of the outcome of the grant, the application process allowed the lead PLC to engage in systematic planning around the initiative. These key concepts will need to be revisited on a regular basis as the initiative moves forward. See what your PLC can contribute by completing the activity below.

\section{Planning For P-3 Alignment}

\section{A. PLANNING / PROJECT DEVELOPMENT ISSUES}

1. What is the goal/desired outcome from implementation of $\mathrm{P}-3$ alignment strategies?

2. Who will be actively involved in the alignment project? Please detail individuals and entities they represent. What relationships already exist, and which need development?

\begin{tabular}{|l|l|l|}
\hline Who & Existing Relationship & Needs development, how? \\
\hline & & \\
\hline & & \\
\hline & & \\
\hline & & \\
\hline & & \\
\hline
\end{tabular}


3. What barriers or challenges do we foresee in this work?

B. ACTIONS TO BE TAKEN IN THE FIRST YEAR

By "Buckets" from the Kauerz/Coffman Framework:

1. Cross-sector Work

2. Administrator Effectiveness

3. Teacher Effectiveness

4. Instructional Tools

5. Learning Environment

$\bar{\square}$

6. Data-driven Improvement

7. Family Engagement 
8. Continuity \& Pathways

\section{NUJIBERS NEEDED (annual for each of vears 1-3)}

\section{Child}

- Number of children receiving high quality Early Childhood Education

- Number of children receiving transition services

- Number of children receiving early intervention services and support in kindergarten or preschool

\section{Family}

- Number and type of programs for families to support children's learning \& literacy development

- Number of resources created to communicate with families (flyers, presentations, etc.)

- Number of families participating in family support activities

\section{Educators}

- Number of educators trained in shared professional development

- Number and type of meetings between Early Childhood Education and elementary educators

- Results of the P-3 Summit (held on October 14, 2013) 
D. p-3 Alignment Required Tasks

\begin{tabular}{|l|l|l|}
\hline \multicolumn{1}{|c|}{$\begin{array}{c}\text { Info / Data needed for P-3 Alignment } \\
\text { Requirements }\end{array}$} & Assigned to & $\begin{array}{c}\text { Date to be } \\
\text { completed }\end{array}$ \\
\cline { 2 - 3 } & & \\
\hline $\begin{array}{l}\text { Describe the district applying for this opportunity, } \\
\text { including demographic information for elementary } \\
\text { schools. }\end{array}$ & & \\
\hline Document achievement gap (stats, grades, etc.) & & \\
\hline $\begin{array}{l}\text { Describe any current or past efforts to strengthen } \\
\text { connections with early care and learning and families. }\end{array}$ & & \\
\hline $\begin{array}{l}\text { Describe the leadership in place to achieve the goals of } \\
\text { the initiative. } \\
\text { Numbers (for Part c) }\end{array}$ & & \\
\hline Child outputs & & \\
\hline Family outputs & & \\
\hline Educators outputs & & \\
\hline Systems Development & & \\
\hline $\begin{array}{l}\text { Elements of kindergarten transition plan \& how it will } \\
\text { be implemented }\end{array}$ & & \\
\hline $\begin{array}{l}\text { Development of data sharing agreements and plans: } \\
\text { process and responsible parties }\end{array}$ & & \\
\hline $\begin{array}{l}\text { Assignment of, and process for: } \\
\text { Completion of, and regular updates to, community } \\
\text { needs assessments and mapping of services }\end{array}$ & & \\
\hline $\begin{array}{l}\text { Identification of adequate resources used to deliver } \\
\text { reform }\end{array}$ & & \\
\hline $\begin{array}{l}\text { Leadership team established (by when, composition) } \\
\text { Professional development for administrators on }\end{array}$ & & \\
\hline
\end{tabular}




\begin{tabular}{|l|l|l|}
\cline { 2 - 3 } \multicolumn{1}{|c|}{ Needed Documents } & \\
\hline $\begin{array}{l}\text { Proposed three-year project budget, identifying } \\
\text { revenue (cash and in-kind) sources and expenditures } \\
\text { for the P-3 project in a detailed line-item format. }\end{array}$ & & \\
\hline Organization budget for the current fiscalyear & & \\
\hline $\begin{array}{l}\text { List of District Board(s), with affiliations and phone } \\
\text { numbers }\end{array}$ & & \\
\hline Endorsement letter from the district superintendent. & & \\
\hline $\begin{array}{l}\text { Letters from principal(s) to be involved with this } \\
\text { project, outlining anticipated roles and interest in this } \\
\text { work. }\end{array}$ & & \\
\hline $\begin{array}{l}\text { Letters of support from partners that outline } \\
\text { partnership plans, commitment to collaboration and } \\
\text { the resources brought to the table (relationships, in- } \\
\text { kind and/or monetary). }\end{array}$ & & \\
\hline
\end{tabular}

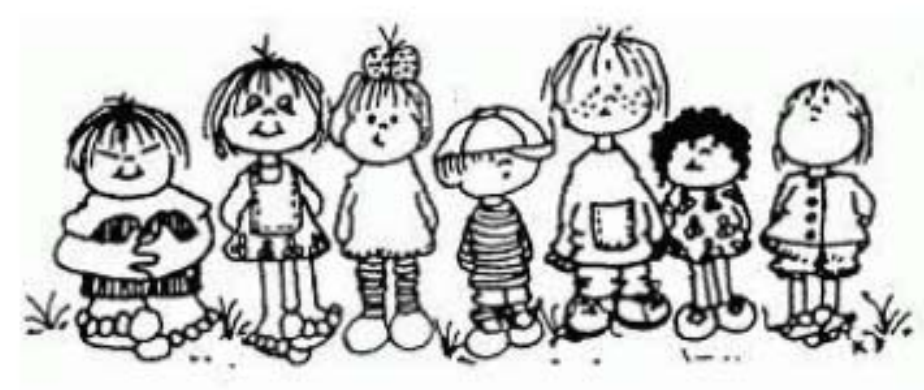




\section{ACTIVITY 20.0}

Another initiative that emerged from PLC work during the 2013-14 school year was a desire to infuse more instructional technology into the classrooms of our schools. A district PLC partnered with our charter schools to attend the iNACOL conference, which is a well-recognized national conference that focuses on instructional technology. From that experience, the district has started looking at ways to acquire grants for funding that support this effort. Eventually, we would like to be a one-to-one school district (one educational device for each student in the district). iNACOL suggested focusing on the early adopters of the district (teachers/staff who are enthusiastic about using instructional technology in the classroom). Would your PLC like to pilot instructional technology programs for the district so that we can further evaluate the best tools to use with our students? If so, please complete the Technology Mini Grant Application below.

\section{Technology Mini Grant Application}

Name

Date

Building Position

I am applying for the following:

An In-Focus Projector

A document camera
A Promethean board

Other (please specify) 
List any other technology-related items that you would like to request for your classroom.

1.

2

5.

Please give a brief outline of a lesson plan showing how you would incorporate this technology.

\section{TRAINING}

The Technology Department would like to provide training that fits the needs of district staff members. If you would like training for any item that you are requesting on this application, please indicate that below. You may also request training for hardware and software that you use as a normal part of your job.

FPlease return this form to your building technology coordinator. 


\section{ACTIVITY 21.0}

The district has discovered that there are a number of local organizations (Todos Juntos, Estacada Together, Estacada Community Foundation, etc.) who would like to partner with the school district to work on mutually beneficial community service projects through grants. Below, you will find a sample Memorandum of Understanding fora Fiscal Partner Relationship form. While we would like your PLC to always run these through an administrator, much of the planning and collaboration you have done with these local organizations can be captured in this form. In many cases, it is best to have the organization you are partnering with handle to fiscal aspects of the grant because it does not add an extra burden to our Business Office. Your administrator can help your PLC decide how to move forward.
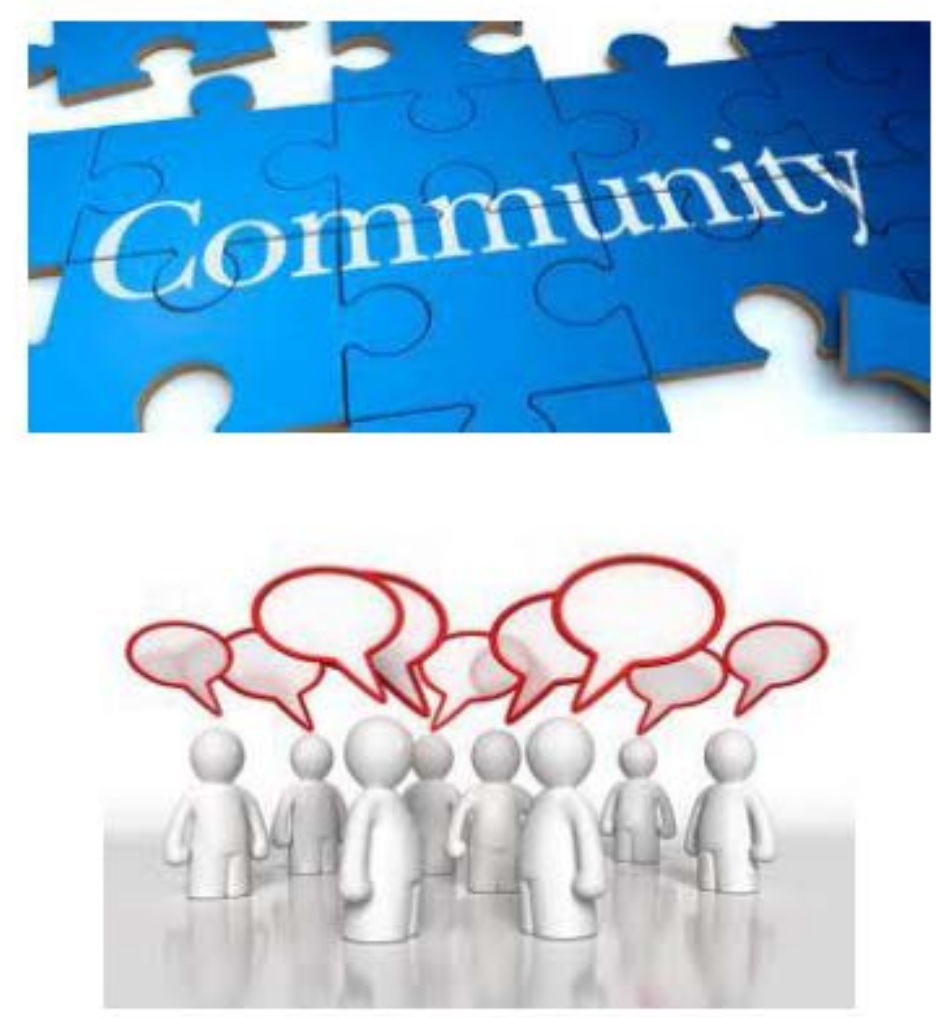


\section{Memorandum of Understanding for a Fiscal Partner Relationship}

\section{-SAMPLE Only-}

This memorandum of Understanding for a Fiscal Partner Relationship is signed between:

(Fiscal Partner's Name) and the Estacada School District.

The fiscal partner agrees to the following responsibilities:

1. Enter into a contractual relationship with the Estacada School District

2. Work with the Estacada School District

3. Report receipt and use of grant funds on state and federal tax forms

The Estacada School District and to the following responsibilities:

(Fiscal Partner's Name) agrees

1. Make monthly progress reports to Estacada School District administration about the status of the project.

2. Turn in cash donations and other cash (from fundraisers) to (Fiscal Partner's Name)

3. Submit requests for checks to the (Fiscal Partner's Name) treasurer, at least three days prior to needing the signed check.

4. Submit receipts for all purchases to the Name) treasurer, attached to each monthly progress report.

5. Send thank you notes to all cash and in-kind donors no less than one week after receipt of a gift.

6. Prepare the final report (except for the bookkeeping portion) of the grant contract.

7. Attend a Board meeting of end of the project and make a final presentation.

Signed on this date:

By Representatives from: (Fiscal Partner's Name) (Signature) (Name, Title)

(Estacada School District Project Rep) (Name, Title) (Estacada School District Administrator) (Name, Title) 


\section{$\underline{\text { ACTIVITY 22.0 }}$}

The district began the 2013-14 school year with a goal of revamping the communication between the district and community. The district would like to share with the community the wonderful things that PLCs are working on. There have been two main communication tools that the district has added to its public relations. These two new methods for communication include: 1) the use of Twitter, and 2) the creation of an electronic district newsletter ("News from the 108 "). These two methods of communication also fall in line with the district's desire to increase the use of instructional technology (noted in Activity 20.0). As a PLC, please complete the activity below to help the district spread the good news regarding what PLCs are accomplishing.
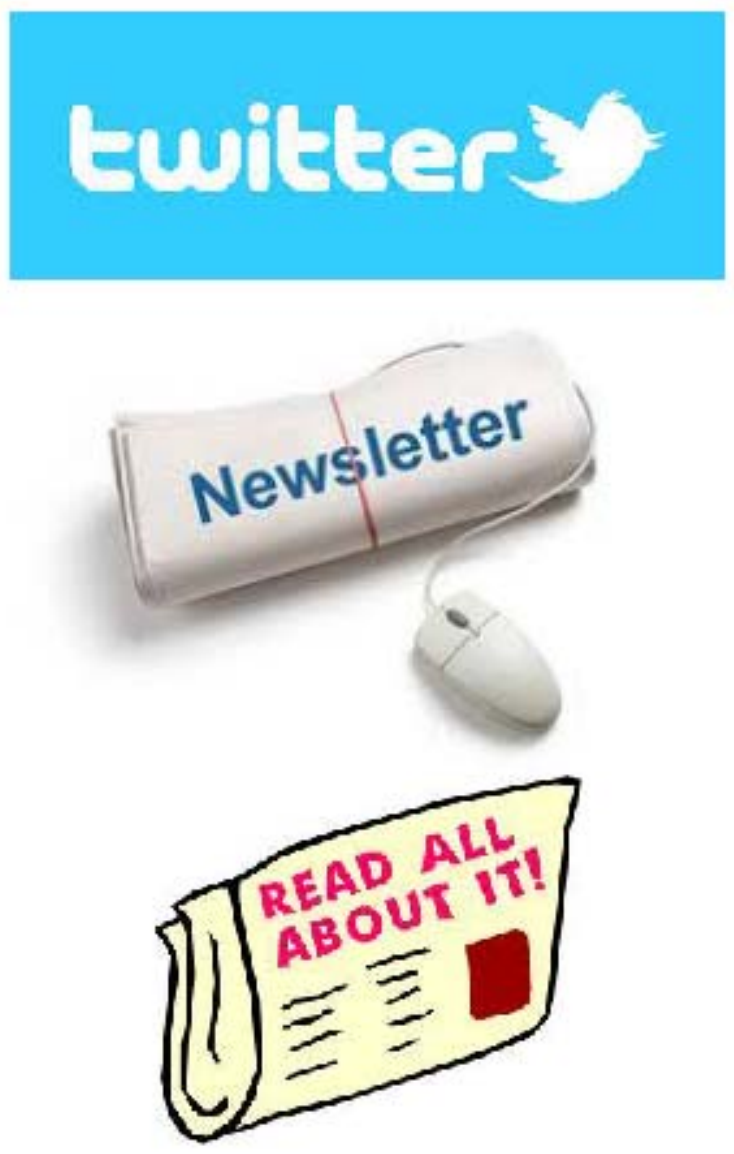


\section{Communication between the District and the Community}

Your assignment, should you choose to accept it, is to be part of a social experiment. As we look to increase communication and infuse technology more and more into our everyday lives (and our instruction with students), it is important to not only be aware of the most current instructional technology methods, but to use them in order to be informed of their potential benefits.

Part 1: You already know more than you think! Please check the instructional technology you have used in the past and/or currently use.

Accelerated Reader

DIBEIS Net

Power School

Talent Ed

Lexia

Moby Math

SWIS

Class Dojo

Promethean Board activities

Learning.com

Mavis Beacon

Study Island

Rosetta Stone

Dreambox

Internet (for research, educational games, etc.)

Khan Academy videos/activities

Others (please list): 
Part 2: Twitter has become a way for schools and educators to "get the word out" to their students and parents. The appeal is that messages are ${ }^{\alpha}$ short and sweet ${ }^{p}$ and can contain pictures, links to websites, and/or attached documents. It can also be used as a way to collaborate with peers and/or facilitate interaction with your class. One of the main barriers to its use is fear that it will be used irresponsibly. Instead of letting fear take over, this could be a perfect opportunity to teach responsibility.

*Before we get started, know that you can deactivate your account at anytime (if you are uncomfortable).

Step 1 - Go to twitter.com (or install and use the Twitter app on your iPad or Smart Phone)

Step 2 - New to Twitter? Sign up

Step 3 - Determine what your "handle" should be

Step 4 - Use the search option (looks like a little magnifying glass in top right corner) to find the following Twitter feed:

@esd108

Step 5 - Click on the button that indicates you would like to ${ }^{\alpha}$ Follow ${ }^{p}$ this Twitter feed.

Step 6 - Scroll through your " Home" page, and answer the question below:

In your opinion, what are @esd108's top three Tweets and why?

1.

2.

3.

In your opinion, what are the top three pictures, web links, or documents included in @esd108's Tweets and why?

1. 
2.

3.

Step 7 - Think about how you might use this platform, and answer the following questions:

If you wanted @esd108 to Tweet one school-related item for you (think pictures, event info, updates, info on cool lessons, fundraising info, field trips, etc.) so that district followers would see it, what would it be?

1.

One way I could possibly use Twitter in my classroom, would be:

1.

\section{EXTRA CREDIT:}

During conferences, get parents and students to create Twitter accounts and have them follow @esd108 so that they can be "in the know" about district events. Make your classroom computers and iPads available and/or be available to show them how to sign up on their Smart Phone.

The staff members who get the most people to sign up will earn a prize!

\section{Homework:}

Discover what the \# (hashtag) means in the world of Twitter and be prepared to discuss at a future staff meeting.

\section{Arecommendation from Administration..}

The Twitter account you just created should be your "professional" Twitter account. You may want to ${ }^{\alpha}$ follow" other Estacada schools/programs/departments, as well as educational journals/agencies (like Ed Week, Edutopia, Scholastic, National Geographic, etc.)

As you become proficient at using Twitter, you may want to create a "personal" account (that you would not necessarily share with students, parents, etc.). Having a "personal" account would allow you to follow your favorite entertainers, sports figures, politicians, etc without worrying about "school people" seeing who you follow. 
Step 8 - As many of you know, the district has created a monthly electronic newsletter called "News from the $108^{n}$ that began its circulation in September 2013. You can help us spread the good word by contributing articles to our newsletter.

Past issues of "News from the $108^{n}$ can be found in the "News and Events" section on the front page of the district's website - www.esdio8.org

Reviewing past articles should give you a sense of what the district is looking for. PLCs around the district are all doing amazing things with students that should not go unnoticed! Please forward the following to your administrator:

- Articles on things your PLC has accomplished with students

- Pictures related to things your PLC has accomplished with students

- Information that the community needs to know about regarding upcoming events, fundraisers, projects, etc.

- Anything else you think our community would benefit from learning about
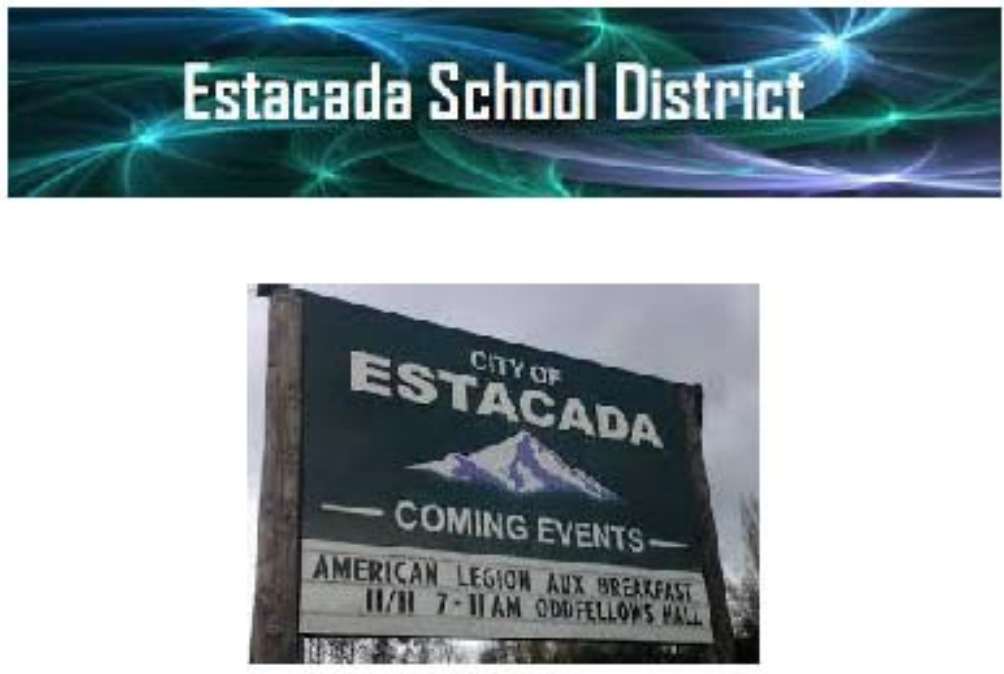


\section{Final Thoughts...}

This handbook is an evolving document. As district and community priorities change, the content of the handbook will change. The activities in the handbook were intended to reflect the current reality and needs of our district and community. We hope that you now have a better understanding of the initiatives being addressed by the district that are either required or highly recommended. Having a systematic approach to the PLC process is the best chance we have (as a district) of adequately addressing these important initiatives.

We also hope you are now aware of initiatives that gained prominence due to increased family and community involvement in Estacada. For families and community members, we hope you can see that the PLC process is crucial to the way we operate as a district, and that there are ways you can get involved. We welcome anyone who is interested in contributing to the creation of an improved learning environment for our students. Please continue to share your ideas so that we can shape our district's future together. 